\title{
Functional and structural brain markers of Alzheimer's disease : clinical studies using EEG and VBM
}

Citation for published version (APA):

van Deursen, J. A. (2009). Functional and structural brain markers of Alzheimer's disease : clinical studies using EEG and VBM. [Doctoral Thesis, Maastricht University]. NeuroPsych Publishers. https://doi.org/10.26481/dis.20090409jd

Document status and date:

Published: 01/01/2009

DOI:

10.26481/dis.20090409jd

Document Version:

Publisher's PDF, also known as Version of record

\section{Please check the document version of this publication:}

- A submitted manuscript is the version of the article upon submission and before peer-review. There can be important differences between the submitted version and the official published version of record.

People interested in the research are advised to contact the author for the final version of the publication, or visit the DOI to the publisher's website.

- The final author version and the galley proof are versions of the publication after peer review.

- The final published version features the final layout of the paper including the volume, issue and page numbers.

Link to publication

\footnotetext{
General rights rights.

- You may freely distribute the URL identifying the publication in the public portal. please follow below link for the End User Agreement:

www.umlib.nl/taverne-license

Take down policy

If you believe that this document breaches copyright please contact us at:

repository@maastrichtuniversity.nl

providing details and we will investigate your claim.
}

Copyright and moral rights for the publications made accessible in the public portal are retained by the authors and/or other copyright owners and it is a condition of accessing publications that users recognise and abide by the legal requirements associated with these

- Users may download and print one copy of any publication from the public portal for the purpose of private study or research.

- You may not further distribute the material or use it for any profit-making activity or commercial gain

If the publication is distributed under the terms of Article $25 \mathrm{fa}$ of the Dutch Copyright Act, indicated by the "Taverne" license above, 


\section{Functional and structural brain markers of Alzheimer's disease: \\ Clinical studies using EEG and VBM}




\title{
๑) J.A. Van Deursen, Maastricht 2009
}

\author{
Cover design | Tess Vuurman \\ Layout | Jacky Rinket \\ Production | Datawyse BV, Maastricht \\ Publisher | NeuroPsych Publishers \\ ISBN | 978-90-75579-39-0
}

\begin{abstract}
NeuroPsych Publishers is a non-profit organisation, which aims at promoting the science of 'Brain and Behavior' and improving the application of the products of this science in health care and education. NeuroPsych Publishers accomplishes these aims by publishing books, dissertations and other products of scientific activity, by disseminating educational materials and publication of tests, assessment scales and other psychometric instruments in the field of Neuropsychology, Neuropsychiatry and other areas within the domain of Brain and Behavior.
\end{abstract}

Postal address: NeuroPsych Publishers

Department of Psychiatry and Neuropsychology

Maastricht University

P.O. Box 616

6200 MD Maastricht 


\title{
Functional and structural brain markers of Alzheimer's disease: Clinical studies using EEG and VBM
}

\author{
Proefschrift \\ Ter verkrijging van de graad van doctor \\ aan de Universiteit Maastricht, \\ op gezag van Rector Magnificus, Prof. mr. G.P.M.F. Mols, \\ volgens het besluit van het College van Decanen, \\ in het openbaar te verdedigen op donderdag 9 april 2009 om 12:00 uur
}

door

Jeroen Antonius van Deursen

Geboren op 17 december 1979 te Helmond 


\section{Promotor}

Prof. dr. W.J. Riedel

Prof. dr. F.R.J. Verhey

\section{Copromotor}

Dr. E.F.P.M. Vuurman

\section{Beoordelingscommissie}

Prof. dr. W.H. Mess (voorzitter)

Dr. E. Formisano

Dr. F.T. Smulders

Prof. dr. C.J. Stam, Vrije Universiteit Amsterdam

Prof. dr. R. Vandenberghe, Katholieke Universiteit Leuven

The research described in this thesis was performed at the Department of Psychiatry and Neuropsychology and the Department of Psychopharmacology, School for Mental Health and Neuroscience, Maastricht University, The Netherlands.

Financial support for the publication of this thesis was kindly provided by Janssen-Cilag, Lundbeck, Novartis, Nutricia, Wyeth Pharmaceuticals, Alzheimer Nederland, Internationale Stichting Alzheimer Onderzoek Nederland (ISAO), AstraZeneca and Danone Research - Centre for Specialised Nutrition. 
The studies in this thesis are based on data collected in two studies. For the EEG and cognition studies presented in chapter 3 through 6, data were collected from 15 patients with Alzheimer's disease, 20 subjects fulfilling the criteria of Mild Cognitive Impairment and 20 healthy control subjects. For the VBM studies described in chapters 7 and 8, data were collected from 37 patients with Alzheimer's disease. Eight AD patients participated in both the EEG and VBM studies. 


\section{Contents}

1 General Introduction 11

$\begin{array}{ll}\text { Part } 1 & 19\end{array}$

2 EEG and other biological markers in Alzheimer's disease:

A review

3 Increased EEG gamma band activity in Alzheimer's disease and mild cognitive Impairment

$4 \quad 40-\mathrm{Hz}$ steady state response in Alzheimer's disease and mild cognitive impairment

5 Response speed, contingent negative variation and P300 in Alzheimer's disease and $\mathrm{MCl}$

6 Comparison of EEG parameters in the diagnosis of Alzheimer's disease and $\mathrm{MCl}$

Part 2

7 Neuroanatomical correlates of neuropsychiatric syndromes in Alzheimer's disease

8 Neuroanatomical correlates of apathy in Alzheimer's disease

9 General Discussion

Summary 175

Samenvatting 181

References

Dankwoord

Curriculum Vitae

Publications 





\section{General Introduction}

\subsection{Alzheimer's disease and mild cognitive impairment}

Alzheimer's disease $(A D)$ is the most common neurodegenerative condition of unknown aetiology. Currently 25 million patients suffer from $A D$ and this number is estimated to rise to 114 million patients worldwide by 2050 (Wimo et al., 2003). At microscopic level, AD is characterized by the presence of large numbers of neuritic plaques, neurofibrillary tangles and by neuronal cell loss beginning in the hippocampus. Clinically $A D$ manifests itself by progressive decline of memory and other cognitive functions. The progressive course of memory decline in AD is accompanied by neuropsychiatric symptoms, which complicate the activities of daily life and the care for these patients. Neuropsychiatric symptoms include mood changes, apathy, irritability, anxiety, delusions, hallucinations, agitation, sleep disturbances and changes in appetite. Mild cognitive impairment $(\mathrm{MCl})$ is often considered to be the transitional stage between normal aging and dementia, but only $10-15 \%$ of the $\mathrm{MCl}$ patients progress to Alzheimer's disease (Petersen et al., 1999). To date there are only symptomatic treatments for Alzheimer's disease. Most Alzheimer patients are treated with cholinesterase inhibiting drugs. Although these drugs improve cognition, and daily life functioning, and they slow down the progression of the disease (Bullock and Dengiz, 2005; Cummings, 2003; Doody et al., 2001), the fundamental causes of Alzheimer's disease remain unaffected by these drugs. In the search for a causal treatment of $A D$ there is an urgent need for objective measures that provide more insight into the cognitive, neuropathological, morphological and neurophysiological changes in AD. Such objective measures, usually called biomarkers, should contribute to its diagnosis, early detection and to the follow-up of therapeutic interventions in cases of $\mathrm{AD}$ and $\mathrm{MCl}$. 
In the present thesis, the roles of behavioral and electrophysiological paradigms as candidate biomarkers in $A D$ and $\mathrm{MCl}$ are assessed. This thesis further investigates the neuro-anatomical correlates of neuropsychiatric disorders.

\subsection{Biological markers}

The terms biomarker (biological marker), clinical marker and surrogate endpoint are not consistently used in medical literature. Therefore the definitions of these terms are presented here. These definitions were cited from (De Gruttola et al., 2001).

Biological Marker (Biomarker):

A characteristic that is objectively measured and evaluated as an indicator of normal biologic processes, pathogenic processes or pharmacological response to a therapeutic intervention.

Clinical Endpoint:

A characteristic or variable that reflects how a patient feels or functions, or how long a patient survives.

Surrogate Endpoint:

A biomarker intended to substitute for a clinical endpoint. A clinical investigator uses epidemiologic, therapeutic, pathophysiologic, or other scientific evidence to select a surrogate endpoint that is expected to predict clinical benefit, harm or lack of benefit or harm.

In order to determine whether a candidate biomarker is a valid marker, certain criteria have to be met. The Ronald and Nancy Reagan Research Institute of the Alzheimer's Association, has formulated guidelines for these criteria. According to these, a candidate biomarker should include as many of the following features as possible:

1. Should be able to detect a fundamental feature of Alzheimer's neuropathology

2. Should be validated in neuropathologically confirmed AD cases

3. Should be precise (able to detect $A D$ early in its course and distinguish it from other dementias)

4. Should be reliable

5. Should be non-invasive

6. Should be simple to perform

7. Should be inexpensive 
Furthermore the sensitivity should be higher than $80 \%$ and the differentiation between other forms of dementia should exceed $80 \%$ (1998). The neurophysiological and behavioral biomarkers investigated in the current thesis will be evaluated on the basis of these criteria.

\subsection{EEG and structural brain imaging}

Electroencephalography (EEG) is a method that is used to study electrical brain activity. In general the EEG is studied in the frequency domain and in the time domain (i.e. with event related potentials). The classical EEG frequency spectra are the delta band (1-4 Hz), theta band (4-8 Hz), alpha band (8-13 Hz) and the beta band $(13-30 \mathrm{~Hz})$. In recent years there has been a growing interest in the high EEG frequencies (i.e. the gamma band) as it seems to be important in cognitive and perceptual processes (Singer and Gray, 1995). Event-related potentials (ERPs) provide information about neural processes involved in different types of stimuli. An advantage of EEG measurements is that they directly measure neural activity.

The second method that was used in the present thesis involves structural MRI using Voxel-based Morphometry (VBM) as an analytical instrument. VBM is a method that is used to assess atrophy of the gray matter (GM) over the whole brain. This method further makes it possible to identify local brain atrophy associated with specific behavioral aspects. The VBM method is especially suitable when there is no a-priori assumption specifying the brain region of interest (Ashburner and Friston, 2000).

\subsection{Aims of the thesis}

The aims of the present thesis are: to evaluate the electrophysiological and structural changes of the brain as candidate biomarkers in Alzheimer's disease and $\mathrm{MCl}$; to relate these changes to cognitive performance and behavior; and to determine their sensitivity, specificity and reliability.

The first objective of this thesis is to gain more insight into the value of electrophysiology as a biomarker in the diagnosis and follow-up of $\mathrm{AD}$ and $\mathrm{MCl}$. In order to achieve this objective the sensitivity, specificity and reliability of a number of EEG paradigms have been examined in AD and $\mathrm{MCl}$ patients and in a healthy control group. The main research questions for the first objective of this thesis are: 
Functional and structural brain markers of Alzheimer's disease | Chapter 1

1. Can EEG paradigms reliably discriminate patients with $A D$ from $\mathrm{MCl}$ and healthy control subjects?

2. Which EEG paradigm is the most sensitive, specific and reliable when it comes to discriminating $A D$ patients from $\mathrm{MCl}$ and healthy control subjects?

3. Does EEG have an additional value in the diagnosis and the follow-up of $A D$ ?

The second objective of this thesis was to examine the neural correlate of behavioral disorders in AD. Voxel based morphometry was used to study volumetric changes in the brain that are associated with behavioral disorders. The research question for the second objective was:

4. Which cortical brain areas are involved in the pathogenesis of neuropsychiatric disorders in $A D$ ?

\subsection{Outline of the thesis}

The present thesis is organized as follows. The first part of the thesis (chapters 2 to 6) discusses the value of EEG as a candidate biomarker in $A D$ and $\mathrm{MCl}$. Chapters 3 to 6 investigate the diagnostic sensitivity and reliability of the following EEG measures; induced gamma-band oscillations, 40-Hz steady-state response (SSR), contingent negative variation (CNV), and a classical oddball paradigm. The second part of this thesis (chapters 7 and 8) discusses the neural correlates of behavioral disorders in AD.

In chapter 2 an overview is given of different biomarkers that are used in AD and $\mathrm{MCl}$ research. These biomarkers are compared with EEG parameters and evaluated with regard to their usefulness as a diagnostic marker, as a marker for early detection, and as a marker of therapeutic change. The aim of this overview is to assess the additional value of EEG in comparison with other biomarkers with regard to $A D$ and $\mathrm{MCl}$.

Chapter 3 examines the diagnostic sensitivity and reliability of EEG induced gamma band oscillations in $\mathrm{AD}$ and $\mathrm{MCl}$. These high-frequency $(30-100 \mathrm{~Hz}$ ) oscillations are considered to be important for primary sensory processing and higher cognitive functions (Kaiser and Lutzenberger, 2003; Kaiser and Lutzenberger, 2005). Passive tasks such as music listening, story listening and movie 
watching previously showed augmentation of the gamma band. The aim of this chapter is to study whether gamma band oscillations induced by these passive tasks could discriminate between $A D, M C l$ and control subjects. Fifteen unmedicated $A D$ patients, $20 \mathrm{MCl}$ patients and twenty healthy control subjects performed a music listening, a story listening and a visual stimulation task in a test-retest design while EEG was recorded.

It was hypothesized that gamma band oscillations would discriminate between $A D$ patients, $\mathrm{MCl}$ patients and controls and that the control group would show the highest gamma band power and the $A D$ group the lowest.

Chapter 4 assesses the $40-\mathrm{Hz}$ steady-state response (SSR) as a biomarker with regard to $A D$ and $\mathrm{MCl}$. The $40-\mathrm{Hz}$ SSR reflects sensory gating and early sensory processing (Joliot et al., 1994). Previous studies showed decreased $40-\mathrm{Hz}$ SSR in subjects with schizophrenia and increased $40-\mathrm{Hz}$ SSR in subjects with AD. The aim of this chapter was to determine whether the 40-Hz SSR paradigm could discriminate between $A D, M C l$ and healthy controls. Fifteen $A D$ patients, 20 $\mathrm{MCl}$ patients and 20 healthy control subjects were stimulated with auditory click trains of $40-\mathrm{Hz}$ in a test-retest design while EEG was recorded.

It was hypothesized that the 40-Hz SSR would be higher in the AD group compared to the $\mathrm{MCl}$ and control group. It was furthermore expected that this paradigm is sensitive and reliable enough to discriminate between the three groups.

Chapter 5 discusses the relation of a classical oddball paradigm and a contingent negative variation (CNV) paradigm with response speed in $A D$ and $\mathrm{MCl}$. Recent studies have shown that response speed measures are very sensitive indicators in longitudinal follow-up studies. The combined measurement of response speed and the associated ERP's can provide more insight into the neural fundaments of slowed response speed in $A D$ and $\mathrm{MCI}$. If response speed and the associated ERP's are to be used as biomarkers, their mutual relation needs to be clarified. The present chapter further aims to assess whether there is an association between response speed and the ERP's evoked by the CNV and the oddball paradigm. A further aim is to examine whether these measures can discriminate between subjects with $A D, M C l$ and healthy controls. $C N V$ is used as an electrophysiological correlate of anticipatory attention and motor readiness. The oddball paradigm is used to assess signal processing and stimulus characterization. Fifteen AD patients, $20 \mathrm{MCl}$ patients and 20 control subjects were studied in a test-retest design. 
Functional and structural brain markers of Alzheimer's disease | Chapter 1

It was hypothesized that the amplitude of the CNV is decreased in subjects with $A D$ compared to subjects with $\mathrm{MCl}$ and healthy controls and that this correlates with decreased response speed. Furthermore, it was expected that the P300 amplitude is lower and the latency is higher in the $A D$ group compared to the $\mathrm{MCl}$ group and control group.

In chapter 6 the previously discussed EEG paradigms are compared with each other to examine which paradigms are the most accurate and reliable in distinguishing $A D$ from $\mathrm{MCl}$ and healthy controls. Most of the previously discussed EEG paradigms showed differences between the patient groups. To determine which of these paradigms have the most potential as a candidate biomarker, the sensitivity, specificity and reliability of these paradigms are compared head to head. The criteria for candidate biomarkers presented earlier in the introduction, are applied to the investigated electrophysiological and behavioral measures.

It was hypothesized that the oddball paradigm and gamma band changes would be most sensitive, specific and reliable in discriminating $A D$ from $\mathrm{MCl}$ and healthy control subjects.

Chapter 7 focuses on volumetric changes in the gray matter which are associated with neuropsychiatric syndromes. Neuropsychiatric disorders are a common feature of $A D$ and the neurobiological causes are not fully understood. At present, neuroimaging studies have focused on the association between separate individual symptoms and changes in brain structure and function. The identification of neuropsychiatric syndromes suggests a common pathogenesis. In order to determine which brain areas are associated with the presence and severity of neuropsychiatric syndromes, voxel based morphometry (VBM) is used. VBM is a method used to assess atrophy of the whole brain in an automated manner.

It was hypothesized that there is a significant association between the presence and severity of neuropsychiatric syndromes and gray matter atrophy.

Chapter 8 discusses the neural correlates of apathy in AD. Apathy is one of the most common neuropsychiatric disorders in AD. Apathy is considered to consist of several sub-domains, such as emotional blunting, lack of interest and lack of initiative. At present, most neuroimaging studies focused on apathy in general 
and paid little attention to the sub-domains. As a result, the neuroanatomical correlates of the apathy sub-domains remain unclear. This chapter aims to study the neuronatomical correlates of the sub-domains of apathy using VBM.

It was hypothesized that there is a significant correlation between the apathy sub-domains and local gray matter atrophy in the frontal and anterior cingulate cortex.

Chapter 9 summarizes the main findings of this thesis and discusses the usefulness of the present study for future research. 

Part 1 



$$
\text { Chapter }
$$





\title{
EEG and other biological markers in Alzheimer's disease: A review
}

J.A. van Deursen, E.F.P.M Vuurman, F.R.J. Verhey, W.J. Riedel.

\begin{abstract}
In this study the available literature focusing on biological markers for the diagnosis and follow-up of AD is reviewed. The aim was to compare EEG with other biological markers in order to determine the value of EEG measures as candidate biomarkers in AD. The value of EEG measures is compared to cognitive markers, biochemical markers and markers derived from anatomical- and functional neuroimaging data. Moreover, the reliability, sensitivity and specificity of these markers were assessed. The conclusion of this review was that EEG measures could yield valuable candidate marker for the follow-up and evaluation of therapeutic interventions in AD. The review further discussed that with regard to new EEG paradigms there is little knowledge on their sensitivity, specificity and reliability.
\end{abstract}




\subsection{Introduction}

Alzheimer's disease $(A D)$ is a neurodegenerative condition of unknown etiology. At microscopic level, it is characterized by the presence of large numbers of neuritic plaques, neurofibrillary tangles and by neurodegeneration beginning in the hippocampus. $A D$ is the most common form of dementia, affecting 25 million patients worldwide (Wimo et al., 2003). Age is the most important risk factor for the development of AD. With an increasing life expectancy worldwide, the number of elderly at risk of developing $A D$ is increasing. The large number of patients suffering from this devastating disease, now and in the future, urges the need for good medical treatment.

To date only symptomatic treatments for Alzheimer's disease are available. Most Alzheimer patients are treated with cholinesterase inhibiting drugs. Although these drugs improve cognition and daily life activity, and slow down the progression of the disease (Bullock and Dengiz, 2005; Cummings, 2003; Doody et al., 2001), the fundamental causes of Alzheimer's disease remain unaffected by these drugs.

Researchers, clinicians and the pharmaceutical industry have shown a growing interest in the development of causal treatment interventions or better symptomatic treatments. An important aspect of the development of new therapies is the development and evaluation of good (bio) markers. These markers are important for the early detection of $A D$, and ultimately for the evaluation of therapeutic effects. Biomarkers are the endpoints of biological changes that emerge during the development and progression of a disease. In contrast, surrogate markers are used in clinical trials as an alternative for traditional endpoints. Surrogate markers are variables, which are included in clinical trials when it is inconvenient to measure the variable of primary interest.

Due to technological developments in recent years, the development of new biomarkers has led to a better understanding of the healthy and diseased brain. Of these biomarkers, the electroencephalogram (EEG) is one of the most direct ways to measure changes in brain activity. However, in the field of research focused on Alzheimer's disease, EEG is not often used as a diagnostic marker, nor as a surrogate marker in clinical trials. Recent developments in analytical and computational techniques have created a number of new opportunities for the use of EEG imaging to study Alzheimer's disease.

This review aims to evaluate the value of EEG as a candidate marker in AD. To examine its value as a new marker it will be evaluated with regard to three aspects. First of all, the value of EEG as a diagnostic marker will be examined. Secondly, its value as a marker for early detection and prediction will be deter- 
mined. Thirdly, its value as an endpoint marker for therapeutic changes will be evaluated.

In the following review, the value of EEG will be compared to cognitive markers, biochemical markers and markers from anatomical and functional neuroimaging. Moreover, the content validity, sensitivity and specificity of these markers will be evaluated.

\subsection{Criteria for Alzheimer's disease}

\subsubsection{Clinical criteria for Alzheimer's disease}

The clinical criteria for Alzheimer's disease as described by the NINCDS-ADRDA criteria (McKhann et al., 1984) serve as the international guideline for the diagnosis of Alzheimer's disease. These criteria are in perfect harmony with the criteria outlined in the DSM-IV (American Psychiatric Association, 1994). According to these criteria, the diagnosis of dementia is marked when memory impairment and one or more other cognitive deficits occur, which in turn impair social and occupational functions.

The clinical diagnosis of dementia describes the psychiatric syndrome without indicating a fundamental cause. The diagnosis is based on behavior and cognition and can at present not be determined by laboratory or neuroimaging findings alone (McKhann et al., 1984). The clinical diagnosis of Alzheimer's disease is also based on the exclusion of other causes of dementia and takes the typically slow progressive course of the disease over time into consideration (McKhann et al., 1984). Laboratory and neuroimaging procedures are used to determine the causes of the dementia and contribute to its differential diagnosis.

\subsubsection{Neuropathological criteria for Alzheimer's disease}

The Consortium to Establish a Registry for Alzheimer's Disease (CERAD) has established a protocol that reflects an international consensus on how specific brain areas should be analyzed in order to examine them for Alzheimer's neuropathology. The CERAD protocol indicates that microscopic analysis is required of the middle temporal gyri, inferior parietal lobe, hippocampus, entorhinal cortex, and the midbrain including the substantia nigra. This protocol assesses the severity of the neuropathological features by using a semi-quantitative analysis.

The CERAD criteria do not account for neurofibrillary tangles. A consensus report by 'The National Institute on Aging, Reagan Working Group on Diagnostic Criteria for the Neuropathological Assessment of Alzheimer's Disease' does 
address this problem and recommends that neurofibrillary tangles should be taken into account for the neuropathological diagnosis of Alzheimer's disease.

A consensus exists that the diagnosis is most accurate when the CERAD criteria are used together with the Braak staging criteria (Braak and Braak, 1991) for neurofibrillary tangles.

\subsubsection{Discrepancy between clinical and neuropathological criteria}

In the search for new biomarkers for a disease, the quality of the gold standard used is of pivotal importance. In the case of Alzheimer's disease, the determination of a gold standard is a problematic issue.

The consensus exists that the post-mortem confirmation of the clinical diagnosis is the only way to be $100 \%$ accurate. On the basis of this evidence, a discrepancy has been revealed between the NINCDS-ADRDA and CERAD criteria, as the clinical criteria do not seem to predict the presence of neuropathological lesions with complete accuracy. A recent study that compared these criteria showed that the NINCDS-ADRDA criteria are $86 \%$ sensitive for neuropathologic changes (Hogervorst et al., 2003). A meta-analysis of 13 studies showed that the average sensitivity of NINCDS-ADRDA and DSM-IIIR for AD neuropathology was $81 \%$ (range $49 \%-100 \%$ ), and that the specificity was 70\% (range 47\% - 100\%) (Knopman et al., 2001). Another study, which compared the post-mortem findings of 209 individuals with their ante-mortem cognitive performance, showed that $30 \%$ of the patients diagnosed with AD post-mortem had not been diagnosed as demented ante-mortem (Neuropathology Group of the Medical Research Council Cognitive Function and Ageing Study (Neuropathology Group of the Medical Research Council Cognitive Function and Ageing Study (MRC CFAS), 2001). The latter study furthermore addressed the importance of small vascular lesions, which were more common in $A D$ patients than in non-demented subjects.

These studies indicate that there is a discrepancy of about $30 \%$ between the clinical criteria and post-mortem findings. This discrepancy makes it difficult to validate new biomarkers and raises the question which criteria should be used as the gold standard when searching for new biomarkers.

\subsubsection{The gold standard}

In studies focused on the search for new biomarkers, a post-mortem confirmation of the AD diagnosis should serve as the gold standard. However, this is nearly impossible for practical reasons such as: a long lapse between studies, small study samples and ethical considerations. Therefore, the clinical NINCDS-ADRDA and DSM-IV criteria supported by laboratory and neuroimaging findings should be considered as the gold standard in AD diagnosis. 
This raises the following question: which criteria should new (bio) markers meet? The Ronald and Nancy Reagan Research Institute has formulated guidelines for these criteria. According to these guidelines, a valid biomarker should detect manifestations that are fundamental to the neuropathology in $A D$. The sensitivity should be higher than $80 \%$ and the differentiation between other forms of dementia should exceed $80 \%$. The biomarker should also exhibit high test-retest reliability, be non-invasive and easy to perform. Furthermore, a biomarker should be a good predictor of the progression of the disease and should have a high correlation with (future) cognitive performance (The Ronald and Nancy Reagan Research Institute of the Alzheimer's Association and the National Institute on Aging Working Group, 1998).

\subsection{Cognitive Markers}

Clinically, $A D$ is characterized by: impaired memory, impaired language functions, apraxia, agnosia, and loss of temporal and spatial orientation and the presence of behavioral disorders. The cognitive hallmarks of AD are discussed in this section.

\subsubsection{Episodic memory}

The most profound and early symptom of Alzheimer's is the deficit in episodic memory. Episodic memory is known to gradually become impaired during the years prior to the manifestation of Alzheimer's disease (Backman et al., 2004). The evaluation of memory reveals that in cases of patients with $A D$, recall and recollection are impaired in both the verbal and non-verbal domains (Storandt and Hill, 1989).

Memory tests have also proved to be instruments with a high sensitivity and a high specificity in discriminating $A D$ from $\mathrm{MCl}$ and vascular dementia (De Jager et al., 2003). The examination of delayed recall and recognition is a better way to assess the consolidation phase of memory than immediate recall and recognition (Backman et al., 2004). Hence, impaired delayed recall has been identified as a specific cognitive marker that discriminates $A D$ from other neurodegenerative disorders, such as Korsakoff's syndrome and Huntington's disease (Moss et al., 1986).

In distinguishing mild AD patients from healthy elderly subjects, the use of delayed recall is a very sensitive tool while the use of immediate recall and recognition performance yields poor results (Welsh et al., 1991; Welsh et al., 1992). In contrast, when diagnosis is not the aim and memory functioning is 
used to identify the stage of $A D$, the use of delayed recall yields poor results. Other cognitive measures, i.e. fluency, praxis and recognition memory revealed properties better suited to the staging of the disease (Welsh et al., 1992).

These data suggest that impaired episodic memory measured by delayed recall is a very sensitive marker for early AD. Memory items are actually the only group of items not scored according to a subjective assessment by the evaluating clinician, which is particularly the case when language functions are being evaluated. Memory items comprise 25 out of the total 70 points (39\%) of the Alzheimer's Disease Assessment Scale (ADAS-cog), whereas language makes up $36 \%$, praxis $14 \%$ and orientation $11 \%$ (Rosen et al., 1984; Verhey et al., 2004). The ADAS-cog serves as the gold standard in the evaluation of therapeutic interventions in AD. The decline in episodic memory has been shown to be the most robust cognitive marker of (early) Alzheimer's disease.

\subsubsection{Executive functions}

Executive functions (i.e. goal attainment, planning, and execution) are impaired by Alzheimer's disease (Perry and Hodges, 1999). A retrospective study has shown that executive functions are a sensitive tool for differentiating between early $A D$ patients and patients who remain non-demented (Chen et al., 2001). However, this study also showed that a decline in executive functions is not very specific when it comes to distinguishing Alzheimer's disease from other neurodegenerative dementias. It also indicated that sustained attention remains fairly intact in patients with mild $A D$ impairment as the disease progresses. Hence, although the impairment of attention is often reported in Alzheimer's disease, this does not seem to indicate an AD-specific decline, since it is also present in other neurodegenerative disorders (Duke and Kaszniak, 2000).

\subsubsection{Speed of processing}

Speed of processing is classically assessed by simple (SRT) and choice reaction time tasks (CRT).

Impaired speed of processing is a characteristic of Alzheimer's disease and distinguishes patients from elderly controls (Gordon and Carson, 1990; Levinoff et al., 2005; Storandt and Beaudreau, 2004). Decreased speed of information processing was also shown to be present in patients with $\mathrm{MCl}$ (Levinoff et al., 2005). The specificity of processing speed is low since it is also present in dementias, which have their origin in a vascular or sub-cortical cause (Mendez et al., 1997).

Despite its low sensitivity, several studies have proved that measures of speed of information processing are very sensitive in the detection of the effect 
of treatments on patients with AD (Wesnes, 2008). A 12-week follow-up study that assessed the effects of galantamine on cognitive performance in patients with $A D$ showed that speed measures were more sensitive indicators of changes than the ADAS-cog (Caramelli et al., 2004). These studies suggest that speed of information processing might be very interesting as a marker in therapeutic interventions.

\subsubsection{Considerations}

An unfavourable aspect connected to the assessment of cognitive performance is that it is often subject to day-to-day fluctuations and motivational aspects. Furthermore, AD patients often have difficulty understanding the test instructions and receive variable kinds of support from raters. These aspects can have a major effect on the results of a neuropsychiatric evaluation.

\subsection{Biochemical Markers}

The intracellular accumulation of abnormal tau filaments and the extra cellular deposition of amyloïd- $\beta$ proteins compromise the viability and function of the affected neurons. How these pathological deficits lead to massive neurodegeneration in $A D$, is not fully understood. Nevertheless, several biomarkers in the cerebral spinal fluid (CSF) are directly related to the biological mechanism that leads to the accumulation of these plaques and tangles. These biochemical biomarkers are discussed in this section.

\subsubsection{Beta-amyloïd}

Amyloïd-B(1-42) is the main constituent of neuritic plaques and is a proteolytic product formed by the cleavage of the amyloïd precursor protein (APP). The amyloïd- $\beta$ protein is produced in normal cellular metabolism and secreted in the extra cellular space, the CSF (Haass et al., 1992). In AD, the APP is cleaved by $B$-secretase and $B$-secretase, resulting in the insoluble amyloïd- $B$ protein. This amyloïd- $\beta$ accumulates in the extra cellular matrix and leads to the formation of plaques. The decline of the level of amyloïd- $\beta$ in the CSF is considered to reflect the amyloïd deposition in the brain (Hampel et al., 2004). There is evidence that the level of amyloïd- $B$ in the CSF is inversely correlated to the number of plaques (Strozyk et al., 2003).

A pooled analysis showed that changes in the CSF level of amyloïd- $\beta$ have a high sensitivity (i.e. $90 \%$ ) and specificity (i.e. $86 \%$ ) in discriminating between 
$A D$ and healthy elderly subjects (Blennow and Hampel, 2003). Despite these high levels of sensitivity and specificity, decreased CSF levels of amyloïd- $B$ are also evident in vascular dementia, Lewy body dementia, amyotrophic lateral sclerosis, multi system atrophy and Creutzfeldt-Jacob disease; whereas normal CSF amyloïd- $\beta$ levels were also found in patients suffering from depression, Parkinson's disease and progressive supranuclear palsy (Andreasen and Blennow, 2005).

Several other studies showed that in discriminating AD from other neurodegenerative dementias, the specificity and sensitivity of changes in CSF amyloïd$B$ is poor (Kapaki et al., 2003). This indicates that the additional value of CSF measures of amyloïd- $\beta$ in the diagnosis of $A D$ is questionable.

\subsubsection{Tau}

Tau is a cytoskeletal phosphoprotein, which promotes the binding of tubuline in microtubules and thereby promotes their assembly in stability. The tau protein is known to have six isoforms and numerous phosphorylation sites. Microtubules are essential in the formation of axons and play a crucial role in the axonal transport of proteins necessary for neurotransmission. Abnormal phosphorylation of tau leads to the abnormal intracellular accumulation of tau, leading to impaired axonal transport and ultimately cell death. In the CSF, total tau (t-tau) and phosphorylated tau ( $p$-tau) can be detected by their binding to monoclonal antibodies in immunoassays.

\subsubsection{Total tau}

High CSF levels of tau reflect neuronal and primarily axonal damage. Elevated levels of $\mathrm{t}$-tau increase with age and are considered to reflect neurodegeneration in normal aging (Sjogren et al., 2001). In discriminating AD patients from healthy elderly subjects, a pooled analysis showed that elevated t-tau has a high sensitivity $(90 \%)$ and specificity $(80 \%)$. Depressive disorders, Parkinson's disease, alcoholic dementia, and progressive supranuclear palsy show normal levels of t-tau. However, elevated t-tau levels have also been demonstrated in other neurodegenerative disorders like vascular dementia (Andreasen and Blennow, 2005). Elevated CSF levels of t-tau were also found in subjects after traumatic brain injuries (Zemlan et al., 1999) and acute stroke (Hesse et al., 2001), while p-tau levels remained unchanged. These studies suggest that elevated levels of t-tau in the CSF are more likely the result of general brain damage than of an Alzheimer-specific mechanism. 


\subsubsection{Phosphorylated tau}

CSF levels of phosphorylated tau (p-tau) reflect the abnormal phosphorylation of tau proteins. The concentration of phosphorylated tau in the CSF probably reflects the general state of phosphorylation in the brain. The CSF levels of p-tau are increased in $A D$ and were found to be sensitive (92\%) and specific $(80 \%)$ in discriminating between $A D$ and healthy elderly subjects (Blennow and Hampel, 2003).

A crucial finding is the high specificity of p-tau for Alzheimer's disease. Elevated CSF levels of $p$-tau have not been shown in other neurodegenerative disorders such as vascular dementia, fronto-temporal dementia, Lewy body dementia and depressive disorders (Andreasen et al., 1999; Blennow et al., 1995). In $\mathrm{MCl}$ patients elevated p-tau levels are higher than in healthy elderly subjects, which suggests that changes in p-tau can be detected very early in the course of the disease (Buerger et al., 2002). Another study showed that p-tau levels are elevated early in the course of $A D$, but that it decreases as $A D$ progresses (Hampeletal., 2001). The authorscontributethisfinding to increased sequestration of $\mathrm{p}$-tau into neurofibrillary tangles and to a decrement of solubility of $\mathrm{p}$-tau in the CSF. This finding suggests that $p$-tau can have diagnostic value, but mainly in early AD. Of the CSF markers discussed here, $p$-tau is the marker with the highest sensitivity and specificity for $A D$.

\subsubsection{CSF markers: considerations}

Viewed together, these studies show that CSF levels of amyloïd- $B$, t-tau and $p$-tau show high sensitivities and high specificities in discriminating $A D$ from healthy elderly subjects. However, p-tau is the only marker in the CSF that can have an additional value for the diagnosis of $A D$, since it is the only one that discriminates $A D$ from other types of dementia. When the CSF markers discussed above are used in combination, the sensitivity and specificity increases (Andreasen and Blennow, 2005). A negative aspect of CSF studies is that they reflect the pathological aspect of $A D$, which does not always correlate to the clinical manifestation of symptoms as shown above (Neuropathology Group of the Medical Research Council Cognitive Function and Ageing Study (MRC CFAS), 2001). Another drawback of CSF markers is that the lumbar puncture, which is necessary for CSF collection, is an invasive method. 


\subsection{Neuroimaging Markers}

Cerebral atrophy is one of the major hallmarks of Alzheimer's disease and is the resultant of the neurodegenerative process. In the diagnostic process, two neuroimaging techniques are commonly used to study a patient's brain for anatomical changes due to atrophy. Computational Tomography (CT) is x-ray based and provides images with poor spatial resolution in comparison to the alternative technique viz. Magnetic Resonance Imaging (MRI). Furthermore, the postprocessing possibilities with regard to $\mathrm{CT}$ are exceeded by the possibilities with MRI. For these reasons, CT studies will not be discussed in this section. The first part of this section will discuss structural neuroimaging techniques and the second part will discuss functional MRI neuroimaging techniques.

\subsubsection{Structural neuroimaging}

In clinical practice, structural MRI is used to exclude other aetiologies for dementia in patients with probable AD. Cerebral atrophy and especially hippocampus atrophy are among the most consistent findings in AD (Atiya et al., 2003; Csernansky et al., 2004; Fox and Schott, 2004; Fox et al., 1999; Kantarci and Jack, 2004; Wang et al., 2003). Temporal lobe and hippocampus volume loss appear to be more specific for Alzheimer's disease than cerebral atrophy (Csernansky et al., 2004). In discriminating AD from $\mathrm{MCl}$ and control subjects, hippocampus volume loss seems to be a sensitive and specific measure (Bottino et al., 2002; Goncharova et al., 2001; Gosche et al., 2002). However, reduced hippocampus volume was also found in a large number of other neurodegenerative disorders (Geuze et al., 2005), which suggests that it is not a characteristic that is very specific for $A D$.

A few studies have examined the correlation between hippocampus volume loss and post-mortem neuropathological findings. Csernansky and colleagues (2004) discovered a strong relation between the density of neurofibrillary tangles in the hippocampus and its ante-mortem volume. Results from the NUN-Study show a very strong correlation between post-mortem neuropathology and post-mortem MRI hippocampus volume assessment (Gosche et al., 2002). Another critical finding was the inverse correlation between the Clinical Dementia Rating scale (CDR) (Morris, 1993), and the hippocampus volume (Csernansky et al., 2004). These data suggest a triangular correlation between tangle density, severity of dementia and hippocampus volume.

The studies discussed here all used manual selection of the regions of interest (ROI), in most cases the hippocampus or the medial temporal lobe. Disadvantages of these manual and ROI based analyses are: inter-observer discrepancy, the 
manual selection of ROI boundaries and the extremely long time required for the analysis (Pruessner et al., 2000). Some of these disadvantages can be avoided by computational analysis techniques such as voxel-based morphometry.

\subsubsection{Voxel-Based Morphometry}

Voxel Based Morphometry (VBM) is a method to calculate volume changes of brain areas in whole-brain analysis. This computational method allows researchers to examine volume changes of the gray matter (GM), white matter (WM) and CSF separately. A consistent finding in $A D$ is the loss of $G M$ volume in the hippocampus and the parahippocampal cortex (Busatto et al., 2003; Chetelat et al., 2005; Hirata et al., 2005; Karas et al., 2003; Karas et al., 2004). Furthermore, GM loss was also reported in the entorhinal cortex (Busatto et al., 2003), medial thalamus and caudate nucleus (Karas et al., 2003).

In longitudinal follow-up studies of $\mathrm{MCl}$ patients there is a significantly faster rate of $\mathrm{GM}$ volume loss in the $\mathrm{MCl}$ converters compared to non-converters in the hippocampus, parahippocampal cortex, medial temporal lobe, and parietal lobe (Chetelat et al., 2005; Karas et al., 2008a; Visser et al., 2002). The $\mathrm{MCl}$ converters also showed smaller $\mathrm{GM}$ volumes at baseline. The findings from VBM analysis of GM are in accordance with findings in studies that used a manual ROI approach.

VBM has proved to be a very elegant way to measure atrophy in crosssectional studies as well as in longitudinal studies. The automated computational procedures in VBM are not affected by inter-rater differences and anatomical boundary shifts are minimized by the utilization of statistical maps and segmentation techniques (Barnes et al., 2004). Another advantage of VBM is that the whole brain is analyzed at once. It does not require an a-priori hypothesis on specific brain regions like the ROI approach. A major weakness in the pre-processing is the normalisation step where the scan is warped to a standard template. Especially when degenerated brains are studied, this step might induce errors in the data set. This possible bias should be taken into account when using this method. VBM is especially an excellent method when the hypotheses of a study do not involve specific brain areas. When a specific brain area is to be analysed, the ROI-based approach is probably more accurate than the VBM method.

\subsubsection{PET ligand imaging}

Developments in molecular imaging techniques in positron emission tomography (PET) have led to the development of several ligands that bind to neurofibrillary tangles (NFT) and amyloïd-ß plaques. Shoghi-Jadid and colleagues were 
the first to publish on NFT and amyloïd- $B$ binding PET ligands (Shoghi-Jadid et al., 2002). The radioligand used in their study was 2-(1-\{6-[2-[18F]fluorethyl) (methyl)amino]-2-napthyl\}ethylidene)malononitrile ([18F] FDDNP), which binds to amyloïd- $B$ and NFTs in vivo. This study demonstrated a high accumulation of NFTs and amyloïd- $\beta$ in living $A D$ patients. Furthermore, the areas with a high [18F] FDDNP binding were the same areas that showed a low metabolism.

Another study, using Pittsburg Compound-B (PIB), an amyloïd- $\beta$ binding ligand, has also shown an inverse correlation between ligand binding and cerebral glucose metabolism (Klunk et al., 2004). Two other studies showed that PIB binding significantly discriminates between $A D, M C l$ and healthy controls (Jack et al., 2008; Kemppainen et al., 2007). The exact sensitivity and specificity of these PET ligand markers in discriminating between AD and other dementias is not clear yet. The development of ligands that can detect the neuropathological features of $A D$ provides a hopeful perspective on the diagnosis and early detection of AD. Furthermore, PET ligand imaging could become the ultimate biomarker with which to evaluate the effects of new amyloidal treatments.

\subsubsection{Structural neuroimaging: considerations}

The above-mentioned structural imaging studies show that atrophy of the medial temporal lobe structures, viz. hippocampus, parahippocampal cortex and entorhinal cortex, are the most consistent findings in AD. Atrophy of the structures in the medial temporal lobe has been shown to be sensitive diagnostic markers for Alzheimer's disease. VBM has been shown to be an accurate manner to assess atrophy in Alzheimer's disease and to evaluate structural changes over time, but it has its technical and methodological limitations. In therapeutic intervention studies it currently has no value since cholinesterase inhibitors do not change anatomy. In future drug studies VBM could be an important tool for the examination of anatomical changes. The recent developments of PET ligand imaging provide a new method that could become the ultimate diagnostic tool in the diagnosis and early detection of AD.

\subsubsection{Functional neuroimaging}

The previous section examined how brain structures change during the onset and progression of $A D$. In recent years several techniques have been developed that assess the functional activity of the brain. Two methods used to study cerebral activity in resting state are: single photon emission computational tomography (SPECT) and positron emission tomography (PET). Functional MRI ( $\mathrm{fMRI}$ ) is commonly measured during task performance. 


\subsubsection{SPECT}

In SPECT studies, Tc-99m-exametazime (Tc-99m-HMPAO) and I-123 IMP are the most commonly used radiopharmaceuticals to examine regional cerebral blood flow (perfusion). In AD hypo-perfusion is mainly shown in the parieto-temporal association areas. The frontal areas are mostly affected in the advanced stage of AD (Nestor et al., 2004). In their review, Nestor and colleagues show that abnormal cerebral blood flow is not a consistent finding in Alzheimer's disease. Nevertheless, a systematic review showed that decreased perfusion measured with SPECT has a good sensitivity (66-79\%) and specificity (70-91\%) in discriminating $A D$ patients from healthy controls, subjects with vascular dementia, and subjects with fronto-temporal dementia (Dougall et al., 2004). In predicting which $\mathrm{MCl}$ patients will progress to $A D$, several SPECT studies showed that hypo-perfusion has a high sensitivity (70-86\%) and specificity (70-80\%) (Cabranes et al., 2004; Encinas et al., 2003; Huang et al., 2002). Despite these high levels of sensitivity and specificity, the examination of the cerebral perfusion using SPECT is not of additional value to the differential diagnosis, certainly when it is performed in addition to structural imaging (Scheltens et al., 1997), because structural MRI images can provide more information about other possible causes of the dementia syndrome than SPECT.

\subsubsection{PET}

In PET the most commonly used method to assess regional cerebral metabolism is by using 2[18F]fluoro-2-deoxy-D-glucose (FDG). FDG binds in the cellular mitochondria and reflects glucose metabolism, which in turn reflects synaptic activity in nervous tissue. Results from PET studies are similar to those of SPECT studies and show reduced rCBF in the hippocampus, medial temporal lobes, parieto-temporal cortex and posterior cingulate cortex (Masdeu et al., 2005). Hypoperfusion in the posterior cingulate cortex, temporal cortex, and lateral association areas are consistent findings in SPECT and FDG-PET studies of early Alzheimer's disease and $\mathrm{MCl}$. Hypoperfusion in the temporal lobes and parieto-temporal lobes are consistent with neuropathological findings (Braak and Braak, 1991).

A recent study showed that FDG-PET has a high sensitivity and specificity in distinguishing AD from healthy elderly subjects (sensitivity $99 \%$, specificity $98 \%$ ), and in identifying Lewy body dementia (sensitivity $99 \%$, specificity $71 \%$ ) and fronto-temporal dementia (sensitivity $99 \%$, specificity $65 \%$ ) (Mosconi et al., 2008). Another study showed that PET is very sensitive (92\%) and specific (89\%) in predicting $A D$ in a $\mathrm{MCl}$ population (Drzezga et al., 2005). 
Although FDG-PET imaging provides valuable information, the additional value in a clinical environment is probably modest (Jagust et al., 2007).

\subsubsection{Functional MRI}

In addition to SPECT and PET, cerebral metabolism can also be measured by using functional magnetic resonance imaging ( $F M R I)$. In $F M R I$ the signal consists of the blood oxygen level dependent response (BOLD). The BOLD response is the hemodynamic response to changes in synaptic activity. FMRI is often used in psychology to examine the activity of brain structures during a neuropsychological test. In a clinical setting, there is no large role for fMRI at this moment.

In the case of Alzheimer's disease, fMRI has been used to examine the BOLD response during different memory tasks. The most consistent findings in memory tasks are a decreased BOLD response in the hippocampus, frontal cortex and temporal cortex se review by (Prvulovic et al., 2005). A difficult aspect of $\mathrm{fMRI}$ studies is the coupling between neuronal activity and the BOLD response. It has been demonstrated that small vascular lesions have an effect on the BOLD response (D'Esposito et al., 2003). Small vascular lesions are very common in normal aging, and can therefore induce a bias, certainly when groups of a different age are compared. In many fMRI studies where Alzheimer patients are compared to $\mathrm{MCl}$ and healthy elderly subjects, the average age of the AD patients is higher than in the other groups. Part of the difference in the BOLD response could be explained by the decline in coupling between synaptic activity and it's vascular response.

\subsubsection{Functional neuroimaging: considerations}

The results from SPECT and PET imaging show high levels of sensitivity and specificity in discriminating AD patients from healthy controls and other types of dementia. Nevertheless, these imaging methods do not provide significant additional information for the standard clinical evaluation, which includes structural imaging. In SPECT and FMRI a vascular signal is measured. This is based on the assumption of neurovascular coupling. In aging this neurovascular relation is disturbed, which makes it difficult to interpret these data.

\subsection{Electroencephalography}

Hans Berger was the first to apply the electroencephalogram (EEG) to the study of human subjects; EEG subsequently evolved into an excellent tool for the study of neurophysiology and pathologies of the central nervous system. EEG 
measures the electric potential difference that is generated by groups of firing neurons on the scalp. Other measures of functionality mostly examine synaptic function or glucose metabolism, both of which are indirect measures of synaptic activity. EEG is therefore the most direct measure of cortical activity.

Usually the EEG is measured in two domains i.e. the frequency and the time domain. In the frequency domain, changes in power of the frequency spectra are analysed. The EEG consists of four classic spectra, delta (1-4Hz), theta $(4-7 \mathrm{~Hz})$, alpha $(7-13)$, and beta $(14-30 \mathrm{~Hz})$. The gamma $(>30 \mathrm{~Hz})$ band was not studied extensively until the last decade. When electrodes on the scalp measure EEG, positive electric potential differences are counterbalanced by negative electric potentials. This results in an EEG signal on the scalp that is the sum of all the potential differences. When, for example, the state of arousal increases the synaptic activity in the brain also increases, resulting in dominance of the high frequencies in the EEG signal. The increment in synaptic activity also leads to increased noise in the EEG signal. The slow waves originate from deep brain areas. Thus, increased noise leads to a suppression of these signals on the scalp. This leads to a shift of power towards the higher frequencies. The analysis of frequency domains mainly measures changes in arousal. In neuropathologies the power spectra reflect the general condition of the brain. However, the source of the changed power spectrum cannot be located with satisfactory spatial resolution.

Measurements of the EEG in the time domain are generally performed by evoking potentials with a specific stimulus (i.e. the event-related potential (ERP)). The ERP provides information about the source of the EEG signal. In time domain studies, the stimuli are presented repeatedly so that the ERP can be separated from the noise of general brain activity. In the ERP complex, the amplitude and the latency are the measures that are examined most extensively. In this section, frequency analysis, event-related potential and coherence in Alzheimer's disease will be discussed.

\subsubsection{Frequency analysis}

One of the major hallmarks of EEG abnormalities in Alzheimer's disease is the general slowing down of the EEG signal, for review see (Jeong, 2004). The slowing down of the EEG signal is due to a decline in cortical brain activity, which mainly results in a decline of high-frequency signals. This decline of highfrequency activity leads to a relative increase of the slow-wave activity. This means that the spectral change is mostly expressed in terms of relative power changes in different frequency bands. The general slowing appears as a decrease in power in the faster alpha and beta band and as an increase in 
power in the delta and theta band. The decline in power in the alpha band is considered to be due to disconnection in the thalamic-cortical circuits. Several studies have demonstrated a correlation between changes in the power spectrum and the severity of disease (Jelic et al., 2000; Kowalski et al., 2001; Rodriguez et al., 1999).

The power shift to lower frequencies seems to be a consistent finding in Alzheimer's disease. However, several studies have also shown that changes in spectral power are not specific enough to discriminate $A D$ from all other causes of dementia. Especially the differentiation of $A D$ and vascular dementia (VaD) has proved to be difficult. Some studies have reported the differentiating potential of spectral power analysis, whilst others were unable to differentiate the two dementias (Jeong, 2004).

In discriminating $\mathrm{AD}$ from $\mathrm{MCl}$ subjects spectral changes have made differentiation between these groups possible with an accuracy of $78 \%$ (Huang et al., 2000). A longitudinal follow-up study of $\mathrm{MCl}$ shows changes in EEG spectral power in progressive $\mathrm{MCl}$ subjects, while there were no changes in stable $\mathrm{MCl}$ subjects after follow-up (Jelic et al., 2000). This study demonstrated that EEG power spectra have a predictive value in separating stable $\mathrm{MCl}$ subjects from progressive $\mathrm{MCl}$ subjects at baseline. The changes in the spectral profile together with parieto-temporal coherence showed a sensitivity of $77,8 \%$ and a specificity of $100 \%$.

Another longitudinal follow-up study has shown that the localisation and the amplitude of the alpha band provide a high discriminatory value between stable $\mathrm{MCl}$ and progressive $\mathrm{MCl}$ subjects at baseline (Huang et al., 2000). This evidence suggests that the changes in power spectra are sensitive to changes during the progression of AD pathology.

\subsubsection{Gamma band}

Gamma-band discharges have been reported in a variety of cognitive functions like attention, memory, olfaction, visual processes, object recognition and perception in animals and humans (Engel and Singer, 2001; Herrmann and Demiralp, 2005). Furthermore, other studies have reported that gamma-band synchronization correlates with cognitive functions, attention and short-term memory (Stopfer et al., 1997; Tallon-Baudry et al., 1998). High frequency discharges and synchronization seem to be responses to stimuli, and are thus involved in higher cognitive functions, as well as the binding of different features of a stimulus to a whole (Engel et al., 2001). 
Gamma-band oscillations occur spontaneously or can be evoked by different types of tasks. Steady-state gamma band oscillations can be obtained by the application of repeated clicks or tones with a frequency of $40 \mathrm{~Hz}$ (Galambos et al., 1981; Osipova et al., 2006; Ribary et al., 1991). Evoked gamma-band responses occur as temporary event-related oscillations. These oscillations occur simultaneously to ERPs. Several studies have reported decreased synchronisation of gamma-band oscillations in AD when compared to normal controls (Babiloni et al., 2004; Koenig et al., 2005). Changes in gamma band in AD have not been studied extensively, but changes in the gamma band have been demonstrated in normal aging. This supports the idea that analysis of the gamma band might be relevant for AD (Herrmann and Demiralp, 2005).

\subsubsection{Coherence analysis}

EEG coherence analysis is designed to examine whether brain waves from different brain areas are synchronized. Oscillatory synchronisation constitutes a fundamental mechanism for implementing coordinated communication between spatially distributed local networks in the brain (Schnitzler and Gross, 2005). This synchrony reflects the degree of functional connectivity between two or more cerebral areas. The synchrony between brain locations would tag the responses that code for one object and separate their responses from those neurons activated by other objects (Engel and Singer, 2001). Functional connectivity depends upon the integrity of anatomical connections in the neuronal network, but is also dependant on the neurotransmission in the neural network. A decrease in synchronisation may be indicative of a loss of functional integrity of the neural connections between different brain areas.

In $A D$, neuron loss leads to disconnection and loss of synchronisation between brain regions, for review see (Delbeuck et al., 2003). Decreased synchronisation in the alpha band is a well-described feature of $A D$ pathophysiology and is found mainly in the tempero-parietal and the fronto-parietal measures (Babiloni et al., 2004; Berendse et al., 2000; Jelic et al., 1997; Jelic et al., 1996; Locatelli et al., 1998). The relative loss in synchronisation is most evident in the long-distance fronto-parietal measures (Berendse et al., 2000; Locatelli et al., 1998).

Synchronization likelihood (SL) is a measure of the dynamic interdependencies between a time series (EEG channel) and one or more other time series. In contrast to coherence, SL measures linear and non-linear interdependencies, and it can do so as a function of time (Stam et al., 2002). Compared to normal aging subjects, $A D$ patients show a strong reduction of midline fronto-parietal SL in the alpha1 band and a reduction of the left fronto-parietal (F3-P3) SL in the delta 
band. In the right fronto-parietal (F4-P4) comparison a decline in SL has been shown in the theta band (Babiloni et al., 2004). In this study, VaD patients showed a global decline in SL over the whole scalp, which is most probably related to the vascular origin of this disorder.

The decrement in synchronization in $A D$ is not found in all frequencies. Locatelli et al. (1998) showed a decrease in alpha-band synchronisation and an increase in the delta-band synchronisation. In this study, the increased coherence in the delta band is explained as a lack of influence of subcortical cholinergic neurons on cortical activity. Stam et al. (2002) were the first to describe loss of coherence in the gamma band in AD. Synchronisation in the gamma band is considered to reflect processing of complex information and consciousness (Engel and Singer, 2001; Tallon-Baudry et al., 2004). It has been demonstrated that the level of desynchronization correlates to cognitive dysfunction in $A D$ (Stam et al., 2003).

It has been hypothesized that impaired synchronisation in AD is probably related to the loss of synaptic connectivity. However, the fact that impaired synchronization is not present in all frequency bands suggest that neurotransmitters are also involved (Stam et al., 2003). Despite these findings, the diagnostic value of synchronisation remains unstudied; no papers examining the sensitivity and specificity of synchronisation in AD were found.

\subsubsection{Neurotransmitters in frequency changes and coherence}

The general slowing of the EEG in AD has been attributed to cholinergic dysfunction. Several studies have demonstrated that ChEI leads to a significant reduction of slow-frequency power in AD patients (Adler et al., 2004; Rodriguez et al., 2002).

The correlation between the slowing of the EEG signal and the loss of coherence and loss of cholinergic neurons in the basal forebrain and the medial temporal lobe has been established (Holschneider et al., 1999). This study further demonstrated that cholinergic deafferentation results in loss of intra-hemispheric coherence in the $4-8 \mathrm{~Hz}$ band, which is restored after the administration of physostigmine, a cholinergic agonist.

Another animal study using cats, examined the cholinergic role in synchronisation by administration of the cholinergic antagonists scopolamine and carbachol, cholinergic agonist (Rodriguez et al., 2004). Scopolamine decreased EEG synchronisation while carbachol had an enhancing effect on synchronisation, which suggests that a major role is played by acetylcholine in the modulation of synchronous oscillations in the gamma band.

In humans scopolamine has a similar effect; it reduces resting-state 
synchronisation (Kikuchi et al., 2000). Other studies have demonstrated that cholinergic blockade by scopolamine leads to enhanced theta and delta power and attenuated alpha and beta power (Ebert and Kirch, 1998), as well as gamma power (Kikuchi et al., 2000; Rodriguez et al., 2004).

These findings suggest a major role for acetylcholine in synchronisation of brain activity. It is clear that synchronisation is affected by pharmacological manipulations of the cholinergic system in two directions. Drugs suppressing the cholinergic function reduce synchronisation while cholinergic agonists enhance synchronisation. Changes in the cholinergic nervous system cannot fully explain the changes in power spectra and coherence in AD. It is most likely that the changes in the power spectrum can be attributed to cholinergic dysfunction and loss of synaptic connections.

\subsubsection{Event-Related Potentials}

Event-related potentials (ERPs) are in the time domain of the EEG signal. ERPs can be divided into early potentials and late potentials. ERPs reflect cortical functioning, in terms of the efficiency of signal processing. The efficiency of signal processing depends mainly on the integrity of complex functional neuronal circuits. The early ERPs are considered a reflection of the neurological processing without cognitive processing of the stimulus. The late components are related to cognitive and perceptual processing.

ERPs are traditionally elicited by auditory or visual oddball paradigms, but can also be measured during a variety of cognitive tasks.

\subsubsection{Early ERPs}

P50

The P50 is mostly studied in sensory gating paradigms in which two identical stimuli are presented 500 ms after each other.

Several studies showed that in a sensory gating paradigm the second peak is higher in AD than in controls (Cancelli et al., 2006; Jessen et al., 2001). A study with healthy first-degree family members of $A D$ patients showed similar results (Boutros et al., 1995). This increased P50 amplitude after the second stimulus has been related to impaired cortical inhibition in AD.

In oddball paradigms the P50 has also been shown to be enhanced in $\mathrm{AD}$ and $\mathrm{MCl}$ (Golob et al., 2002; Golob and Starr, 2000). Another study that also used the oddball paradigm, showed that when stable $\mathrm{MCl}$ patients are compared to converted $\mathrm{MCl}$ patients, the P50 amplitude of the $\mathrm{MCl}$ converters is increased (Golob et al., 2007). Despite these findings, the diagnostic sensitivity and specificity of the P50 remain unstudied. 
N100

The N100 is a large negative component with a mean latency of $100 \mathrm{~ms}$. This component can be evoked by different paradigms. The latency and amplitude are affected by attention. The N100 amplitude is thought to reflect perceptual capacity and an orientation response. The N100 complex can be observed after the presentation of unexpected stimuli. Several studies have demonstrated a smaller N100 amplitude in AD patients compared to controls (Boutros et al., 1995; Polich et al., 1990; Szelies et al., 1995). Other studies demonstrated that there was no difference in N100 amplitude between healthy controls and AD patients (Golob and Starr, 2000; Ito et al., 1990). The study by Szlelies (1995), demonstrated increased latency of the N100 component, while the other studies did not demonstrate this difference. The diagnostic sensitivity and specificity of the N100 component remain unstudied.

\section{MISMATCH-NEGATIVITY}

Mismatch-Negativity (MMN) is a specific auditory ERP that originates in the auditory cortex and represents auditory sensory memory. The MMN is evoked in response to unattended, infrequent deviant tones and indexes pre-attentive stimulus in comparison to an existing memory trace (Naatanen et al., 1987). It has been shown that MMN is intact in $A D$, but that the amplitude of MMN is decreased in AD when compared to healthy controls (Gaeta et al., 1999; Kazmerski et al., 1997). Similar results are found when a cholinergic antagonist is administered in young healthy individuals, suggesting a cholinergic role in the regulation of auditory sensory memory (Pekkonen et al., 2001). No studies exist that examined the potential of MMN to differentiate between different causes of dementia.

\subsubsection{Late ERPs}

N200

The N200 is evoked by changes in the physical properties of a stimulus, which are task relevant when a subject focuses attention. The N200 amplitude depends on task-related factors like categorization and attention.

Several studies have demonstrated that the N200 latency is significantly increased in AD when compared with healthy controls (Patterson et al., 1988; Szelies et al., 1995; Verleger et al., 1992). Other studies did not demonstrate a difference in N200 latency or amplitude between AD and healthy controls (Golob et al., 2001; Polich et al., 1990). Longer N200 latencies were also demonstrated in vascular cognitive impairment (van Harten et al., 2006). This suggests that a longer N200 latency is not a specific marker for AD. Its diag- 
nostic accuracy in discriminating between AD and other causes of dementia has not been studied.

P300

The P300 is the ERP with the highest amplitude and is the most extensively studied ERP. It is elicited by simple auditory or visual discrimination tasks. It is widely accepted that P300 potentials result from intra-cortical currents induced by postsynaptic potentials. The P300 amplitude is considered as the manifestation of brain activity that reflects attention to incoming stimulus information when representations are updated. The P300 latency is considered as an index for stimulus classification speed.

Several groups have studied P300 changes in Alzheimer's disease with consistent results; the P300 shows a longer latency and lower amplitude in AD (Golob et al., 2001; Golob and Starr, 2000; Patterson et al., 1988; PokryszkoDragan et al., 2003; Polich and Corey-Bloom, 2005; Polich and Herbst, 2000; Polich et al., 1990; Szelies et al., 1995). A study by Frodl et al. (2002) showed that the P300 latency and amplitude together have a high level of sensitivity (90\%) and specificity $(76.9 \%)$ in discriminating $A D$ patients from healthy controls. Furthermore, this study addressed the fact that when considered separately P300 amplitude and latency do not reach this level of sensitivity (Frodl et al., 2002). The discrimination between $A D$ and vascular dementia is difficult to make solely on the basis of P300 amplitude or latency (Neshige et al., 1988).

One longitudinal follow-up study reported a significant difference in P300 latency between AD subjects and normal controls and between $\mathrm{MCl}$ patients and patients with dementia of another type, in a $\mathrm{MCl}$ cohort at baseline. The group of progressive $\mathrm{MCl}$ subjects demonstrated significantly longer P300 latencies, while no differences in P300 amplitude were reported. The sensitivity $(52,9 \%)$ and specificity $(76,9 \%)$ in discriminating stable from progressive $\mathrm{MCl}$ subjects did not reach a satisfying level (Gironell et al., 2005).

\subsubsection{Neurotransmitters in ERPs}

The information about the cholinergic role in ERP components is important if ERPs are to be used in clinical trials of cholinergic intervention.

The use of ChEl in AD patients leads to a decrease in P300 latency (Katada et al., 2003; Werber et al., 2001). Two other studies demonstrated similar results and furthermore showed a strong correlation between the decreased P300 latency and cognitive performance as measured by ADAS-cog (Onofrj et al., 2002; Thomas et al., 2001). None of these studies reported an effect of ChEl's on P300 amplitude, which suggests that P300 latency is more sensitive to therapeutic change. 
Other evidence for the cholinergic involvement in ERPs comes from studies using scopolamine as a model for cholinergic dysfunction. Scopolamine induces increased latency of the pre-attentional P50 and N100 (Pekkonen et al., 2005) as well as of the P300 (Kikuchi et al., 2000). These data suggest that the ERP latency is sensitive to changes in cholinergic neurotransmission.

In sum, P300 latency provides a sensitive and objective marker of cognitive function that is sensitive to changes in cholinergic neurotransmission.

\subsubsection{EEG: considerations}

Different EEG paradigms showed the ability to discriminate AD patients from normal elderly subjects. Many studies report high levels of sensitivity and specificity in discriminating $A D$ subjects from healthy controls, but do not report on the discrimination between different causes of dementia. Especially the discrimination between $A D$ and vascular dementia seems difficult to make on the basis of EEG changes.

A major problem with EEG studies is that there are no standards concerning the techniques and paradigms used. There is a large variance in the techniques used for every paradigm. The auditory oddball paradigm is the only paradigm discussed that is performed with commensurability between the discussed studies. The large difference between the paradigms used makes it difficult to evaluate which paradigm is the most sensitive and specific for AD. Another difficulty is the large variability of the patients and control groups used in the EEG studies. To clarify which paradigm performs optimally in discriminating between patient groups, head-to-head comparison studies of different EEG paradigms should be conducted.

\subsection{Discussion}

The main purpose of this review was to discuss the value of EEG as a marker for $A D$ in comparison to other markers. An overview of the critical aspects of these markers is presented in table 1. Three aspects of the role of EEG as a surrogate marker in Alzheimer's disease are discussed in this section. First of all, its diagnostic accuracy and contribution to currently used markers is examined. Secondly, its sensitivity in discriminating progressive $\mathrm{MCl}$ patients from stable $\mathrm{MCl}$ patients is determined, as this could be important for the early treatment of AD. Thirdly, its value as an endpoint marker for therapeutic changes will be evaluated. 


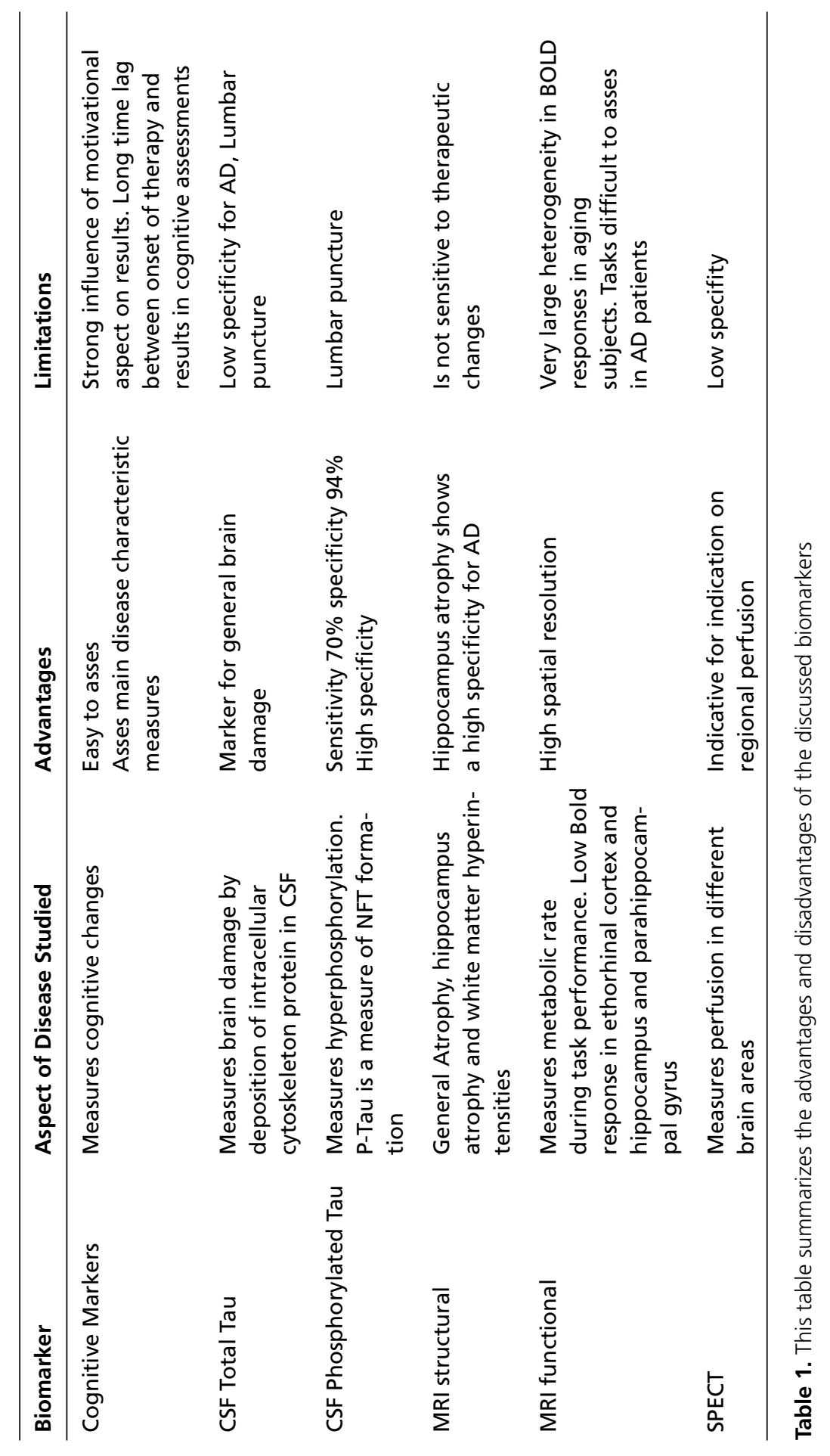


Functional and structural brain markers of Alzheimer's disease | Chapter 2

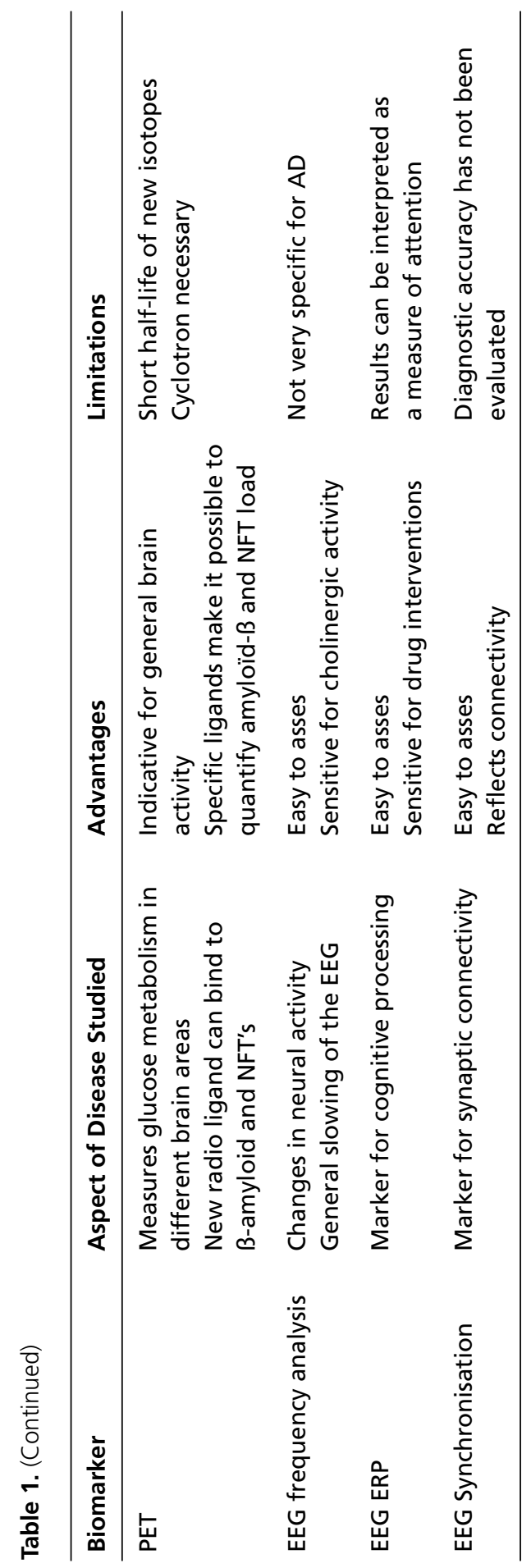




\subsubsection{Diagnostic accuracy}

When evaluating EEG as a diagnostic marker it is important to consider whether EEG is more sensitive or specific than commonly used diagnostic measures.

The diagnostic specificity of EEG is not sufficient to replace commonly used markers such as cognitive tests and structural neuroimaging, since these provide more information about the cause of dementia. Therefore, the conclusion is that EEG does not significantly contribute to diagnostic accuracy in the detection of $A D$.

\subsubsection{Early detection}

The second aspect under consideration regards the sensitivity of EEG to detect early changes in the progression of $\mathrm{MCl}$ to $\mathrm{AD}$. As was pointed out above, it should be noted that $\mathrm{MCl}$ is a concept that includes a heterogeneous population. A large part of the subjects with $\mathrm{MCl}$ will not develop AD (Larrieu et al., 2002).

Analysis of the spectral profile has been shown to be effective in discriminating stable $\mathrm{MCl}$ subjects from progressive $\mathrm{MCl}$ subjects (Huang et al., 2000; Jelic et al., 2000). The value of ERP studies in discriminating stable $\mathrm{MCl}$ subjects from progressive $\mathrm{MCl}$ subjects has proved to be poor. The predictive accuracy of biochemical markers in CSF proved to be higher than that of EEG. In these studies the predictive sensitivity of decreased levels of amyloïd- $\beta$ in the CSF was $59 \%$ and the specificity was $100 \%$. Analysis of t-tau showed even higher levels of sensitivity and specificity, viz. $83 \%$ and $100 \%$ respectively.

Functional and structural MRI did not exceed these levels of sensitivity and specificity. However, this does not imply that there is no role for EEG as a predictive marker in $\mathrm{MCl}$. Future studies should be carried out to examine the role of EEG coherence as a marker of progression. Furthermore, it has been shown that changes in the spectral profile show satisfactory sensitivities in the early detection of AD. Another advantage of EEG in comparison to biochemical measures is the fact that EEG is less invasive than a lumbar puncture.

\subsubsection{Therapy evaluation}

To evaluate whether EEG is a valuable endpoint marker of therapeutic changes depends on the target of the drug. At present, cognitive and behavioural markers are most frequently used as endpoints in clinical trials. Cognition is mostly evaluated by using the ADAS-Cog (Rosen et al., 1984), which is a clinically meaningful outcome measure. Disadvantages of ADAS-cog as an endpoint marker relate to the subjective nature of several of its items and its insensitivity for cognitive decline in placebo groups (Wesnes, 2008). This implies that using 
ADAS-cog as an endpoint marker requires large study samples and a very long follow-up period. Furthermore, cognitive markers are very sensitive for confounding factors such as mood and motivational aspects.

At present there are no disease-modifying drugs available that suppress the neurodegenerative process in AD. Especially structural imaging markers would be of interest to monitor the effect of these types of drugs. Currently, most AD patients are treated with symptom suppressing cholinesterase inhibitors. EEG has proved to be a very sensitive marker for changes in cholinergic activity. In the case of $A D$ a major part of the general slowing down of the EEG can be attributed to cholinergic dysfunction. Healthy subjects also show a general slowing of the EEG signal after the administration of scopolamine. Scopolamine is a muscarine receptor antagonist that is often used as a pharmacological model for $A D$; it shows significant changes in the EEG that are quite similar to the changes in AD (Ebert and Kirch, 1998; Kikuchi et al., 1999). Cholinesterase inhibitor treatment in $A D$ has reduced the general slowing of the EEG spectra (Adler et al., 2004; Rodriguez et al., 2004). These findings indicate that analysis of the frequency spectrum is a sensitive marker of changes in cholinergic activity.

It has been demonstrated that ERPs, and especially the P300, are sensitive to changes in cholinergic neurotransmission. Scopolamine-induced cholinergic blockade leads to increased latency, while P300 latency has shown to decrease after ChEl treatment (Katada et al., 2003; Onofrj et al., 2002; Thomas et al., 2001; Werber et al., 2003). The P300 latency and amplitude are vulnerable for level of attention. Cholinergic stimulation is known to increase the level of attention, which could partly explain the decreased latency after cholinergic stimulation (Polich and Herbst, 2000).

Viewed together these results suggest that P300 is a sensitive marker of changes due to therapeutic intervention. Thus, when psychopharmacological interventions have to be evaluated, EEG is probably an interesting candidate biomarker.

\subsection{Conclusions}

This review demonstrates that EEG is not a valuable diagnostic marker for $A D$, since it lacks diagnostic specificity. With regard to the early detection of $A D$, frequency analysis has proved to be sensitive and specific for $A D$, but biochemical markers are more sensitive than EEG. Furthermore, it has been demonstrated that EEG is a good candidate marker for the evaluation of 
therapeutic intervention. The main advantage of EEG is the direct way by which neurophysiology is measured. Moreover, EEG is a cost-effective, easy-to-assess and non-invasive method in comparison to other markers such as SPECT and MRI markers.

In spite of these advantages some problems remain, as there is a large technical discrepancy between EEG studies. Hence, consensus is lacking about how paradigms should be performed technically and how they should be analysed. Furthermore, a head-to-head comparison between EEG paradigms in the same population in order to evaluate which paradigms are most sensitive and specific for $A D$ and for changes after therapeutic interventions has, as yet, never been carried out. Future research should focus on a head-to-head comparison of different biomarkers in order to evaluate their sensitivity and specificity. 



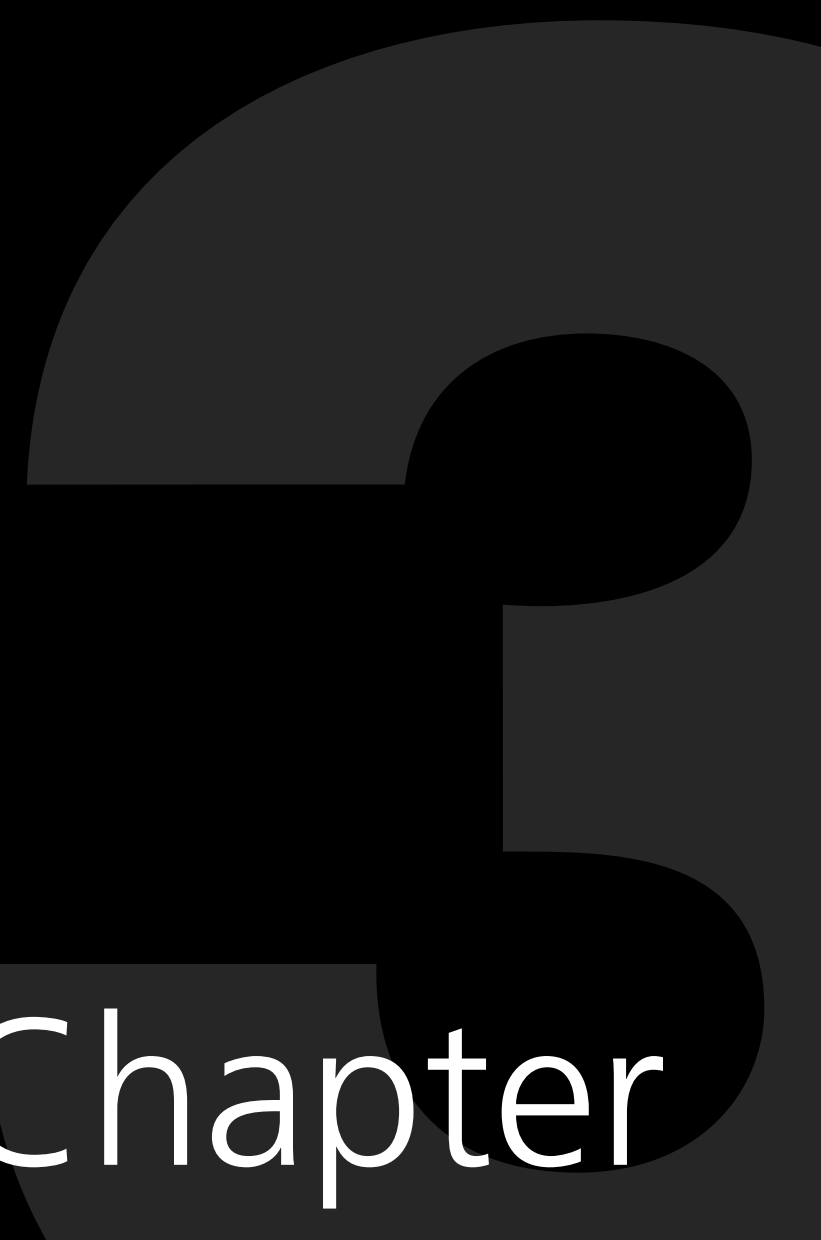





\title{
Increased EEG gamma band activity in Alzheimer's disease and mild cognitive impairment
}

This chapter was published as:

J.A. van Deursen, E.F.P.M Vuurman, F.R.J. Verhey, V.H.J.M. van Kranen-Mastenbroek, W.J. Riedel (2008). Journal of Neural Transmission 115 (9): 1301-11

\begin{abstract}
Background: High frequency $(30-70 \mathrm{~Hz})$ gamma band oscillations in the human electro-encephalogram (EEG) are thought to reflect perceptual and cognitive processes. It is therefore interesting to study these measures in cognitive impairment and dementia.

Aims: To evaluate gamma band oscillations as a diagnostic biomarker in Alzheimer's disease (AD) and mild cognitive impairment (MCI).

Methods: Fifteen psychoactive drug naïve AD patients, twenty $\mathrm{MCl}$ patients and twenty healthy controls participated in this study. Gamma band power (GBP) was measured in four conditions; resting state, music listening, story listening and visual stimulation. To evaluate test-retest reliability (TRR), subjects underwent a similar assessment one week after the first.

Results: The overall TRR was high. Elevated GBP was observed in AD when compared to $\mathrm{MCl}$ and control subjects in all conditions.

Conclusion: The results suggest that elevated GBP is a reproducible and sensitive measure for cognitive dysfunction in $A D$ in comparison with $\mathrm{MCl}$ and controls.
\end{abstract}




\section{Introduction}

Alzheimer's disease (AD) is a progressive neurodegenerative disorder with clinical manifestations of loss of cognitive functions and impaired daily life activities.

The neurophysiological basis for the cognitive and behavioural dysfunction in $A D$ is not completely understood but involves changes in a variety of neural substrates throughout the brain. Frequency analysis of ongoing (background) electroencephalography (EEG) provides a good indication about the neurological integrity of the central nervous system (CNS) and has as such often been used in $A D$ studies. In clinical studies the frequency spectrum of EEG is divided in four frequency bands; delta $(<4 \mathrm{~Hz})$, theta $(4-8 \mathrm{~Hz})$, alpha $(8-13 \mathrm{~Hz})$ and beta (13-30 Hz). The EEG in AD is characterised by an increase of power in the lower frequencies and a decrease in the power of the higher frequencies (Huang et al., 2000; Jelic et al., 1996; Jeong, 2004). The decline in power of the higher frequencies is a consistent finding in $A D$ that correlates with cognitive performance, but nevertheless has no etiological specificity for $A D$. It is especially difficult to discriminate $A D$ from vascular dementia (VaD), based on clinical EEG (Jeong, 2004).

A basic question in functional brain research is how the manifold of serial and parallel neuronal activations needed to process basic stimuli are integrated and bound together. The precise timing and integration of neural activation is crucial for successful information processing in the brain. Several studies have suggested an important role for very high frequency or gamma band activity (30-100 Hz) in the timing and integration process of neuronal networks in the brain (Basar et al., 2001; Kaiser and Lutzenberger, 2003; Singer and Gray, 1995). Gamma band oscillations can thus be considered as neural activity that is generated to synchronize sub-processes in neural networks that are involved in specific information processing (Kaiser and Lutzenberger, 2003; Kaiser and Lutzenberger, 2005). High frequency discharges and synchronization seem to be responses to stimuli, as a mechanism for the integration of different features of a stimulus to a whole (Tallon-Baudry and Bertrand, 1999). As reviewed by Tallon-Baudry \& Bertrand (1999) there are three ways to describe gamma band activity; 1) The gamma band evoked response which is characterized by EEG activity that is phase-locked to the onset of a certain stimulus and is therefore directly related to it. 2) The $40 \mathrm{~Hz}$ steady-state response (SSR) which is a sinusoidal response at the driving stimulus frequency and is elicited by simple visual, auditory or somatosensory stimuli. This SSR can be interpreted as a natural resonance frequency of the brain and is related to primary sensory processing (Galambos et al., 1981). 3) Induced gamma band oscillations which 
result from continuous sensory stimulation and are not time or phase locked to a specific stimulus but elevated during meaningful information processing. Taken together, gamma band activity and synchronicity play an important role in both primary stimulus processing as well as higher information processing.

In Alzheimer's disease several studies have reported decreased synchronization of the gamma band (Koenig et al., 2005; Stam et al., 2002; Stam et al., 2003) but none of these human studies have focused on induced gamma band power (GBP). EEG power expresses the magnitude and intensity of the local EEG signal and is calculated by the squared amplitude of the oscillations. Synchronization is a numerical property of the association between two or more different EEG electrodes requiring groups of neurons distant from each other firing in the same phase and/or frequency. In comparison to synchronization, EEG power could be a simpler, faster and more straightforward measure to examine changes in gamma band oscillations in patient groups. Furthermore, most studies on gamma band activity in $A D$ have been assessed in patients using psychoactive medication. To rule out these potential medication effects as confounding with diagnosis, only patients naïve for psychoactive drugs should be included in studies comparing gamma band activity between patients and controls.

The current study focuses on induced gamma band power and has two major objectives. The primary objective is to evaluate the diagnostic sensitivity of induced GBP in discriminating psychoactive drug naïve $A D$ patients from $\mathrm{MCl}$ patients and healthy controls. The secondary objective is to evaluate the reliability and stability of induced GBP in cognitively impaired patients. The design for the first objective is based on a study by Fitzgibbon et al. (2004), which showed gamma band augmentation in a variety of relatively simple cognitive tasks in healthy volunteers (Fitzgibbon et al., 2004). Music listening, story listening and movie watching are highly over-learned tasks which makes them particularly suitable to be applied in cognitively impaired patients.

In the current study we determined whether differences in GBP, induced by the cognitive task load of these paradigms, could discriminate AD patients, $\mathrm{MCl}$ patients and healthy controls. Since information processing is disturbed in $A D$ and $\mathrm{MCl}$ we hypothesised that induced GBP would be lower in AD subjects than in $\mathrm{MCl}$ and controls subjects in all tasks conditions. Furthermore it was to be expected that GBP in $\mathrm{MCl}$ was lower than in the control group and higher than in the AD group. 


\section{Materials and Methods}

\section{Subjects}

The study involved three different groups of subjects:

1. Fifteen psychoactive drug naïve patients with a diagnosis of probable $A D$ according to the NINCDS-ADRDA criteria (McKhann et al., 1984) were included. Standard blood workup and neuroimaging (CT or MRI) were carried out, and the diagnosis was supported by abnormal performance on neuropsychological testing.

2. Twenty psychoactive drug naïve patients with a diagnosis of $\mathrm{MCl}$ according to the Petersen criteria were included (Petersen et al., 2001). $\mathrm{MCl}$ subjects also received standard blood workup, neuro-imaging and neuropsychological testing. The diagnosis $\mathrm{AD}$ or $\mathrm{MCl}$ was made in a weekly consensus meeting of different specialists.

3. Twenty healthy control subjects were recruited from the Maastricht Aging Study (MAAS), a longitudinal study of the determinants of healthy cognitive aging (van Boxtel et al., 1998). The control subjects were not using any psychoactive medications. The medical history was screened by a medical health questionnaire. Main exclusion criteria were a history of stroke, head trauma, or any other neurological or psychiatric disorders. Additional exclusion criteria were severe cardiovascular disease, a Hachinski Ischemic Scale (HIS) (Hachinski et al., 1974; Rosen et al., 1980) higher than three or a history of substance abuse and/or other serious system diseases (e.g. malignancy, uncontrolled hypertension, neuropathy or seizure disorders). All $A D$ and $\mathrm{MCl}$ patients were recruited at the Memory Clinic of University Hospital Maastricht and were judged competent to give consent by their treating physicians. This study was carried out in accordance with the declaration of Helsinki. All participants gave written informed consent prior to the study, for AD patients a family member also signed the consent form. The local Medical Ethics Committee of University Hospital Maastricht approved the study.

\section{Experimental procedure}

All subjects took part in two identical recording sessions, spaced apart one week. Prior to EEG acquisition, the cognitive subscale of the Alzheimer's Disease Assessment Scale (ADAS-cog) (Rosen et al., 1984; Verhey et al., 2004) was administered, on both occasions. The Dutch version of the National Adult Reading Test (NART) (Schmand et al., 1991) was administered to estimate pre-morbid intelligence. The three outcome measures; music listen- 
ing, story listening and movie watching were part of a larger study, which further included: auditory $40-\mathrm{Hz}$ steady state stimulation, contingent negative variation (CNV), a checkerboard reversal task and an event related potentials (ERP) paradigm. Continuous EEG was recorded while subjects performed the music listening task, story listening task and movie watching task. Headphones were used to present the auditory stimuli. To evaluate test-retest reliability (TRR) of the acquired data, all subjects were tested twice with an interval of at least one week.

\section{EEG acquisition}

A commercially available Nuamps ${ }^{\circledR}$ EEG acquisition system was used to record the EEG's. Electrodes $(\mathrm{Ag} / \mathrm{AgCl})$ were positioned following the 10-20 system on a 32-channel electrode cap, using 19 Medcat $^{\circledR}$ electrodes. A low pass filter of $100 \mathrm{~Hz}$ and a high pass filter of $0,1 \mathrm{~Hz}$ were applied. The sample frequency was $512 \mathrm{~Hz}$ and analogue-digital conversion was $20 \mathrm{bit}$. Electrode impedance was kept below $5 \mathrm{k} \Omega$. A reference electrode was placed on the right ear lobe. To control for possible vertical eye movements, an electro-oculogram (EOG) electrode was placed one centimetre under the midline of the right eye. A ground electrode was placed on the forehead, at Fpz. EEG's were recorded in a magnetically shielded and sound attenuated room. The visual stimuli were presented on a flat, LCD screen located outside the shielded room at eye height. Subjects were able to see the screen through magnetically shielded glass. Neuroscan ${ }^{\circledR} 4.3$ software was used for EEG recording and analyses.

EEG Gamma band paradigms

During the resting state measurement, subjects were instructed to keep their eyes open and fixate at a white crosshair located on a black screen for 90 seconds.

During the music listening task, subjects were instructed to listen for 90 seconds to broad-spectrum acoustical music. While listening to the stimuli subjects fixated on a white crosshair on a black screen and kept their eyes open. The music was a segment from Enya's "From where I am".

During the story listening task subjects listened for tree minutes to a fragment of a Dutch spoken documentary (Fly Away 2, fragment Australia). While listening to the stimuli subjects had to fixate on a white crosshair on a black screen with their eyes open. The fragment was spoken monotonously, and pauses were not longer than five seconds. Subjects were instructed to listen carefully to the spoken text. 
In the visual task a series of moving abstract objects were presented for 180 seconds. The objects were coloured and moved at variable speed. This was a fragment of recorded Windows ${ }^{\circledR}$ screensaver. Subjects were instructed to monitor the movements carefully.

\section{EEG analysis}

EEG analysis was performed off-line. The continuous EEG files were cut in epochs of one second. Epochs containing eye-movement, electromyographic activity and head motion artefacts were excluded from analysis. The average number of uncontaminated epochs in resting state condition was 62 (AD: $55, \mathrm{MCl}: 63$, Control: 67). In the music listening task the average number of uncontaminated epochs was 67 (AD: 60, MCl: 67, Control: 74). In the story listening task the average number of uncontaminated epochs was 153 (AD: 145, MCl: 161, Control: 153). In the visual task the average number of uncontaminated epochs was 85 (AD: $83, \mathrm{MCl}: 89$, Control: 84). The uncontaminated epochs were transformed from the temporal domain to the frequency domain using fast-Fourier transformation $(1 \mathrm{~Hz}$ resolution, 512 point block-size, Hanning window 1-100 Hz). The gamma band was defined as the frequency between $30-100 \mathrm{~Hz}$. A notch filter of $50 \mathrm{~Hz}$ was applied to omit contamination of the electrical light network in the room. The frequency epochs were averaged and absolute GBP was calculated for every task, subject and electrode.

\section{EMG analysis}

In order to ensure that the results are related to EEG differences and not to electromyographic (EMG) contamination (Whitham et al., 2007), an extra analysis was performed on those electrodes that previously showed GBP differences between the groups. The procedure involved low-pass amplitude filtering and visual inspection of the EEG epochs under guidance of a clinical EEG expert. Obvious and suspect epochs for EMG contamination were omitted from further analysis. The number of 'clean' epochs was in resting state 53 (AD: 43, MCl: 57, Control: 61 ), in music listening 56 (AD: 52, MCl: 64, Control: 52), in story listening 131 (AD: 128, MCl: 135) and in the visual task 68 (AD: 71, MCl: 68, Control: 64). These 'corrected' EEG epochs were further processed and analysed in the same manner as the original data. A paired samples t-test was used to examine differences between the EMG corrected an uncorrected data.

\section{Statistics}

Statistical analysis was done with SPPS for Windows (version 11.5). Significance levels associated with the differences in GBP between the $A D, \mathrm{MCl}$ and control groups were calculated for each electrode using ANOVA for repeated measures. Electrode 
positions were used as a within subject variable. Since GBP was calculated in two identical sessions, the mean of these two sessions was used to calculate between group differences. Post-Hoc Bonferonni correction for multiple comparisons was used. For the ANOVA for repeated measures analysis, the mean EEG power values of the two sessions were calculated for each subject and each electrode.

To examine test-retest reliability of GBP within the different paradigms, a paired T-test was used to evaluate potential differences between the two sessions for the $\mathrm{Fz}, \mathrm{Cz}$ and $\mathrm{Pz}$ electrodes. Furthermore Pearson's correlation coefficient was calculated for each task at Fz, Cz and Pz. These analyses were done for each patient group separately.

To examine the relation between GBP and cognitive performance measured by ADAS-cog, Pearson's correlation coefficient was calculated between GBP and ADAS-cog.

To test whether the stimuli induced increased GBP, these conditions were compared to resting state using ANOVA. The differences between the groups in changes in GBP in task condition compared to resting state were examined by calculating the ratio between task and resting state. Differences in this ratio between groups were analysed using ANOVA for repeated measures.

\section{Results}

\section{Subject's demographics}

Fifteen AD patients (11 male, 4 female) with a mean age (SD) of $75.2 \mathrm{yrs}(6.9)$ and a mean (SD) MMSE score of 20.8 (2.7, range 17-26), twenty (12 male, 8 female) MCI patients with a mean (SD) age of 70.6 (7.2) and mean (SD) MMSE of 26.3 (1.6, range 23-29) and, twenty (12 male, 8 female) healthy control subjects with a mean (SD) age of 69.5 (6.1) and mean (SD) MMSE of 29.3 (0.8, range 28-30) completed the study. There was no statistical difference in age between the groups $\left(F_{2,52}=2.2, p=.14\right)$.

\section{Neuropsychological tests}

Average ADAS-Cog scores were 19,6 (SD: 5.9; $n$ 15) in the AD group, 10.8 (SD: 4.5; $\mathrm{n} \mathrm{20)}$ in the $\mathrm{MCl}$ group and 5.8 (SD: 2.6; $\mathrm{n} 20$ ) in the control group. These scores showed a significant difference between the groups in the expected direction $\left(F_{2,52}=84.8, p<.001\right)$. Results from the NART showed that the mean estimated pre-morbid intelligence was $96,9\left(F_{2,52}=2.3, p=1.03\right)$ and did not differ between the groups. 


\section{Test retest reliability (TRR)}

The paired samples T-test revealed a significant difference between the sessions in the resting state condition in the $A D$ group at electrode $C z$ and in the control group at $\mathrm{Cz}$ and $\mathrm{Pz}$ electrode (see table 1). In the other task conditions there were no significant differences between the two sessions.

Pearson's correlation coefficient between the two sessions was very high in resting state, music listening, and story listening for all the groups (see table 1). The visual task showed a lower correlation between the sessions for all the groups.

\section{Induced gamma band paradigms}

Figure 1 summarizes the results of GBP in the four conditions.

In resting state, GLM showed differences between the group for $\mathrm{C3}\left(\mathrm{F}_{2,52}=\right.$ 3.83, $\mathrm{p}=.037), \mathrm{P} 3\left(\mathrm{~F}_{2,52}=4.29, \mathrm{p}=.032\right), \mathrm{Pz}\left(\mathrm{F}_{2,52}=5.26, \mathrm{p}=.009\right), \mathrm{P} 4\left(\mathrm{~F}_{2,52}=4.51\right.$, $\mathrm{p}=.039), \mathrm{T} 6\left(\mathrm{~F}_{2,52}=4.10, \mathrm{p}=.020\right), \mathrm{O} 1\left(\mathrm{~F}_{2,52}=3.42, \mathrm{p}=.044\right)$ and $\mathrm{O} 2\left(\mathrm{~F}_{2,52}=6.33\right.$, $\mathrm{p}=.006$ ). Bonferonni correction for multiple comparisons showed that GBP was higher in the $A D$ group compared to the $\mathrm{MCl}$ at $\mathrm{C} 3$ and $\mathrm{O} 2$ and control group at $\mathrm{C} 3, \mathrm{P3}, \mathrm{Pz}, \mathrm{P} 4, \mathrm{~T} 6, \mathrm{O} 1$ and $\mathrm{O} 2(\mathrm{p}<.05)$. There was no difference between the $\mathrm{MCl}$ and control group.

In the music listening task, GLM showed differences between the groups for T5 $\left(F_{2,52}=5.3, p=.006\right), T 6\left(F_{2,52}=5.2, p=.012\right), P 3\left(F_{2,52}=7.13, p=.021\right), P z$ $\left(F_{2,52}=7.02, p=.001\right), O 1\left(F_{2,52}=9.18, p<.004\right)$ and $O 2\left(F_{2,52}=7.38, p<.002\right)$. Bonferonni correction for multiple comparisons showed higher GBP in the AD group when compared to the $\mathrm{MCl}$ group at $\mathrm{P} 3, \mathrm{O} 1, \mathrm{O} 2$ and control group at $\mathrm{T5}, \mathrm{T6}, \mathrm{P3}, \mathrm{Pz}, \mathrm{O} 1$ and $\mathrm{O} 2(\mathrm{p}<.05)$. There was no difference between $\mathrm{MCl}$ and control subjects.

In the story listening task, GLM showed a difference between the groups for $\mathrm{O} 2\left(\mathrm{~F}_{2,52}=7.23, \mathrm{p}=.003\right)$. Bonferonni correction for multiple comparisons showed significantly higher GBP in the AD group when compared to the control group. There was no difference between the $\mathrm{MCl}$ group and the control group. There was no significant difference between the groups at other electrodes.

In the visual task, ANOVA showed a difference between the groups for T5 $\left(F_{2,52}=4.13, p=.029\right), O 1 \quad\left(F_{2,52}=5.16, p=.022\right)$ and $O 2\left(F_{2,52}=6.82, p=.001\right)$. Bonferonni correction for multiple comparisons showed significantly higher $\mathrm{GBP}$ in $\mathrm{AD}$ compared to $\mathrm{MCl}$ at $\mathrm{O} 2$ electrode and control group at $\mathrm{T5}, \mathrm{O} 1$ and $\mathrm{O} 2$ $(p<.05)$. There was no significant difference between $\mathrm{MCl}$ and control group. 


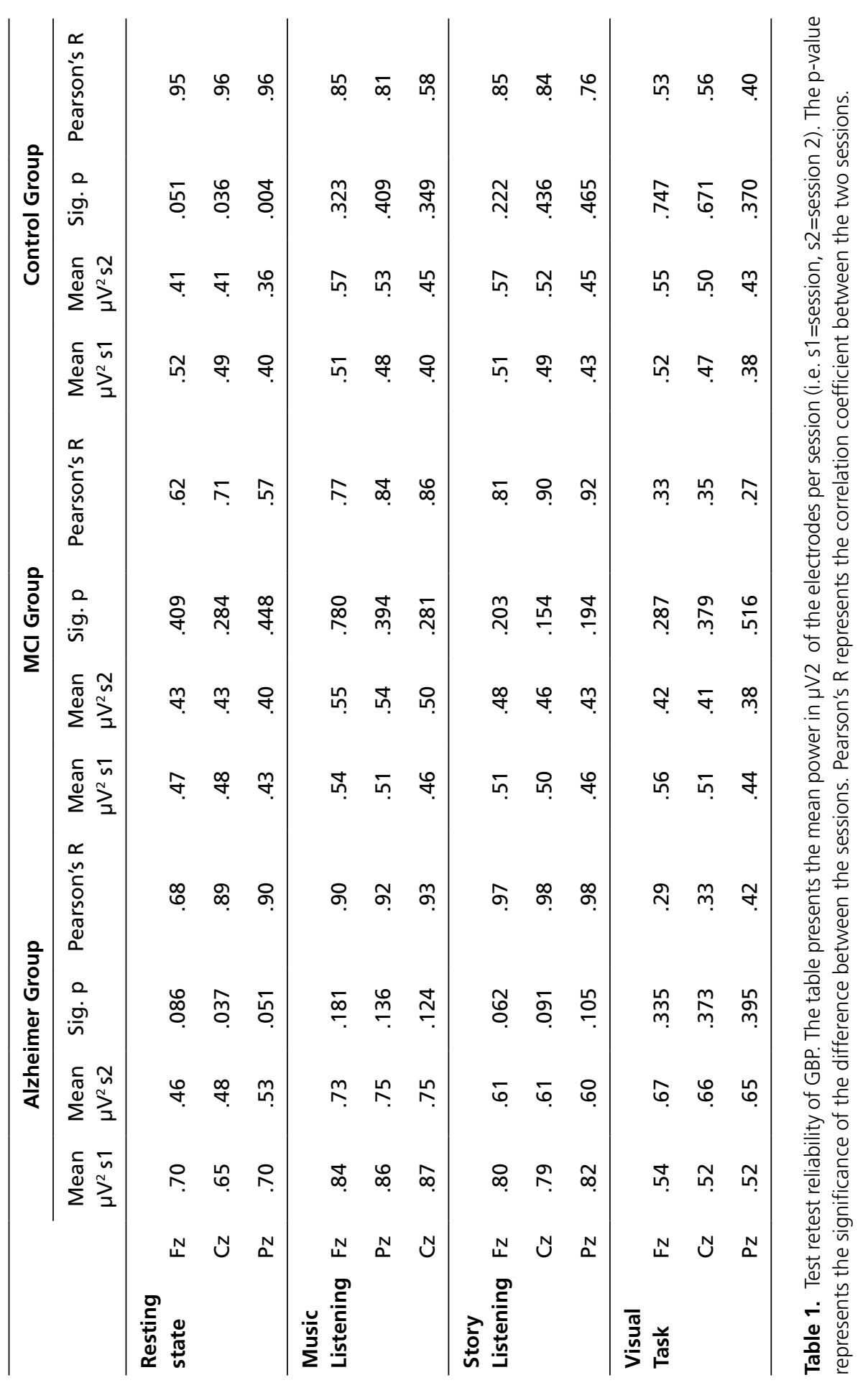


Functional and structural brain markers of Alzheimer's disease | Chapter 3

\section{Resting state}
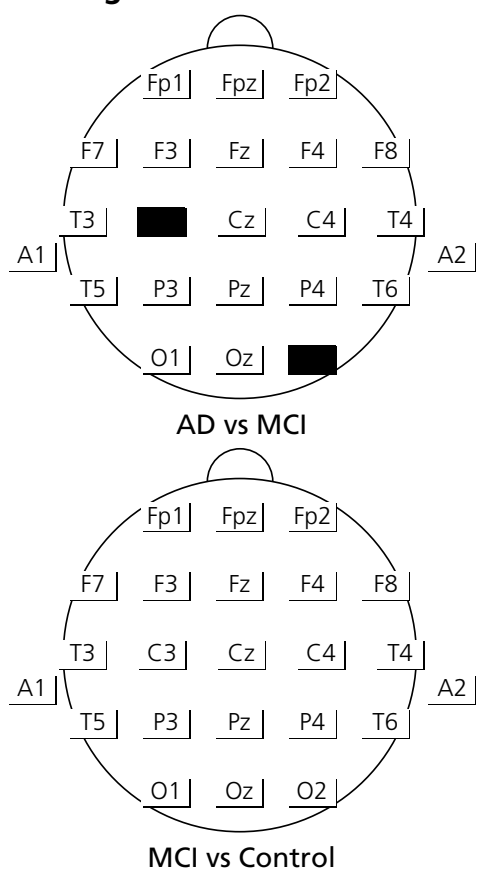

Music listening task

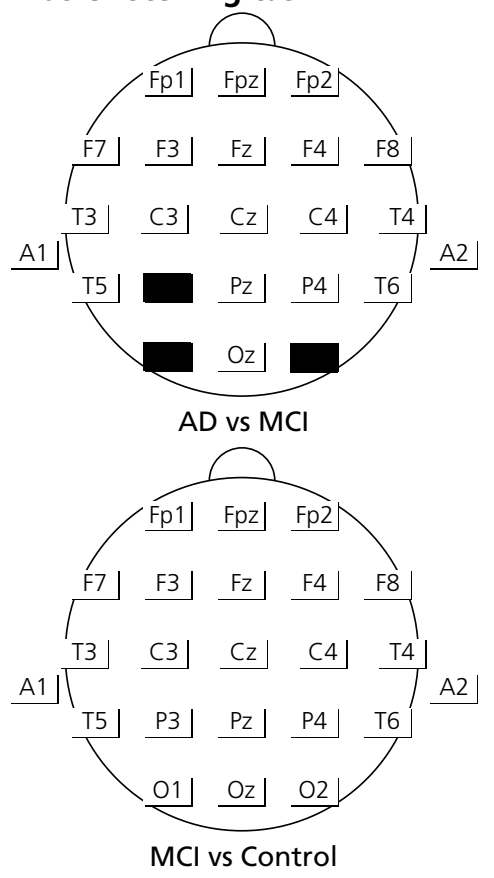

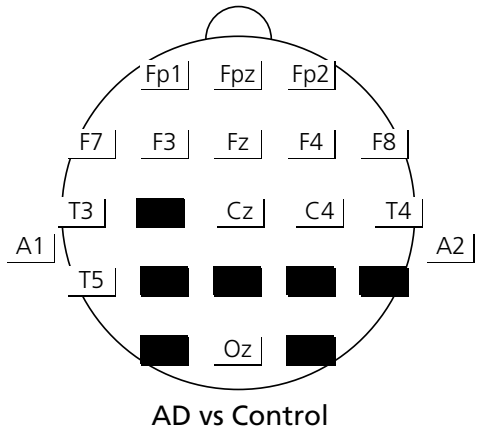

$A D$ vs Control

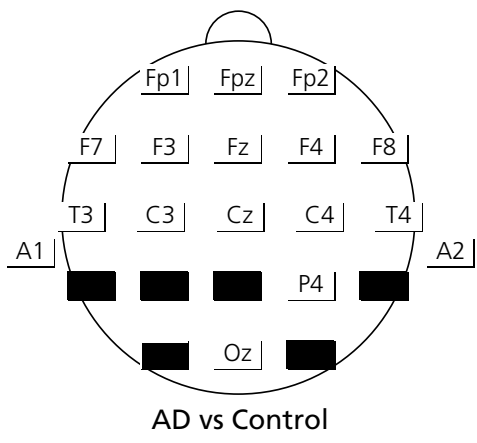




\section{Story listening task}

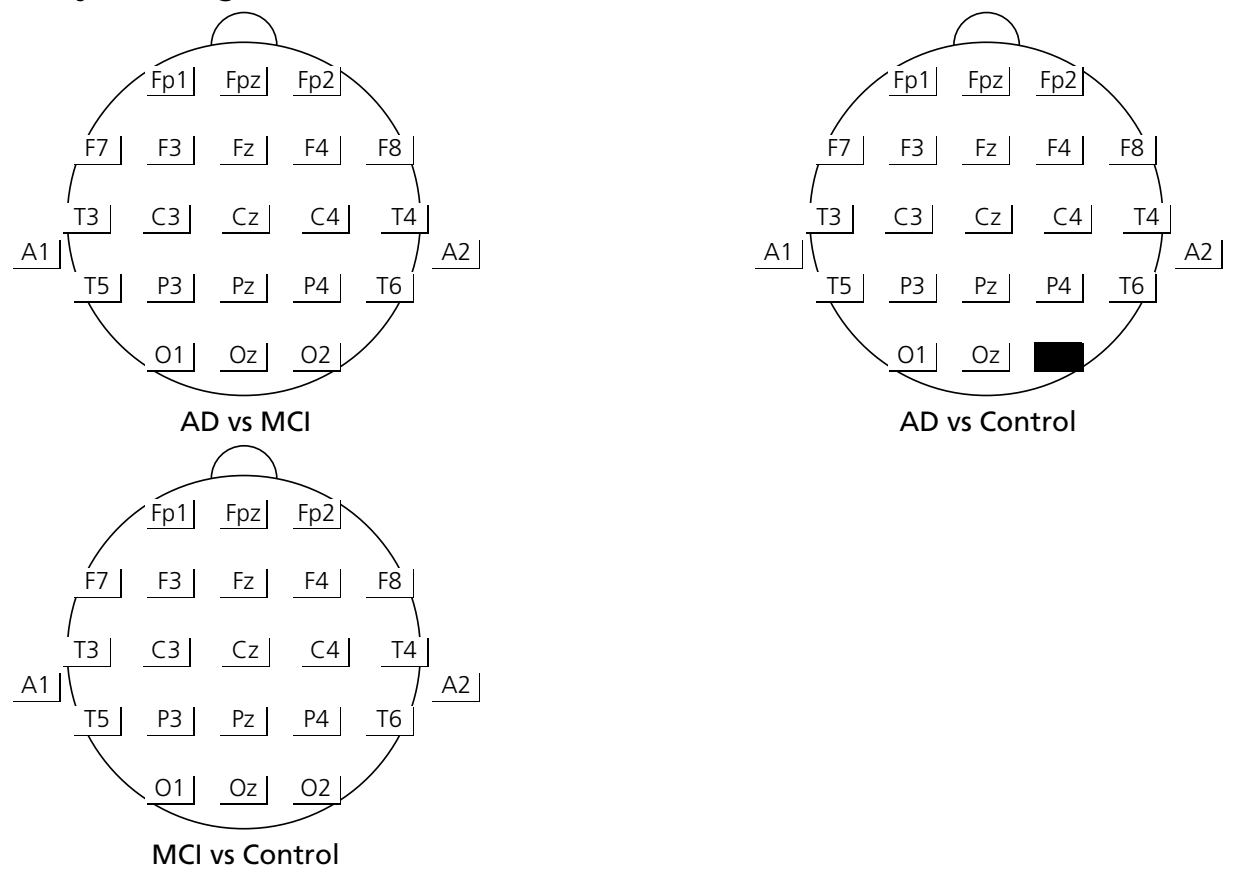

Visual task
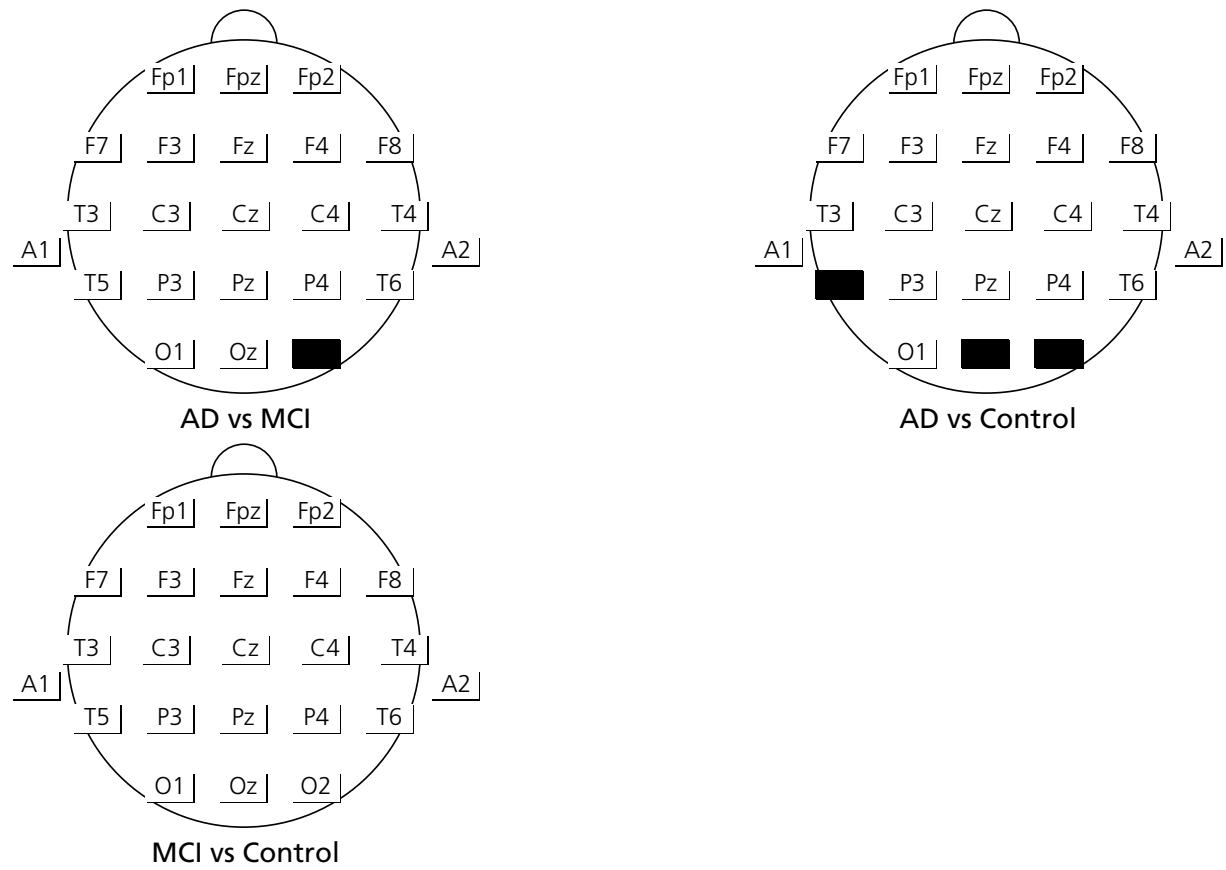

Figure 1: Group comparison of GBP in different paradigms. Solid electrodes represent significantly increased $\mathrm{GBP}$ in $\mathrm{AD}$ compared to $\mathrm{MCl}, \mathrm{AD}$ compared to control and $\mathrm{MCl}$ compared to control. All solid electrodes $(p<.05)$ 


\section{Correlation between GBP and ADAS-Cog}

The correlations between GBP and ADAS-cog are presented in table 2 . The correlations were calculated for the electrodes that significantly separated the patient groups. The results showed a modest but significant correlation between GBP and ADAS-cog in all conditions and all analysed electrodes.

\begin{tabular}{lccc}
\hline & & Pearson's R & Sig. p \\
\hline Resting state & C3 & .38 & .007 \\
& Pz & .39 & .006 \\
& P3 & .39 & .016 \\
& P4 & .38 & .007 \\
& T6 & .39 & .005 \\
& O1 & .47 & .001 \\
\hline Music Listening & O2 & .46 & .001 \\
& Pz & .38 & .005 \\
& P3 & .37 & .007 \\
& T5 & .38 & .005 \\
& T6 & .28 & .005 \\
Story Listening & O1 & .46 & .001 \\
\hline Visual Task & O2 & .47 & .001 \\
\hline & O2 & .33 & .018 \\
\hline
\end{tabular}

Table 2. Correlation between GBP and ADAS-cog. Pearson's $R$ represents the correlation coefficient between GBP and ADAS-cog. The $p$-value represents the significance of the correlation.

\section{Differences between task and resting state condition}

To examine if GBP is altered during task performance we compared GBP during task to resting state.

Figure 2 shows the electrodes with significantly increased GBP in task condition compared to resting state for each group separately together with the overall increase of GBP.

For music listening, GBP increased in the $A D$ at $F z\left(F_{1,1}=4.9, p=.047\right), F 4$ $\left(F_{1,1}=5.6, p=.029\right), F 8\left(F_{1,1}=5.9, p=.031\right), C z\left(F_{1,1}=5.3, p=.041\right)$ and $T 4\left(F_{1,1}=\right.$ $5.2, \mathrm{p}=.042)$. In the $\mathrm{MCl}$ group only $\mathrm{O} 2$ showed a significant increase of GBP 
$\left(F_{1,1}=5.1, p=.047\right)$ compared to resting state. In the control group music listening increased GBP in F4 $\left(F_{1,1}=5.6, p=.029\right), C 4\left(F_{1,1}=5.8, p=.026\right), P 4\left(F_{1,1}=\right.$ 5.4, $\mathrm{p}=.032)$ and $\mathrm{T} 6\left(\mathrm{~F}_{1,1}=7.0, \mathrm{p}=.016\right)$ compared to resting state. When the increase of GBP in music listening was analysed for all subjects irrespective of patient group, a significant difference was shown for Fz, F3, F4, F7, F8, Cz, T4, $\mathrm{T} 5$ and T6. The $\mathrm{F}$ and $\mathrm{p}$ values were in the same order of magnitude as when the groups were analysed separately. The percentages of increase in GBP due to music listening were $155 \%$ in $\mathrm{AD}, 28 \%$ in $\mathrm{MCl}$ and $40 \%$ in the control group. The AD group showed significantly higher increase of GBP due to music listening on $\mathrm{T} 5$ compared to $\mathrm{MCl}$ and controls. There were no other significant differences between the groups in GBP increase due to music listening.

The story listening task showed increased GBP in the AD group for Fp1 $\left(F_{1,1}=4.8, p=.044\right)$ and $F 4\left(F_{1,1}=4.7, p=.049\right)$ compared to resting state. In the $\mathrm{MCl}$ group there was no significant increase or decrease of GBP during story listening compared to resting state. In the control group GBP increased during story listening at $F 3\left(F_{1,1}=5.2, p=.035\right), P 3\left(F_{1,1}=4.9, p=.039\right)$, T5 $\left(F_{1,1}=10.7\right.$, $\mathrm{p}=.004)$ and $\mathrm{T} 6\left(\mathrm{~F}_{1,1}=7.8, \mathrm{p}=.007\right)$ compared to resting state. When the increase of GBP in story listening was analysed for all subjects irrespective of patient group a significant difference was shown for F3, F4, F5, F7, F8, Fp1, T5 andT6. The $F$ and $p$ values were in the same order of magnitude as when the groups were analysed separately. The percentage of increase in GBP due to story listening was $134 \%$ in the $A D, 49 \%$ in $\mathrm{MCl}$ and $68 \%$ in controls. Statistical analysis of story listening related GBP increase showed no significant differences between the groups.

The visual task showed no increased GBP in the AD group compared to resting state. In the $\mathrm{MCl}$ group the visual task showed increased GBP at 01 $\left(F_{1,1}=6.3, p=.016\right)$ compared to resting state. In the control group the visual task showed increased GBP at $F z\left(F_{1,1}=4.4, p=.049\right)$ compared to resting state. When the increase of GBP in the visual task was analysed for all subjects irrespective of patient group a significant difference was shown for F3, F4, F5, F7, F8, Fp1, T5 andT6. The F and $p$ values were in the same order of magnitude as when the groups were analysed separately.

The percentage of increased GBP due to the visual task was $57 \%$ in AD, $26 \%$ in $\mathrm{MCl}$ and $62 \%$ in the control group. Statistical analysis of visual task related GBP increase showed no significant differences between the groups.

The differences between task performance and resting state was highest for the AD and control group. None of the electrodes showed decreased GBP in task performance compared to resting state condition. 
Functional and structural brain markers of Alzheimer's disease | Chapter 3

\section{Music listening task}

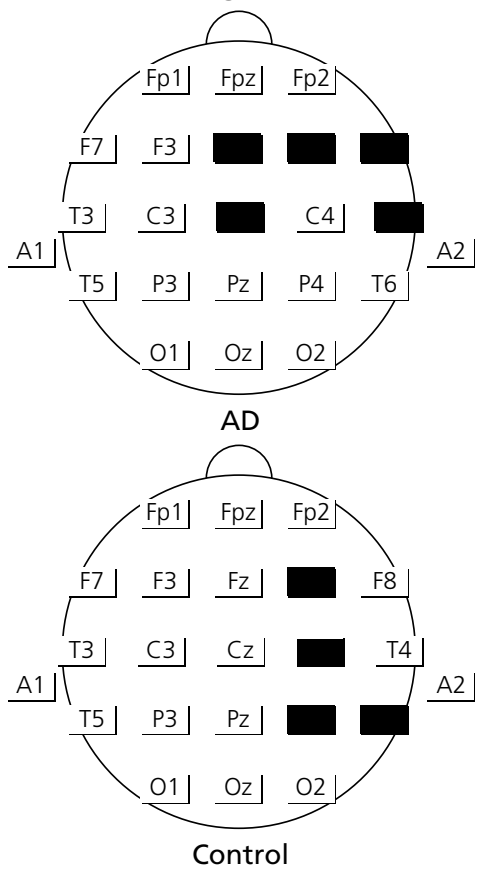

Story listening task

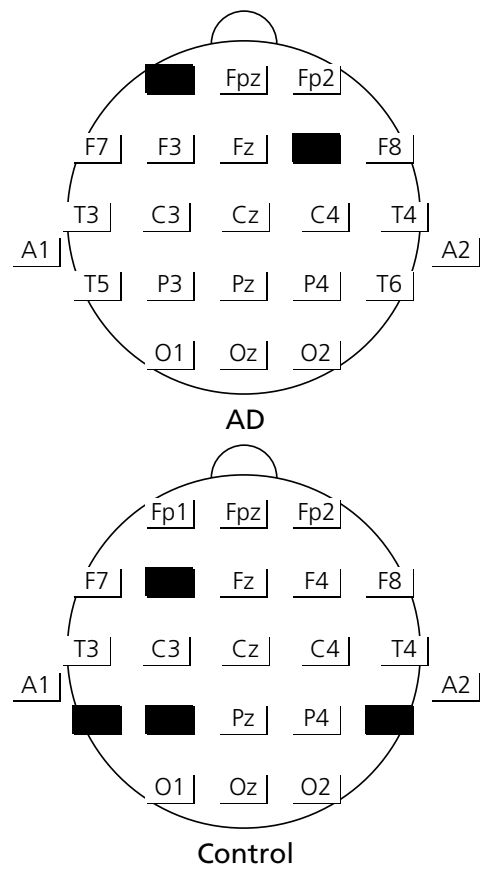

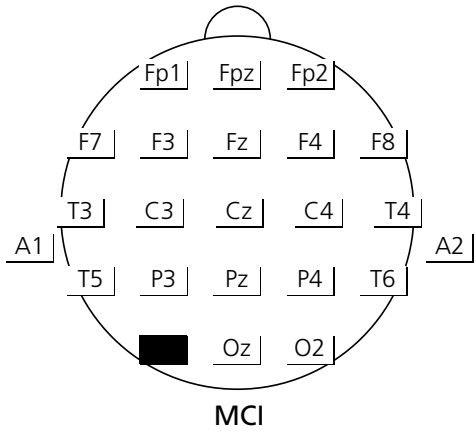
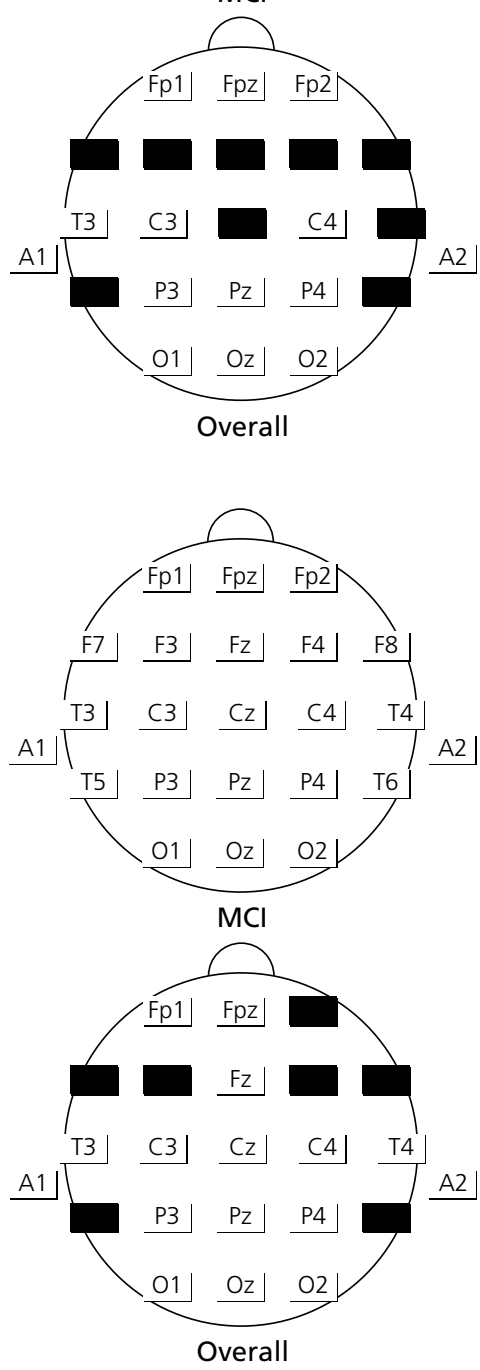


\section{Visual task}
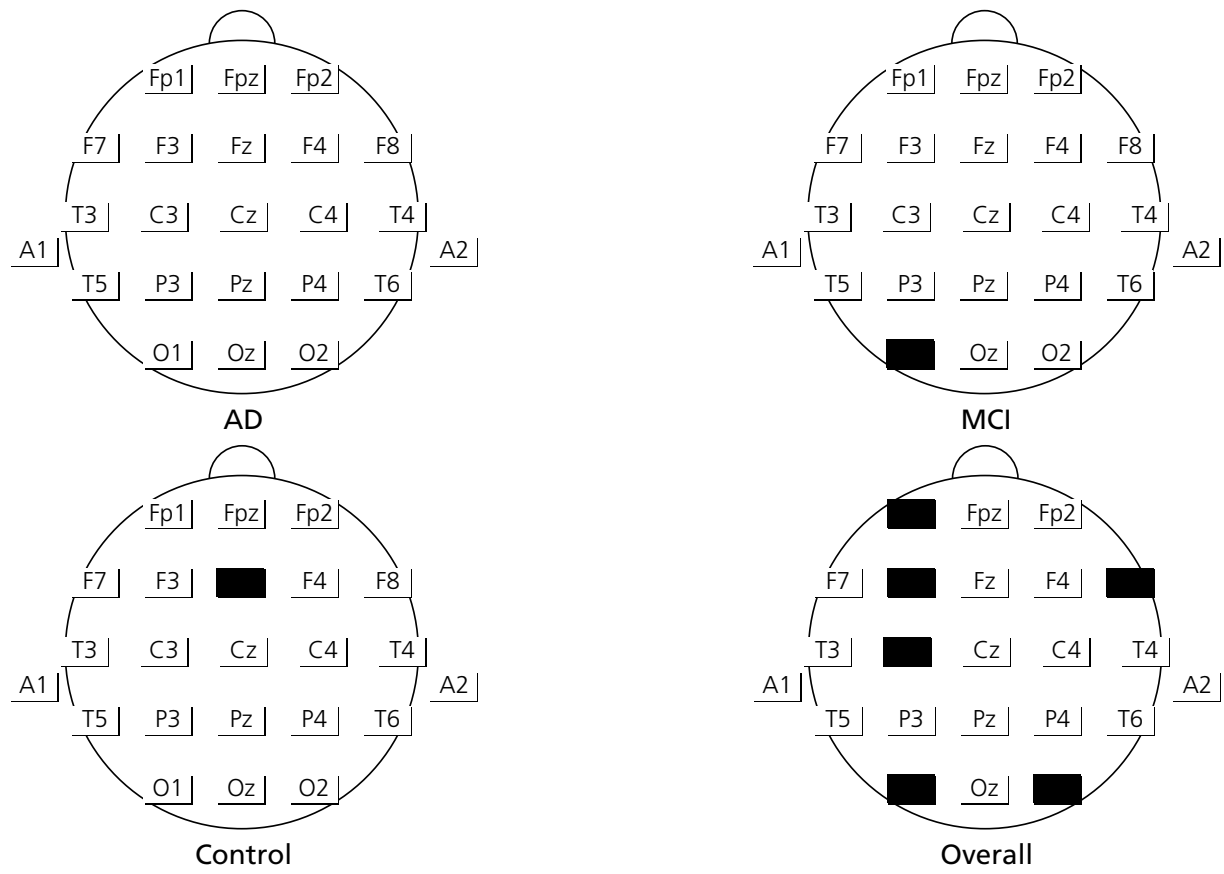

Figure 2: The Solid electrodes represent significantly increased GBP during task performance compared to resting state $(p<.05)$. The results are presented for each group separately. Overall represents significantly increased GBP in task condition for all subjects irrespective of subject group.

\section{Effect of EMG on GBP}

The removal of EMG contaminated epochs resulted in a general decrease of GBP in all conditions (see Table 3). The original differences between the groups remained in the resting state and music listening conditions, except for the $\mathrm{O} 1$ and $\mathrm{O} 2$ electrodes. In the story listening task there were no effects on the

differences between the groups. In the visual task the original differences were no longer significant after EMG correction. The contamination of EMG was found highest in the $\mathrm{O} 1$ and $\mathrm{O} 2$ electrodes. 


\section{Discussion}

Contrary to our hypothesis we found a higher instead of a lower GBP in AD compared to $\mathrm{MCl}$ and controls.

A first step in our analysis was to ensure that differences in GBP between the groups are reproducible and stable, by evaluating test-retest reliability (TRR). An earlier study using a similar design demonstrated a satisfying TRR in young healthy subjects (Frund et al., 2007), but TRR has not been reported in studies with cognitively impaired patients. In the current study, out of 36 comparisons (i.e. three groups, three electrodes and four task conditions) made between the sessions with a paired samples t-test, only three significant differences between sessions were found; one difference in the resting state condition in the AD group, and two in the control group. In spite of these differences, the correlation between the two sessions was high for all electrodes. As shown in Table 1, the resting state GBP is higher in the first session compared to the second. This is most likely related to habituation or diminished arousal in the second session. Another point is that cognitive activity during resting state is an uncontrolled condition that is therefore more difficult to compare between subjects and sessions than under task conditions. The correlation between the sessions in the task conditions was high to very high for all paradigms, except for the visual task. For the latter no satisfying explanation could be found. Overall the TRR results suggest that GBP measures across all stimulus conditions are reproducible in healthy elderly subjects as well as in cognitively impaired patients.

The differences in GBP between the groups are located in the parietal, occipital and posterior temporal regions. These brain areas are also thought to be affected by typical $A D$ neuropathological manifestations (i.e. neuritic plaques and neurofibrillary tangles) especially in the milder stages of $A D$ (Braak and Braak, 1995). The congruence in the location of the GBP changes and $A D$ neuropathology suggests a specific association. Furthermore, we found a significant increase of GBP during task performance compared to resting state, similar to what was shown in healthy subjects by (Fitzgibbon et al., 2004) for all groups. The difference between the groups in task condition was most prominent in the music listening task, which might be related to the complexity of the music stimuli. Music might contain a broader auditory spectrum than the spoken text in story listening and could therefore require more attentional recourses. This increase of GBP during task performance compared to rest was higher in $A D$ than the other groups in music listening and story listening. This task related difference was however only significant 


\begin{tabular}{lcccc}
\hline & & Mean $\mu \mathrm{V}^{2} \mathrm{UC}$ & Mean $\mu \mathrm{V}^{2} \mathrm{C}$ & Sig. $\mathrm{p}$ \\
\hline Resting state & $\mathrm{C} 3$ & .68 & .48 & .048 \\
& $\mathrm{Pz}$ & .45 & .42 & .027 \\
& $\mathrm{P} 3$ & .52 & .46 & .024 \\
& $\mathrm{P} 4$ & .44 & .40 & .031 \\
& $\mathrm{~T} 6$ & .56 & .43 & .026 \\
& $\mathrm{O} 1$ & 1.03 & .60 & .018 \\
\hline Music Listening & $\mathrm{O} 2$ & .86 & .57 & .050 \\
& $\mathrm{Pz}$ & .52 & .47 & .118 \\
& $\mathrm{P3}$ & .59 & .52 & .085 \\
& $\mathrm{~T} 5$ & .95 & .59 & .064 \\
& $\mathrm{~T} 6$ & .76 & .48 & .002 \\
& $\mathrm{O} 1$ & .98 & .58 & .002 \\
\hline Story Listening & $\mathrm{O} 2$ & .86 & .54 & .005 \\
\hline Visual Task & $\mathrm{O} 2$ & .85 & .68 & .000 \\
& $\mathrm{~T} 5$ & .83 & .76 & .085 \\
& $\mathrm{O} 1$ & .88 & .77 & .004 \\
& $\mathrm{O} 2$ & .75 & .75 & .000 \\
\hline
\end{tabular}

Table 3. Effect of EMG on GBP. Paired samples t-test between corrected (C) and uncorrected (UC) EEG. The $p$-value represents the significance of the difference between corrected and uncorrected EEG.

in T5 during music listening, which is probably related to lack of statistical power. The current results suggest that the difference in GBP between the groups was increased by task performance but this needs further substantiation by studies with larger sample sizes.

A difficulty with this type of studies is that gamma band and EMG share the same frequency. A recent study by Whitham et al. (2007) showed convincing evidence for major effects of EMG on GBP. Therefore we retrospectively tried to eliminate this factor. The post-hoc EMG analysis showed a general decrease of GBP in all conditions and for all electrodes. Nevertheless, it was shown that the major results of this study are sustained after reduction of the EMG contamination. The conservative procedure resulted in the removal of suspect EMG epochs, but also diminished the number of epochs with valid gamma band activity. It has to be noted that it is difficult to discriminate low voltage EMG from gamma band activity as they share the same frequency. To the best of our knowledge there are no automated algorithms that remove 
or reduce EMG from the EEG without having an effect on GBP. Therefore we considered the filtering and visual inspection as an adequate method to remove EMG artefacts. The study by Whitham et al. (2007) together with the current results suggest that in induced gamma band studies EMG activity should be taken into account.

A methodologically strong aspect of the current study is that only patients naïve for psychoactive drugs were included in this study. Almost all other studies on gamma band activity in $A D$ included patients on cholinesterase inhibiting drugs, NMDA receptor antagonists or other psychoactive drugs. Several studies have reported that cholinesterase inhibitors have a strong impact on the EEG signal (Kogan et al., 2001; Rodriguez et al., 2002; Rodriguez et al., 2004). This difference between the current study and previous studies might explain why elevated GBP has not been reported earlier. One other study showed enhanced GBP in psychoactive drug naïve $A D$ patients using a $40-\mathrm{Hz}$ steady state response (SSR) paradigm (Osipova et al., 2006).

The finding of increased GBP cannot be explained in a straightforward manner. In contrast to the current findings there are two that showed decreased GBP in $A D$, one in humans and one in animals. The human study showed decreased GBP in a 40-Hz SSR paradigm in AD compared to controls (Ribary et al., 1991). In the animal study GBP was decreased in APP over-expressing mice, measured invitro (Driver et al., 2007). It has to be noted that the methods used in these two studies, fundamentally differ from induced GBP measurements and that therefore these results cannot be compared head to head. It would however for future research be interesting to study the relation between different type of gamma band paradigms in animals and in humans.

Although in the current study relatively simple tasks were used, attentional demands might play an important role in controlled processing during task performance. Increased attention might be required in $A D$ as patients have more difficulty in processing information in an automatic manner compared to the $\mathrm{MCl}$ and control group. The enhanced GBP in AD might therefore reflect an increased level of controlled processing and effortful attention as a functional compensation mechanism in AD. Previous studies have shown that as attentional demand or task difficulty increases, GBP increases (Landau et al., 2007; Tallon-Baudry et al., 2005; Tiitinen et al., 1993). The hypothesis of different attentional demands between the groups is however not substantiated by the current data. In the resting state paradigm no GBP differences between the groups would be expected since there was no selective cognitive activity ongoing. Since the current study showed resting state differences, it cannot be concluded that enhanced GBP in $A D$ is 
primarily the result of a difference in attentional demands. An alternative explanation might be that GBP is continuously enhanced in AD irrespective of sensory stimulation or specific demands on cognitive processing. Decreased resting state gamma band synchronization in AD was earlier demonstrated in the occipito-parietal regions (Koenig et al., 2005; Stam et al., 2002). An fMRI study showed that the posterior cingulate cortex and medial parietal cortex, areas usually activated in resting state condition, and deactivated in task condition are more active in $A D$ subjects than in healthy controls during task performance (Lustig et al., 2003). In the current study GBP was increased in resting state in the same brain areas; the occipito-parietal regions. Another aspect is that induced gamma band oscillations, consist of task-related components as well as task-unrelated components. These task-unrelated oscillations are continuously present and are not lost during averaging of the data. This is in contrast to event-related designs where task-unrelated activity vanishes during pre-processing of the data. These task-unrelated oscillations therefore might have an effect on GBP and could be enhanced in AD. The latter point together with the $\mathrm{fMRI}$ data suggests that the so-called default mode is more active in AD and that this involves the gamma band. Increased default activity might be related to decreased cortical inhibition in AD. Decreased inhibition and hyper excitability of cortical responses in $A D$ has been suggested by a number of studies (Di Lazzaro et al., 2004; Nardone et al., 2006a; Nardone et al., 2006b). Cortical disinhibition and hyper excitability are often associated with dysfunction of glutamate and GABA neurotransmission, which is a known characteristic of AD pathology (Francis, 2005). The role of GABA and glutamate in the regulation of gamma band has been shown in vitro and suggest glutamate as a generator of gamma band oscillations and GABA as an inhibitor (Whittington et al., 2000). How these neurotransmitters regulate gamma band oscillations in the human brain and how this is reflected in scalp EEG is however not clear.

The present study shows a discrepancy with previous studies that showed decreased gamma band synchronization in AD (Koenig et al., 2005; Stam et al., 2002). This decrease in gamma band synchronization can be explained by the loss of long distance corticocortical connections that characterize AD. A study by Stam et al. (2006) showed impaired synchronization of the gamma band especially in the long distance connections. The results from the current study showing increased local GBP in AD suggest that the local networks are preserved. Increased local GBP might therefore reflect a compensation mechanism for decreased long distance connectivity. In the present study, synchronization was not calculated but the higher GBP might represent impaired information integration between different brain areas. Whether there is a relation between GBP and synchroniza- 
tion is dependent on the method used to calculate synchronization (Pereda et al., 2005). For future research it would be interesting to compare synchronization and power data in the same study sample.

In the current study we were not able to show a difference in GBP between the $\mathrm{MCl}$ and control group. Since ADAS-cog separated these two groups significantly it can be concluded that this is not the result of a small difference in cognitive status. The lack of a statistical difference may be related to the fact that $\mathrm{MCl}$ is an ambiguous construct of which the criteria include a heterogeneous population (Visser and Brodaty, 2006). This might explain that there is no difference between the $\mathrm{MCl}$ and control groups. Nevertheless was there a non-significant trend towards a difference between $\mathrm{MCl}$ and controls that was in line with our hypothesis that the GBP in the $\mathrm{MCl}$ group would be in between the GBP of the AD and control group.

In conclusion, our results suggest that GBP is a reproducible measure that is able to differentiate $A D$ patients from $\mathrm{MCl}$ and control subjects. Whether induced GBP measured during ongoing activity is a valid method needs to be substantiated by future research.

\section{Acknowledgements}

This work was financially supported by research agreement CSMD Ref No: 002509 from GlaxoSmithKline, Cambridge, UK. We thank Lieke Smits, MSc for her help with the collection of the data. 


$$
\text { Chapter }
$$





\section{0-Hz steady state response in Alzheimer's disease and mild cognitive impairment}

This chapter was published as

J.A. van Deursen, E.F.P.M Vuurman, V.H.J.M. van Kranen-Mastenbroek F.R.J. Verhey, W.J. Riedel (2009) Neurobiology of aging In press.

\section{Abstract}

The $40-\mathrm{Hz}$ steady state response (SSR) reflects early sensory processing and can be measured with electroencephalography (EEG). The current study compared the $40-\mathrm{Hz}$ SSR in groups consisting of mild Alzheimer's disease patients (AD) $(n=15)$, subjects with mild cognitive impairment $(M C I)(n=20)$ and healthy elderly control subjects $(n=20)$. All participants were naïve for psychoactive drugs. Auditory click trains at a frequency of $40-\mathrm{Hz}$ evoked the $40-\mathrm{Hz}$ SSR. To evaluate test-retest reliability (TRR), subjects underwent a similar assessment one week after the first. The results showed a high TRR and a significant increase of $40-\mathrm{Hz}$ SSR power in the AD group compared to $\mathrm{MCl}$ and controls. The results suggest that $40-\mathrm{Hz}$ SSR might be an interesting candidate marker of disease progression. 


\section{Introduction}

Cortical neural activity is reflected in the electroencephalogram (EEG). Fast neural oscillations measured with EEG and magnetoencephalography (MEG) have been proposed to be an important mechanism in the integration and binding of neural networks in perceptual and cognitive processes (Herrmann et al., 2004; Joliot et al., 1994; Tallon-Baudry and Bertrand, 1999).

A basic method for measuring these fast oscillations is by evoking a $40-\mathrm{Hz}$ steady state response (SSR). The $40-\mathrm{Hz}$ SSR is elicited by auditory stimulation with "click trains" at $40-\mathrm{Hz}$. These very short individual clicks each evoke an event- related response (ERP). The short inter-stimulus intervals in the click trains do not allow the ERP's to return to baseline, resulting in a nearly sinusoidal SSR (Tallon-Baudry and Bertrand, 1999). As a result, the $40-\mathrm{Hz}$ SSR can be considered as a superimposition of middle-latency responses (P50) to each individual click (Galambos et al., 1981). Early gamma band responses such as the $40-\mathrm{Hz}$ SSR are involved in the sensory processes that precede perceptual or attentional processes (Karakas and Basar, 1998).

Impaired sensory processing is one of the hallmarks of Alzheimer's disease (AD). A study using magnetoencephalography (MEG) showed that the $40-\mathrm{Hz}$ SSR in a drug-naïve AD population is enhanced compared to that in healthy controls (Osipova et al., 2006). Osipova et al. related their findings to decreased cortical inhibition in AD. This explanation is substantiated by results from studies using a sensory gating or dual-click paradigm. This paradigm involves the presentation of two consecutive clicks, whereby the P50 amplitude to the second click should be lower. This paradigm is considered a measure for cortical inhibition. Several studies have shown that subjects with AD diverged from the paradigm and exhibited increased P50 amplitude to the second click in AD (Cancelli et al., 2006; Jessen et al., 2001). A study that examined healthy volunteers with a family history of $A D$ also showed a similar divergence in the P50 amplitude (Boutros et al., 1995). This suggests that impaired cortical inhibition may be present very early in the course of the disease. With a view to detecting the disease at an early stage, it is important to identify whether the difference in the $40-\mathrm{Hz}$ rhythm is already present in a well-defined $\mathrm{MCl}$ population.

The current study aims to examine whether differences, similar to the findings of Osipova et al. (2006) are revealed when EEG is used to study AD patients. Furthermore, it sets out to discover whether these differences are already present in subjects with $\mathrm{MCl}$. The current study reports data from a psychoactive drug naïve population. This is an important methodological is- 
sue since several studies showed that pharmacological modulations have a profound effect on the power of the $40-\mathrm{Hz}$ rhythm (Ahveninen et al., 2002; Ahveninen et al., 1999).

To evaluate the robustness and reproducibility of the results, the testretest reliability (TRR) of the $40-\mathrm{Hz}$ SSR paradigm will be evaluated.

We hypothesized that the $40-\mathrm{Hz}$ SSR is enhanced in patients with AD compared to subjects with $\mathrm{MCl}$ and healthy controls. Furthermore, it is to be expected that the SSR in the $\mathrm{MCl}$ group will be higher than in the control group, but lower than in the AD group.

\section{Materials and Methods}

\section{Subjects}

The study included three different groups of subjects:

1. Fifteen psychoactive drug naïve patients with a diagnosis of probable $A D$ according to the NINCDS-ADRDA criteria were included (McKhann et al., 1984). Standard blood workup and neuroimaging (CT or MRI) were carried out, and the diagnosis was supported by abnormal performance on neuropsychological testing.

2. Twenty psychoactive drug naïve patients with a diagnosis of $\mathrm{MCl}$ according to the Petersen criteria were included (Petersen et al., 2001). MCl subjects also received standard blood workup, neuro-imaging and neuropsychological testing. The diagnosis $\mathrm{AD}$ or $\mathrm{MCl}$ was made at a weekly consensus meeting of different specialists.

3. Twenty healthy control subjects were recruited from the Maastricht Aging Study (MAAS), a longitudinal study of the determinants of healthy cognitive aging (van Boxtel et al., 1998). The control subjects were not using any psychoactive medications. Their medical history was screened by a medical health questionnaire. Main exclusion criteria were: a history of stroke, head trauma, and/or any other neurological or psychiatric disorders. Additional exclusion criteria were: severe cardiovascular disease, a Hachinski Ischemic Scale (HIS) (Hachinski, Lassen, \& Marshall, 1974; Rosen, Terry, Fuld, Katzman, \& Peck, 1980) higher than three or a history of substance abuse and/or other serious system diseases (e.g. malignancy, uncontrolled hypertension, neuropathy or seizure disorders).

All $\mathrm{AD}$ and $\mathrm{MCl}$ patients were recruited at the Memory Clinic of the Maastricht University Hospital and were judged competent to give consent by their treating physicians. This study was carried out in accordance with the declaration 
of Helsinki. All participants gave written informed consent prior to the study and in the case of the AD patients a family member also signed the consent form. The local Medical Ethics Committee of the Maastricht University Hospital approved the study.

\section{Experimental procedure}

All subjects took part in two identical recording sessions, temporally spaced apart by one week. On both occasions, the cognitive subscale of the Alzheimer's Disease Assessment Scale (ADAS-cog) (Rosen et al., 1984; Verhey et al., 2004) was assessed prior to EEG acquisition. The Dutch version of the National Adult Reading Test (NART) (Schmand et al., 1998) was assessed to estimate pre-morbid intelligence. The $40-\mathrm{Hz}$ steady-state response was part of a larger study, which further included: induced gamma band measurement; contingent negative variation (CNV); checkerboard task; and an oddball paradigm. Headphones were used to present the auditory stimuli. To evaluate the robustness of the results the test-retest reliability was evaluated. Therefore subjects visited our facilities twice for similar assessments with an interval of one week.

\section{Stimulation}

In the current study there were two conditions; auditory $40-\mathrm{Hz}$ stimulation and resting state. During stimulation condition the subjects were presented with 1-millisecond clicks at a frequency of $40-\mathrm{Hz}$. Headphones presented 80 click trains of 450 ms binaurally. Subjects were instructed to focus on a white crosshair in the centre of a black screen during the $40-\mathrm{Hz}$ stimulation. During the resting state condition, subjects were instructed to keep their eyes open and focus on a white crosshair on a black screen for 90 seconds.

\section{EEG acquisition}

EEG was recorded on a commercially available EEG acquisition system $\left(\mathrm{Nuamps}^{\circledR}\right)$. Electrodes $(\mathrm{AG} / \mathrm{AgCl})$ were positioned following the 10-20 system on a 32-channel electrode cap, using 19 electrodes (Medcat ${ }^{\circledR}$ ). A low-pass filter of $100 \mathrm{~Hz}$ and a high-pass filter of $0,1 \mathrm{~Hz}$ were applied. The sample frequency was $512 \mathrm{~Hz}$ and analogue-digital conversion was 20 bit. Electrode impedance was kept below $5 \mathrm{k} \Omega$. A reference electrode was placed on the right ear lobe. To control for possible vertical eye movements, an electro-oculogram (EOG) electrode was placed one $\mathrm{cm}$ under the midline of the right eye. A ground electrode was placed on the forehead, at Fpz position. EEG's were recorded in a magnetically shielded and sound-attenuated room. During task 
performance subjects were instructed to focus on a white crosshair on a black screen. Subjects were able to see the screen through magnetically shielded glass. Neuroscan ${ }^{\circledR} 4.3$ software was used for EEG recording and analyses.

\section{0-Hz SSR analysis}

EEG analysis was performed off-line. Epochs were recorded in two conditions, during stimulation and during resting state, each with a length of $450 \mathrm{~ms}$. Epochs containing eye-movements, electromyographic activity (EMG), or head motion artefacts were omitted from analysis. Since EMG activity is in the same frequency spectrum as gamma band activity (Whitham et al., 2007), extra effort was taken to remove the EMG activity from the data. This procedure is described in more detail in (van Deursen et al., 2008). As a result the $40-\mathrm{Hz}$ SSR data reported here is completely free of EMG activity. Further pre-processing procedures included; band-pass filtering $(35-45 \mathrm{~Hz}, 48 \mathrm{~dB} / \mathrm{oct})$ and baseline correction. The uncontaminated epochs were transformed from the temporal domain to the frequency domain using Fast-Fourier transformation $(1 \mathrm{~Hz}$ resolution, 512 point block-size, Hanning window $35-45 \mathrm{~Hz}$ ). The mean power at $40-\mathrm{Hz}$ was calculated for each subject and each session.

\section{Statistics}

Significance levels associated with the differences in 40-Hz SSR power between the $A D, M C l$ and control groups were calculated using ANOVA for repeated measures. Electrode position was used as a within-subject variable. Since $40-\mathrm{Hz}$ power was measured in two sessions the mean of these sessions was used in the ANOVA analysis. Post-Hoc Bonferonni correction for multiple comparisons was used. To evaluate the effect of stimulation compared to resting state, ANOVA for repeated measures was used with condition as a within subject variable.

To examine TRR, a paired samples t-test was used to evaluate potential differences between the two sessions for the $\mathrm{Fz}, \mathrm{Cz}$ and $\mathrm{Pz}$ electrode. Furthermore, Pearson's correlation coefficient between the sessions was calculated at $\mathrm{Fz}, \mathrm{Cz}$ and Pz. These analyses were done for each patient group separately.

To examine the relation between $40-\mathrm{Hz}$ power and cognitive performance as measured by ADAS-cog, Pearson's correlation coefficient between these variables was calculated. 


\section{Results}

\section{Subjects' demographics}

Fifteen AD patients were included. They had a mean age of 75.2 (SD 6.9) and a mean MMSE of 20.8 (SD 2.7, range 17-26). Eleven of the AD patients were male. Twenty $\mathrm{MCl}$ patients were included. They had a mean age of 70.6 (SD 7.2) and a mean MMSE of 26.3 (SD 1.6, range 23-29). Twelve of the $\mathrm{MCl}$ patients were male. Twenty healthy control subjects were included. They had a mean age of 69.5 (SD 6.1) and a mean MMSE of 29.3 (SD 0.8, range 28-30). Twelve of the healthy control subjects were male. There was no statistical difference in age $\left(F_{2,52}=2.2, p=.14\right)$ and NART $\left(F_{2,52}=1.7, p=.20\right)$ between the groups

\section{Test-retest reliability}

Table 1 presents the results of the TRR analysis.

The paired samples t-test between the sessions showed a significant difference in the AD group at $\mathrm{Fz}\left(\mathrm{t}_{15,1}: 2.42 ; \mathrm{p}=.032\right)$, and in the $\mathrm{MCl}$ group at $\mathrm{Fz}\left(\mathrm{t}_{19,1}: 3,37\right.$; $p=.004), P z\left(t_{19,1}: 3,44 ; p=.003\right)$ and $C z\left(t_{19,1}: 3,42 ; p=.003\right)$. Despite the significant differences in the t-test the correlation coefficient was high in all groups and in all electrodes.

\section{0-Hz steady state response}

The results of the $40-\mathrm{Hz}$ power analysis during stimulation condition and resting state are presented in figure 1.

During stimulation condition ANOVA analysis showed that the $40-\mathrm{Hz}$ SSR differed between the groups at T5 $\left(F_{52,2}: 3.46 ; p=.034\right)$, T6 $\left(F_{52,2}: 7.20 ; p=.002\right)$ and $\mathrm{O} 2\left(\mathrm{~F}_{52,2}: 4.29 ; \mathrm{p}=.019\right)$. Bonferonni post-hoc analysis for multiple comparisons showed that the $40-\mathrm{Hz}$ power was higher in the $A D$ group compared to controls at T5, T6 and O2. There was a significant difference between AD and $\mathrm{MCl}$ only at $\mathrm{T6}$. There were no significant differences between the $\mathrm{MCl}$ and control group during the stimulation condition.

To ensure that the differences are related to the task and are not due to aspects of the resting state, the effect of stimulation was examined using ANOVA for repeated measures. Results showed a significant increase in $40-\mathrm{Hz}$ SSR power during stimulation condition compared to resting state condition at T5 $\left(\mathrm{F}_{52,2}: 32.00 ; \mathrm{p}<.000\right), \mathrm{T} 6\left(\mathrm{~F}_{52,2}: 30.61 ; \mathrm{p}<.000\right)$ and $\mathrm{O} 2\left(\mathrm{~F}_{52,2}: 23.08 ; \mathrm{p}<.000\right)$.

The $40-\mathrm{Hz}$ power showed a moderate but significant correlation with cognitive performance as measured with ADAS-cog at T5 $(r=.43, p=.019)$ and $\mathrm{T} 6(\mathrm{r}=.38, \mathrm{p}=.028)$. The correlation between $40-\mathrm{Hz}$ power at $\mathrm{O} 2$ and ADAS-cog was low and not significant $(r=.19, p=.064)$. 


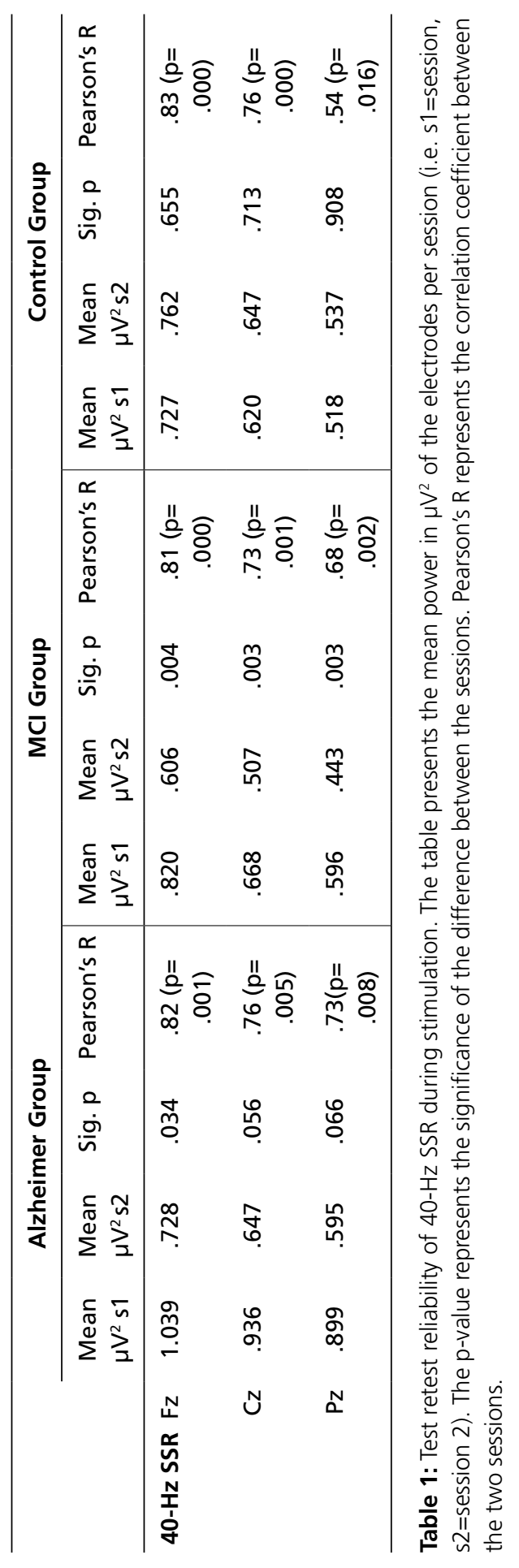




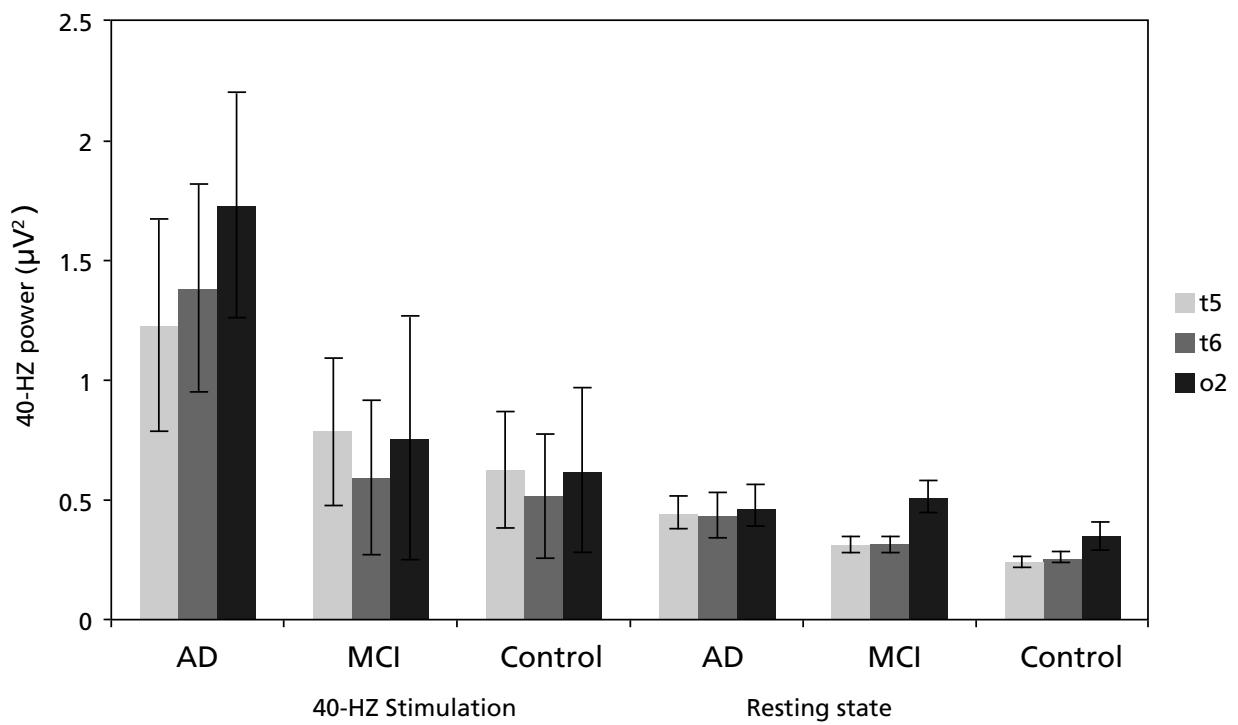

Figure 1. The mean \pm SEM 40-Hz SSR power for each group in stimulation condition (left) and resting state condition (right). In stimulation condition $40-\mathrm{Hz}$ SSR power was higher in the AD group compared to controls at T5, T6 and O2. At T6 there was a difference between $\mathrm{AD}$ and $\mathrm{MCI}$. During stimulation condition $40-\mathrm{Hz}$ SSR power was significantly higher than in resting state.

\section{Discussion}

The current study showed almost a universally high TRR and a significant difference in $40-\mathrm{Hz}$ SSR power between the patient groups. The correlations between the sessions ranged from 0.68 to 0.83 , which is high, only the Pz electrode in the control group showed a moderate correlation (i.e. 0.54). These high correlations suggest that the $40-\mathrm{Hz}$ SSR can reliably be assessed in a cognitively impaired population.

$40-\mathrm{HzHz}$ SSR power was higher the AD group compared to $\mathrm{MCl}$ and control groups. Additionally, we also discovered a difference between the $A D$ and $\mathrm{MCl}$ group. The difference between the $A D$ and control group is in line with a previous MEG study by Osipova et al. (2006). A difference between AD and $\mathrm{MCl}$ with regard to $40-\mathrm{Hz}$ SSR power has not been reported before this study. The absence of a difference between $\mathrm{MCl}$ and controls might be related to the heterogeneous nature of the $\mathrm{MCl}$ concept (Visser and Brodaty, 2006). $\mathrm{MCl}$ not only includes patients in the prodromal stage of $A D$, but also patients with other causes for cognitive impairment. Had we confined ourselves to amnestic $\mathrm{MCl}$ patients, who are more prone to progress to AD (Petersen, 2004), the dif- 
ference between $\mathrm{MCl}$ and controls would probably have been larger. Despite the absence of a significant difference, the $40-\mathrm{Hz}$ SSR power in the $\mathrm{MCl}$ group is in between that the $A D$ and the control group. This finding together with the significant correlation between $40-\mathrm{Hz}$ SSR power and cognitive performance suggests that the $40-\mathrm{Hz}$ SSR power increases as the severity of the disease increases.

In EEG studies of an elderly population differences in age can be a possible confounder of the results. Although the results show no significant differences between the groups with regard to age and pre-morbid intelligence, we performed a post-hoc analysis to ensure that these parameters did not bias the results. Age and pre-morbid intelligence were entered as covariates in the GLM model and proved to have no effect on the group results. Another possible confounder in the $40-\mathrm{Hz}$ SSR measurements is the contamination with EMG. In a previous study we showed that when an extra EMG reduction procedure is performed, gamma band $(30-100 \mathrm{~Hz})$ power is reduced (van Deursen et al., 2008). Therefore in the current study, the same procedure was included in the preprocessing steps, which removed this possible confounder from our data. A methodologically strong aspect of the current study was that only psychoactive drug naïve patients were included. Most drugs used in AD affect the cholinergic nervous system. Since acetylcholine seems to be involved in the regulation of the $40-\mathrm{Hz}$ SSR, these types of drugs will bias the results when medicated AD patients are included. The cholinergic involvement in the regulations of $40-\mathrm{Hz}$ SSR has been suggested by a study that showed that scopolamine, which is a muscarine antagonist that is often used as a pharmacological model for $A D$, increased $40-\mathrm{Hz}$ power in healthy young and elderly subjects (Ahveninen et al., 2002; Ahveninen et al., 1999).

The location of the group differences indicates that the main difference was found in the auditory cortex, which is in agreement with a previous MEG study (Osipova et al., 2006). The auditory cortex has been proposed as the main generator of the 40-Hz rhythm (Gutschalk et al., 1999) although thalamicocortical circuits also seem to play a role (Ribary et al., 1991). The temporal cortex is one of the first areas to be affected by the neurodegenerative processes in AD (Braak and Braak, 1995). Therefore, the difference between the groups is most probably related to changes in the $40-\mathrm{Hz}$ generators that are located in the temporal cortex.

Increased $40-\mathrm{Hz}$ SSR power in the auditory cortex of AD patients has previously been associated with decreased inhibition of the superimposed middlelatency auditory peaks that are related to sensory processing (Osipova et al., 2006). Dual-click sensory gating paradigms showed that in AD the second P50 
peak is increased compared to healthy controls, which suggests impaired cortical inhibition (Cancelli et al., 2006; Jessen et al., 2001). The current results confirm these previous findings.

Cortical disinhibition in AD can be associated with dysfunction of the neurotransmitters gamma-aminobutyric acid (GABA) and glutamate (Jacob et al., 2008). There is variable evidence for dysfunction of GABA and glutamate in AD (Francis, 2003; Garcia-Alloza et al., 2006; Lanctot et al., 2004; Lowe et al., 1988). GABA seems to play an important role in the regulation of the $40-\mathrm{Hz}$ SSR, as administration of the GABA agonist temazepam attenuates the $40-\mathrm{Hz}$ SSR (Jaaskelainen et al., 1999). The role of acetylcholine, GABA and glutamate in the regulation of the $40-\mathrm{Hz}$ rhythm is however not clearly understood and will need more attention in future research.

\section{Conclusion}

In sum, the current study showed that the 40-Hz SSR power is higher in patients with $\mathrm{AD}$ compared to $\mathrm{MCl}$ subjects and healthy controls. The high TRR together with the correlation with cognitive performance suggest that the $40-\mathrm{Hz}$ SSR can reliably be used to measure disease progression.

\section{Acknowledgements}

This work was financially supported by research agreement CSMD Ref No: $\underline{002509}$ from GlaxoSmithKline, Cambridge, UK. We thank Lieke Smits, MSc for her help with the collection of the data. 


$$
\text { Chapter }
$$





\title{
Response speed, contingent negative variation and P300 in Alzheimer's disease and $\mathrm{MCl}$
}

\author{
This chapter was published as
}

J.A. van Deursen, E.F.P.M. Vuurman, L.L. Smits, F.R.J. Verhey, W.J. Riedel (2008) Brain and Cognition In press

\begin{abstract}
Background: Decreased speed of information processing is a hallmark of Alzheimer's disease $(A D)$ and mild cognitive impairment (MCI). Recent studies suggest that response speed (RS) measures are very sensitive indicators of changes in longitudinal follow-up studies. Insight into the psycho-physiological underpinnings of slowed RS can be provided by measuring the associated event-related potentials (ERP).

Aims: The current study aims to investigate the relation between RS and its psychophysiological correlates in $\mathrm{AD}$ and $\mathrm{MCl}$.

Methods: Fifteen psychoactive drug-naïve $A D$ patients, twenty $\mathrm{MCl}$ patients and twenty age-matched, healthy control subjects participated. Response speed was measured during a simple (SRT) and choice reaction time task (CRT). An oddball and contingent negative variation (CNV) paradigm were used to elicit ERP. To evaluate test-retest reliability (TRR), subjects underwent a similar assessment one week after the first.
\end{abstract}


Results: The SRT and CRT distinguished the patient groups significantly. The P300 amplitude and latency also distinguished the groups and showed a significant correlation with response speed. The CNV amplitude did not reveal a significant difference between groups and also showed a low TRR.

The TRR of the SRT, CRT and P300 amplitude and latency in general was moderate to high. The current study suggests that response speed measures on a behavioural and psycho-physiological level deserve attention as a possible marker in the diagnosis and follow-up of AD.

\section{Introduction}

Decreased speed of information processing is one of the hallmarks of cognitive aging. Several studies have shown an age-related increase of reaction times (RT) in a variety of cognitive tasks (Salthouse, 2000). Typically response speed (RS) is measured during tasks measuring simple reaction time (SRT), choice reaction time (CRT), rapid visual information processing, speed of scanning working memory, or similar processes. The SRT primarily reflects sensory encoding and psychomotor speed, whereas the CRT captures an additional decision-making component (Gordon and Carson, 1990). Furthermore, the speed of response is associated with focussed and sustained attention (Salthouse, 1996; Salthouse, 2000; Verhaeghen and De Meersman, 1998). Augmented slowing of RS is a characteristic of Alzheimer's disease and distinguishes patients from elderly controls (Gordon and Carson, 1990; Levinoff et al., 2005; Storandt and Beaudreau, 2004).

The clinical diagnosis of $A D$ is based on the outcome of an extensive medical and neuropsychological evaluation. However, when monitoring the progression of the disease, or the evaluation of treatment intervention, the Alzheimer's Disease Assessment Scale - Cognitive Subscale (ADAS-cog) is routinely used (Rosen et al., 1984; Verhey et al., 2004). The ADAS-cog measures a variety of cognitive functions but does not include any measure of attention or RS (Wesnes, 2008). A 12-week follow-up study on the effects of Galantmine on cognitive performance in $A D$, showed that RS measurements were more sensitive indicators of changes than the ADAS-cog (Caramelli et al., 2004). A recent neuroimaging study showed that adding attentional or speed measures to ADAS-cog, improves this instrument's sensitivity as a means to predict white matter changes in an elderly population (Ylikoski et al., 2007). These findings suggest that RT measures could also improve the sensitivity of the ADAS-cog in the behavioural domain. 
Response speed is the behavioural endpoint of a cascade of neural processes. These processes can be unravelled by measuring psycho-physiological brain activity with the aid of event-related potentials (ERP). The ERP typically associated with RS and stimulus classification are contingent negative variation (CNV) and P300. Measuring these ERP simultaneously with RS can provide more insight into the neural underpinnings of slowed RS in AD. The CNV is a slow negative potential that precedes a response to an anticipated stimulus. The typical CNV paradigm consists of a warning stimulus (S1) followed by the imperative (S2) stimulus four seconds later (Walter et al., 1964). The amplitude of the CNV complex is believed to reflect attention, expectancy, intention to respond and motor preparation. The amplitude of the CNV component seems to be related to RS: larger CNV amplitudes precede shorter reaction times (Brunia and Vingerhoets, 1980; Brunia and Vingerhoets, 1981; Haagh and Brunia, 1985). Early studies showed decreased CNV amplitudes in Alzheimer's disease and $\mathrm{MCl}$ but it has to be noted that very small sample sizes were used ( $\mathrm{O}$ 'Conner, 1980; Zappoli et al., 1991). Therefore, a comparison with larger sample sizes of CNV activity in these patient groups is necessary. The P300 is elicited in response to deviant stimuli in simple auditory or visual discrimination tasks. The amplitude of the P300 is considered as the manifestation of brain activity that reflects attention to incoming stimulus information when representations are updated (Polich, 2007). In general, passive stimulus processing produces smaller P300 amplitudes than active stimulus processing. The P300 latency is considered as stimulus classification speed and is sensitive to task processing demands and cognitive abilities (Polich, 2007). Previous studies have reported decreased P300 amplitudes and increased latencies in AD (Boutros, Torello, Burns, Wu, \& Nasrallah, 1995; Golob \& Starr, 2000; Ito, Yamao, Fukuda, Mimori, \& Nakamura, 1990; Patterson, Michalewski, \& Starr, 1988; Polich, Ladish, \& Bloom, 1990; Szelies, Mielke, Grond, \& Heiss, 1995) (Boutros et al., 1995; Golob and Starr, 2000; Ito et al., 1990; Patterson et al., 1988; Polich et al., 1990; Szelies et al., 1995). Despite these consistent findings, there are also studies that did not show a difference between AD and healthy controls on P300 amplitude and latency (Verleger et al., 1992). Other studies showed a difference between $A D$ and healthy controls for P300 amplitude only (Duffy et al., 1984) or on P300 latency only (Ito et al., 1990). For an extensive review, see (Polich and Herbst, 2000). There is some evidence that suggests that a larger P300 amplitude and slower latency is related to faster responses. However, this relation has only been shown in healthy subjects and in MCl but not yet in AD (Dimoska et al., 2006; Williams et al., 1991). Most ERP studies in AD have been performed with patients on cholinesteraseinhibiting drug treatment. These drugs have a profound effect on ERP ampli- 
Functional and structural brain markers of Alzheimer's disease | Chapter 5

tudes and latencies (Katada et al., 2003; Werber et al., 2003). It is therefore of importance to study the relationship between RS and ERP in a drug-naïve population.

A relatively underexposed aspect of ERP recordings is their reliability when it comes to monitoring cognitively impaired patients. If RS measures or ERP are to be used as markers in the diagnosis and follow-up of $A D$ and $\mathrm{MCl}$ in the future, it is important to test their reliability. Therefore, the TRR of the RS tasks and the ERP have been a point of focus in the current study. The current study examines the relation between RS and the psycho-physiological correlate in cases of $A D$ and in cases of $\mathrm{MCl}$, as well as in healthy elderly control subjects.

\section{Materials and Methods}

\section{Subjects}

The study involved three different groups of subjects. The first group consisted of fifteen patients who were psychoactive drug naïve and diagnosed with probable AD according to the NINCDS-ADRDA criteria (McKhann et al., 1984). Standard blood workup and neuro-imaging (CT of MRI) were carried out on this group, and the diagnosis was supported by abnormal performance on neuropsychological testing.

The second group consisted of twenty patients who were psychoactive drug naïve and diagnosed with $\mathrm{MCl}$ according to the Petersen criteria (Petersen et al., 2001). These $\mathrm{MCl}$ subjects also received standard blood workup, neuro-imaging and neuropsychological testing. The diagnosis $A D$ or $\mathrm{MCl}$ was made during a weekly consensus meeting of different specialists. All $\mathrm{MCI}$ patients showed objective cognitive disturbances and were divided in the following sub-classifications: Five of the $\mathrm{MCl}$ patients had single domain amnestic $\mathrm{MCl}$; eight patients had multiple domain amnestic $\mathrm{MCl}$; two patients had single domain non-amnestic $\mathrm{MCl}$; and five had multiple domain nonamnestic $\mathrm{MCl}$ (Petersen, 2004).

The third group consisted of twenty healthy control subjects who were recruited from the Maastricht Aging Study (MAAS), a longitudinal study of the determinants of healthy cognitive aging (van Boxtel et al., 1998). None of the healthy controls used psychoactive medication. Their medical history was screened by a medical health questionnaire. The cognitive status of the control subjects was screened with the Mini Mental State Examination (MMSE), using a cut-off score of $\geq 28$ (Folstein et al., 1975c). The test scores on the ADAS-cog were also used to ensure that the control group had normal cognitive abilities. 
Main exclusion criteria were: a history of stroke, head trauma, and/or any other neurological or psychiatric disorders. Additional exclusion criteria were: severe cardiovascular disease, a Hachinski Ischemic Scale (HIS) (Hachinski et al., 1974; Rosen et al., 1980) higher than three and/or a history of substance abuse and/or other serious system diseases (e.g. malignancy, uncontrolled hypertension, neuropathy or seizure disorders).

All $\mathrm{AD}$ and $\mathrm{MCl}$ patients were recruited at the Memory Clinic of University Hospital Maastricht and were judged competent to give consent by their treating physicians. All participants gave written informed consent prior to the study; in the case of $A D$ patients a family member also signed the consent form. The local Medical Ethics Committee of the University Hospital Maastricht approved the study.

\section{Experimental procedure}

All subjects took part in two identical recording sessions, temporally spaced apart by one week. On both occasions the cognitive subscale of the Alzheimer's Disease Assessment Scale (ADAS-cog) (Rosen et al., 1984; Verhey et al., 2004) was assessed prior to EEG acquisition. The Dutch version of the National Adult Reading Test (NART) (Schmand et al., 1991) was performed to estimate pre-morbid intelligence. A simple reaction time task (SRT) and a choice reaction time task (CRT) were performed to measure reaction time speed. After the assessment of NART, ADAS-cog and the response speed tasks, there was a break of 30 minutes in which the participants could rest while the EEG cap was attached. The CNV and P300 EEG data were acquired in the first fifteen minutes of the EEG test session to prevent effects of fatigue on the results. The two electrophysiological outcome measures i.e. CNV and P300 were part of a larger study, which further included: three gamma band paradigms, $40-\mathrm{Hz}$ steady state response and a visual checkerboard task. The order of the administration of the tasks was similar for each patient and on both test sessions.

\section{Behavioural paradigms}

Simple and Choice reaction time tasks

The simple reaction time task (SRT) was presented in four runs of 12 trials each. In each trial of the SRT a white square $(4 \times 4 \mathrm{~cm})$ on a black background was followed by a red square $(4 \times 4 \mathrm{~cm})$. Subjects were instructed to push the response button as fast as possible when the white square turned red. After the response, the red square turned white again until the next trial. The interval between the trials varied randomly between two and six seconds. Between the runs there was a break of one minute. 
The choice reaction time task (CRT) was presented in four runs of 12 trials. In each trial two whites squares $(4 \times 4 \mathrm{~cm})$, one on the left side and one on the right side of the screen, were presented on a black background. When one of the two squares turned red, the subject had to push the congruent response button, left or right. After the response the red square turned white again. The interval between the trials varied randomly between two and six seconds.

The visual stimuli were presented on a 16 -inch computer screen. The responses were given on a standard five-options response box. The SRT and CRT were practiced for three minutes prior to measurement during each visit, in order to prevent learning effects and to familiarize the subjects with the tasks.

\section{CNV paradigm}

The visual CNV paradigm consisted of a warning stimulus in the form of a big red dot $(\varnothing 12 \mathrm{~cm})$, followed by the imperative stimulus in the form of a big green dot $(\varnothing 12 \mathrm{~cm})$. A total of 36 trials were administered, the inter-stimulus interval (ISI) was two seconds and the inter-trial interval (ITI) varied between five and ten seconds. The subjects were instructed to respond as fast as possible when the imperative stimulus appeared. The task was practised for three minutes prior to each recording session to prevent learning effects and to familiarize the subjects with the task. To prevent differences between the groups regarding practice intensity, the practice trails were similar for all subjects.

\section{Oddball paradigm}

A simple auditory two-tone discrimination or 'oddball' paradigm was used to elicit ERP responses. Thirty-two target tones $(2000 \mathrm{~Hz}, 80 \mathrm{~dB}, 100 \mathrm{~ms})$ were pseudo-randomly distributed within a stream of non-target tones $(1000 \mathrm{~Hz}, 80$ $\mathrm{dB}, 100 \mathrm{~ms}$ ). A fixed inter-stimulus interval of two seconds was used. Target and non-target stimuli appeared with a probability of $15 \%$ and $85 \%$ respectively. Subjects were requested to press a hand-held button when they detected a target stimulus. They were instructed to respond accurately without any emphasis on a speedy response in order to limit the possible contamination of the P300 with motor potentials. The task was practised for three minutes prior to each recording session to prevent learning effects and to familiarize the subjects with the task.

\section{EEG acquisition}

The EEG was recorded in a magnetically shielded and sound-attenuated room. The visual stimuli were presented on a flat LCD screen located outside the room. Subjects were able to see the screen through magnetically shielded glass. The 
auditory stimuli were presented from headphones placed on both ears. EEG was recorded on a commercially available EEG acquisition system (Nuamps ${ }^{\circledR}$ ). Electrodes $(\mathrm{Ag} / \mathrm{AgCl})$ were positioned following the 10-20 system on a 32-channel electrode cap, using 19 electrodes (Medcat ${ }^{\circledR}$ ). For the CNV recordings, a low-pass filter of $100 \mathrm{~Hz}$ and a high-pass filter of $0,01 \mathrm{~Hz}$ were applied. For the oddball paradigm a low-pass filter of $100 \mathrm{~Hz}$ and a high-pass filter of $0,1 \mathrm{~Hz}$ were applied. The sample frequency was $512 \mathrm{~Hz}$ and analogue-digital conversion was 20 bit. Electrode impedance was kept below $5 \mathrm{k} \Omega$. A reference electrode was placed on the right ear lobe. To control for possible vertical eye movements, an electro-oculogram (EOG) electrode was placed one centimeter under the midline of the right eye. A ground electrode was placed on the forehead, at Fpz position. Neuroscan ${ }^{\circledR} 4.3$ software was used for EEG recording and analyses.

\section{ERP data analyses \\ CNV}

The interval of one second prior to the imperative stimulus was analysed for CNV. Pre-processing procedures included ocular artefact reduction, low-pass filtering $(0-30 \mathrm{~Hz}, 12 \mathrm{~dB} / \mathrm{oct})$, baseline correction and averaging of the data. The interval between 900-1000 milliseconds after the warning stimulus was used for the baseline correction procedure. The peak amplitude of the epochs was calculated for each subject. The reaction times were calculated relative to onset of the imperative stimulus. Mean reaction times over the 36 trials were calculated; trials with reaction times faster than $60 \mathrm{~ms}$ and slower than $600 \mathrm{~ms}$ were omitted from analysis.

\section{Oddball paradigm}

The pre-processing steps of the oddball data included high-pass filtering (> $1 \mathrm{~Hz} 12 \mathrm{~dB} /$ oct), ocular artefact reduction, low-pass filtering $(<30 \mathrm{~Hz}, 12 \mathrm{~dB}$ / oct), baseline correction and averaging. Peak latencies were determined relative to the stimulus onset. The ERP epochs for the target and non-target tones were analysed separately. Amplitudes of the P300 and N200 were defined relative to a baseline period, $100 \mathrm{~ms}$ prior to stimulus onset. An automated peak-picking procedure was used to determine peak amplitudes and latencies. N200 was defined as the minimum point between $200-250$ ms post stimulus. The P300 was defined as the maximum point between $300-600 \mathrm{~ms}$ post stimulus. There were no reaction times registered in the oddball paradigm. 


\section{Statistical analysis}

The primary endpoints in the current study were CNV amplitude, P300 amplitude and latency and reaction times. Statistical analysis was done with SPSS for Mac (version 16.0). Significance levels associated with the differences in ERP amplitude and latency between the $A D, M C l$ and control groups were calculated for $\mathrm{Fz}, \mathrm{Cz}$ and $\mathrm{Pz}$ using ANOVA. Post-Hoc Bonferonni correction for multiple comparisons was used. For the ANOVA analysis, the mean ERP amplitude and latency over the two sessions were calculated for each subject and each electrode.

Reaction times were averaged for each subject and each session. ANOVA with post-hoc Bonferonni correction was used to calculate between group differences.

To examine test-retest reliability of the ERP, a paired samples t-test was used to evaluate potential differences between the two sessions for the $\mathrm{Fz}, \mathrm{Cz}$ and $\mathrm{Pz}$ electrode. Furthermore, Pearson's correlation coefficient between the sessions was calculated at $\mathrm{Fz}, \mathrm{Cz}$ and $\mathrm{Pz}$. These analyses were done for each patient group separately. The same statistics were applied to evaluate the TRR of the reaction times. To evaluate the relation between the outcome measures and cognitive performance on ADAS-cog, Pearson's correlation coefficient between the outcome measures and ADAS-cog was calculated.

\section{Results}

\section{Subjects' demographics}

The fifteen AD patients had a mean age of 75.2 (SD 6.9) and a mean score on the Mini Mental State Examination (MMSE) of 20.8 (SD 2.7, range 17-26). Eleven of the $A D$ patients were male. The twenty $M C l$ patients included had a mean age of 70.6 (SD 7.2) and a mean MMSE of 26.3 (SD 1.6, range 23-29). Twelve of the $\mathrm{MCl}$ patients were male. The twenty healthy control subjects included had a mean age of 69.5 (SD 6.1) and a mean MMSE of 29.3 (SD 0.8, range 28-30). Twelve of the healthy control subjects were male. There was no statistical difference in age between the groups $\left(F_{2,52}=2.2, p=.14\right)$.

\section{Neuropsychological testing}

Average ADAS-Cog scores were: 19,6 (SD: 5,$9 ; n$ 15) in the AD group; 10,8 (SD: 4,5; $\mathrm{n} 20$ ) in the $\mathrm{MCl}$ group; and 5,8 (SD: 2,6; n 20) in the control group. These scores showed a significant difference between the groups in the expected direction $\left(F_{2,52}=84.8, p<.001\right)$. Results from the NART showed that the mean 


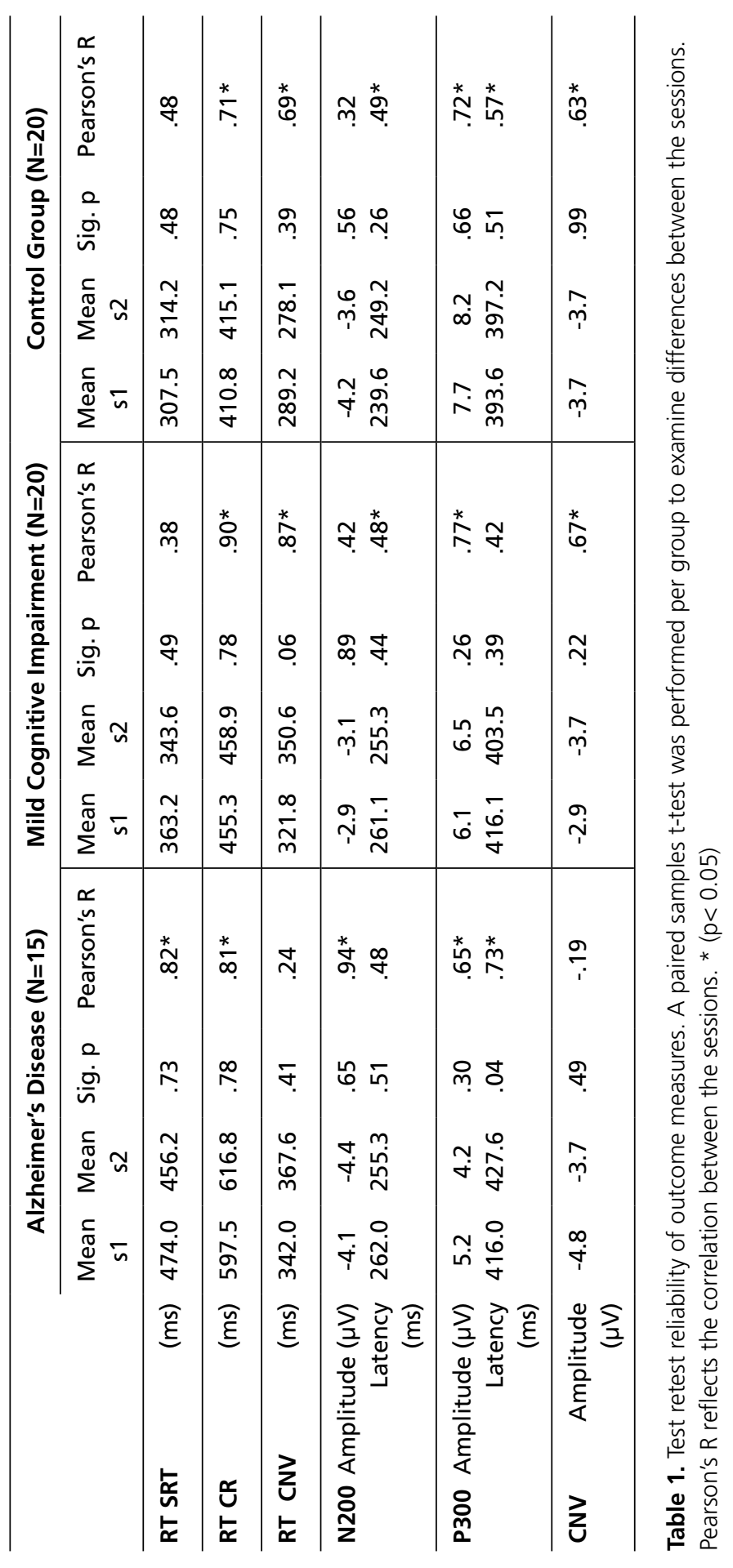


estimated pre-morbid intelligence was $96,9\left(F_{2,52}=2.3, p=1.03\right)$ and that it did not differ between the groups.

\section{Test-retest reliability}

The results from the TRR analysis are presented in table 1. This table shows the values measured on $\mathrm{Cz}$, which are comparable to the other midline electrodes.

The RS measured during the SRT, CRT and CNV task showed no significant difference between the test sessions. The correlation between the sessions for the RT measured during SRT was high for the AD group and moderate for the $\mathrm{MCl}$ and control group. The correlation between the sessions for the RT in the CRT was high to very high in all the patient groups.

The correlation between the sessions for the RT measured during CNV was high for the $\mathrm{MCl}$ and control group, but low for the AD group

The CNV amplitude showed no significant difference between the sessions, and the correlation between the sessions was high in the $\mathrm{MCl}$ and control group but low in the AD group. In the oddball paradigm, the P300 showed a significant difference between the sessions for latency in the $A D$ group. The correlation for P300 amplitude was high for all the groups. The P300 latency showed a moderate correlation between the sessions for the AD group and low correlation between the sessions for the $\mathrm{MCl}$ and control group. The N200 peak showed no differences between the sessions. The correlation was low except for the N200 amplitude in the AD group.

\section{Group differences response speed tasks}

The results of the RS tasks are presented in table 2. In the SRT, ANOVA analysis showed significantly longer reaction times in $A D$ compared to $\mathrm{MCl}$ and controls $\left(F_{2,52}=7.5, p=.002\right)$. Bonferonni post-hoc analysis showed that there was no significant difference between $\mathrm{MCl}$ and controls.

In the CRT, ANOVA analysis showed significantly longer reaction times in $A D$ compared to $\mathrm{MCl}$ and controls $\left(F_{2,52}=13.2, p<.000\right)$. Bonferonni post-hoc analysis showed that there was no significant difference between $\mathrm{MCl}$ and controls.

The reaction times measured in the CNV paradigm showed a significant difference between the groups $\left(F_{2,52}=4.17, p=.02\right)$. The RT was highest for the $A D$ group and lowest for the control group. Bonferonni post-hoc analysis showed that there was only a significant difference between the $A D$ and control group in this respect. 


\begin{tabular}{|c|c|c|c|}
\hline Component & Group & Amplitude $(\mu \mathrm{V})$ & Latency (ms) \\
\hline \multirow[t]{3}{*}{ CNV $^{n s}$} & $A D$ & $-3.8 \pm 2.7$ & N.A. \\
\hline & $\mathrm{MCl}$ & $-4.1 \pm 4.2$ & N.A. \\
\hline & Control & $-3.5 \pm 2.6$ & N.A. \\
\hline \multirow[t]{3}{*}{$P 300^{a, b}$} & $A D$ & $4.8 \pm 2.0$ & $433.6 \pm 51.5$ \\
\hline & $\mathrm{MCl}$ & $7.0 \pm 3.5$ & $422.9 \pm 34.7$ \\
\hline & Control & $8.4 \pm 3.1$ & $395.1 \pm 30.7$ \\
\hline \multirow[t]{3}{*}{ N200 ns } & $A D$ & $-3.9 \pm 5.3$ & $257.3 \pm 32.5$ \\
\hline & $\mathrm{MCl}$ & $-3.2 \pm 3.7$ & $253.1 \pm 29.3$ \\
\hline & Control & $-3.7 \pm 3.4$ & $247.1 \pm 27.8$ \\
\hline \multirow[t]{3}{*}{ Reaction time (CNV) } & $A D$ & $342.1 \pm 66.9 \quad$ (ms) & \\
\hline & $\mathrm{MCl}$ & $302.5 \pm 54.2 \quad$ (ms) & \\
\hline & Control & $287.4 \pm 59.2$ & \\
\hline \multirow[t]{3}{*}{ Reaction time (SRT) ${ }^{c}$} & $A D$ & $464.31 \pm 192.6(\mathrm{~ms})$ & \\
\hline & $\mathrm{MCl}$ & $355.79 \pm 81.2 \quad(\mathrm{~ms})$ & \\
\hline & Control & $305.47 \pm 45.0 \quad(\mathrm{~ms})$ & \\
\hline \multirow[t]{3}{*}{ Reaction time (CRT) } & $A D$ & $645.71 \pm 210.5(\mathrm{~ms})$ & \\
\hline & $\mathrm{MCl}$ & $459.95 \pm 87.9$ (ms) & \\
\hline & Control & $411.22 \pm 56.7 \quad(\mathrm{~ms})$ & \\
\hline
\end{tabular}

Table 2. Event related potential amplitude and latency and reaction time.

All measures from $\mathrm{Cz}$ electrode except P300 (Pz)

a Significant difference between the groups in amplitude in targets $(p<.05)$

b Significant difference between the groups in latency in targets $(p<.05)$

c Significant difference in RT between the groups $(p<.05)$

ns $N o$ significant between group differences

N.A. Not applicable 


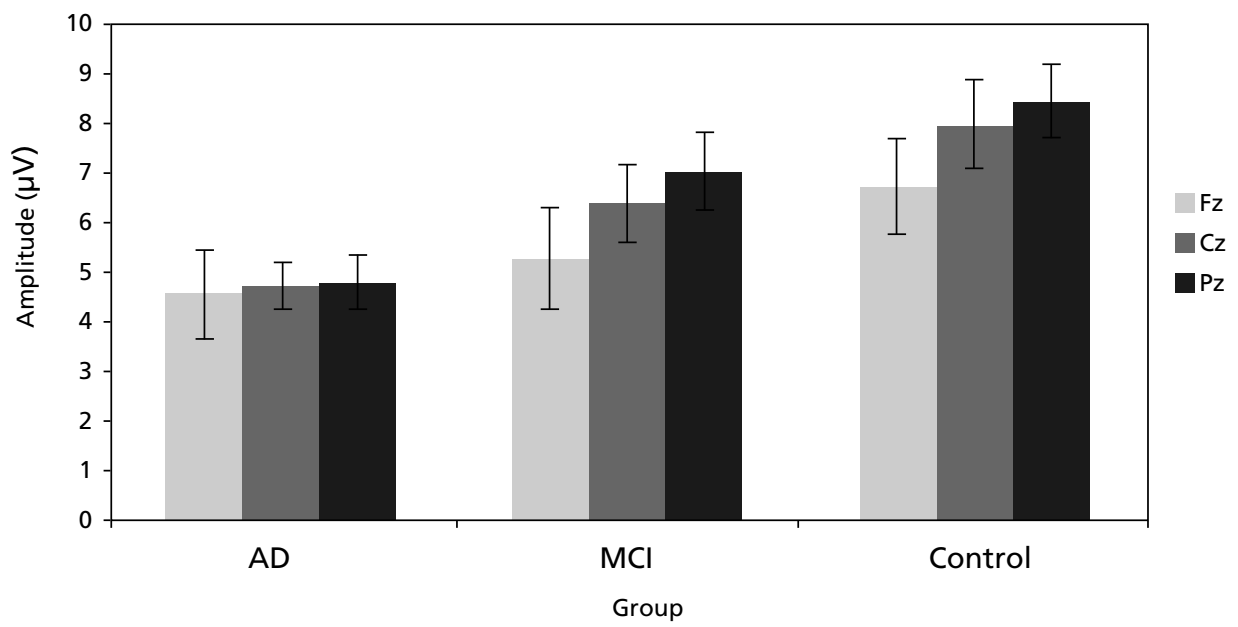

Figure 1. P300 amplitudes for $\mathrm{Fz}, \mathrm{Cz}$ and Pz. Differences are significant $(p<.05)$ for $\mathrm{Cz}$ and Pz between the $A D$ and control group.

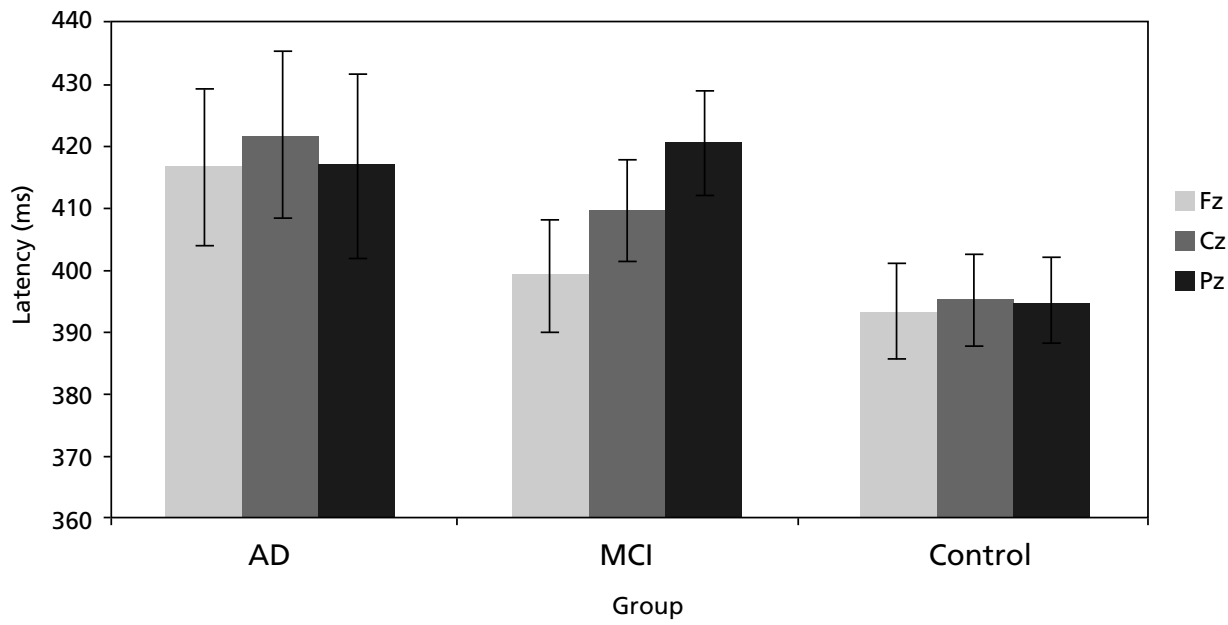

Figure 2. $P 300$ latency for $F z, C z$ and $P z$. Differences are significant $(p<.05)$ for $F z$ and $C z$ between the $A D$ and control group and for $\mathrm{Pz}$ between the $\mathrm{MCl}$ and control group.

\section{Group differences CNV paradigm}

The results of the ERP analysis for the CNV paradigm are presented in table 2. The analysis showed that the task provoked a CNV complex in all subjects. There were, however, no statistical differences between the $A D, M C l$ and control group for the amplitude of the CNV complex $\left(F_{2,52}=0.06, p=0.94\right)$, as can be seen in table 2 . The average number of 'clean' EEG trails that could be included 
in the analysis were; $A D: 24, \mathrm{MCl}: 26$, controls 29 . The number of trails was significantly lower for the $A D$ group compared to the $\mathrm{MCl}$ and control group $\left(F_{2,52}=3.7, p=.036\right)$.

\section{Group differences oddball paradigm}

The results of the ERP analysis for the oddball paradigm are presented in table 2. Figure 1 and figure 2 show the mean amplitudes and latencies of P300 over $\mathrm{Fz}, \mathrm{Cz}$ and Pz. The P300 showed a significant difference between the groups for amplitude at $C z\left(F_{2,52}=4.0, p=.025\right)$, and $P z\left(F_{2,52}=5.94, p=.005\right)$. Post-hoc Bonferonni showed that the amplitudes at $\mathrm{Cz}$ and $\mathrm{Pz}$ separated the $\mathrm{AD}$ group from the control group. The P300 latency was significantly longer for the AD and $\mathrm{MCl}$ group compared to the control group $\left(F_{2,52}=4.77, p=.013\right)$. Post-hoc Bonferonni showed that $\mathrm{Fz}$ and $\mathrm{Cz}$ separated the $\mathrm{AD}$ and control groups. The $\mathrm{Pz}$ electrode separated the $\mathrm{MCl}$ and control group. There was no significant difference in latency between the $A D$ and $\mathrm{MCl}$ group. The N200 component was analysed to ensure that P300 differences are not related to amplitude and latency differences in the N200 component. The N200 showed no significant differences between the groups on amplitude $\left(F_{2,52}=1.72, p=.189\right)$ and on latency $\left(F_{2,52}=1.54, p=.223\right)$. The average number of 'clean' trails that could be used for the analysis of the P300 and N200 components was; AD: 25, MCl: 27, controls: 28. There was no significant difference in the number of 'clean' trails between the groups $\left(F_{2,52}=2.1, p=.132\right)$.

\section{Speed of response execution}

An aspect that was not captured with the response speed task or ERP is the speed of response execution. As a post-hoc analysis we subtracted P300 latency from the reaction time in the CRT to capture this aspect. This analysis showed that this motor component takes significantly longer in the AD group compared to $\mathrm{MCl}$ and control groups $\left(\mathrm{F}_{2,52}=7.86, \mathrm{p}=.001\right)$.

\section{Correlation between reaction time and $C N V$ and $P 300$}

The correlation between RS and the CNV was analysed and showed no significant correlation between these measures, as can be seen in table 3 .

The correlation between the RS measured in the CRT and P300 amplitude showed only a significant negative correlation at Fz, Cz and Pz. The P300 latency showed a significant correlation at Fz only, as can be seen in table 3 . 
Functional and structural brain markers of Alzheimer's disease | Chapter 5

\begin{tabular}{lccc}
\hline & & Pearson's R & Sig. $\mathbf{p}$ \\
\hline CNV amplitude & $\mathrm{Fz}$ & .14 & .367 \\
& $\mathrm{Cz}$ & .24 & .113 \\
& $\mathrm{Pz}$ & -.19 & .212 \\
\hline P300 amplitude & $\mathrm{Fz}$ & -.38 & $.011^{*}$ \\
& $\mathrm{Cz}$ & -.39 & $.009^{*}$ \\
& $\mathrm{Pz}$ & -.42 & $.004^{*}$ \\
\hline P300 latency & $\mathrm{Fz}$ & .30 & $.047^{*}$ \\
& $\mathrm{Cz}$ & .24 & .235 \\
& $\mathrm{Pz}$ & .14 & .875 \\
\hline
\end{tabular}

Table 3. Correlations between the RT and ERP's.

The RT in the CNV is measured during the CNV paradigm.

The P300 is correlated to the RT in the CRT. * $(p<0.05)$

\section{Correlation with ADAS-cog}

The correlations between ADAS-cog and the outcome measures of the current study are presented in table 4. The RS measured in the SRT and CNV tasks show a moderate correlation with the ADAS-cog score. The RS measured during the CRT showed a high correlation with ADAS-cog.

The CNV amplitude showed a moderate negative but significant correlation at the $\mathrm{Cz}$ and Pz electrodes with ADAS-cog. The P300 amplitude showed a moderate but significant correlation with ADAS-cog on $\mathrm{Cz}$ and Pz. The P300 latency showed a significant correlation with ADAS-cog only at Pz.

\section{Discussion}

The main result of the current study is that RS as measured with SRT and CRT is slower in $\mathrm{AD}$ patients than in $\mathrm{MCl}$ and controls. Furthermore, group differences in RS are associated with differences in the P300 amplitude and latency.

The most prominent difference between the groups was increased RT in $A D$ in the CRT, a finding congruent with earlier studies (Gordon and Carson, 1990; Levinoff et al., 2005). There was no difference between $\mathrm{MCl}$ and controls on RT in any of the RS tasks, which is in contrast with Levinoff et al. (2005). The patient groups in Levinoff's study and the current one are comparable with regard to the age, MMSE and years of education. However, the RS in the MCI and control group in Levinoff's study were markedly slower than in the current study, which might be explained by more extensive practice of the participants 


\begin{tabular}{lccl}
\hline & & Pearson's R & Sig. $\mathbf{p}$ \\
\hline SRT & & .41 & $.005^{*}$ \\
\hline CRT & & .72 & $.000^{*}$ \\
\hline RT (CNV) & .37 & $.015^{*}$ \\
\hline CNV amplitude & Fz & -.27 & .067 \\
& $\mathrm{Cz}$ & -.33 & $.025^{*}$ \\
& $\mathrm{Pz}$ & -.37 & $.010^{*}$ \\
\hline P300 amplitude & $\mathrm{Fz}$ & -.13 & .351 \\
& $\mathrm{Cz}$ & -.29 & $.032^{*}$ \\
& $\mathrm{Pz}$ & -.44 & $.001^{*}$ \\
\hline P300 latency & $\mathrm{Fz}$ & .21 & .131 \\
& $\mathrm{Cz}$ & .22 & .110 \\
& $\mathrm{Pz}$ & .27 & $.047^{*}$ \\
\hline
\end{tabular}

Table 4. Correlation between the outcome measures and ADAS-cog score. The correlation coefficient is reflected by Pearson's R.

* $(p<0.05)$.

in the current study. The SRT and CRT further showed a satisfying TRR, a result comparable to an earlier study (Simpson, 1991). Therefore we can conclude that RS tasks can reliably be used in the case of an older, cognitively impaired, population.

The P300 amplitude was reduced, and the latency increased in AD, which is in agreement with previous findings (Golob and Starr, 2000; Goodin and Aminoff, 1992; Polich and Corey-Bloom, 2005; Polich et al., 1990). Although decreased P300 amplitude and increased P300 latency are consistent findings in $A D$, some studies did not find differences in amplitude, latency or both (Duffy et al., 1984; Polich and Pitzer, 1999; Verleger et al., 1992). The TRR of the findings was satisfactory and in the same order of magnitude as reported earlier (Polich and Herbst, 2000). More importantly, the changes in P300 amplitude and latency were associated with changes in RS as measured by CRT. The correlation between P300 amplitude and latency and RS were analysed for the CRT only, as both the CRT and the oddball task require stimulus characterisation (ie left/right the CRT and target/non-target in the oddball paradigm). The other RS tasks; SRT and CNV did require such stimulus classifications.

High amplitudes and short latencies of the P300 were associated with faster responses. This relation has been shown in healthy subjects (Dimoska et al., 2006; Holm et al., 2006) and an MCl population, but not in AD (Williams et al., 1991). 
The P300 amplitude is sensitive to the amount of attention that is allocated for the detection of a stimulus. In undemanding tasks, such as the oddball paradigm, processing resources are focussed on detecting the deviant stimulus, resulting in higher P300 amplitude. When task demands increase, for example by more types of stimuli to attend to, the processing resources are allocated to more different types of stimuli, resulting in a lower P300 amplitude and a longer latency (Polich, 2007). The general state of arousal also affects the P300 amplitude as passive processing of stimuli generally produce smaller P300 amplitudes than active stimulus processing (Polich, 2007). The decreased P300 amplitude in AD during the oddball task, suggests that the amount of attentional resources that are focussed on the detection of the deviant stimulus is lower. The increased P300 latency further suggests that the processes involved in evaluating and categorizing the stimulus is less efficient in AD (Bashore et al., 1989). The current results provide support for the hypothesis that reduced access to attentional resources and impaired stimulus classification are relevant in explaining longer reaction times in AD.

The CNV amplitude showed no difference between the groups, which is in contrast with earlier studies (Golob and Starr, 2000; Zappoli et al., 1987). Furthermore, was there no significant correlation between the CNV amplitude and RS measured during CNV, while this would have been expected based on earlier studies (Brunia and Vingerhoets, 1980; Brunia and Vingerhoets, 1981; Haagh and Brunia, 1985). Since the length of the inter-stimulus interval affects the CNV amplitude (Van der Lubbe et al., 2004), the lack of a difference between the groups in the current study might be related to the short inter-stimulus interval (ISI) (i.e. two seconds) used. In an earlier pilot study we found that an ISI of four seconds was too long for the AD patients (data not published). Furthermore, the TRR of the CNV in the AD group was low. Despite extensive practice during each visit prior to the measurement, some patients especially in the AD group were not able to perform the CNV paradigm properly, resulting in a significantly smaller number of viable trials and less reliability. Another explanation might be that the low TRR is related to the relatively long time lag compared to other ERP (e.a. two seconds) between the warning stimulus and the CNV peak. This long time interval might induce more variance and noise, especially in the AD group. The methodological issues together with the low TRR make it impossible to conclude whether anticipatory behaviour as defined by CNV, has an effect on the slowed RS in AD.

The present study further showed that there was a significant difference between the groups in the speed of response execution. These results suggest that impaired response execution contributes to the slowed RS in AD. The finding that the motor component of response execution is slowed in $A D$ is in agreement with previous work (Bellgrove et al., 1997; Camarda et al., 2007). 
The correlation between ADAS-cog and the other measures in this study was highest for the CRT. Although the ADAS-cog does not specifically measure speed, attentional or executive processes, it is plausible that the performance of these processes have an effect on the overall performance on ADAS-cog. This probably explains the high correlation between CRT and ADAS-cog performance. The CNV amplitude showed no significant correlation with ADAS-cog, which is not surprising since it did not separate the patient groups. The P300 amplitude and latency showed a moderate correlation with ADAS-cog, which was comparable with a previous report of the correlation between P300 and MMSE (Bennys et al., 2007).

A weakness of the current study was its cross-sectional design that did not allow us to evaluate the sensitivity of the measures for changes in longitudinal studies. Another weakness is that all subjects were recruited in a University tertiary reference centre. Therefore the results may not be applicable to the general $\mathrm{AD}$ and $\mathrm{MCl}$ population. A strong aspect of the study was that patients were accurately diagnosed in accordance with standardized research criteria. Another strong aspect was that none of the subjects used psychoactive or cholinesterase inhibiting drugs that could have biased the results. Furthermore, the TRR design provided insight in the reproducibility of the endpoints.

The current study showed that especially the stimulus evaluation and the response execution component are impaired in $A D$. The results furthermore show that RS measures are reproducible and sensitive in detecting differences in $A D$ compared to $\mathrm{MCl}$ and controls. Altogether, the current study suggests that RS measures on a behavioural and psycho-physiological level deserve more attention as a possible marker for the diagnosis and to asses changes in AD.

\section{Acknowledgements}

This work was financially supported by research agreement CSMD Ref No: $\underline{002509}$ from GlaxoSmithKline, Cambridge, UK. 



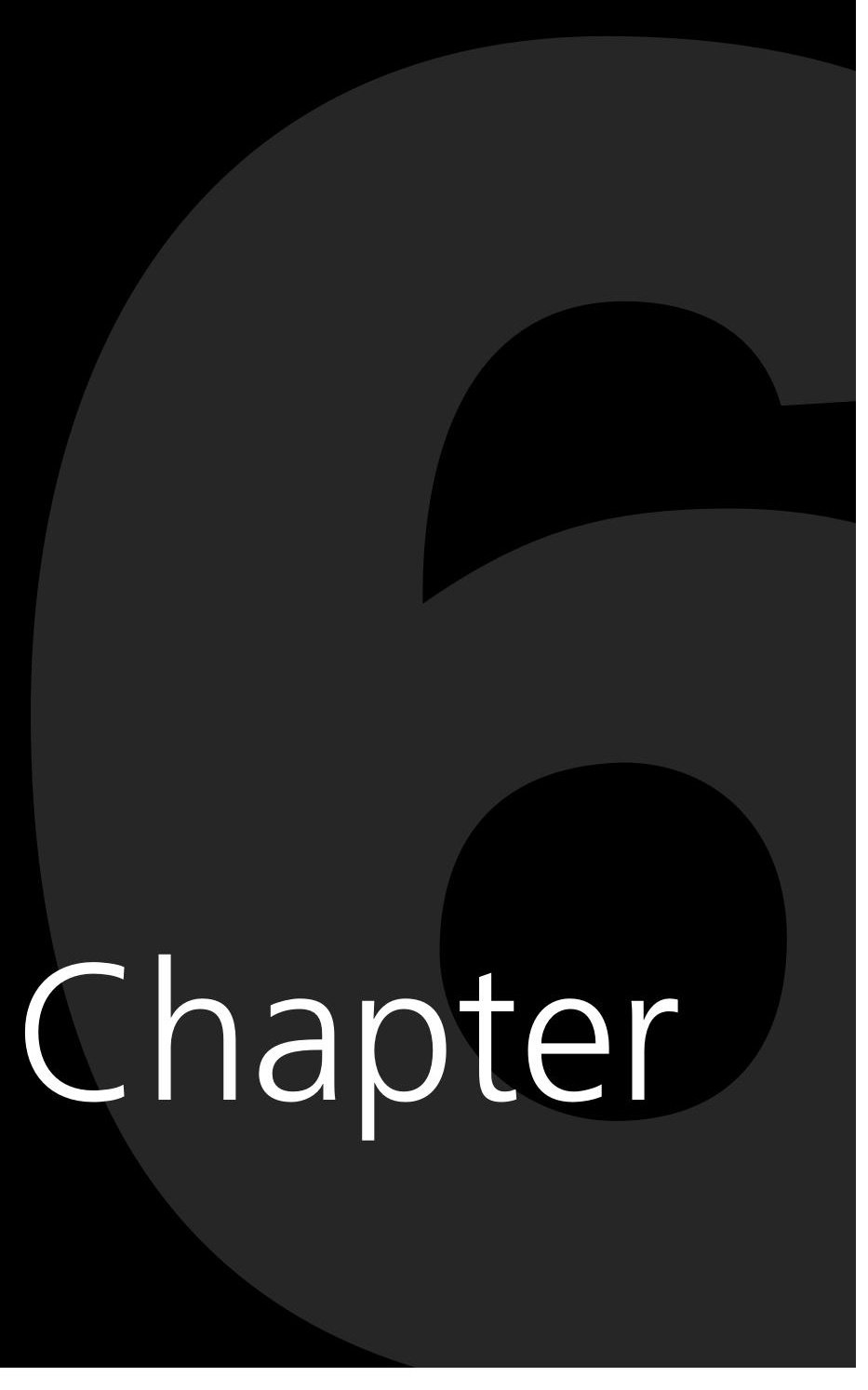





\title{
Comparison of EEG paradigms in the diagnosis of Alzheimer's disease and $\mathrm{MCl}$
}

\author{
Submitted for publication
}

J.A. van Deursen, E.F.P.M. Vuurman, F.R.J. Verhey, W.J. Riedel

\begin{abstract}
Aims: Alzheimer's disease (AD) is the most common form of dementia. In recent years many studies have shown that $A D$ is characterized by changes that can be detected with the electroencephalography (EEG). At present it is unclear to what extent EEG measures can be used to identify $A D$ and to monitor its progression. The aim of the present study was to determine which of the EEG paradigms are the most sensitive, specific and reliable when it comes to discriminating between subjects with $\mathrm{AD}$ or $\mathrm{MCl}$ and healthy controls.

Methods: Fifteen AD patients, twenty $\mathrm{MCl}$ patients and twenty healthy control subjects, all naïve for the use of psychoactive medication were included in this study. To evaluate test-retest reliability all participants were tested in two identical sessions. The EEG paradigms asessed were: gamma-band power during resting state, music listening, story listening and visual stimulation. Further measures were taken of delta, theta, alpha and beta power during resting state; $40-\mathrm{Hz}$ steady-state responses; the P300; and contingent negative variation amplitude. In addition to the EEG, a simple and a choice reaction time task as well as the ADAS-cog were measured.

Results: The results show that the response speed tasks and the ADAS-cog were
\end{abstract}


most accurate and reliable in discriminating subjects with $A D$ from $M C l$ and healthy controls. Of the EEG paradigms studied, measures of the P300, of the gamma-band power during music listening, and of the delta power during resting state were most accurate and reliable in discriminating between patient groups. Conclusion: Based on the present findings we can conclude that EEG has no additional value as a diagnostic instrument. However, in the follow-up of disease progression and treatment intervention several EEG paradigms are promising candidate biomarkers.

\section{Introduction}

Alzheimer's disease (AD) is the most common form of dementia. In recent years many efforts have been made to develop biomarkers that can provide more insight into its pathology, enhance diagnostic accuracy and be used to evaluate treatment interventions. The electroencephalogram (EEG) is one of the measures that have been studied extensively.

A major advantage of EEG over other biomarkers is that it directly measures neurological activity, whereas other functional measures such as single photon emission tomography (SPECT), positron emission tomography (PET) and functional MRI mainly reflect perfusion and the glucose or oxygen metabolism of the brain. Several neurophysiological measures have been tested as biomarkers, especially continuous EEG and event-related potentials (ERPs).

A large number of studies have indicated that an EEG of a subject with $A D$ is characterized by changes in the frequency spectrum as well as by changes of the event-related potentials (ERPs). Most studies that assessed frequency changes in $A D$ have found that the power in the lower frequency spectra increases while the power in the higher spectra decreases (Huang et al., 2000; Jelic et al., 1996; Jeong, 2004). Studies that focused on the highest EEG frequency spectrum (i.e. gamma band, 30-100 Hz), showed a decline in synchronization (Stam et al., 2002), but also increased gamma-band activity (van Deursen et al., 2008).

The ERP that has been studied most frequently in subjects with $A D$ is the P300. This ERP is usually elicited in oddball paradigms in response to deviant visual or auditory stimuli. The amplitude of the P300 is considered to be the manifestation of brain activity reflecting attention to incoming stimuli when representations are updated. The P300 latency is considered to be a measure of stimulus classification speed (Polich, 2007). The P300 in AD is characterized by decreased amplitude and by an increased latency (Polich et al., 1990). 
Besides its use as a diagnostic instrument, EEG may also be of interest as a potential marker to monitor the effects of treatment interventions. To date, most therapeutic agents in $A D$ focus on the cholinergic nervous system. The sensitivity of changes in EEG frequency and ERPs to cholinergic treatment interventions has been established in several studies (Adler et al., 2004; Babiloni et al., 2006; Werber et al., 2001; Werber et al., 2003). Currently the Alzheimer's disease assessment scale (ADAS-cog) (Rosen et al., 1984; Verhey et al., 2004) is considered to be the gold standard for the evaluation of therapeutic interventions. Recently the ADAS-cog has received some criticism because of the subjective nature of several subscales, its relative insensitivity and its failure to detect decline in placebo treated patients in 6-month follow-up studies (Wesnes, 2008). Furthermore, a recent study showed that response speed is more sensitive to therapeutic changes than ADAS-cog (Caramelli et al., 2004). It is therefore interesting to study the additional value of EEG and response speed parameters as biomarkers to monitor treatment effects.

Several of the EEG paradigms have proven to be useful in identifying differences between patients with $A D, M C l$ and healthy control subjects. Of several options, it remains unclear, which of the EEG paradigms are the most sensitive, specific and reliable. Demographic variables such as age, disease severity and use of psychoactive substances often varied between studies. Since age, disease severity and psychoactive drug use have a profound effect on the EEG frequency and ERP components, the variance between studies makes it difficult to compare the outcomes head to head (Babiloni et al., 2006; Bennys et al., 2007; Kowalski et al., 2001; Werber et al., 2003). As a result, there is not enough information about which of these electrophysiological paradigms are the most reliable, sensitive and specific when it comes to discriminating AD patients from healthy controls, $\mathrm{MCl}$ patients and subjects with other forms of dementia. This type of information is necessary to determine which of the EEG paradigms are most viable as an electrophysiological biomarker.

The present study aims to determine which EEG paradigms are the most sensitive, specific and reliable. The study examined measures of: four gammaband power paradigms; the delta, theta, alpha and beta power during resting state; a 40-Hz steady-state response (SSR) paradigm; an oddball paradigm; and a contingent negative variation (CNV) paradigm. Besides these EEG paradigms, the sensitivity, specificity and reliability of two response speed tasks and the ADAScog were also investigated. To ensure that effects of psychoactive medication did not bias the results of the present study, only patients who were naïve for current or former use of psychoactive medication were included. 


\section{Material and Methods}

\section{Subjects}

The study involved three different groups of subjects:

1. Fifteen patients with a diagnosis of probable AD according to the NINCDSADRDA criteria were included, who were naïve for the use of psychoactive medication (McKhann et al., 1984).

2. Twenty subjects who fulfilled the Petersen criteria for $\mathrm{MCl}$ and who were naïve for the use of psychoactive medication were included (Petersen et al., 2001). Classification of $A D$ or $\mathrm{MCl}$ was made at a weekly consensus meeting of different specialists. The diagnostic assessment included the following: a detailed history-taking of the subjects; a psychiatric, neurological and physical examination; the Mini Mental State Examination (MMSE) (Folstein et al., 1975c); CAMDEX part-B (CAMCOG) (Derix et al., 1991); an assessment using clinical rating scales (i.e. GDS (Reisberg et al., 1982), the Hamilton Depression scale (Hamilton, 1960) and the Blessed Dementia Rating scale (Blessed et al., 1968); appropriate laboratory tests (i.e. haematology, glucose, biochemical analysis, vitamin B12 and thyroid stimulating hormone); a neuropsychological assessment including tests covering the domains of memory, attention, executive functioning, language, praxis and intelligence (Verhey et al., 1993); and CT or MRI imaging as described elsewhere (Verhey et al., 1993).

3. Twenty healthy control subjects were recruited from the Maastricht Aging Study (MAAS), a longitudinal study of the determinants of healthy cognitive aging (van Boxtel et al., 1998). The control subjects were not using any psychoactive medications. Their medical history was screened with the aid of a medical health questionnaire. Main exclusion criteria for all participants were: a history of stroke, head trauma, or any other neurological or psychiatric disorder. Additional exclusion criteria were: severe cardiovascular disease, a Hachinski Ischemic Scale (HIS) (Hachinski et al., 1974; Rosen et al., 1980) higher than three and/or a history of substance abuse and/or other serious system diseases (e.g. malignancy, uncontrolled hypertension, neuropathy or seizure disorders). All $A D$ and $\mathrm{MCl}$ patients were recruited at the Memory Clinic of the Maastricht University Hospital and were judged competent to give consent by their treating physicians. All participants gave written informed consent prior to the study; in the case of AD patients a family member also signed the consent form. This study was carried out in accordance with the declaration of Helsinki. The local Medical Ethics Committee of the Maastricht University Hospital approved the study. 


\section{Behavioural measures}

The ADAS-cog (Rosen et al., 1984) and MMSE (Folstein et al., 1975c) were used to evaluate the cognitive performance of all subjects. For the current study a European validated version of the ADAS-cog was used (Verhey et al., 2004). This version of the ADAS-cog assesses several cognitive domains and contains a 12-item scale with a score range of 0-75 (i.e. higher scores indicate greater cognitive impairment).

The National Adult Reading Test (NART) is a reading test that yields an estimated score of a subject's pre-morbid verbal IQ (Schmand et al., 1998). The NART was included to control for potential confounding differences in pre-morbid IQ between the groups in the current study.

\section{Simple and Choice reaction time tasks}

The simple reaction time task (SRT) was presented in four runs of 12 trials each. In each trial of the SRT the presentation of a white square $(4 \times 4 \mathrm{~cm})$ on a black background was followed by a red square $(4 \times 4 \mathrm{~cm})$. Subjects were instructed to push the response button as fast as possible when the white square turned red. After the response the red square turned white again until the next trial. The interval between the trials varied between two and six seconds. Between the runs there was a break of one minute.

The choice reaction time task (CRT) was presented in four runs of 12 trials. In each trial two white squares $(4 \times 4 \mathrm{~cm})$, one on the left side and one on the right side of the screen, were presented on a black background. When one of the two squares turned red the subject had to push the congruent response button, left or right. After the response the red square turned white again. The interval between the trials varied between two and six seconds. The visual stimuli were presented on a 16-inch computer screen. The responses were given on a PST Serial Response Box $^{\circledR}$ (for specifications see www.pstnet.com).

\section{EEG acquisition}

A commercially available Nuamps ${ }^{\circledR}$ EEG acquisition system was used to record the EEGs. Electrodes $(\mathrm{Ag} / \mathrm{AgCl})$ were positioned following the 10-20 system on a 32-channel electrode cap, using 19 Medcat $^{\circledR}$ electrodes. A low pass filter of $100 \mathrm{~Hz}$ and a high pass filter of $0,1 \mathrm{~Hz}$ were applied. The sample frequency was $512 \mathrm{~Hz}$ and analogue-digital conversion was 20 bit. Electrode impedance was kept below $5 \mathrm{k} \Omega$. A reference electrode was placed on the right ear lobe. To control for possible vertical eye movements, an electro-oculogram (EOG) electrode was placed one centimetre under the midline of the right eye. A ground electrode was placed on the forehead, at Fpz. EEGs were recorded in a magneti- 
cally shielded and sound attenuated room. The visual stimuli were presented on a flat, 16-inch LCD screen located outside the shielded room at eye height. Subjects were able to see the screen through magnetically shielded glass. Headphones were used to present the auditory stimuli. Neuroscan ${ }^{\circledR} 4.3$ software was used for EEG recording and analysis.

\section{EEG paradigms}

\section{EEG gamma-band paradigms}

Gamma-band activity was measured under the following four conditions: during resting state, music listening, story listening and visual stimulation. During the resting state the delta, theta, alpha and beta bands were also recorded.

During the resting state measurement subjects were instructed to keep their eyes open and focus on a white crosshair located on a black screen for 90 seconds.

During the music listening task, subjects were instructed to listen for 90 seconds to broad-spectrum acoustical music. While listening to the stimuli, subjects focused on a white crosshair on a black screen and kept their eyes open. The music was a segment from Enya's "From where I am".

During the story listening task subjects listened for three minutes to a fragment of a Dutch spoken documentary (Fly Away 2, fragment Australia). While listening to the stimuli subjects had to focus on a white crosshair on a black screen with their eyes open. The fragment was spoken monotonously and pauses were not longer than five seconds. Subjects were instructed to listen carefully to the spoken text.

In the visual task a series of moving abstract objects were presented for 180 seconds. The objects were coloured and moved at variable speed. This was a fragment of a recorded Windows ${ }^{\circledR}$ screensaver. Subjects were instructed to monitor the movements carefully.

\section{Event-related potentials}

\section{Oddball paradigm}

A simple auditory two-tone discrimination or 'oddball' paradigm was used to elicit ERP responses. Thirty-two targets consisting of high tones $(2000 \mathrm{~Hz}, 80 \mathrm{~dB}$, $100 \mathrm{~ms}$ ) were pseudo-randomly distributed within a stream of non-target low tones $(1000 \mathrm{~Hz}, 80 \mathrm{~dB}, 100 \mathrm{~ms})$. A fixed inter-stimulus interval of two seconds was used. Target and non-target stimuli appeared with a probability of $15 \%$ and $85 \%$ respectively. Subjects were requested to press a handheld response 
button when they detected a target stimulus. They were instructed to respond accurately without any emphasis on a speedy response in order to limit the possible contamination of the P300 with motor potentials. The task was practised for three minutes, prior to each recording session to prevent learning effects.

\section{CNV paradigm}

The visual CNV paradigm consisted of a warning stimulus in the form of a big red dot $(\varnothing 12 \mathrm{~cm})$, followed by the imperative stimulus in the form of a big green dot $(\varnothing 12 \mathrm{~cm})$. A total of 36 trials were administered, the inter-stimulus interval (ISI) was two seconds and the inter-trial interval (ITI) varied between five and ten seconds. The subjects were instructed to respond as fast as possible when the imperative stimulus appeared. The task was practised for three minutes prior to each recording session to prevent learning effects.

\section{0-Hz steady-state response}

The auditory $40-\mathrm{Hz}$ SSR task consisted of "click trains" of bursts of pure tones at a $40-\mathrm{Hz}$ frequency of 450-millisecond duration. In total, 80 click trains were presented binaurally. Subjects were instructed to focus on a white crosshair in the centre of a black screen during $40-\mathrm{Hz}$ stimulation.

\section{EEG analysis}

EEG analysis was performed off-line. For the frequency analysis during resting state, music listening, story listening and the visual task, the continuous EEGs were cut in epochs of one second. Epochs containing eye movement, electromyographic activity and head motion artefacts were excluded from analysis. The uncontaminated epochs were transformed from the temporal domain to the frequency domain using fast-Fourier transformation (1 Hz resolution, 512 point block-size, Hanning window 1-100 Hz). The delta band was defined as the frequency between $1-4 \mathrm{~Hz}$; the theta band was defined as the frequency between 4-8 Hz; the alpha band as the frequency between $8-13 \mathrm{~Hz}$; and the beta band as the frequency between $13-30 \mathrm{~Hz}$. These slower frequency bands were only calculated for the resting state condition. The gamma band was defined as the frequency between $30-100 \mathrm{~Hz}$ and was calculated for resting state, music listening, story listening and visual stimulation. A notch filter of $50 \mathrm{~Hz}$ was applied to omit contamination of the electrical light network in the room. The frequency epochs were averaged and absolute gamma-band power was calculated for every task, subject and electrode.

In the case of $40-\mathrm{Hz}$ SSR, epochs of the stimulation condition were made, each with a length of $450 \mathrm{~ms}$. Epochs containing eye movements, electromyo- 
graphic activity (EMG), or head motion artefacts were omitted from analysis. Pre-processing procedures included: band-pass filtering (35-45 Hz, $48 \mathrm{~dB} / \mathrm{oct}$ ) and baseline correction. The uncontaminated epochs were transformed from the temporal domain to the frequency domain using Fast-Fourier transformation ( $1 \mathrm{~Hz}$ resolution, 512 point block-size, Hanning window 35-45 Hz). The mean power at $40-\mathrm{Hz}$ was calculated for each subject and each session.

The analysis of the oddball paradigm included: high-pass filtering $(>1 \mathrm{~Hz}$ $12 \mathrm{~dB} /$ oct); ocular artefact reduction; low-pass filtering ( $<30 \mathrm{~Hz}, 12 \mathrm{~dB} / \mathrm{oct}$ ); baseline correction; and averaging. Peak latencies were determined relative to the stimulus onset. Amplitudes of the P300 were defined relative to a baseline period, i.e. $100 \mathrm{~ms}$ prior to stimulus onset. An automated peak-picking procedure was used to determine peak amplitudes and latencies. The P300 was defined as the maximum point between $300-600$ ms post stimulus. Mean P300 amplitudes and latencies were calculated for each subject and each session.

In the case of the CNV paradigm the interval of one second prior to the imperative stimulus was analysed. Pre-processing procedures included: ocular artefact reduction; low-pass filtering $(1-30 \mathrm{~Hz}, 12 \mathrm{~dB} / \mathrm{oct})$; baseline correction; and averaging of the data. The peak amplitude of the epochs was calculated for each subject. The reaction times were calculated relative to onset of the imperative stimulus. Mean reaction times over the 36 trials were calculated; trials with reaction times faster than $60 \mathrm{~ms}$ and slower than $600 \mathrm{~ms}$ were omitted from analysis.

\section{Experimental procedure}

All subjects took part in two identical recording sessions, spaced apart by one week. Prior to EEG acquisition, the cognitive subscale of the Alzheimer's Disease Assessment Scale (ADAS-cog) (Rosen et al., 1984; Verhey et al., 2004) was administered. The Dutch version of the National Adult Reading Test (NART) (Schmand et al., 1998) was administered only in the first session to estimate pre-morbid intelligence. A simple reaction time task (SRT) and a choice reaction time task (CRT) were performed to examine reaction times. The neurophysiological outcome measures in the current study were: gamma-band activity measured during music listening, story listening and movie watching; delta-band power, theta-band power, alpha-band power, beta-band power and gammaband power measured during resting state; evoked potentials measured with auditory $40-\mathrm{Hz}$ steady-state stimulation; contingent negative variation (CNV); and P300 elicited by an auditory oddball paradigm. To evaluate test-retest reliability (TRR) of the acquired data, all subjects were tested on two occasions, separated by one week. 


\section{Statistical analysis}

Statistical analysis was done with SPPS for Windows (version 11.5). To examine test-retest reliability of the variables, a paired T-test was used to evaluate potential differences between the two sessions. Furthermore, Pearson's correlation coefficient between the sessions was calculated for each endpoint. These analyses were done for each patient group separately.

The ROC method in SPSS 11.5 was used to asses the capacity of the variables to discriminate between $A D, M C l$ and control subjects. $R O C$ curves were created presenting sensitivity on the vertical axis and 1-specificity on the horizontal axis. The variables were considered best when the curves were drawn in the top-left area of the diagram. Variables that were located below the reference line (bottom right) were considered to perform poorly. The area under the curve $(A \cup C)$ was calculated as a quantitative measure of the discriminative properties of each variable. The ROC method involves calculating the sensitivity and specificity of the variables when it comes to discriminating between $A D$ and healthy controls and between $A D$ and $\mathrm{MCl}$. Sensitivity and specificity was considered to be low between 0-0.6, moderate between 0.6-0.8 and high between 0.8-1.0.

Since the present study compares a substantial number of EEG paradigms, the described statistics were applied to a limited number of electrodes. We chose to analyse electrodes that showed group differences in previous studies. In the case of the gamma-band tasks, a previous study showed differences mainly at Pz, P3, P4, $O 1$ and O2 (van Deursen et al., 2008). In the case of the delta, theta, alpha and beta power the Fz, F3 and F4 electrodes were analysed. In the case of the $40-\mathrm{Hz}$ SSR a previous study showed differences at T5, T6 and $\mathrm{O} 2$ (van Deursen et al., 2009). In the case of the oddball and CNV paradigms, results from the $\mathrm{Fz}, \mathrm{Cz}$ and $\mathrm{Pz}$ electrodes are presented.

\section{Results}

\section{Subjects' demographics}

Fifteen AD patients had a mean age of 75.2 (SD 6.9) and a mean MMSE of 20.8 (SD 2.7, range 17-26). Eleven of the $A D$ patients were male.

Twenty $\mathrm{MCl}$ patients were included, with a mean age of 70.6 (SD 7.2) and a mean MMSE of 26.3 (SD 1.6, range 23-29). Twelve of the MCI patients were male.

Twenty healthy control subjects were included, with a mean age of 69.5 (SD 6.1) and a mean MMSE of 29.3 (SD 0.8, range 28-30). Twelve of the healthy control subjects were male. There was no statistical difference in age between 
the groups $\left(F_{2,52}=2.2, p=.14\right)$. The NART showed that the mean estimated pre-morbid intelligence was 96,9 and this did not differ between the groups $\left(F_{2,52}=2.3, p=1.03\right)$.

\section{Test-retest reliability}

The results from the test-retest reliability analysis are presented in table 1 . The analysis showed that the ADAS-cog has a high TRR, especially in the AD group. In the reaction time task the TRR of the CRT is higher than that of the SRT. The SRT shows a high TRR in the AD group only. The frequency analysis of the theta, alpha and beta waves measured during resting state showed a very high TRR. The delta wave showed lower test-retest reliability in the AD group, but a high TRR in the $\mathrm{MCl}$ and control group. The frequency analyses in the gamma band showed a high TRR during resting state, music listening and story listening. The TRR of GBP during the visual task was markedly lower than that of the other three gamma band paradigms. In the CNV paradigm the TRR was satisfactory in the $\mathrm{MCl}$ and control group, but it was low in the AD group. The $40-\mathrm{Hz}$ SSR showed a significant difference between the sessions for $\mathrm{T} 5$ in the $\mathrm{MCl}$ group. Furthermore, the correlation between the sessions was high in the $A D$ and $\mathrm{MCl}$ group and moderate in the control group. In the oddball paradigm, the P300 showed a significant difference between the sessions for latency in the AD group. The correlation for P300 amplitude was high for all the groups. The P300 latency showed a moderate correlation between the sessions.

\section{Comparison between the $A D$ and control group}

Table 2 presents the sensitivity, specificity and AUC of the comparison between $A D$ and healthy controls. The ROC curves presented in figure 1 illustrate the sensitivity and specificity of the discrimination between the AD and the control group. The cognitive measures ADAS-cog, MMSE and CRT show a very high sensitivity and specificity in discriminating $A D$ patients from healthy controls.

The delta band during resting state showed a moderate sensitivity and specificity in discriminating AD patients from healthy controls. The theta band during resting state showed a moderate sensitivity and specificity in discriminating $A D$ patients from healthy controls. The alpha band showed a moderate sensitivity and a low specificity in discriminating AD patients from healthy controls. The beta band showed a moderate sensitivity and a low specificity in discriminating $A D$ patients from healthy controls.

The gamma-band power during resting state showed a high sensitivity for 


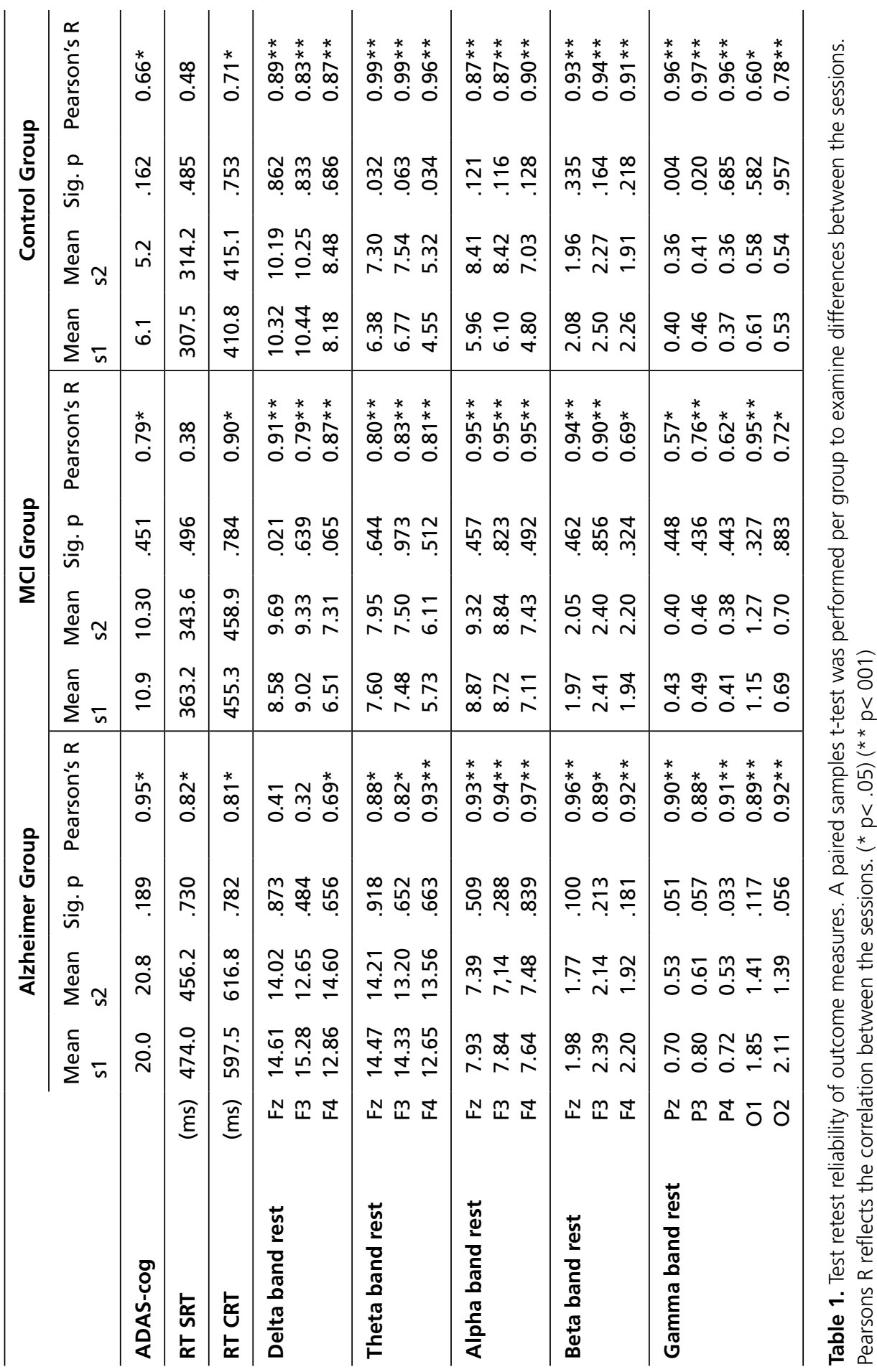


Functional and structural brain markers of Alzheimer's disease | Chapter 6

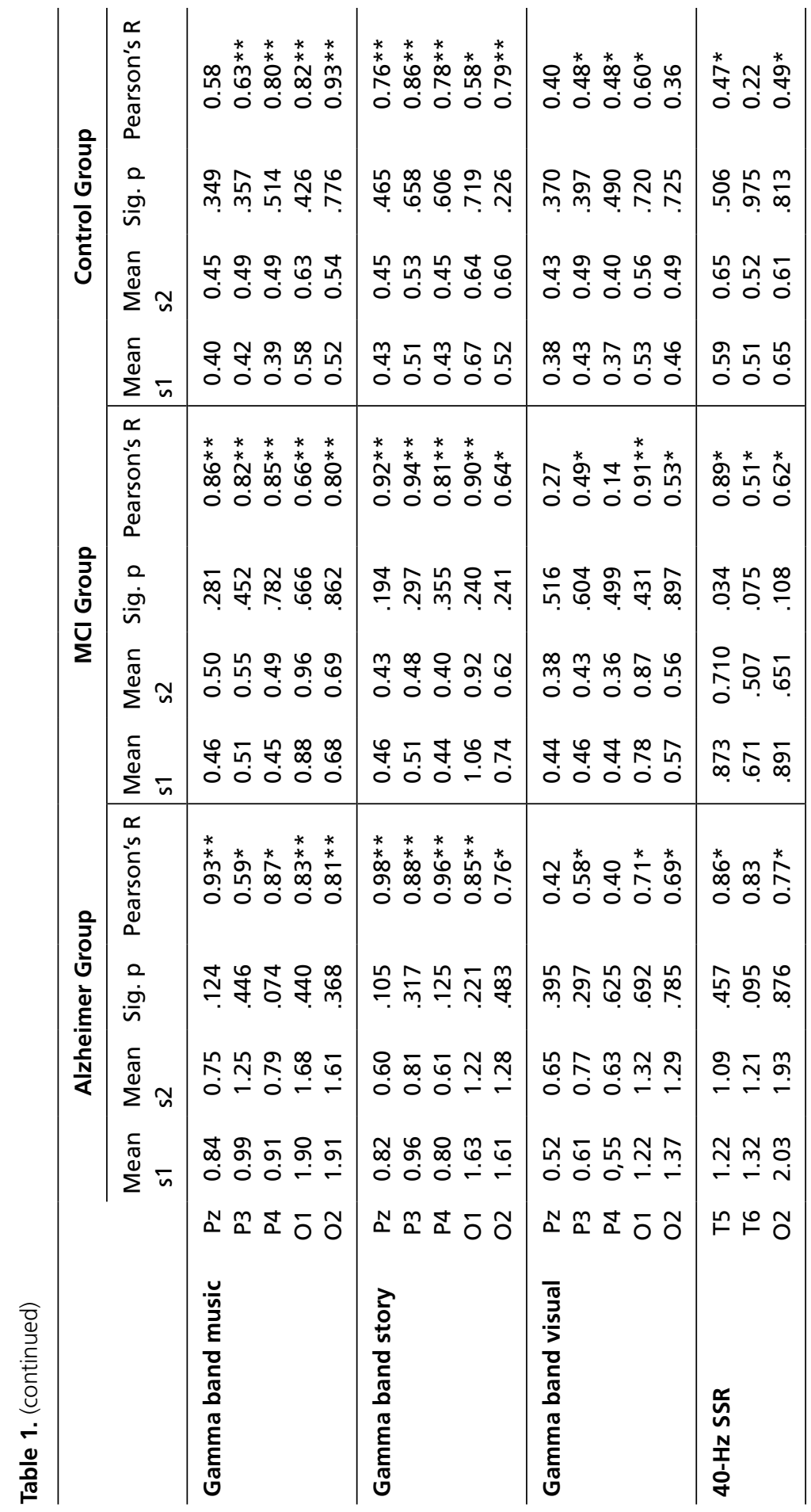




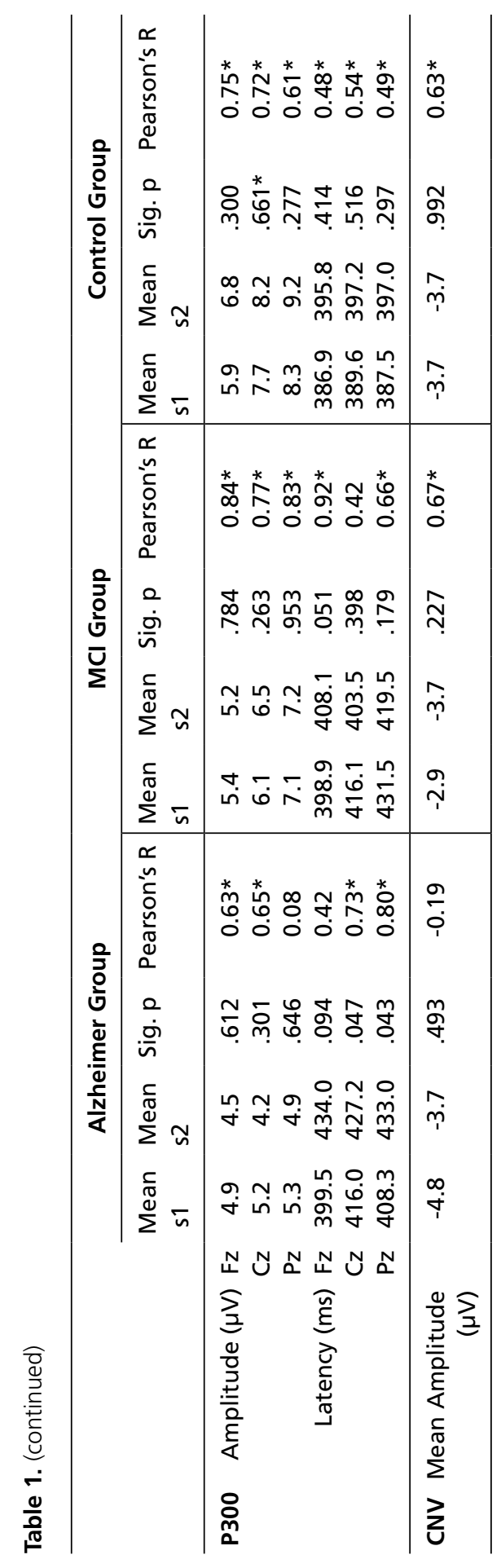



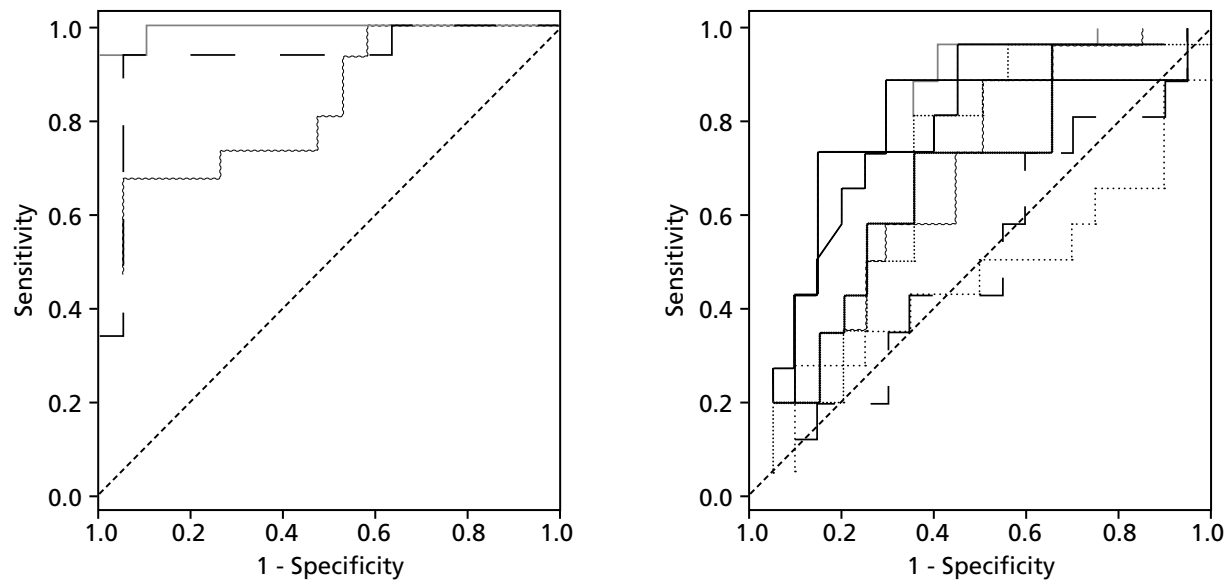

Source of the curve

$$
\begin{aligned}
& - \text { ADAS_M } \\
& - \text { SRT_M } \\
& -- \text { CRT_M } \\
& -\cdots \text { Reference Line }
\end{aligned}
$$

$$
\begin{aligned}
& \text { Source of the curve } \\
& \text { - Rest_delta_F3 Music_gamma_P3 } \\
& \text { Rest_theta_F3 } \\
& \text { - - Rest_alpha_F3 } \\
& \text { ….... Rest_beta_F3 } \\
& \text { Story_gamma_P3 }
\end{aligned}
$$
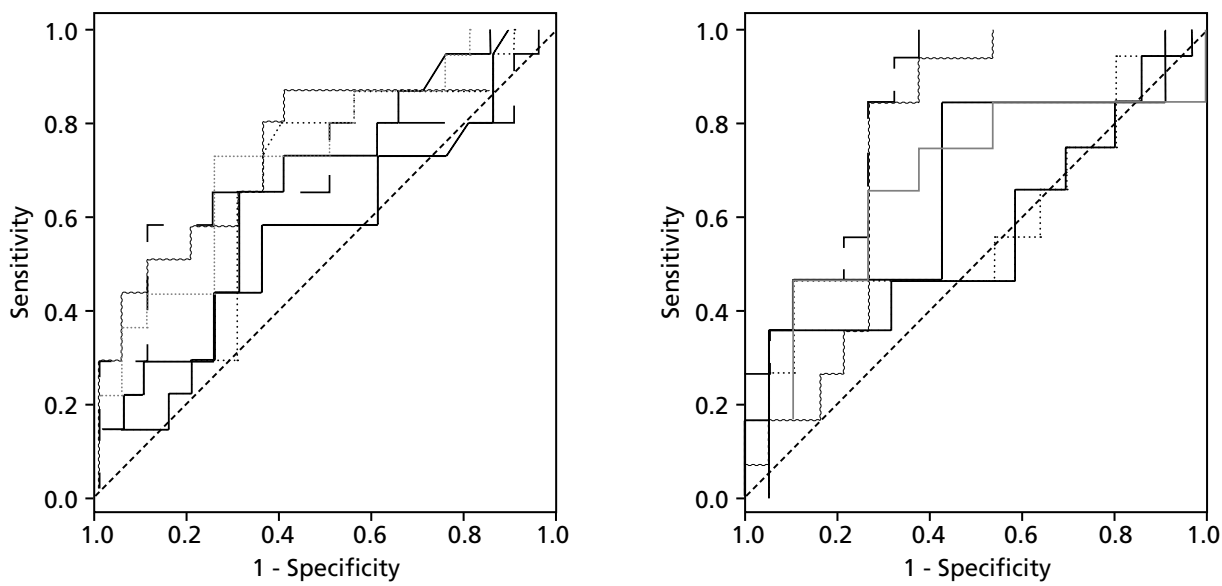

Source of the curve

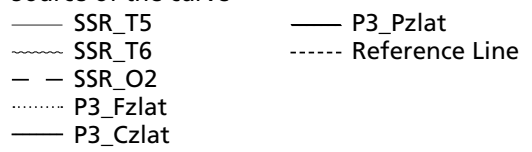

Source of the curve

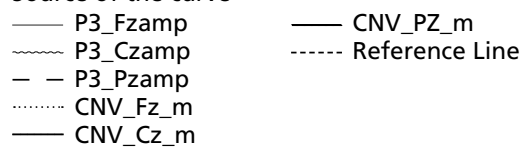

Figure 1. Receiver-operating characteristic curves (ROC) of the comparison between $A D$ and healthy controls. Top left: cognitive tasks (M: mean of two sessions), top right: frequency analysis (legend indicates frequency band and electrode). Bottom left: 40Hz-SSR power and P300 latency (legend indicates paradigm and electrode position), bottom right: P300 amplitude and CNV amplitude (legend indicates paradigm and electrode position). The ROC values are calculates for the mean of the two identical recording sessions. 
the $\mathrm{P} 3$ and $\mathrm{O} 2$ electrodes (i.e. $83 \%$ ), the specificity is moderate for all electrodes presented. The gamma-band power during music listening showed a moderate sensitivity and specificity. The gamma-band power during the story listening task shows moderate to low sensitivity and specificity. During the visual gamma-band task the sensitivity was high for P3 and 01; the sensitivity is moderate for all electrodes.

In the 40-Hz SSR the T5 and T6 electrodes showed a high sensitivity in discriminating between $\mathrm{AD}$ and $\mathrm{MCl}$. The specificity of the $40-\mathrm{Hz}$ SSR was moderate.

In the oddball task the P300 amplitude showed a high sensitivity and a moderate specificity in discriminating the $A D$ patients from controls. The sensitivity of the P300 latency was moderate. The specificity of the P300 latency was moderate to low.

The CNV paradigm showed a moderate sensitivity and moderate to low specificity in discriminating the $A D$ patients from the controls.

\section{Comparison between the $A D$ and $M C l$ group}

Table 2 presents the sensitivity, specificity and AUC of the comparison between the $A D$ and the $M C l$ patients. The ROC curves presented in figure 2 illustrate the sensitivity and specificity of the discrimination between the $A D$ and the $M C I$ group.

The ADAS-cog and MMSE showed a high sensitivity and specificity in discriminating between $A D$ and $\mathrm{MCl}$ patients. The SRT showed a moderate sensitivity and specificity in discriminating between $A D$ and $\mathrm{MCI}$. The CRT showed a very high sensitivity and a moderate specificity in discriminating between $A D$ and $\mathrm{MCl}$.

The delta band during resting state showed a moderate sensitivity and specificity in discriminating between $A D$ and $\mathrm{MCl}$. The theta band during resting state showed a moderate sensitivity and a low specificity in discriminating between $A D$ and $\mathrm{MCl}$. The alpha band during resting state showed a moderate sensitivity and a low specificity in discriminating between $A D$ and $\mathrm{MCl}$. The beta band during resting state showed a moderate sensitivity and a low specificity in discriminating between $\mathrm{AD}$ and $\mathrm{MCl}$.

The gamma-band power during resting state showed a high sensitivity for the P3 electrode and a moderate sensitivity for the other electrodes. The specificity of the gamma-band power during resting state was moderate. The gammaband power during the music listening task showed a moderate sensitivity and specificity in discriminating between $A D$ and $\mathrm{MCl}$ patients. During the visual task there was a high sensitivity for the $\mathrm{P} 3$ and $\mathrm{P} 4$ electrodes. The sensitivity at $\mathrm{P} 4, \mathrm{O} 1$ and $\mathrm{O} 2$ was moderate. The specificity during the visual task was 
Functional and structural brain markers of Alzheimer's disease | Chapter 6

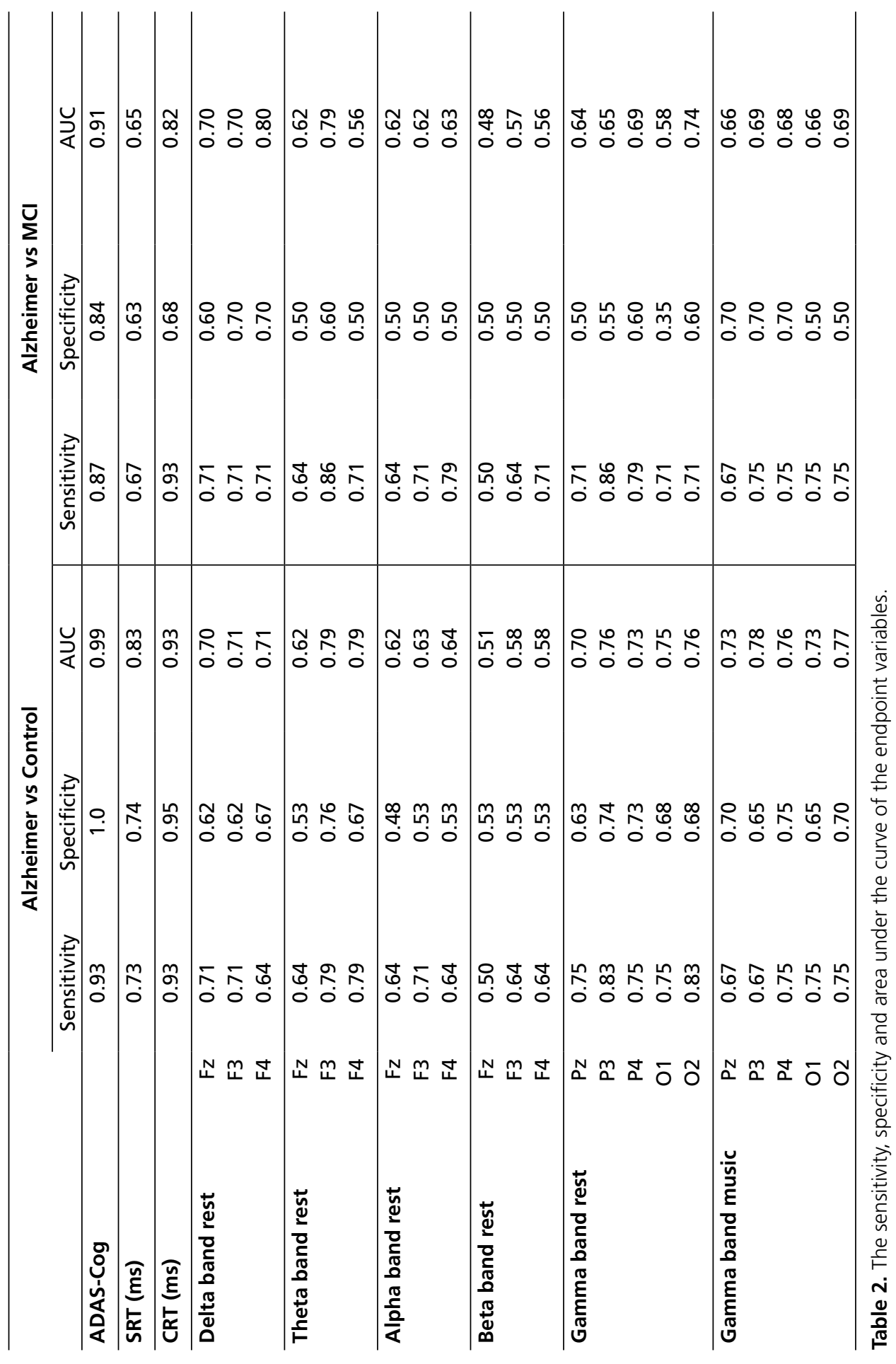




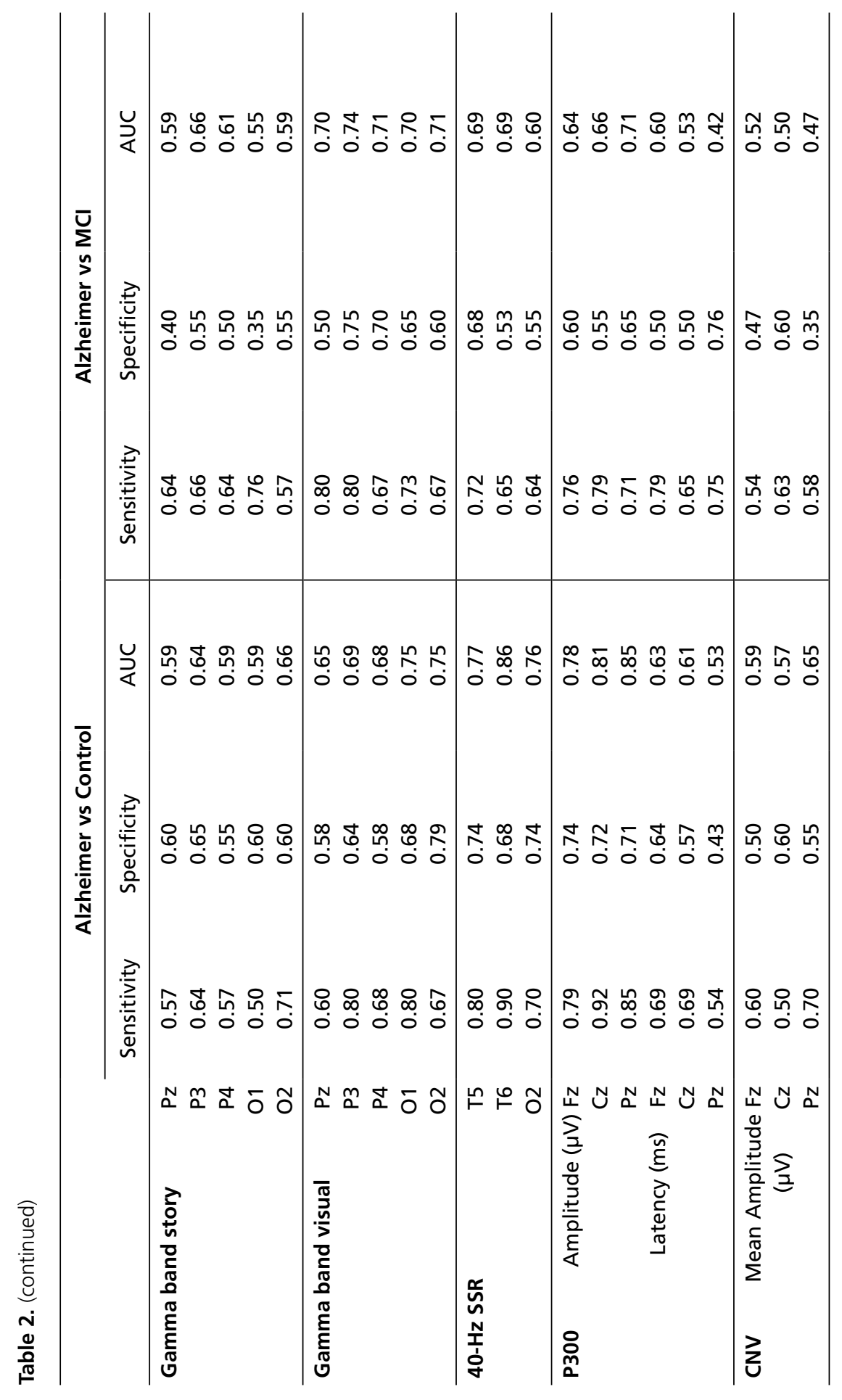


moderate, only Pz showed a low specificity. The gamma-band power during story listening showed a moderate sensitivity in discriminating between $A D$ and $\mathrm{MCl}$. The gamma-band power during story listening was moderate for P3 and P4 and low for the other electrodes.

\section{Discussion}

The current study compared the sensitivity, specificity and reliability of a variety of EEG paradigms and cognitive tasks when it comes to distinguishing AD patients from controls. The response speed tasks, and especially the CRT, proved to be very sensitive, specific and reproducible. Our findings are in line with a previous study, which showed that response speed measured with CRT is very sensitive for the effects of therapeutic intervention (Caramelli et al., 2004). This suggests that response speed may be of interest and deserves more attention as a marker with which to assess the effect of therapeutic interventions.

The present study further showed that the test-retest reliability of the outcome measures was moderate to high, except in the case of the gamma-band power during visual stimulation and the CNV amplitude. The TRR of the frequency analysis as well as the P300 amplitude and latency were in line with findings by previous studies that investigated healthy subjects (Frund et al., 2007; Polich and Herbst, 2000; Williams et al., 2005). The TRR of the 40-Hz SSR was moderate to high in the $A D$ and $\mathrm{MCl}$ groups, but was relatively low in the control group. These findings suggest that the $40-\mathrm{Hz}$ SSR can reliably be used in an elderly cognitively impaired population. The CNV paradigm showed a moderate TRR in the $\mathrm{MCl}$ and control groups but a very low TRR in the AD group. The present results suggest that the CNV cannot reliably be used in an AD population. ADAS.

Of all the measures included in this study, ADAS-cog proved to be the most sensitive, specific and reliable in discriminating $A D$ from $\mathrm{MCl}$ and healthy controls. The finding that ADAS-cog was the most sensitive and specific measure is not surprising, since in the current study the clinical diagnosis was used as the gold standard. The clinical diagnosis relies for a part on an extensive neuropsychological evaluation. Although the ADAS-cog is not included as a diagnostic instrument, there is a substantial overlap in the cognitive domains, which are assessed by it.

When we consider the EEG paradigms it becomes clear that in discriminating between $A D$ and healthy controls, the gamma-band power during resting state and music listening, the $40-\mathrm{Hz}$ SSR and the P300 amplitude showed the 

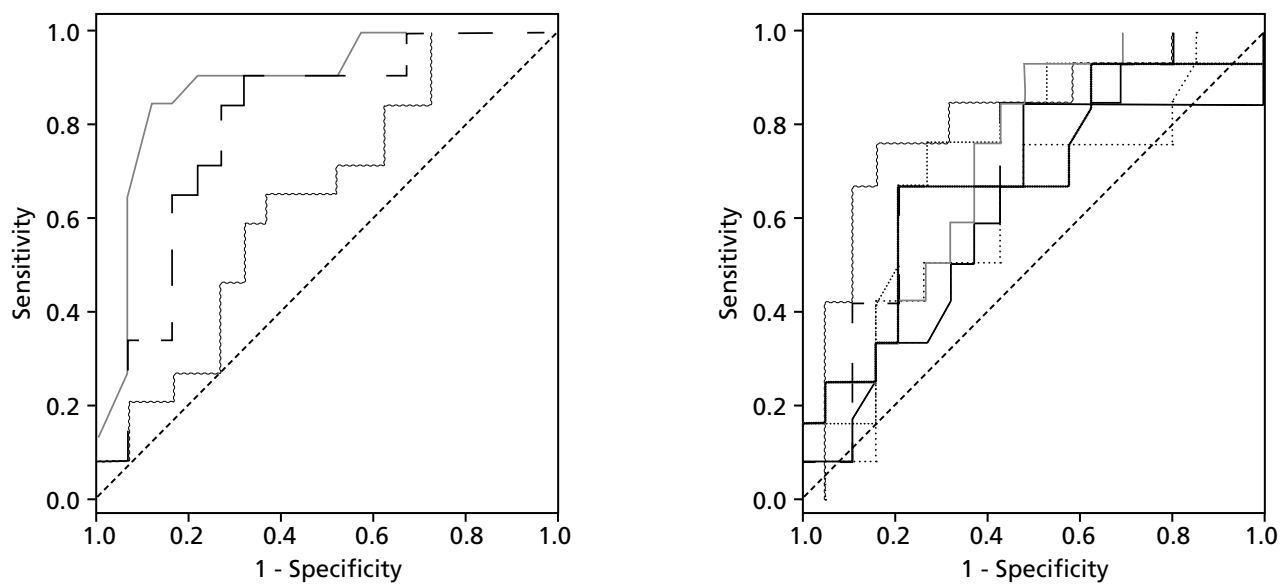

Source of the curve

- ADAS_M

SRT_M

- - CRT M

-.---. Reference Line

Source of the curve

- Rest_delta_F3 Music_gamma_P3

mest_theta_F3

- - Rest_alpha_F3

Rest_beta_F3
Rest_gamma_P3

- Music_gamma_P3

........... Visual_gamma_P3

-..--- Reference Line
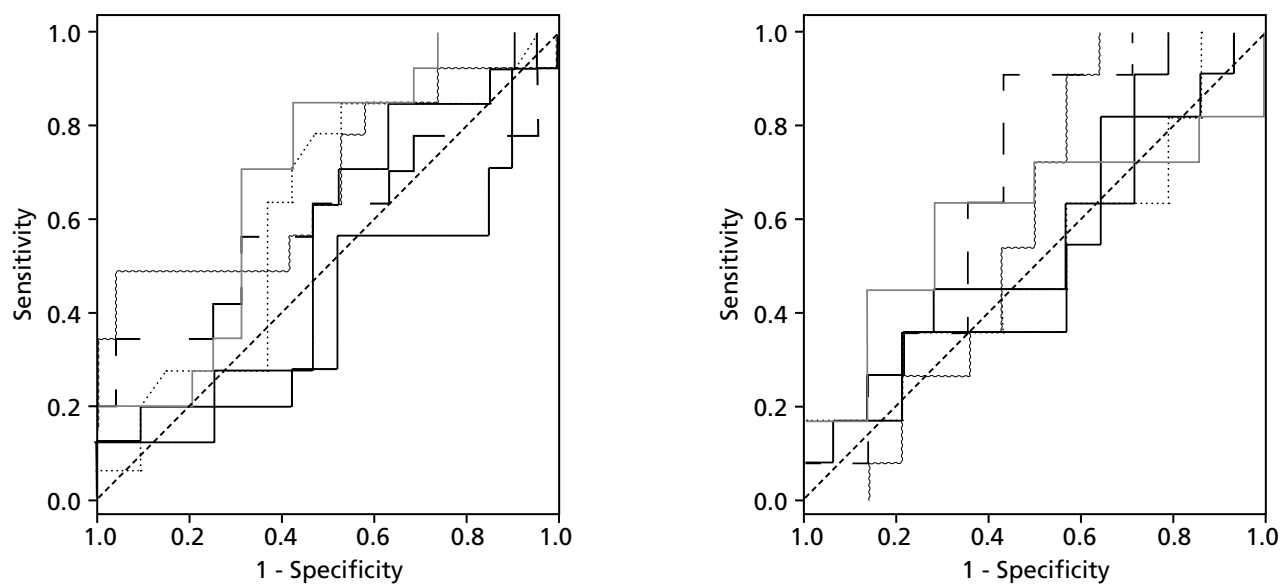

Source of the curve

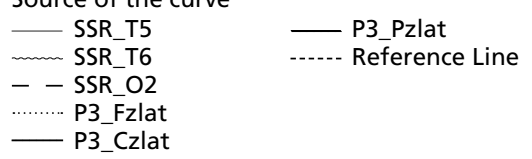

Figure 2. Receiver-operating characteristic curves (ROC) of the comparison between $A D$ and $\mathrm{MCl}$. Top left: cognitive tasks (M: mean of two sessions), top right: frequency analysis (legend indicates frequency band and electrode). Bottom left: $40 \mathrm{~Hz}$-SSR power and P300 latency (legend indicates paradigm and electrode position), bottom right: P300 amplitude and CNV amplitude (legend indicates paradigm and electrode position). The ROC values are calculates for the mean of the two identical test sessions. 
best sensitivity and specificity. The sensitivities, specificities and AUCs of these paradigms were comparable to each other. When it comes to discriminating between $A D$ and $\mathrm{MCl}$, the gamma-band power measured during music listening and during the visual task, and the delta-band power measured during resting state showed the highest sensitivity and specificity. The specificity of the theta and alpha band was lower than in previous studies (Jelic et al., 1996). This might be related to the fact that in the present study EEG was measured with the subject's eyes open. EEG measurement with open eyes attenuates the power of the theta and especially of the alpha band.

In sum, the present study showed that several EEG paradigms are sensitive, specific and reliable in discriminating $A D$ from $\mathrm{MCl}$ and healthy controls. Nevertheless, the diagnostic accuracy of the ADAS-cog and the CRT exceed that of the EEG paradigms. Several studies reported a clear relation between the severity of $A D$ and changes in the low-frequency range (Kowalski et al., 2001), the gamma band (van Deursen et al., 2008), and the P300 components (Bennys et al., 2007). However, the lower sensitivities and specificities suggest that the EEG paradigms studied here will not have an additional value in the diagnosis of $A D$.

Also when it comes to the staging of $A D$, clinical parameters, such as the global deterioration scale (GDS) (Reisberg et al., 1982) probably provide more accurate and useful information than EEG paradigms. Another disadvantage of the use of EEG as a diagnostic instrument is that there are no standard norms to which a patient's 'performance' can be compared.

Besides diagnostic accuracy another important aim of this study was to determine whether EEG is useful as a marker to evaluate treatment interventions. Although the present study did not evaluate this aspect in itself, an important characteristic of such markers is their ability to discriminate between AD patients, $\mathrm{MCl}$ patients and healthy control subjects. Several studies showed that cholinesterase inhibitors have an effect on the EEG frequency and also on the amplitude and latency of the ERPs. Previous studies showed that changes in the lower frequencies are sensitive to the effects of cholinergic treatments (Rodriguez et al., 2002; Sneddon et al., 2006). The gamma-band paradigms presented in the current study have not yet been used to evaluate the effect of treatment interventions. Although several studies have reported that cholinergic deafferentiation and synaptic blockade have a profound effect on gammaband coherence (Holschneider et al., 1999; Rodriguez et al., 2004), the sensitivity of gamma-band changes to therapeutic interventions requires further research.

The present study further showed that when it is used as a diagnostic instrument, the P300 amplitude is more sensitive, specific and reliable in discriminating between patients groups. In contrast, studies that assess the effect of 
treatment interventions indicate that the P300 latency seems to be more sensitive (Katada et al., 2003; Werber et al., 2003). Both these findings showed that the treatment related changes in P300 latencies were associated significantly with changes in the ADAS-cog. This suggests that the P300 complex, and latency in particular, could be complementary to ADAS-cog in the study of cholinergic treatment effects.

At present, the sensitivity of the $40-\mathrm{Hz}$ SSR as a marker for therapeutic effects has not been studied. Nevertheless changes in the $40-\mathrm{Hz}$ SSR in healthy populations have proved to be very sensitive for manipulations of the cholinergic nervous system (Ahveninen et al., 2002; Ahveninen et al., 1999). These findings suggest that the $40-\mathrm{Hz}$ SSR might be an interesting marker in the evaluation of therapeutic effects, but this will need to be substantiated by future research.

Of the EEG paradigms that are presented here, only the slow frequencies and the P300 complex have actually been used in the follow-up of cholinergic treatments. Both changes in the slow EEG waves and in the P300 showed that the therapy induced EEG changes which correlated significantly with the improvement of cognitive performance (Katada et al., 2003; Rodriguez et al., 2002; Werber et al., 2003). This association between cognitive performance and changes in the EEG is an important one, since it demonstrates that the cognitive measures and the EEG changes measure the same process, but at a very different level. This suggests that the changes in the EEG frequency and in the P300 complex, and in latency in particular, could be used complementary to cognitive assessment scales in the study of treatment effects.

Response speed tasks and EEG paradigms could probably have an additional value for clinical rating scales such as the ADAS-cog, since these measures are not biased by motivational aspects of the patients and subjective interpretations by the rater. Further research is required to investigate whether EEG or response speed tasks are able to increase the sensitivity to therapeutic interventions in longitudinal studies.

A strong aspect of the current study was that different EEG paradigms were compared head to head in a well-defined, psychoactive drug-naïve population. A disadvantage of the present study was that conclusions regarding specificity were limited to $A D, M C l$ and healthy controls and that other forms of dementia, such as vascular dementia and frontotemporal dementia, were not included. Another disadvantage of this study was its relatively small sample size.

Based on the present findings we can conclude that EEG paradigms have no additional value in the diagnosis of $A D$. However, in the evaluation of treatment interventions EEG does merit more attention as a complementary outcome measure. 




$$
\text { Chapter }
$$





\title{
Neuroanatomical correlates of neuropsychiatric syndromes in Alzheimer's disease
}

\author{
Submitted for publication \\ Jeroen A. van Deursen, Frans R. Verhey, Paul A. Hofman, Frederik Barkof, Pauline Aalten
}

\begin{abstract}
Neuropsychiatric symptoms are a common feature of Alzheimer's disease and have a negative effect on the quality of life of patients and their caregivers. The pathogenesis or neural basis of neuropsychiatric symptoms in AD has not yet been clarified however. Studies using neuroimaging of neuropsychiatric symptoms in AD have shown a large variability in the brain areas associated with individual symptoms. The accumulating evidence that neuropsychiatric symptoms rarely occur in isolation, but more often in syndromes, points to a common pathogenesis.

The aim of the present study was to investigate the association between neuropsychiatric syndromes and cortical volume differences using voxelbased morphometry (VBM). The neuropsychiatric inventory (NPI) was used to assess the presence and severity of symptoms, which were grouped in four syndromes, viz.: hyperactivity, psychosis, affective disorders and apathy. The results show that hyperactivity is associated with decreased gray matter (GM) densities in the bilateral parahippocampal cortex; in the left middle temporal gyrus; in the bilateral medial frontal gyrus; and in the anterior cingulate cortex. Psychosis was associated with decreased GM densities in the left
\end{abstract}


medial frontal gyrus; in the right anterior cingulate; in the right caudate nucleus; and in the left middle temporal gyrus.

The present study is the first to investigate the volumetric correlates of neuropsychiatric syndromes in AD. Future studies should be able to point out whether neuroimaging of the neuropsychiatric syndromes in AD provides more consistent results than can be obtained by focusing on individual symptoms.

\section{Introduction}

Although memory deficits and other cognitive impairments are considered the major hallmarks of Alzheimer's disease (AD), neuropsychiatric symptoms are prevalent at some moment during the disease in over $95 \%$ of the patients (Aalten et al., 2005a; Steinberg et al., 2006; Steinberg et al., 2003). Neuropsychiatric symptoms in $A D$ have a negative effect on the quality of life of the patient (Shin et al., 2005); they increase the caregiver's burden (de Vugt et al., 2006); they are important risk factors for early hospitalization (Tun et al., 2007); and they increase healthcare costs (Jonsson et al., 2006). Reduction of the frequency and the severity of neuropsychiatric symptoms in patients with $A D$ is therefore important from a healthcare as well as a health economics perspective.

Individual neuropsychiatric symptoms rarely occur in isolation and associations between different symptoms have been shown to exist (Aalten et al., 2007b; Frisoni et al., 1999; Lyketsos et al., 2001). The pathogenesis or neural basis of neuropsychiatric symptoms in $A D$ has not yet been clarified however. Several studies have used neuroimaging techniques to gain more insight into its pathogenesis and have reported associations between the presence of neuropsychiatric symptoms and regional, structural and functional changes. Apathy for example, one of the most common neuropsychiatric disorders in $A D$, has been associated with hypo-perfusion and tissue loss in the anterior cingulate cortex, inferior temporal cortex, frontal cortex and lentiform nucleus (Benoit et al., 2002; Bruen et al., 2008a; Migneco et al., 2001; Robert et al., 2006b; Rosen et al., 2005).

Furthermore, a volumetric study by Bruen et al. (2008) showed associations between delusions and the bilateral inferior frontal cortex. This study revealed an association between agitation and the bilateral anterior cingulate cortex and the left insula in an AD population. Another study by Rosen et al. (2005) showed volumetric differences associated with disinhibition and agitation in the subgenual cingulate gyrus, as well as the anterior cingulate cortex and left insula. Both these studies by Bruen at al. (2008) and Rosen et al. (2006) 
examined gray matter volume changes using voxel-based morphometry (VBM). VBM is a technique for assessing atrophy of the whole brain in an automated manner. Traditionally, changes in regional brain volumes are assessed with a region of interest (ROI) approach. This approach has drawbacks however as it is very time consuming and restricted by the number of brain areas that are defined. VBM is better suited to the task as it does not need a-priori assumptions about the location of potential volumetric differences (Ashburner and Friston, 2000). This makes VBM an ideal method for studying the association between the presence of neuropsychiatric syndromes and cortical volume changes.

Both the VMB studies by Rosen et al. (2005) and Bruen et al. (2008) mentioned above, focused on the association between gray matter density and individual neuropsychiatric symptoms. These studies discovered that different brain areas were associated with different neuropsychiatric symptoms. Recent evidence suggests that the use of neuropsychiatric syndromes instead of isolated symptoms might provide a better approach for the study of the neural fundaments of neuropsychiatric disorders in AD. Moreover, a recent study by the European Alzheimer's Disease Consortium (EADC) has provided evidence for the presence of four major neuropsychiatric syndromes, viz.: hyperactivity, psychosis, affective disorders and apathy (Aalten et al., 2008; Aalten et al., 2007b). The identification of neuropsychiatric syndromes points to a common neurobiological pathogenesis. Since all previous neuroimaging studies focused on individual symptoms, the existence of a common pathogenesis or neural basis for neuropsychiatric syndromes in AD has not yet been clarified.

Therefore, the current study aims to investigate the association between neuropsychiatric syndromes and cortical volume differences with the help of VBM.

\section{Materials and Methods}

\section{Subjects}

Thirty-seven patients with a diagnosis of probable Alzheimer's Disease according to the DSM-IV and NINCDS-ADRDA criteria (McKhann et al., 1984) were included in this study. Standard blood work and neuroimaging (CT or MRI) were carried out and the diagnosis was supported by abnormal performance on neuropsychological tests. The main exclusion criteria were: a history of stroke; head trauma; and/or any other neurological or psychiatric disorders. Additional exclusion criteria were: severe cardiovascular disease: and/or a history of substance abuse; and/or other serious system diseases (e.g. malignancy, uncontrolled hypertension, neuropathy or seizure disorders). All AD patients were recruited at the 
Memory Clinic of the Maastricht University Medical Centre; they were not institutionalized and had a reliable informant. All the patients and a primary caregiver gave written informed consent prior to the study. The study was carried out in accordance with the declaration of Helsinki. The local Medical Ethics Committee of the Maastricht University Medical Centre approved the study.

\section{Neuropsychiatric assessment}

The neuropsychiatric test battery consisted of the Neuropsychiatric Inventory (NPI), which is a semi-structured instrument for assessing the presence and severity of 12 neuropsychiatric symptoms (Cummings, 1997; Cummings et al., 1994). The frequency and severity of each symptom are scored on the basis of structured questions administered to the patient's primary caregiver. The continuous score for each symptom is obtained by multiplying severity (1-3) by frequency (1-4). The content validity, concurrent validity, inter-rater reliability and test-retest reliability of the original English NPI have been established (Cummings and McPherson, 2001). In line with previous studies, a score greater than 3 was taken to indicate the presence of 'clinically relevant' symptoms (Aalten et al., 2005a; Ballard et al., 2001; Lyketsos et al., 2002; Steinberg et al., 2003; Steinberg et al., 2004). In the current study the Dutch validated version of the NPI was used (Kat et al., 2002b). The results of the NPI were assessed prior to MRI acquisition and were therefore scored independently without prior knowledge of the atrophy status of the patient's brain.

For the present study, neuropsychiatric syndromes were used in accordance with the results of the EADC study, which are extensively described in Aalten et al. (Aalten et al., 2007b). The four syndromes were calculated by adding up the accompanying NPI symptom scores as follows: 1 ) hyperactivity (agitation + disinhibition + irritability + aberrant motor behavior + euphoria); 2) psychosis (delusions + hallucinations + nighttime behavior); 3 ) affective disorders (depression + anxiety); 4) apathy (apathy + eating abnormalities). The syndromes were considered clinically significant when one or more of the accompanying symptoms scored higher than 3 on the NPI.

\section{Neuropsychological assessment}

All subjects underwent a neuropsychological test battery to measure their cognitive functioning consisting of the Cambridge Examination for Mental Disorders of the Elderly part B (CAMCOG) (Derix et al., 1991; Roth et al., 1986), which incorporates the Mini-Mental State Examination (MMSE) (Folstein et al., 1975a). Sub-domains in the CAMCOG are: attention, orientation, language comprehension, expression, memory, praxis, abstract reasoning and perception. The 
neuropsychological evaluation further included: the Global Deterioration Scale (GDS) (Reisberg et al., 1982); Fluency (Lezak, 1995b); the Stroop Color Word Task (STROOP) (Houx et al., 1993); the ten-word learning task (10-WLT), which is derived from the auditory verbal learning task (Brand and Jolles, 1985); the Concept Shifting task (CST) (Vink and Jolles, 1985b); and the Letter-digit-symbol task (LDST) (Lezak, 1995). A neuropsychological evaluation was available for 35 of the participants.

\begin{tabular}{|c|c|c|c|}
\hline Symptom NPI & Mean and SD & $\begin{array}{l}\text { Patie } \\
\text { sym } \\
\text { score } \\
\%\end{array}$ & $\begin{array}{l}\text { with } \\
\text { /syndromes } \\
\text { N }\end{array}$ \\
\hline Delusions & $0.76 \pm 2.22$ & 10.8 & 4 \\
\hline Hallucinations & $0.05 \pm 0.33$ & 0.0 & 0 \\
\hline Agitation & $1.54 \pm 3.11$ & 13.5 & 5 \\
\hline Depression & $1.81 \pm 2.53$ & 24.3 & 9 \\
\hline Anxiety & $0.92 \pm 1.89$ & 8.1 & 3 \\
\hline Elation & $0.49 \pm 1.35$ & 8.1 & 3 \\
\hline Apathy & $2.62 \pm 2.91$ & 32.4 & 12 \\
\hline Disinhibition & $0.22 \pm 0.58$ & 0.0 & 0 \\
\hline Irritability & $1.54 \pm 2.97$ & 16.2 & 6 \\
\hline Aberrant motor behavior & $1.97 \pm 2.91$ & 29.7 & 11 \\
\hline Sleep disorders & $0.62 \pm 1.67$ & 8.1 & 3 \\
\hline Eating disorders & $1.43 \pm 2.66$ & 21.6 & 8 \\
\hline Hyperactivity syndrome & $5.76 \pm 7.68$ & 48.6 & 17 \\
\hline Psychosis syndrome & $1.43 \pm 3.67$ & 16.2 & 6 \\
\hline Affective disorders syndrome & $2.73 \pm 3.71$ & 27.0 & 10 \\
\hline Apathy syndrome & $4.05 \pm 4.28$ & 45.9 & 17 \\
\hline
\end{tabular}

Table 1. Percentage, mean frequency*severity and standard deviation for each symptom and syndrome on the NPI.

\section{Structural MRI: scanning and analysis}

A 3.0 Tesla Gyroscan NT MRI scanner (Phillips ${ }^{\circledR}$ ) was used to obtain the following MRI scans: 3D-ISO T1W FFE, T2W-TSE, T2W-IR FLAIR, and IR-TSE scans. A coiled gradient was used, which provided high anatomical resolution and good grey/ white matter contrast for subsequent segmentation and VBM. The T1-weighted 3D-ISO scan was used for VBM analysis and was acquired using the following 
parameters: voxel size $=1 \times 1 \times 1 \mathrm{~mm}$; TR $=8.1 \mathrm{~ms}$; TE $=3.7 \mathrm{~ms}$; TFE $=230 \mathrm{~ms}$; flip angle $=8^{\circ} ;$ matrix $=224 \times 224$ pixels; FOV $=224 \times 224 \mathrm{~cm}^{2}$.

Image preprocessing was performed using Statistical Parametric Mapping (SPM 5) (Wellcome Trust Centre for Neuroimaging). The first step was a 12-parameter affine registration with the MNI template as a target. We chose not to perform a non-linear registration procedure, because a previous study of $A D$ patients showed mainly expansions/contractions in the lateral ventricles and minimal changes in the gyri and sulci after this procedure (Karas et al., 2008a). The registered images were segmented into gray matter (GM), white matter (WM) and CSF probability maps, using the SPM 5 segmentation priors. Registration accuracy of the gray matter probability maps was further enhanced by a registration method that uses alignment and scaling to spread the registration bias among the whole group. The transformation matrix is averaged by projection to a manifold, a method described in more detail in Karas et al. and Woods et al. (Karas et al., 2004; Woods, 2003). The registered gray matter volumes were smoothed using a Gaussian kernel filter set at $12 \mathrm{~mm}$ (full with half maximum) to remove individual variations of gyral anatomy.

The smoothed GM images were entered in a multiple regression model, where their linear correlation with NPI syndromes was calculated. Age and MMSE were entered in this model as covariates for all variables. The $x, y, z$ coordinates of the areas with a significant correlation between GM density and scores on the neuropsychiatric syndromes were identified using the Talairach Deamon Client tool (www.talairach.org). An image of the average GM volume of the current population was used to map the significant results.

\section{Statistical analysis}

Statistical analysis of the behavioral data was performed using the Statistical Package for Social Sciences (SPSS), version 16.0. Descriptive statistics were used to examine the presence and severity of the neuropsychiatric syndromes. The correlation between the neuropsychiatric syndromes and the neuropsychological results were calculated using Pearson's correlation coefficients.

\section{Results}

\section{Demographics and neuropsychiatric evaluation}

The participating patients in this study had a mean age of $72.6( \pm 9.1)$; mean MMSE was $20.4( \pm 4.1)$; and mean GDS was $3.5( \pm 0.9)$. The GDS and MMSE scores show that this was a mild to moderate AD population. Twenty-five subjects were 


\begin{tabular}{lcccc}
\hline Brain Area & Left/Right & $\begin{array}{c}\text { Brodmann } \\
\text { area }\end{array}$ & $\begin{array}{c}\text { MNI } \\
\text { Coordinate }\end{array}$ & $\begin{array}{c}\text { Z-score at } \\
\text { maximum }\end{array}$ \\
\hline Hyperactivity & Left & 35 & $-16-23-27$ & 3.57 \\
Parahippocampal gyrus & Left & 31 & $-503-27$ & 3.42 \\
Middle temporal gyrus & Right & 36 & $24-22-33$ & 3.46 \\
Parahippocampal gyrus & Left & 11 & $-1024-12$ & 3.32 \\
Medial frontal gyrus & Right & 10 & $1047-4$ & 2.76 \\
& Right & 32 & $623-10$ & 3.15 \\
Anterior cingulate cortex & & & & \\
\hline Psychosis & Left & 11 & $-1027-10$ & 3.93 \\
Medial frontal gyrus & Right & 32 & $823-8$ & 3.61 \\
Anterior cingulate cortex & Right & & $610-6$ & 3.93 \\
Caudate nucleus head & Left & 21 & $-5011-34$ & 3.30 \\
Middle temporal gyrus & Le & & & \\
\hline
\end{tabular}

Table 2. Brain regions with a significant correlation between GM density and scores on hyperactivity and psychosis.

male and twelve were female. In the present population thirty patients were on cholinesterase inhibitor treatment; one patient was on memantine treatment; eight patients used antidepressants; one patient used a benzondiazepine; and one patient used a neurolepticum.

The descriptive variables of the neuropsychiatric evaluation are presented in table 1. Apathy was the most frequently identified neuropsychiatric symptom (32.4\%), while clinically significant hallucinations and disinhibition were absent. Hyperactivity (48.6\%) and apathy (48.6\%) were the most frequently identified syndromes, while affective disorders (27.0\%) and psychosis (16.2\%) were less prevalent in the current population.

The correlations of the patient characteristics and neuropsychological variables with the neuropsychiatric syndromes showed that increasing age was associated with higher scores on the affective syndrome $(r=0.38 p=.019)$.

The hyperactivity syndrome was associated with higher levels of cognitive impairment as measured by MMSE $(r=0.40 p=.013)$ and CAMCOG $(r=0.38$ $p=.023)$. The hyperactivity syndrome was further associated with impaired performance in the CAMCOG sub-domains related to attention $(r=0.34 p=.049)$ and language expression $(r=0.46 p=.005)$.

The psychosis syndrome was associated with impaired cognitive performance as measured by MMSE $(r=0.43 p=.05)$, CAMCOG $(r=0.50 p=.003)$, GDS $(r=0.38 p=.020)$ and verbal fluency $(r=0.37 p=.029)$. The psychosis syndrome was further associated with impaired performance in the CAMCOG sub- 
domains related to orientation $(r=0.40 p=.019)$, language expression $(r=0.63$ $p<.000)$ and abstract reasoning $(r=0.47 p=.004)$.

The apathy syndrome showed no significant correlation with neuropsychological variables or demographic attributes.

\section{Voxel-based morphometry}

As shown in table 2, the voxel-based multiple regressions showed significant correlations between GM densities and the neuropsychiatric syndromes of hyperactivity and psychosis. High scores on the hyperactivity syndrome correlated significantly with decreased GM densities in the bilateral temporal lobes and in the left medial and right anterior cingulate cortex (see figure 1). High scores on the psychosis syndrome correlated significantly with decreased GM densities in the left medial frontal gyrus, in the left middle temporal gyrus, and in the head of the right caudate nucleus and left anterior cingulate cortex (see figure 2).

The affective disorders and apathy syndromes did not show any significant correlations with GM densities.

\section{Discussion}

The primary aim of the current study was to determine the neuro-anatomical correlates of neuropsychiatric syndromes in AD. The present study showed that the neuropsychiatric syndromes of hyperactivity and psychosis were significantly associated with GM tissue loss.

The hyperactivity syndrome was associated with GM tissue loss in the bilateral parahippocampal gyri; in the left medial temporal gyrus; in the bilateral medial frontal gyrus; and in the right anterior cingulate cortex. Reduced GM densities had previously been shown in the anterior cingulate cortex in association with aberrant motor behavior and agitation in AD (Bruen et al., 2008a; Rosen et al., 2005). These VBM findings are in line with a study that showed that a high neurofibrillary tangle (NFT) load in these areas is associated with agitation and aberrant motor behavior (Tekin et al., 2001).

Reduced GM density in the anterior cingulate cortex and medial frontal gyrus have previously been related to disinhibition (Rosen et al., 2005). The parahippocampal gyri and anterior cingulate cortex are part of the limbic system, which is involved in emotional processing and inhibition. Lesions in the limbic system are closely related to agitation, irritability and social disinhibition (Tekin and Cummings, 2002). A recent FMRI study showed that hypersensitivity of the amygdala in $A D$ patients correlated with aggression/agitation and 
A

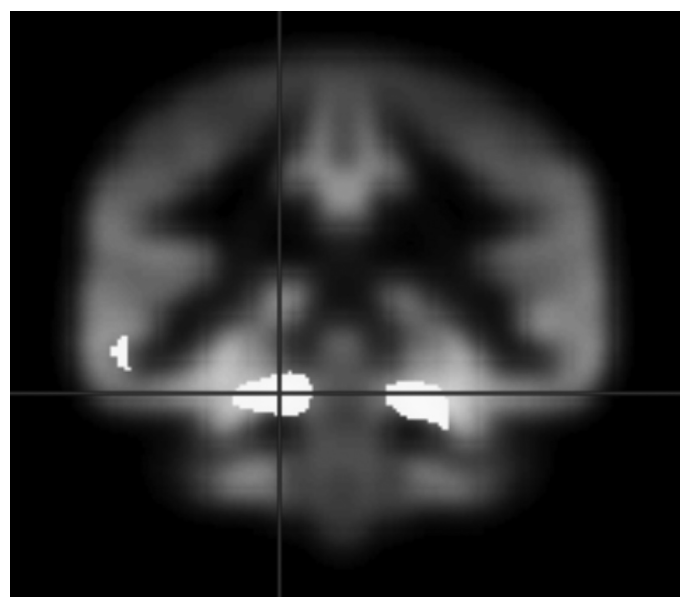

B

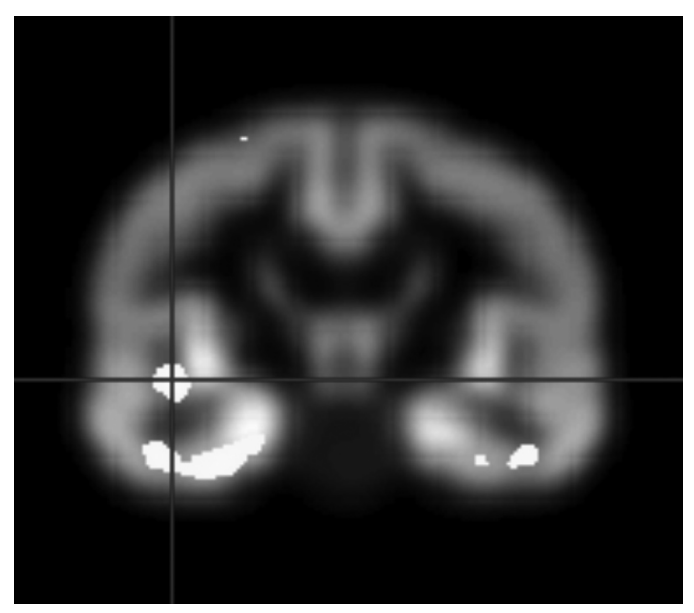

Figure 1. Brain areas with a significant correlation with the hyperactivity syndrome: (A) left parahippocampal gyrus; (B) left medial temporal gyrus.

irritability symptoms (Wright et al., 2007). Although the current study did not yield any volume differences in the amygdala, the disruption of the connections within the limbic system might explain the presence of hyperactive behavior in AD (Tekin and Cummings, 2002).

The psychosis syndrome was associated with decreased GM densities in the left medial frontal and the medial temporal gyri; in the right anterior cingulate cortex; and in the head of the right caudate nucleus. An earlier VBM study showed that decreased GM densities in the left medial frontal cortex are associated with delusions (Bruen et al., 2008a). In studies examining regional cerebral blood flow ( $\mathrm{CCBF}$ ), hypoperfusion of the right frontal and temporal 
cortices seems to be associated with delusions and hallucinations in AD (Lopez et al., 2001a; Nakano et al., 2006; Staff et al., 1999).

In the present study we discovered decreased GM densities in caudate nucleus and anterior cingulate cortex in the right hemisphere. The caudate nucleus and anterior cingulate cortex are closely interconnected and usually involved in motivated behavior and executive functions (Tekin and Cummings, 2002). The relation of these areas to psychosis in $A D$ is not clear yet, but reduced $r C B F$ has been shown in the anterior cingulate cortex in $A D$ patients with psychotic symptoms (Lopez et al., 2001b).

The current study further revealed decreased GM density in the left medial temporal lobe, a finding that is in accordance with the decreased $\mathrm{rCBF}$ that has been shown before with regard to psychotic symptoms in AD (Lopez et al., 2001a). There is evidence that the load of neurofibrillary tangles in the middle frontal cortex, temporal cortex and basal brain structures are closely connected to psychotic symptoms in AD (Farber et al., 2000). The presence of neurofibrillary tangles is closely associated with increased rates of local atrophy (Whitwell et al., 2008). The present findings are in line with previous studies, which suggest that psychosis in AD is associated with dysfunction of the frontal cortex, the temporal cortex and the basal forebrain.

The present study further showed that the hyperactivity and psychosis syndrome were associated with impaired general cognitive performance. These findings, together with the relation between disease severity as measured by GDS and psychosis, are in line with previous findings (Aalten et al., 2005b). The psychosis syndrome was associated with impaired performance in orientation, abstract reasoning, language expression and verbal fluency. The associations between psychosis and language function and abstract reasoning are in line with pervious findings (Aalten et al., 2007a). The impairment of language functions in psychotic $A D$ patients might be associated with the increased rate of atrophy in the left temporal lobe. A previous VBM study showed that impaired word naming is associated with atrophy of the left temporal cortex (Gee et al., 2003).

The association between psychosis and impaired abstract reasoning in the present study is in line with Aalten et al. (2007b). Since abstract reasoning is generally considered a frontal lobe process, atrophy in this area associated with psychosis can explain the relation between abstract reasoning and psychosis. Although the correlations between the neuropsychiatric syndromes and the neuropsychological evaluation were not very strong, they are in line with previous findings and can be explained by atrophy as measured with VBM. This association between behavioral deficits, neuropsychological impairment and volumetric cortical changes, underline the validity of the VBM results. 
A
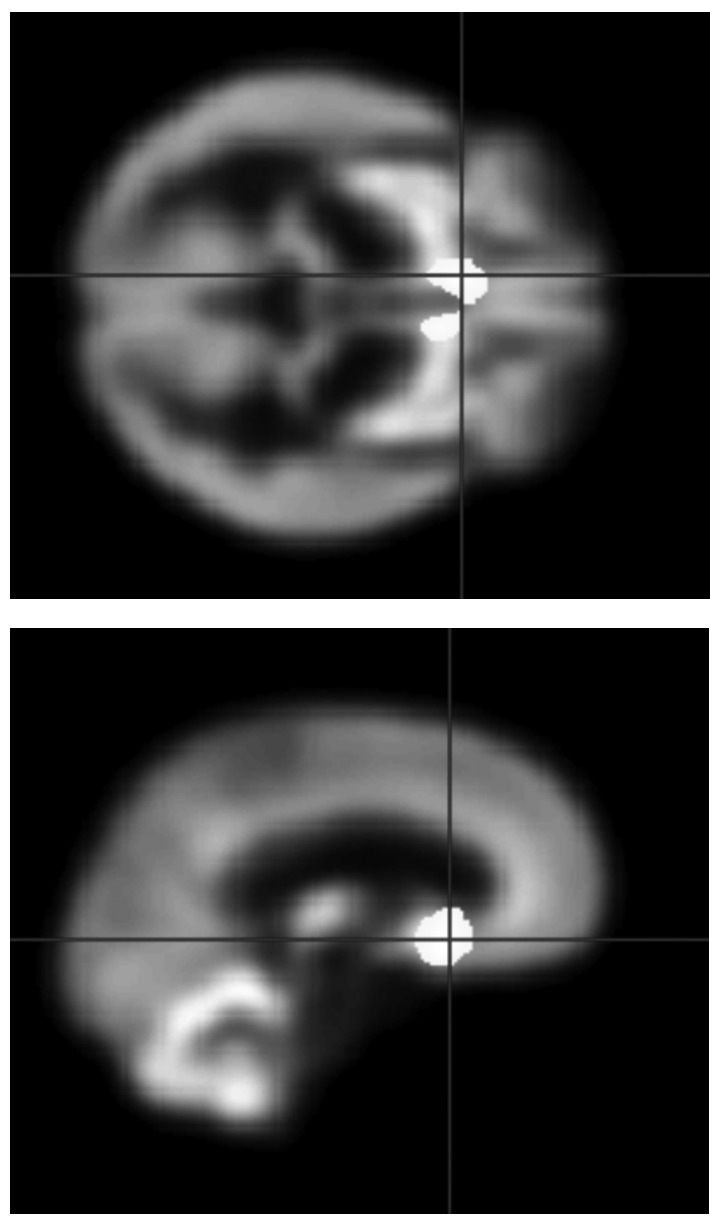

B

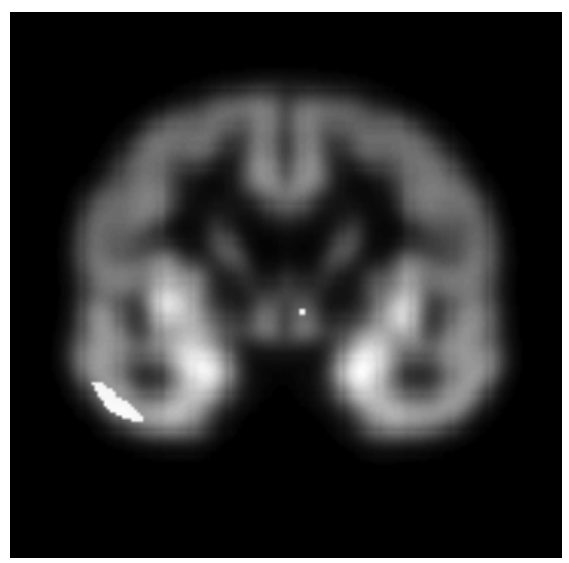

C

Figure 2. Brain areas with a significant correlation with the psychosis syndrome: (A) left medial frontal gyrus; (B) left medial frontal gyrus; (C) left middle temporal gyrus. 
Unexpectedly, no significant associations were found between GM densities and the apathy syndrome. Based on earlier structural and functional neuroimaging research we expected to find an association between apathy and loss of GM volume in the anterior cingulate cortex and in the medial frontal cortex (Benoit et al., 2004; Bruen et al., 2008a; Rosen et al., 2005). The absence of this association might be related to the relatively low frequency of clinically significant apathy, which in the current study was lower (i.e. $32.4 \%$ ) than in most other studies (i.e. $65-80 \%$ ). In the present study the apathy syndrome included the NPI scores of apathy and eating disorders. To ensure that eating disorders did not mask any apathy-associated correlations, a post-hoc analysis was performed in which only the apathy symptom was analyzed. This post-hoc analysis did not reveal a significant association between apathy and GM densities. In future research it would be interesting to study the neural correlate of apathy with more specific ratings scales, such as the Apathy Evaluation Scale (AES) (Marin et al., 1991) and the Apathy Inventory (AI) (Robert et al., 2002).

The current study showed no associations between GM densities and affective disorders. No previous volumetric studies indicating such associations exist, although previous $\mathrm{rCBF}$ studies have shown a relation between depressive symptoms and decreased perfusion (Levy-Cooperman et al., 2008) and metabolism (Holthoff et al., 2005) in the inferior and superior frontal lobe.

Previous neuroimaging studies revealed variability in the exact areas in the brain that have been involved in neuropsychiatric syndromes in AD. This variability is probably related to the different imaging techniques used (i.e. SPECT, PET and VBM); to sample size difference; to different neuropsychiatric rating scales; and to severity of AD. An advantage of the use of VBM is its spatial resolution, which exceeds that of SPECT and PET studies.

The present study was limited by the relatively small sample size, and the use of psychoactive medication. Its strength lies in the fact the MR images were acquired using a 3-T scanner, which increases segmentation accuracy. Another important strength of the current study is that it focused on neuropsychiatric syndromes instead of on symptoms in a well-defined AD population. Neuropsychiatric symptoms rarely occur in isolation; therefore syndromes are probably a better representation of the AD population. Insight into the pathogenesis of neuropsychiatric syndromes is important for the development of effective treatment strategies. Several papers already suggested that a syndromatic approach is more useful when pharmacotherapies are evaluated (Gauthier et al., 2005; Herrmann et al., 2005). Future studies should be able to point out whether neuroimaging of the neuropsychiatric syndromes in AD provides more consistent results than can be obtained by focusing on individual symptoms. 


\section{Funding}

This work was supported by the Dutch Hersenstichting [grant number 12F04.39].

\section{Acknowledgement}

We would like to thank Marc Geerlings and Laura Schellekens for their help in data acquisition and analyses. 



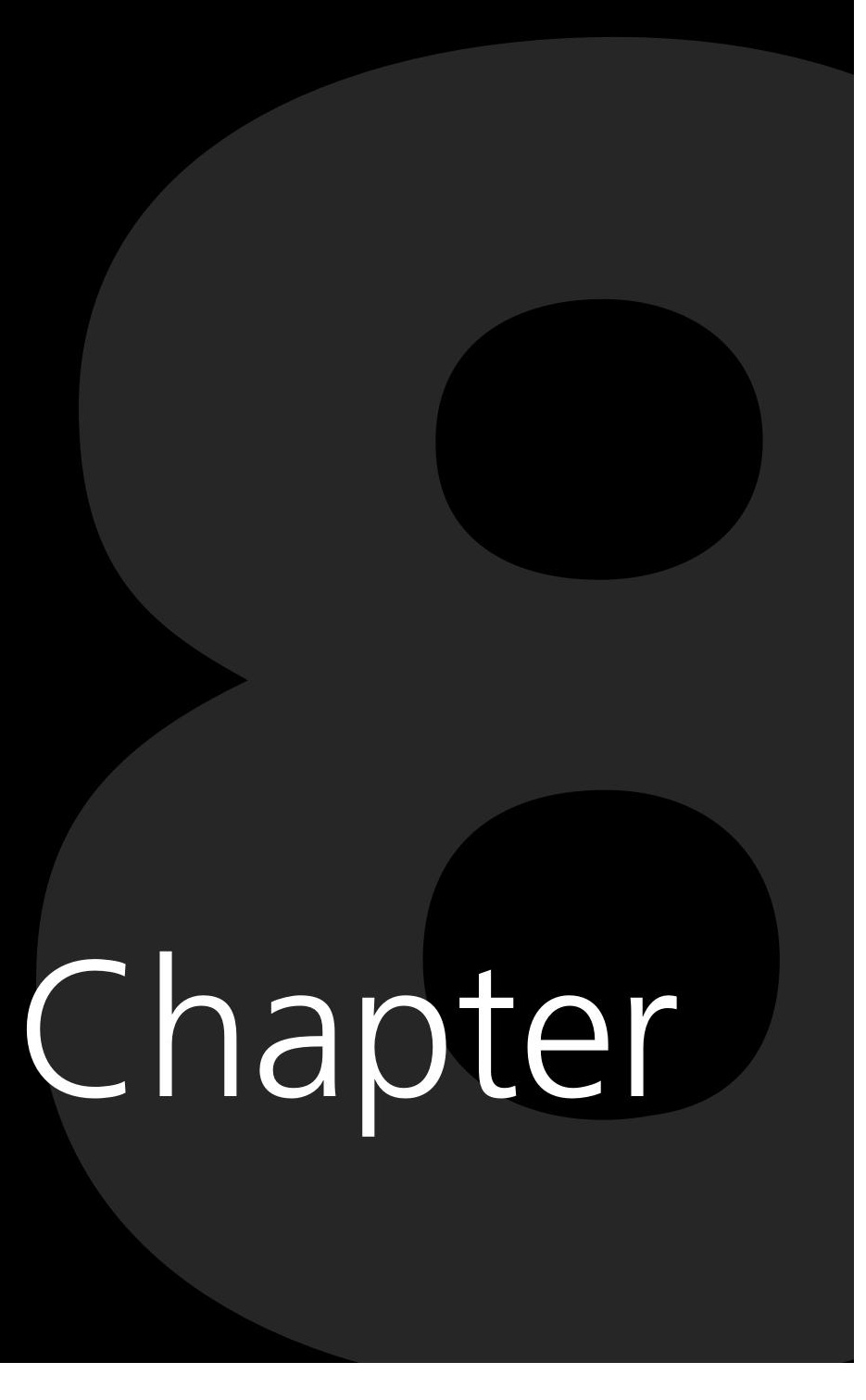





\title{
Neuroanatomical correlate of apathy in Alzheimer's disease
}

Submitted for publication

Pauline Aalten, Jeroen A. van Deursen, Paul A. Hofman, Frederik Barkof, Frans R. Verhey

\begin{abstract}
Apathy is among the most prevalent and severe neuropsychiatric symptoms in Alzheimer's disease (AD). The pathogenesis of apathy, and in particular of its sub-dimensions, has not yet been clarified. The aim of the present study was to investigate structural neuroimaging correlates of apathy while taking the following sub-dimensions into account: emotional blunting, lack of initiative and lack of interest.

Gray matter (GM) volumes were determined by using voxel-based morphometry. Apathy was assessed in the case of 37 AD patients with the help of the Neuropsychiatric Inventory, the Apathy Evaluation Scale (AES) and the Apathy Inventory. The results showed that one sub-dimension of apathy, i.e. emotional blunting was associated with decreased GM densities in the anterior cingulate cortex; in the left caudate nucleus; in the left superior temporal gyrus; in the right middle temporal gyrus; in the right insula; and in the left lingual gyrus. Furthermore, apathy as defined by the AES was related to decreased GM densities in the left superior frontal gyrus.

These data support the hypothesis that several brain areas, including the prefrontal sub-cortical areas, are involved in the pathogenesis of apathy and stress the role of neuronal circuits in mediating the different aspects of apathy and in particular the emotional sub-dimension. Further neuroimaging studies
\end{abstract}


are needed to confirm and validate the hypothesis that specific neuroanatomical correlates support the presence of the sub-dimensions of apathy and to determine its specificity across neuropsychiatric diseases.

\section{Introduction}

At present, it is widely acknowledged that apathy is an important neuropsychiatric syndrome. Apathy is among the most prevalent and severe neuropsychiatric symptoms (Aalten et al., 2005a; Steinberg et al., 2008; Steinberg et al., 2003); it has a negative impact on the quality of life of both patients and their caregivers; it is associated with caregiver distress; and it increases the likelihood of institutionalization (de Vugt et al., 2006; Shin et al., 2005; Tun et al., 2007). There is also evidence that apathy is a prominent feature of predementia stages and that it may be predictive for dementia (Robert et al., 2006a).

In recent years, there has been an enormous interest in new diagnostic criteria for identifying apathy in several neuropsychiatric diseases and consequently new specific tools for assessing apathy have been developed. Apathy is a distinct syndrome per se and not just a symptom of depression, as was recently confirmed in several studies including a recent study by the European Alzheimer's Disease Consortium (EADC) (Aalten et al., 2008; Aalten et al., 2007b). In addition, new diagnostic criteria for apathy stress the differentiation of apathy into several sub-dimensions, e.g. motivation, interest, initiation and emotional blunting (Robert et al., in press).

The pathogenesis of apathy, and in particular of its sub-dimensions, has not yet been clarified and treatment interventions are under development. Most consistently, apathy has been associated with the anterior cingulate cortex (ACC) in functional neuroimaging studies (Benoit et al., 2004; Benoit, 1999; Benoit et al., 2002; Craig, 1996; Lanctot et al., 2007; Marshall et al., 2007; Migneco et al., 2001), and structural neuroimaging studies (Apostolova et al., 2007; Bruen et al., 2008b; Rosen et al., 2005). Postmortem studies indicated that apathy is also associated with increased amounts of neurofibrillary tangles in the ACC (Marshall et al., 2006; Tekin et al., 2001). In addition to the ACC, the orbitofrontal cortex has been relatively consistently associated with apathy in several previous studies (Benoit et al., 2004; Benoit et al., 2002; Bruen et al., 2008b; Craig, 1996; Holthoff et al., 2005; Lanctot et al., 2007; Marshall et al., 2007).

Despite these consistencies most of these previous studies into the pathogenesis of apathy differed in their conclusion with regard to the laterality of 
the related hemispheres; they found inconclusive associations with several other brain areas and their conclusions were limited due to the fact that they assessed apathy by means of non-specific apathy assessments. In addition, only a few previous studies have taken the sub-dimensions of apathy into account (Benoit et al., 2004; David et al., 2008; Robert et al., 2006b). Therefore, the present study aims first of all to determine the structural correlates of the apathy syndrome by using several specific assessments of apathy and secondly to investigate the possible sub-dimensions of apathy.

\section{Methods}

\section{Patients}

Thirty-seven patients with a diagnosis of probable Alzheimer's Disease according to the DSM-IV and NINCDS-ADRDA criteria (McKhann et al., 1984) were included in this study. Standard blood work and neuroimaging (CT or MRI) were carried out and the diagnosis was supported by abnormal performance on neuropsychological tests. The main exclusion criteria were: a history of stroke; head trauma; and/or any other neurological or psychiatric disorders. Additional exclusion criteria were: severe cardiovascular disease; and/or a history of substance abuse; and/or other serious system diseases (e.g. malignancy, uncontrolled hypertension, neuropathy or seizure disorders). All AD patients were recruited at the Memory Clinic of the Maastricht University Medical Centre; they were not institutionalized and had a reliable informant. All the patients and a primary caregiver gave written informed consent prior to the study. The local Medical Ethics Committee of the Maastricht University Medical Centre approved the study.

\section{Apathy assessment}

Several tools were used in the present study to assess apathy:

\section{Neuropsychiatric Inventory (NPI) (Cummings, 1997; Cummings, 1994)}

The NPI is a semi-structured instrument for assessing the presence and severity of 12 neuropsychiatric symptoms in neuropsychiatric diseases. The severity and frequency of each symptom are scored on the basis of structured questions administered to the patient's caregiver. The continuous score for each symptom is obtained by multiplying severity (1-3) by frequency (1-4). In line with previous studies, a score greater than 3 was taken to indicate the presence of 'clinically relevant' symptoms (Aalten et al., 2005a; Lyketsos et al., 2002; Steinberg et al., 
2004). In the current study the Dutch validated version of the NPI was used (Kat et al., 2002a). Apathy was regarded both as a separate symptom and as a syndrome. The apathy syndrome was in accordance with the results of the EADC study and consisted of apathy and eating abnormalities (Aalten et al., 2007b). The syndrome was considered clinically significant when one or more of the accompanying symptoms scored greater than 3 on the NPI.

Apathy Evaluation Scale (AES-I) (Clarke et al., 2007; Marin et al., 1991)

The AES is an 18-item, informant-rated, 4-point Likert-type scale for the assessment of symptoms of apathy. Each question has four possible answers ranging from 1 to 4 (range $0-72$ ), with higher scores indicating higher levels of apathy. A cut-off score of $\geq \mathbf{4 0}$ has been used for determining the clinically significant presence of apathy.

Apathy Inventory (IA-caregiver) (Benoit et al., 2004; Robert et al., 2002)

This scale differentiates between three sub-dimensions of apathy, i.e. emotional blunting, lack of initiative and lack of interest. The IA is based on the format of the NPI: it rates frequency and severity for each dimension separately, then multiplies the outcomes to obtain a total dimension score (range 0-12), and IA total score (range 0-36).

\section{Neuropsychological assessment}

All the subjects underwent a neuropsychological test battery to measure their cognitive functioning. This included the Cambridge Examination for Mental Disorders of the Elderly part B (CAMCOG) (Derix, 1991; Roth et al., 1986), which incorporates the Mini-Mental State Examination (MMSE) (Folstein et al., 1975b). Sub-domains in the CAMCOG are: attention, orientation, language comprehension, expression, memory, praxis, abstract reasoning and perception. The neuropsychological evaluation further included: the Global Deterioration Scale (GDS) (Reisberg, 1982); Fluency (Lezak, 1995a); the Stroop Color Word Task (STROOP) (Houx et al., 1993); the ten-word learning task (10-WLT), which is derived from the auditory verbal learning task (Brand and Jolles, 1985); the Concept Shifting task (CST) (Vink and Jolles, 1985a); and the Letter-digit-symbol task (LDST) (Lezak, 1995a). A neuropsychological evaluation was available for 35 of the participants.

Structural MRI: scanning and analysis

A 3.0 Tesla Gyroscan NT MRI scanner $\left(\right.$ Phillips $^{\circledR}$ ) was used to obtain the following MRI scans: 3D-ISO T1W FFE, T2W-TSE, T2W-IR FLAIR, and IR-TSE scans. 
A coiled gradient was used, which provided high anatomical resolution and good grey/white matter contrast for subsequent segmentation and voxel-based morphometry (VBM). The T1-weighted 3D-ISO scan was used for VBM analysis and was acquired using the following parameters: voxel size $=1 \times 1 \times 1 \mathrm{~mm}$; $\mathrm{TR}=8.1 \mathrm{~ms} ; \mathrm{TE}=3.7 \mathrm{~ms} ; \mathrm{TFE}=230 \mathrm{~ms} ;$ flip angle $=8^{\circ} ;$ matrix $=224 \times 224$ pixels; FOV $=224 \times 224 \mathrm{~cm}^{2}$.

Image preprocessing was performed using Statistical Parametric Mapping (SPM 5) (Wellcome Trust Centre for Neuroimaging). The first step was a 12parameter affine registration with the MNI template as a target. We chose not to perform a non-linear registration procedure, because a previous study of $A D$ patients showed mainly expansions/contractions in the lateral ventricles and minimal changes in the gyri and sulci after this procedure (Karas et al., 2008b). The registered images were segmented into gray matter (GM), white matter (WM) and CSF probability maps, using the SPM 5 segmentation priors. Registration accuracy of the gray matter probability maps was further enhanced by a registration method that uses alignment and scaling to spread the registration bias among the whole group. The transformation matrix is averaged by projection to a manifold, a method described in more detail in Karas et al. and Woods et al. (Karas et al., 2004; Woods, 2003). The registered gray matter volumes were smoothed using a Gaussian kernel filter set at $12 \mathrm{~mm}$ (full with half maximum) to remove individual variations of gyral anatomy.

The smoothed GM images were entered in a multiple regression model to calculate their linear correlation with the several apathy assessments (e.g. IA three dimensions and total score; apathy symptom and syndrome of the NPI; AES total score). Age and MMSE were entered in this model as covariates for all variables. In addition, t-tests were also performed to determine GM correlates of patients with and without clinical presence of apathy, as defined by the cut-off score of the NPI and AES. The $x, y, z$ coordinates of the areas with a significant correlation between GM density and scores on the apathy assessments were identified using the Talairach Deamon Client tool (www.talairach). An image of the average GM volume of the current population was used to map the significant results.

\section{Statistical analysis}

Statistical analysis of the behavioral data was performed using the Statistical Package for Social Sciences (SPSS), version 16.0. Descriptive statistics were used to describe the characteristics of the patients. Pearson's correlation coefficients were calculated to determine the relationships between the several apathy assessments and the clinical and neuropsychological correlates. 
Functional and structural brain markers of Alzheimer's disease | Chapter 8

\begin{tabular}{lcccc}
\hline Brain area & $\begin{array}{c}\text { Left (L)/ } \\
\text { Right (R) }\end{array}$ & $\begin{array}{c}\text { Brodmann } \\
\text { area }\end{array}$ & $\begin{array}{c}\text { MNI } \\
\text { coordinate }\end{array}$ & $\begin{array}{c}\text { Z-score at } \\
\text { maximum }\end{array}$ \\
\hline IA 'emotional blunting' & & & & \\
\hline Anterior cingulate & $\mathrm{L}$ & 24 & $-626-6$ & 5.69 \\
gyrus & $\mathrm{R}$ & 24 & $525-5$ & 4.44 \\
\hline Caudate nucleus & $\mathrm{L}$ & & $-59-1$ & 3.80 \\
\hline Insula & $\mathrm{R}$ & 13 & $-44-5-5$ & 3.96 \\
\hline Superior temporal & $\mathrm{L}$ & 22 & $-5812-4$ & 4.25 \\
gyrus & $\mathrm{L}$ & 38 & $-5115-18$ & 4.01 \\
\hline Middle temporal & $\mathrm{R}$ & 21 & $2911-34$ & 3.59 \\
gyrus & & & $573-14$ & 3.76 \\
& & $472-24$ & 3.59 \\
\hline Lingual Gyrus & $\mathrm{L}$ & 18 & $-4-80-2$ & 4.59 \\
\hline AES: clinical presence & & & & \\
\hline Superior frontal & $\mathrm{L}$ & 10 & -28566 & 4.10 \\
gyrus & & & -24472 & 3.30 \\
\hline
\end{tabular}

Table 1. Volumetric correlates of apathy.

\section{Results}

\section{Characteristics of the patients}

Twenty-five men (67.6\%) and 12 women (32.4\%) were included in the study. The mean age was $72.6 \pm 9.1$ years. The average MMSE and GDS were $20.4 \pm$ 4.1 and $3.5 \pm 0.9$ respectively, generally indicating the inclusion of patients in mild to moderate stages of dementia. Thirty-three AD patients were taking psychotropics at the time of the study; this included 30 patients on cholinesterase inhibitors, 1 on memantine, 8 on antidepressants, 1 on benzodiazepines and 1 using an antipsychotic drug. The proportion of patients taking a psychotropic was not different in apathetic and non-apathetic patients as defined by the NPI and AES cut-off scores. The mean scores of apathy as defined by the different assessments were as follows: NPI apathy score $2.6 \pm 2.9$, NPI apathy syndrome score $4.1 \pm 4.3$, IA emotional blunting $0.97 \pm 2.4$, IA lack of initiative $3.2 \pm 3.7$, IA lack of interest $2.7 \pm 3.2$, and AES total score $41.8 \pm 8.6$.

\section{Clinical and neuropsychological correlates of apathy}

No relationship was found between any of the apathy definitions with the clinical variables gender, age, GDS, and MMSE. The sub-dimension 'emotional blunting' of the IA was significantly correlated with the sub-domain comprehension of 
the CAMCOG $(r=-0.48 p=0.004)$. The interference score of the STROOP was significantly correlated with both the NPI apathy symptom score $(r=0.39$ $p=0.05)$ and the sub-dimension 'lack of interest' of the IA $(r=0.50 p=0.009)$.

\section{Volumetric correlates of apathy}

As shown in table 1, the voxel-based multiple regressions showed significant relationships between decreased GM densities and the sub-dimension 'emotional blunting' of the IA and the clinical presence of apathy as defined by the AES. High scores of emotional blunting correlated significantly with decreased GM densities in the bilateral ACC (Brodmann area (BA) 24), the left caudate nucleus, the left superior temporal gyrus (BA 22,38), the right middle temporal gyrus (BA 21), right insula (BA 13), and the left lingual gyrus (BA 18). Patients with clinical significant apathy defined by the AES $(n=25)$ had decreased GM densities in the left superior frontal gyrus (BA 10).

\section{Discussion}

The aim of the present study was to determine the volumetric correlates of apathy in $A D$ and in particular to investigate the sub-dimensions of apathy. The most prominent finding of the present study was that emotional blunting is related to GM reduction in several frontal-subcortical brain areas, but that this was not the case with regard to the other sub-dimensions investigated, i.e. lack of initiative and lack of interest.

Specifically, AD patients with emotional blunting showed decreased GM densities in the bilateral ACC; in the left caudate nucleus, the left superior temporal gyrus; in the right middle temporal gyrus; in right insula; and in the left lingual gyrus. The significant clinical presence of apathy, as defined by the AES, was also related to the orbitofrontal gyrus (BA 10), a finding which is in agreement with several previous studies (Benoit et al., 2004; Benoit et al., 2002; Bruen et al., 2008b; Craig, 1996; Holthoff et al., 2005; Lanctot et al., 2007; Marshall et al., 2007).

Only a few neuroimaging studies have focused on the different subdimensions of apathy, hence data remains inconclusive. Robert and colleagues (Robert et al., 2006b) found that lack of initiative and interest were related to reduced perfusion of the right $A C C$, but concluded that emotional blunting was not related to the cingulate area. On the other hand, Benoit et al. (Benoit et al., 2004) found specific regions to be associated with the different dimensions i.e.: lack of initiative negatively correlated with reduced perfusion in the right 

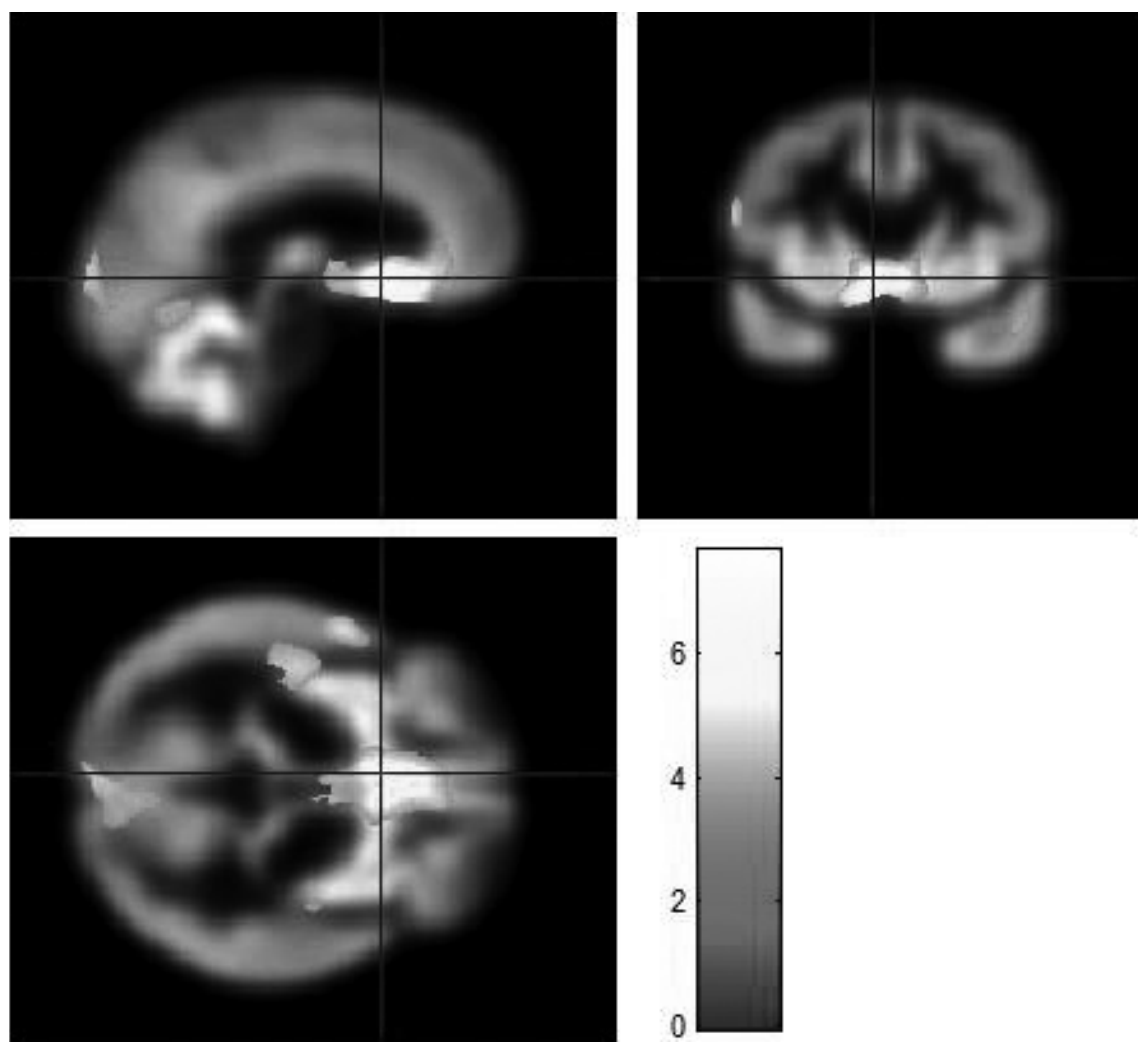

Figure 1. Brain areas showing a significant correlation to the sub-dimension concerned with emotional blunting as assessed by the IA: (A) Anterior cingulate cortex and (B) Superior temporal gyrus.

ACC; lack of interest with reduced perfusion in the right middle orbitofrontal gyrus; and emotional blunting with reduced perfusion in the left superior dorsolateral prefrontal cortex. David et al. (2008) focused on the relationship between apathy and striatal dopamine uptake, but only found that lack of initiative was related to dopaminergic neuronal loss.

The inconclusive data regarding specific brain correlates of specific sub-dimensions of apathy were all based on the use of the IA. Since the present study is the first structural imaging study that takes the sub-dimensions of apathy into account, this might explain the differences with the other studies. Although at present there is diagnostic consensus about the presence of different sub-dimensions of apathy, these sub-dimensions need to be validated by future neuropathologic studies.

Nevertheless, the finding of this study that the frontal-subcortical brain areas are related to the sub-dimension 'emotional blunting' is in line with the 

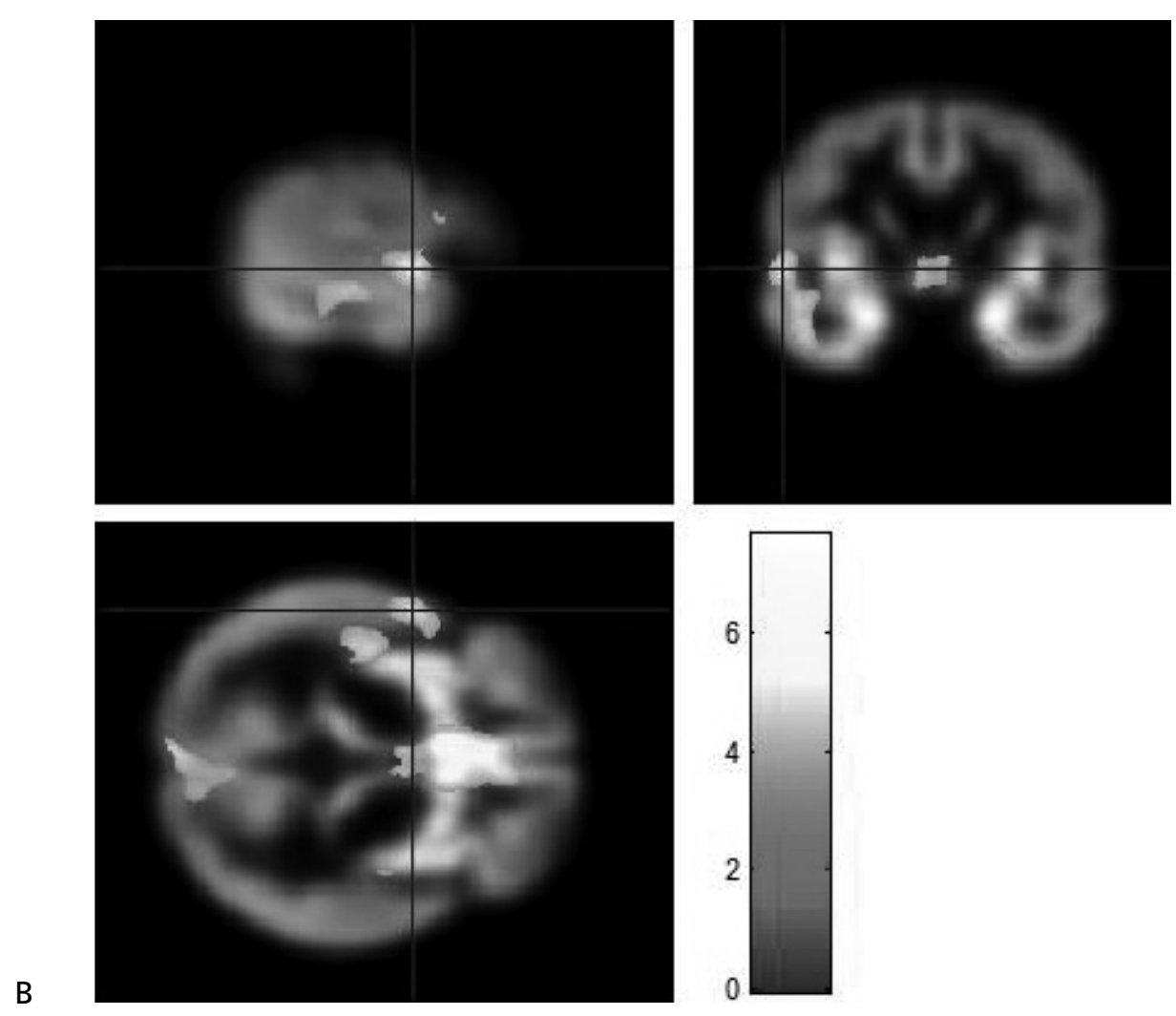

structures reported by previous neuroanatomical studies of apathy, as well as with the functional neuroanatomy of emotion as reviewed by Phan et al. (Phan et al., 2002). The latter study concluded that the medial prefrontal cortex is involved in emotion, and that the ACC and the insula in particular are related to the integration of cognitive and affective processing. The insula is preferentially involved in the evaluative and expressive aspects of internally generated emotions. The basal ganglia, including the caudate nucleus, are related to the positive aspects of emotion related to e.g. reward processing and have a rich innervation of mesolimbic dopamineric neurons. A study by Bruen et al. (Bruen et al., 2008b) also using VBM showed that the left caudate nucleus was related to apathy in $A D$ patients, as well as being related to reward mechanisms.

Little is known about neurochemical mechanisms of apathy in $A D$, but recently investigators have suggested that dopaminergic drugs such as methylphenidate, amantadine and bupropion, may be useful for reducing apathy in AD 
(Landes et al., 2001). Although the dopaminergic system is relatively preserved in $A D$, dopaminergic and related neurotransmitter changes may contribute to apathy (Boyle and Malloy, 2004). From studies of Parkinson's disease it is known that degeneration of dopaminergic cells is implied in the pathophysiology of several non-motor symptoms related to initiation, motivation and reward, such as depression and apathy (Lawrence et al., 2003).

Levy and colleagues (Levy and Czernecki, 2006; Levy and Dubois, 2006) also stressed the relation of apathy to prefrontal and basal ganglia lesions, as a consequence of the disruption of the prefrontal-basal ganglia axis. Emotional blunting is one of the main features of orbital-medial prefrontal cortex dysfunction and provides the key to understanding this sub-dimension of apathy. The circuit is essential to provide the contextual value of reward and consequently to integrate the rewarding value of a stimulus into behavior. Dysfunction of this circuit might lead to an insensitivity to reward, which may in turn lead to a decreased number of voluntary actions related to e.g. emotion and apathy. Subtypes of apathy may depend on which brain region is affected. Studies focusing on both emotion and apathy suggest that neural networks, including those of both the cortical and subcortical areas, are involved in the pathogenesis of apathy and not specific regions of the brain. Whether this is in particular related to the emotional sub-dimension of apathy requires further study.

The present study showed that reduced GM density in the temporal cortex was associated with apathy. The association of temporolimbic structures and apathy in AD has already been suggested by Marin (Marin, 1991), who mentioned that disruption of these structures may lead to loss of integration of emotional and sensory information necessary for appropriate goal-directed behavior and motivation. In addition, in line with the present study, Lanctot et al. (Lanctot et al., 2007) also reported a relationship between apathy and the left superior temporal gyrus and right middle temporal gyrus. The temporal gyri together with the orbitofrontal areas, the ACC and caudate nucleus are crucial parts of the brain's reward system. Migneco et al. (Migneco et al., 2001) also found that the lingual gyrus is related to apathy in AD patients.

There has been no clear evidence for laterality in either the ACC or orbitofrontal areas related to apathy. Previous studies reported as follows: bilateral (Apostolova et al., 2007; Bozzola, 1992; Bruen et al., 2008b; Marshall et al., 2007; Migneco et al., 2001; Ott et al., 1996; Rosen et al., 2005); predominantly left (Benoit et al., 2002; Lanctot et al., 2007); and predominantly right hemispheres (Benoit et al., 2004; Robert et al., 2006b). The present study supports the finding that apathy is more closely associated with bilateral hemispheres, but depending on which aspect of apathy is concerned one of the 
hemispheres is more specifically involved. Again, this supports the hypothesis that neural circuits are involved in the pathogenesis of apathy, and not only one particular brain area.

In line with previous studies (Bozzola, 1992; Ott et al., 1996; Rosen et al., 2005), apathy was independent of several clinical characteristics of the patients. However, emotional blunting was related to language comprehension, which may support the relation between apathy and the caudate nucleus and temporal-limbic structures. In addition the relation of apathy with executive functioning, as determined by the STROOP, supports the role of prefrontal structures in the pathogenesis of apathy. The associations between cognitive disturbances and apathy support our GM atrophy findings.

The present study was limited by the relatively small sample of $A D$ patients with apathy that participated. The generalization of the results to other neuropsychiatric diseases and research including larger samples deserve further attention. For example, Rosen et al. (Rosen et al., 2005) only found neuroanatomical correlates of apathy in a frontal temporal dementia and semantic dementia group, but not in AD patients.

However, the main strengths of the present study were first of all the use of VBM, which made it possible to simultaneously look at several of the brain areas involved, and secondly the use of several specific apathy scales, which enabled a focus on the different sub-dimensions of apathy. Another strength was the use of 3 Tesla MRI scans, which increased segmentation accuracy. To the best of our knowledge the present study is the first to determine structural brain correlates for the sub-dimensions of apathy. Future neuroimaging studies are needed to validate the presence of the sub-dimensions of apathy as defined by recent diagnostic criteria by determining their specific neuroanatomical correlates.

The most important conclusion supported by this study is that networks, including those in the prefrontal-subcortical areas, are involved in the pathogenesis of apathy and not specific regions of the brain. Yet, there is no clear view on how these different networks are integrated and on what level they contribute to the different sub-dimensions of apathy. These results have to be confirmed by future studies that investigate the sub-dimensions of apathy in larger samples and determine its specificity with regard to other neuropsychiatric diseases. Eventually, the results might lead to better treatment options for this stressful neuropsychiatric symptom. 
Functional and structural brain markers of Alzheimer's disease | Chapter 8

\section{Funding}

This work was supported by the Dutch Hersenstichting [grant number 12F04.39].

\section{Acknowledgement}

We would like to thank Marc Geerlings and Laura Schellekens for their help in data acquisition and analyses. 


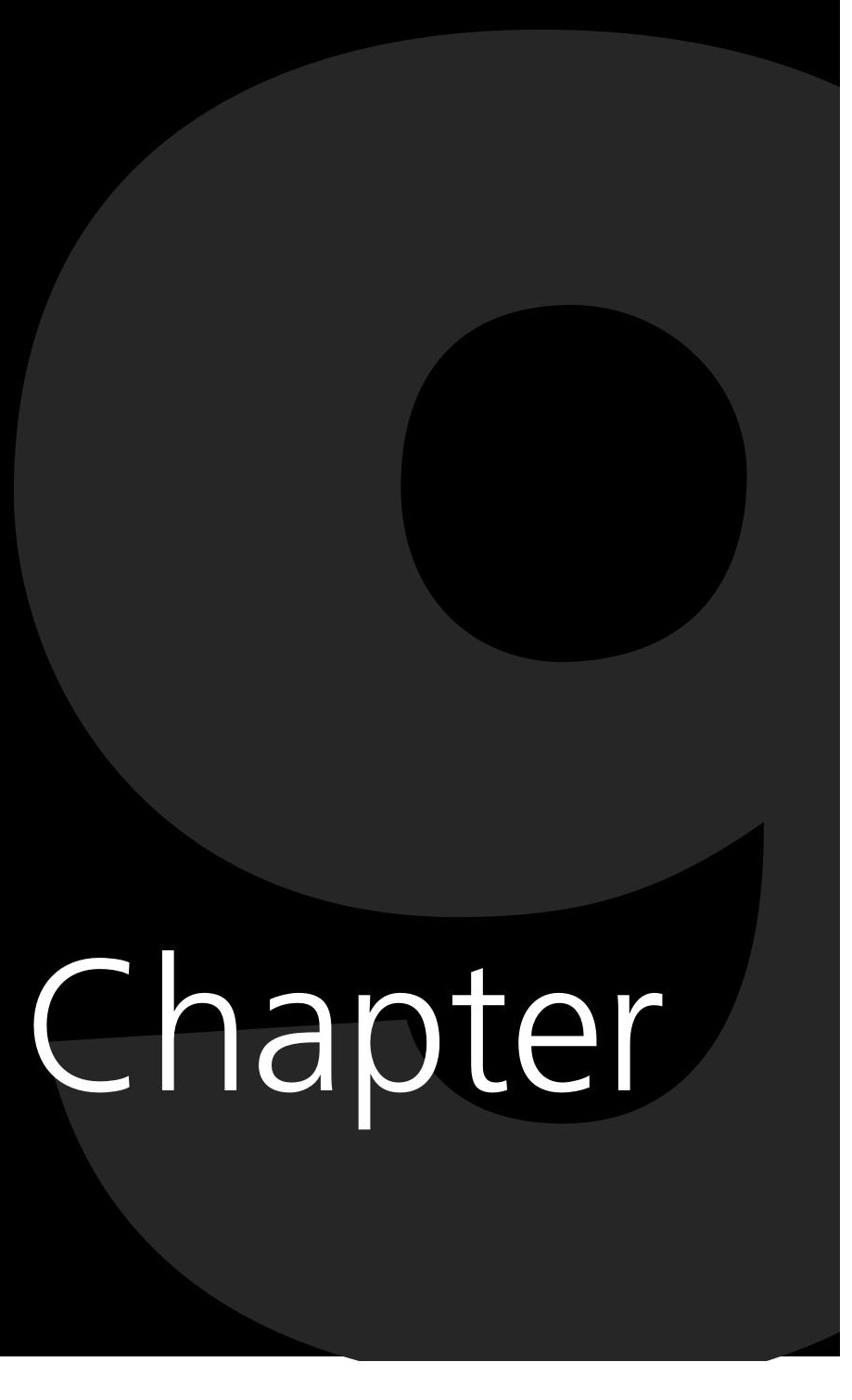





\section{General Discussion}

\subsection{Introduction and summary of main findings}

Alzheimer's Disease (AD) is the most common form of dementia with a rapidly increasing prevalence. $A D$ is characterized by a progressive decline of cognitive abilities and the presence of neuropsychiatric disorders. The clinical construct of $A D$ is a complex one as it is mainly based on the phenomenological features of the disease. These phenomenological features mainly involve changes in behavior: cognitive impairment; neuropsychiatric disorders and impairment of daily life activities. The substrates for these behavioral changes that hallmark $A D$, can be studied in terms of looking at changes in the brains' function and structure. At present a challenging gap remains in linking the phenomenology of onset and progression of AD with specific changes in neural substrates. The development of biological markers can play a crucial role in this process. A biological marker is defined as a characteristic that is objectively measured and evaluated as an indicator of normal biologic processes, pathogenic processes or the response to a therapeutic intervention. New developments in the field of biological markers could provide new insights in aspects of the pathogenesis and progression of $A D$ as well as in the development of new treatments strategies. Furthermore, these biomarkers could contribute to increased diagnostic accuracy and decrease the necessary trial size in treatment intervention studies. A wide variety of candidate biomarkers of $A D$ are already available. These were reviewed in chapter 2 . The present thesis puts forward a multidimensional approach in which changes in brain function and structure are studied in relation to cognitive and behavioral observations. 
The present thesis had two main aims. The first was to evaluate the possible role of EEG measures in the diagnosis and follow-up of Alzheimer's disease (AD) and to relate these findings to cognition. The second aim was to examine the structural changes in the brain that are associated with the presence of neuropsychiatric disorders in $A D$.

The first part of the thesis (chapter 2 through 6 ) showed that most EEG paradigms could discriminate $A D$ patients from $\mathrm{MCl}$ patients and healthy control subjects. The results of this thesis also showed that most EEG paradigms could reliably be reproduced in this elderly, cognitively impaired population. In addition to the EEG paradigms, response speed as measured by a choice reaction time task (CRT) also showed to be very sensitive, specific and reliable in discriminating between $\mathrm{AD}, \mathrm{MCl}$ and healthy controls.

In the second part of this thesis, the neuroanatomical correlates of neuropsychiatric disorders in AD were examined. Chapter 7 showed that the syndromes hyperactivity and psychosis were associated with atrophy in specific regions of the frontal cortex, temporal cortex and basal ganglia. Chapter 8 further shows that apathy and emotional blunting were associated with atrophy of specific areas in the frontal cortex, temporal cortex and basal ganglia.

In this final chapter, these findings will be briefly summarized, discussed and completed with recommendations for future research.

\subsection{EEG as a biomarker for AD}

\subsubsection{Gamma band oscillations}

One of the hallmarks of $A D$ is that patients lose the ability to process new information in an efficient manner. Recent evidence shows that high frequency neural oscillations are an important mechanism in the integration and binding of information during cognitive processes (Basar et al., 2001; Kaiser and Lutzenberger, 2003; Kaiser and Lutzenberger, 2005; Singer and Gray, 1995). These neural oscillations can be detected using EEG measures (ie gamma band power across 30-100 Hz). High frequency gamma band oscillation can be induced in different ways; through the presentation of specific stimuli which evoke a short response of gamma band activity; through continuous sensory stimulation, the so called induced gamma band response; and through auditory stimulation at $40-\mathrm{Hz}$ frequency, which evokes a steady state response (SSR) (Tallon-Baudry and Bertrand, 1999).

In this thesis the focus was on the induced gamma band response and the $40-\mathrm{Hz}$ SSR. It was shown that in both paradigms gamma band power was in- 
creased in $\mathrm{AD}$ compared to $\mathrm{MCl}$ and healthy controls, matched for age and gender. On the basis of previous studies, which indicated that healthy controls $A D$ patients relative to control subjects showed a decrease of gamma band synchronisation, we expected to find decreased gamma band power (Stam et al., 2002). Synchronisation or coherence of the EEG basically reflects synchronous neural activity in different groups of neurons in close proximity or at a longer distance. This synchronous activity in different brain areas is assumed to be involved in the integration of information processes from different brain areas. Previous work showed that synchronisation in $A D$ is decreased mainly in the longdistance connections (Babiloni et al., 2004; Stam et al., 2006). When the findings of the present thesis are compared to previous synchronisation studies it can be argued that in $A D$ mainly the long distance projections are affected but that the small local networks remain intact. Hence, the observation of increased induced gamma band response as well as increased $40-\mathrm{Hz}$ SSR can be considered as a compensation mechanism for ineffective integration of neural processes. One could hypothesize that there is an inverse relation between gamma band synchronisation and power during the progression of $A D$. Until now most EEG and MEG studies involved mild to moderate AD patients. With regard to future research it would be interesting to examine if gamma band power continuously increases simultaneous with the progression of $A D$. It would be expected that at some point in the progression also the small neural networks become insufficient, resulting in a decrease of local gamma band power. Furthermore, it would be interesting to investigate how synchronisation is associated with EEG power in general and to address the question if this association is abnormal in AD.

\subsubsection{The ERP's and response speed tasks}

Decreased response speed is a well-known phenomenon seen in normal aging as well as in AD. Several studies have shown that response speed changes are very sensitive in discriminating $A D$ patients from $\mathrm{MCl}$ and healthy controls (Gordon and Carson, 1990; Levinoff et al., 2005). Besides employing crude reaction time (RT) measures as a function of the amount of information processed per units of time, event-related potentials may provide more specific information about the degree to which the assumed specific stages of information processing between stimulus and response are specifically slowed down or impaired. By comparing markers across early, intermediate and late aspects of information processing, one may draw conclusions whether the observed slowing of information processing is specifically due to any of the early perceptual stages of stimulus identification, the middle effortful decision-making stage, also indexed by P300 amplitude and latency in the P300 oddball paradigm, or the 
late stages concerning motor preparation and response execution, also indexed by CNV amplitude in a cued response paradigm (Sanders, 1998). In the present study Simple RT and Choice RT yielded Reaction Times only differing in cognitive load of 1 bit of information (no response choice versus 1 binary response choice). The fact that AD patients are impaired on both SRT and CRT but needed relatively much more time when a decision is required indicates that slowing is more outspoken with increased cognitive load. The present study showed significant difference between the groups in response speed and the P300 components. There was a reduced P300 amplitude and increased latency in $\mathrm{AD}$ compared to $\mathrm{MCl}$ and healthy controls. Both amplitude and latency were correlated significantly with response speed, which suggests that $A D$ patients are less efficient in distinguishing a target from a non-target. The present thesis showed that readiness to respond was not impaired in $A D$ as there was no difference in CNV between the groups. The findings in the present thesis suggest that response speed tasks that require conscious attention or some form of decision-making are probably most sensitive in discriminating patients from other groups, such as $\mathrm{MCl}$ and controls. The behavioral and neurophysiological findings, regarding this aspect are in agreement as the basic tasks SRT and CNV show less differences between the groups than the more complex task; the CRT and oddball task. Based on these findings we can conclude that response speed tasks become more sensitive to detect changes in $A D$, when the tasks are more complex and require more cognitive resources.

\subsubsection{Implications of findings}

The findings presented in this thesis show that EEG can provide measures, which can be useful as a sensitive and reliable method to discriminate $A D$ from $\mathrm{MCl}$ and healthy controls. Nevertheless, based on our findings we have to conclude that these EEG measures will have little additional value to the current diagnosis of AD. The accuracy of the clinical diagnosis and cognitive tests exceeds that of the EEG paradigms evaluated here. However, the response speed tasks and especially the CRT showed a high diagnostic accuracy in discriminating between the patient groups. That the CRT will have an additional value as a diagnostic instrument is not very plausible however, as decreased response speed is also present in other forms of dementia. Besides the question of the diagnostic accuracy it is also important to study whether EEG might have an additional value as an instrument to monitor disease progression and the effects of treatment interventions. Although this question in itself was not studied in present thesis, two important prerequisites of a biomarker for longitudinal follow-up were evaluated; sensitivity to detect the disease and test-retest reliability. Both 
the sensitivity and test retest reliability of most of the EEG parameters were satisfactory. These findings suggest that EEG provides valid measures and hence additional sources of information with regard to the follow-up and treatment interventions in AD. An advantage of EEG measures over clinical rating scales is that EEG provides measures of disease-related changes in a more objective manner, as clinical rating scales that are used in the longitudinal follow-up and evaluation of treatments often rely in part on subjective judgments by the treating physician or the patient's informant. These biases would not affect changes provided by EEG measures.

During the follow-up of the more cognitively impaired AD patients it is often difficult to monitor the status with cognitive tests, since these patients often have difficulty in understanding the task instructions. In the present thesis several passive tasks were studied (i.e. resting state, music listening, story listening and visual stimulation). An advantage of these tasks is that they require limited task instructions and hence can also be performed properly, by severely impaired patients. During the follow-up and also when monitoring treatment effects in more advanced stages of $A D$, these passive EEG tasks might provide valuable information.

\subsubsection{Methodological considerations}

In the methods that are used in EEG research of AD there is large variability. Several of these methodological aspects can have an effect on the outcome of the study. In clinical studies the severity of the disease of the population being studied is one of these aspects. In the current thesis the AD population was mild to moderately impaired. The participants were capable of understanding and performing the task instructions.

An important aspect in EEG studies of $A D$ is the use of psychoactive medication. Several studies have shown that cholinesterase inhibiting drugs and other psychoactive medications have a direct effect on the EEG frequency and ERP's (Kogan et al., 2001; Rodriguez et al., 2002; Werber et al., 2003). This confounding aspect is often underestimated as most EEG studies include AD patients who are using cholinesterase inhibiting drugs and other psychoactive substances at the time of examination. As the present thesis aimed to evaluate EEG as a candidate biomarker, we considered it of major importance to rule out this potential bias. Although this is a methodologically strong aspect of this study, its consequence was that the inclusion rate was quite low, which resulted in a lower $\mathrm{N}$ than we initially aimed for.

In two chapters of this thesis we focused on high frequency gamma band oscillations (30-100 Hz). A disadvantage of studying this frequency band is that 
the EEG signal can be contaminated with electromyographic (EMG) signals (Whitham et al., 2007). In the present studies we reduced this potential bias by filtering the data and visual inspection. All EEG epochs that contained EMG were omitted from analysis. This removal of EMG resulted in a general decrease of gamma band power, while the main group differences remained the same. Despite our efforts to reduce the effect of EMG on our results, it cannot be excluded that there was no EMG activity left in our data. Our findings together with those from Whitham et al. (2007) underline that for future studies it is important to address this aspect. Ideally, future development will lead to procedures that remove EMG contaminations in an automated manner.

Another methodologically important issue that was addressed in the present thesis is the assessment of the neurophysiological paradigms and the cognitive measures in a test-retest design allowing evaluation of the test-retest reliability. The good test-retest reliability of a new test is an important aspect that is often underestimated in neuroimaging studies. Most of the EEG paradigms proved to be reliable in terms of test-retest reliability. The high reproducibility of the EEG paradigms ensures that these parameters can reliably be used to monitor changes in studies with a longitudinal design.

Our conclusions regarding the specificity of the variables studied here were limited to three groups only differing in the severity of cognitive impairment. Comparison to other neurodegenerative disorders would give more insight in the specificity of the measures.

\subsection{VBM as a biomarker for neuropsychiatric disorders in AD}

\subsubsection{Implications of findings}

Neuropsychiatric disorders are a common feature of AD. In $95 \%$ of the patients, neuropsychiatric disorders are present at some point in time (Aalten et al., 2005a; Steinberg et al., 2006). The presence of neuropsychiatric disorders in $A D$ have a negative impact on the quality of life (Shin et al., 2005) of the patients and are a major burden for the caregivers (de Vugt et al., 2006). At present the pathogenesis of neuropsychiatric disorders in $A D$ is not clear. Furthermore there is a large variability in the type of neuropsychiatric disorders that are present in AD. Of all neuropsychiatric disorders apathy is the most common and severe (Aalten et al., 2005a; Steinberg et al., 2008). Most studies on neuropsychiatric disorders in AD focused on the presence of individual neuropsychiatric symptoms. Recent evidence suggests that neuropsychiatric symptoms in $A D$ rarely occur in isolation and are often present in clusters of symptoms: syndromes (Aalten et al., 2007b; Frisoni 
et al., 1999; Lyketsos et al., 2001). The association between individual symptoms suggests that there is a common pathogenesis.

Structural neuroimaging studies can provide more insight in the pathogenesis of neuropsychiatric syndromes in AD. In the present thesis we focused on the association between local gray matter atrophy and the presence of neuropsychiatric syndromes and the sub-domains of apathy. Our findings suggest that there is a relation between atrophy of the frontal cortex, temporal cortex and basal forebrain with the presence of the neuropsychiatric syndromes hyperactivity, psychosis and the sub-domains of apathy. As this was the first study to show an association between local gray matter atrophy and the presence of neuropsychiatric syndromes and the sub-domains of atrophy, these findings should be confirmed by future studies. Future studies should also focus on the question how biological aspects, such as gray matter atrophy, interrelate with psychological and social factors in the pathogenesis of neuropsychiatric disorders. Eventually more insight in the pathogenetic processed that are involved in the development of neuropsychiatric disorders during the course of disease will provide new leads for better therapeutic strategies of these disorders. Better treatment strategies should alleviate the burden for the patients and their caregivers.

\subsubsection{Methodological considerations}

In the present thesis voxel-based morphometry (VBM) was used as an instrument to analyze the association between neuropsychiatric disorders and gray matter volume.

The VBM method has been a matter of debate amongst neuroimaging specialists for several reasons. One of the main objections against VBM is that during the spatial normalization procedure, in which the subjects brain is warped on a standard brain, volume difference induce mis-registration biases (Bookstein, 2001). These mis-registration biases are even greater when degenerated brains are studied, as these patients' brains deviate from the standard template brain. In the studies of the present thesis a non-standard approach was used in order to minimized these potential biases. This approach provided an alternative for optimized VBM, in which an average scan of the population is used (Good et al., 2001). During the registration procedure a linear 12-parameter affine registration was applied to a standard brain, which reduced the variance in expansion and contractions in the ventricles, gyri and sulci. After segmentation all individual transformation matrices were projected onto random scans of the group using a semi-Riemannian manifold and averaged, thus creating a common transformation matrix for all segmented scans (Karas et al., 2004; Woods, 2003). 
When this custom-made transformation matrix was applied to all segmented images, they converged towards the common target. This method spreads the registration bias over the whole group and converges all individual scans in proximity to all other scans in the group.

Another objection against VBM is that the sulci and gyri vary between different individuals, and that VBM does not account for these individual differences. In general this bias is reduced with the help of the smoothing procedure. In the smoothing procedure, which is usually applied at the end of the preprocessing procedure, a Gaussian kernel is used. The result is that the gray matter density of a voxel attains the average concentration of the surrounding tissue. The smoothing procedure also partly compensates for the registration bias. These methodological aspects are important for the accuracy of VBM analysis when degenerated brains are being studied.

Despite these criticisms on VBM, the method has some major advantages over other neuroimaging techniques. First of all, in contrast to the region of interest (ROI) approach in structural neuroimaging, VBM allows one to study the whole brain at once. This is a major advantage especially in explorative studies in which there are no a-priori assumptions of specific brain areas of interest. Nevertheless when there is a very specific a-priori hypothesis the ROI approach is probably more reliable. Secondly, VBM has an advantage over functional imaging methods, such as SPECT and PET, due to its higher spatial resolution especially when the MRI images are acquired on a 3-T scanner. And thirdly, VBM has an advantage of a more practical nature because the analysis time with VBM is much shorter than that of an ROI analysis, which is usually performed manually.

In addition to these image analysis aspects, there are some important issues concerning the use of ratings scales to assess neuropsychiatric disorders. In this thesis the NPI (Cummings, 1997), was used to assess the presence of neuropsychiatric symptoms which were clustered into syndromes. Furthermore, the AES (Marin et al., 1991) and Al (Robert et al., 2002) were used to assess apathy. All these scales rely on the information provided by an informant, and in general this will be the primary caregiver who is usually a close relative or spouse. Although it is generally acknowledged that the information provided by an informant is more reliable than the information provided by the patients themselves, this information may be biased by various aspects, such as informant mood, stress experienced by the informant, problems in the relationship and coping style. 


\subsection{Future perspectives}

Based on the findings in the present thesis, some recommendations for future research can be formulated. The findings of the present thesis suggest that neither EEG nor VBM will have a major role as a diagnostic instrument in clinical settings. Nevertheless, both methods provide valuable information on the functional and structural changes of the brain associated with AD pathology.

Of the EEG paradigms that were studied in the present thesis, the gamma band tasks were the most novel. The changes in the gamma band showed a relation with the cognitive performance of the subjects. Despite this relation it is still unclear which mechanisms are involved in the differences between the groups that were found in our studies. Earlier in this discussion we argued that the relation between synchronisation of the gamma band and gamma band power should deserve more attention in future research. Another important focus of future research should be to provide more insight in the neuroanatomical and neurochemical substrate of the human gamma band. As the neuro-anatomy cannot be manipulated, the first studies to address this question should manipulate neurotransmitter systems to study their role in the regulation of the gamma band. Several in-vitro (Hasselmo and Fehlau, 2001; Traub et al., 1996; Whittington et al., 1995) and in-vivo studies (Ahveninen et al., 1999; Jaaskelainen et al., 1999) have suggested that acetylcholine, glutamate and gamma-amminobutyric acid (GABA) are important modulators of the gamma band, but their exact role is still unclear. In order to gain more insight in the neuro-anatomical underpinnings of the EEG changes in $A D$, a link should be made between neural structures and their neurophysiology. It would be interesting to study how atrophy of a specific brain area alters the neurophysiological function of this area. Voxel-based morphometry could play a role in providing information on the link between the volume of a structure and the changes in the EEG. Other techniques, such as magneto-encephalography (MEG), which has a better spatial resolution than EEG, could also contribute in addressing such research questions.

With regard to the volumetric studies in this thesis, there was a clear relation between atrophy of the frontal cortex, temporal cortex and the basal forebrain with the presence of neuropsychiatric disorders. In chapters 7 and 8 it was pointed out that a similar relation has been shown with the load of neuropathological changes in post-mortem studies. It is most likely that not local atrophy, but a disruption of neural networks ultimately results in the presence of neuropsychiatric disorders. At present it is not clear how these neural networks are composed, and in what way they contribute to the onset 
of neuropsychiatric disorders. Future research should try to gain more insight in these neural networks by studying the interrelationship between gray matter changes, white matter changes and also the load of neurofibrillary tangles and amyloïd plaques by using PET ligand imaging.

Another point of interest for future research would be to predict the effects of treatment prior to treatment onset or after a very short period of time. The drugs that are currently used in AD do not work for every patient and can cause considerable side effects. Furthermore, these drugs are very expensive. From the patient's point of view and also from a health care cost perspective it would be favorable if it was possible to predict the individual response to a treatment. Structural neuroimaging studies of the medial temporal lobe volume have attempted to predict the effect of rivastigmine without success (Visser et al., 2005). On the other hand an EEG study which focused on the low EEG frequencies, retrospectively showed a significant difference in theta band power between responders to rivastigmine treatment and non-responders (Adler et al., 2004). Whether one of the EEG paradigms studied in the present thesis will be able to make a reliable prediction is unclear, but would be an interesting subject for future research. It is likely that EEG will be more sensitive within the time frame in which drugs that affect neurotransmitter systems, while VBM will be likely to be more sensitive within the much longer time frame for future disease modifying agents to exert measurable action.

The present thesis showed that biomarkers could provide new and important information in AD research. The multidimensional approach provided new insights in the relation between clinical phenomena and changes in the neural substrate. The enormous variety of biomarkers has led to little fragmented pieces of knowledge. Future research should focus on binding these pieces into a whole and to bridge the gap between clinical phenomena and changes in the neural substrate. 
Summary 

Alzheimer's Disease (AD) is the most common form of dementia and is characterized by progressive cognitive impairment and neuropsychiatric problems. At present there are no causal treatments that affect the pathological processes in AD. In search for a causal treatment there is a need for biological markers in order to gain more insight in the cognitive, neuropathologic, morphologic and neurophysiological changes in AD.

In the first part of the thesis the psychometric properties of measures obtained mainly from the electroencephalogram (EEG) were evaluated as candidate biomarkers to assist diagnosis and potentially as biomarkers to monitor disease progression, either as such or after interventions, of mild cognitive impairment (MCl) and AD.

The second part of the thesis examined the neural correlate of behavioral disorders in $A D$ using voxel-based morphometry (VBM).

For all the studies in this thesis, a total of $44 \mathrm{AD}$ patients, $20 \mathrm{MCl}$ patients and 20 healthy controls subjects were included. Eight AD patients participated in both the EEG and VBM studies.

In chapter 2 the available literature focusing on biological markers for the diagnosis and follow-up of $A D$ is reviewed. The aim was to compare EEG with other biological markers in order to determine the value of EEG measures as candidate biomarkers in AD. The value of EEG measures was compared to cognitive markers, biochemical markers and markers derived from anatomical- and functional neuroimaging data. Moreover, the reliability, sensitivity and specificity of these markers was assessed. The conclusion of this review was that EEG measures can yield valuable candidate marker for the follow-up and evaluation of therapeutic interventions in AD. The review further discussed that with regard to new EEG paradigms there is little knowledge on their sensitivity, specificity and reliability.

In chapters 3 through 6 inclusive, experimental data on a variety of electrophysiological measures, cognitive performance measures and behavioural measures are compared between samples of patients with $A D, M C l$ and Healthy Control subjects. The groups consisted of fifteen patients diagnosed as probable AD (11 male, 4 female) with a mean age (SD) of 75.2 (6.9) and a mean (SD) MMSE score of 20.8 (2.7, range 17-26), twenty MCI patients (12 male, 8 female) with a mean (SD) age of 70.6 (7.2) and a mean (SD) MMSE of 26.3 (1.6, range 23-29) and twenty healthy control subjects (12 male, 8 female) with a mean (SD) age of 69.5 (6.1) and a mean (SD) MMSE of $29.3(0.8$, range 28-30) completed the study. All participants were tested on all paradigms described in chapters 3 through 6 , in two assessment sessions. To evaluate test-retest reliability (TRR), subjects underwent a similar second assessment one 
week after the first. The NART and the MMSE were assessed only in the first session.

In chapter 3, high frequency $(30-70 \mathrm{~Hz})$ gamma band oscillations in the human electro-encephalogram (EEG) were compared between AD patients, subjects fulfilling the $\mathrm{MCl}$ criteria and healthy control subjects. Gamma band oscillations are thought to reflect perceptual binding, an improtant step in the processing of sensory information. Gamma band power (GBP) was measured in four conditions; resting state, music listening, story listening and visual stimulation.

The overall TRR was high. Elevated GBP was observed in AD when compared to $\mathrm{MCl}$ and control subjects in all conditions. The results suggest that elevated $\mathrm{GBP}$ is a reproducible and sensitive measure for cognitive dysfunction in $A D$ in comparison with $\mathrm{MCl}$ and controls.

In chapter 4, the $40-\mathrm{Hz}$ steady state response (SSR), which reflects early sensory processing and can be measured with electroencephalography (EEG), was assessed. Auditory click trains at a frequency of $40-\mathrm{Hz}$ evoked the $40-\mathrm{Hz}$ SSR. The results showed a high TRR and a significant increase of $40-\mathrm{Hz}$ SSR power in the $A D$ group compared to $\mathrm{MCl}$ and controls.

The findings suggest that $40-\mathrm{Hz}$ SSR might be an interesting candidate marker of disease progression.

In chapter 5, neurophysiological markers of decreased speed of information processing in $A D$ and $\mathrm{MCl}$ were studied. Recent studies suggested that response speed (RS) measures can be more sensitive indicators of change in $A D$ clinical trials when comparing to the standard instrument, ADAS-Cog. Insight into the potential neurophysiological underpinnings of slowed RS can be provided by measuring the associated event-related potentials (ERP). Response speed was measured during a simple reaction time (SRT) task and a choice reaction time task (CRT). An oddball paradigm and a contingent negative variation (CNV) paradigm were used to elicit ERP's. The SRT and CRT discriminated the patient groups significantly. The P300 amplitude and latency also discriminated the groups and showed a significant correlation with response speed. The CNV amplitude did not reveal a significant difference between groups and also showed a low TRR. The TRR of the SRT, CRT and P300 amplitude and latency in general was moderate to high. The current study suggests that response speed measures on a behavioural and neurophysiological level deserve attention as possible markers in the diagnosis and follow-up of AD.

In chapter 6 the EEG paradigms that were studied in the previous chapters were compared head-to head in order to determine which para- 
digm is most sensitive, specific and reliable in discriminating between $A D$, $\mathrm{MCl}$ and healthy controls. At present it is unclear to what extent EEG measures can be used to identify $A D$ and to monitor its progression. The aim of the present study was to determine which of the EEG paradigms are the most sensitive, specific and reliable when it comes to discriminating between patients with $A D$ or $\mathrm{MCl}$ and healthy controls. The EEG paradigms assessed as described in the 3 preceding chapters were in brief: gamma-band power during resting state, music listening, story listening and visual stimulation. During resting state, delta, theta, alpha and beta power were also analysed. The ERP's that were studied were: $40-\mathrm{Hz}$ steady-state responses, the P300 and contingent negative variation. In addition to the EEG, a simple and a choice reaction time task as well as the ADAS-cog were assessed. The results show that the response speed tasks and the ADAS-cog were most accurate and reliable in discriminating patients with $A D$ from $\mathrm{MCl}$ and healthy controls. Of the EEG paradigms studied, the $P 300$, gamma-band power during music listening, and delta power during resting state were most accurate and reliable in discriminating between patient groups. Based on the present findings we can conclude that the EEG measures studied, provide no additional value to diagnostic information. However, in the follow-up of disease progression and treatment intervention several EEG paradigms are promising as candidate biomarkers.

Thirty-seven AD patients participated in the studies described in chapters 7 and 8 and were characterized by a mean (SD) age of 72.6 (9.1); a mean (SD) MMSE score of 20.4 (4.1); and a mean (SD) GDS score of 3.5 (0.9).

In chapter 7 the association was studied between neuropsychiatric syndromes and cortical volume changes in AD. Neuropsychiatric symptoms are a common feature of Alzheimer's disease and have a negative effect on the quality of life of the patients and their caregivers. The pathogenesis or neural basis of neuropsychiatric symptoms in AD has not yet been clarified however. Studies using neuroimaging of neuropsychiatric disorders in AD have shown a large variability in the brain areas associated with individual symptoms. The accumulating evidence that neuropsychiatric symptoms rarely occur in isolation, but more often in syndromes, points to a common pathogenesis. The aim of the present study was to investigate the association between neuropsychiatric syndromes and cortical volume differences, using voxel-based morphometry (VBM). The neuropsychiatric inventory (NPI) was used to assess the presence and severity of symptoms, which were grouped in four syndromes, viz.: hyperactivity, psychosis, affective disorders and apathy. The results show that hyperactivity was associated with decreased gray matter (GM) densities in the bilateral parahippocampal cortex; in the 
left middle temporal gyrus; in the bilateral medial frontal gyrus; and in the anterior cingulate cortex. Psychosis was associated with decreased GM densities in the left medial frontal gyrus; in the right anterior cingulate; in the right caudate nucleus; and in the left middle temporal gyrus. The present study was the first to investigate the volumetric correlates of neuropsychiatric syndromes in $A D$. Future studies should be able to point out whether neuroimaging of the neuropsychiatric syndromes in $A D$ provides more consistent results than can be obtained by focusing on individual symptoms.

In chapter $\mathbf{8}$, the association was studied between apathy and cortical volume changes in Alzheimer's disease (AD). Apathy is among the most prevalent and severe neuropsychiatric symptoms in Alzheimer's disease (AD). The pathogenesis of apathy, and in particular of its sub-dimensions, has not yet been clarified. The aim of the present study was to investigate structural neuroimaging correlates of apathy while taking the following sub-dimensions into account: emotional blunting, lack of initiative and lack of interest. Gray matter (GM) volumes were determined by using voxel-based morphometry. Apathy was assessed in $37 \mathrm{AD}$ patients with the help of the Neuropsychiatric Inventory, the Apathy Evaluation Scale (AES) and the Apathy Inventory. The results showed that one sub-dimension of apathy, i.e. emotional blunting was associated with decreased GM densities in the anterior cingulate cortex; in the left caudate nucleus; in the left superior temporal gyrus; in the right middle temporal gyrus; in the right insula; and in the left lingual gyrus. Furthermore, apathy as defined by the AES was related to decreased GM densities in the left superior frontal gyrus. These data support the idea that several brain areas, including the prefrontal sub-cortical areas, are involved in the pathogenesis of apathy and stress the role of neuronal circuits in mediating the different aspects of apathy and in particular the emotional sub-dimensions. Further neuroimaging studies are needed to confirm and validate the hypoth esis that specific neuroanatomical correlates support the presence of the subdimensions of apathy and to determine its specificity across neuropsychiatric diseases.

In chapter 9 the most important findings of this thesis are discussed in a broader perspective of objective measures that assess functional and structural changes in Alzheimer's disease. The methodological aspects of this thesis are considered. From this chapter it becomes clear that future research should aim to fill the gap between behavioral, functional, structural and neurochemical changes of Alzheimer's disease in order to better understand and measure its pathological features. 
Samenvatting 

De ziekte van Alzheimer (ZvA) is de meest voorkomende vorm van dementie. De ZvA wordt gekenmerkt door progressieve achteruitgang van cognitieve functies en de aanwezigheid van neuropsychiatrische problemen. Op dit moment zijn er geen therapieën beschikbaar die de pathologische oorzaak van de ZvA beïnvloeden. Het gebrek aan effectieve therapeutische interventies vraagt om goede biologische markers (biomarkers) voor het meten van veranderingen in de cognitie, neuropathologie, morfologie en neurofysiologie die gepaard gaan met de aanwezigheid en progressie van de ZvA.

In het eerste deel van deze thesis worden verschillende parameters van het elektro-encefalogram (EEG) geëvalueerd als potentiële nieuwe biomarker voor het stellen van de diagnose, het monitoren van het ziektebeloop, en het vervolgen van de effecten van therapeutische interventies.

Het tweede deel van dit proefschrift onderzoekt het neuro-anatomische correlaat van gedragsproblemen in de ZvA.

Aan de onderzoeken van dit proefschrift participeerden een totaal van 44 Alzheimer patiënten, 20 patiënten met milde cognitieve klachten (MCl) en 20 gezonde proefpersonen.

Hoofdstuk 2 geeft een overzicht van de beschikbare literatuur die betrekking heeft op de verschillende biomarkers voor de diagnose en het volgen van het ziektebeloop van de ZvA. Het doel was om EEG maten te vergelijken met andere biomarkers, om op deze manier meer inzicht te kunnen geven in de aanvullende waarde van EEG als biomarker voor de ZvA. De waarde van verschillende EEG parameters als biomarker werd vergeleken met cognitieve markers, biochemische markers, en markers verkregen uit functionele en structurele beeldvormende technieken. De sensitiviteit, specificiteit en betrouwbaarheid van de bovengenoemde meetinstrumenten werden met elkaar vergeleken. De conclusie van dit literatuuronderzoek is dat EEG waardevolle informatie kan bieden wanneer het instrument gebruikt wordt om het ziektebeloop van ZvA te volgen of wanneer het gebruikt wordt om het effect van therapeutische interventies te evalueren. Daarnaast werd het gebrek aan informatie over de sensitiviteit, specificiteit en betrouwbaarheid van EEG parameters ter discussie gesteld.

In hoofdstuk 3 tot en met 6 werden de experimenteel verkregen gegevens over een aantal elektrofysiologische meetinstrumenten, cognitieve meetinstrumenten en gedragsmaten vergeleken tussen patiënten met de ZvA, patiënten met $\mathrm{MCl}$ en gezonde ouderen. De groep met 15 Alzheimer patiënten (11 mannen, 4 vrouwen) hadden een gemiddelde leeftijd van 75.2 (SD 6.9) en een gemiddelde Mini Mental State Examination (MMSE) score van 20.8 (SD 2.7, 17-26). De groep met $20 \mathrm{MCl}$ patiënten (12 mannen, 8 vrouwen) had een gemiddelde leeftijd van 
Functional and structural brain markers of Alzheimer's disease | Samenvatting

70.6 (SD 7.2) en een gemiddelde MMSE score van 26.3 (SD 1.6, 23-29). De groep met 20 gezonde proefpersonen (12 mannen, 8 vrouwen) had een gemiddelde leeftijd van 69.5 (SD 6.1) en een gemiddelde MMSE score van 29.3 (SD 0.8, 28-30). Alle deelnemers ondergingen de onderzoeksprocedures zoals beschreven in hoofdstuk 3 tot en met 6 . Om de test-hertest betrouwbaarheid (TRR) van de onderzoeksvariabelen te bepalen ondergingen alle deelnemers een tweede identieke testsessie een week na de eerste testsessie.

In hoofdstuk 3 werden verschillen in het gamma band EEG onderzocht tussen patiënten met ZvA, MCl patiënten en gezonde ouderen. De gamma band is de hoogste EEG frequentie $(30-100 \mathrm{~Hz})$, waarvan verondersteld wordt dat deze een belangrijke rol speelt in perceptie en het verwerken van sensorische informatie. De gamma band power werd gemeten in vier testcondities: rust, muziek luisteren, luisteren naar een verhaal en visuele stimulatie met abstracte beelden. In zijn algemeenheid toonde het EEG signaal een hoge TRR. In alle testcondities was de power van het gamma band signaal hoger in patiënten met ZvA ten opzichte van patiënten met $\mathrm{MCl}$ en gezonde proefpersonen. De resultaten van dit onderzoek suggereren dat gamma band EEG een reproduceerbare en gevoelige methode is om Alzheimer patiënten te onderscheiden van $\mathrm{MCl}$ patiënten en gezonde ouderen.

Hoofdstuk 4 beschrijft de resultaten van onderzoek naar de $40-\mathrm{Hz}$ steady-state response (SSR). De $40-\mathrm{Hz}$ SSR reflecteert vroege componenten van sensorische informatieverwerking en kan gemeten worden met EEG. In dit experiment werd de $40-\mathrm{Hz}$ SSR geïnduceerd met behulp van auditieve stimuli op met een frequentie van $40 \mathrm{~Hz}$. De resultaten tonen een hoge TRR van het 40-Hz signaal aan. De 40-Hz SSR is significant hoger in de ZvA groep vergeleken met de $\mathrm{MCl}$ groep en de controle groep. De bevindingen van dit experiment laten zien dat de $40-\mathrm{Hz}$ SSR een interessante biomarker zou kunnen zijn om het ziektebeloop van de ZvA te monitoren.

In hoofdstuk 5 werden de neurofysiologische correlaten van vertraagde snelheid van informatieverwerking in de ZvA en $\mathrm{MCl}$ onderzocht. Recent onderzoek toonde aan dat reactiesnelheid (RS) een gevoeligere indicator van verandering in het ziekteproces van de ZvA kan zijn dan het standaard meetinstrument, de Alzheimer's Disease Assessment Scale (ADAS-cog). Event related potentials (ERP's) kunnen inzicht geven in de neurofysiologische processen die betrokken zijn bij RS. In dit experiment werd RS gemeten met behulp van een simpele reactiesnelheidstaak (SRT) en een keuze reactiesnelheidstaak (CRT). Een stimulus classificatie paradigma en een contingent negatieve variantie (CNV) paradigma werden gebruikt om de ERP's te induceren. De reactietijden gemeten met de SRT en CRT maakten een significant onderscheid 
tussen de patiëntengroepen. De amplitude en latentie van P300 toonden significante verschillen tussen de groepen en een significante samenhang met de reactiesnelheid. De amplitude van CNV toonde geen significante groepsverschillen en een lage TRR. De SRT, CRT en P300 amplitude en latentie toonde een gemiddelde tot hoge TRR. De resultaten van dit experiment suggereren dat parameters van reactiesnelheid gemeten op zowel gedragsniveau als neurofysiologisch niveau meer aandacht verdienen als potentiële biomarker voor het vaststellen en vervolgen van de ziekte van Alzheimer.

In hoofdstuk 6 werden de verschillende EEG paradigma's die beschreven werden in de voorgaande hoofdstukken met elkaar vergeleken. Op dit moment is het onduidelijk in welke mate EEG paradigma's een bijdrage kunnen leveren als meetinstrument om het ziekte beloop te monitoren. Het doel van dit onderzoek was te bepalen welke van de beschreven paradigma's het meest sensitief, specifiek en betrouwbaar zijn in het maken van een onderscheid tussen ZvA patiënten, $\mathrm{MCl}$ patiënten en gezonde ouderen. De EEG paradigma's die in de voorgaande hoofdstukken werden onderzocht waren: gamma band power tijdens rust, muziek luisteren, verhaal luisteren, en visuele stimulatie. De EEG data die werden verzameld tijdens rust werden ook geanalyseerd voor verschillen in de delta band (1-4 Hz), theta band (4-8 Hz), alfa band (8-13 Hz) en beta band (13-30 Hz). De onderzochte ERP's waren $40-\mathrm{Hz}$ SSR, P300 en CNV. Aanvullend op de EEG parameters werden ook de SRT, CRT en ADAS-cog onderzocht. De resultaten tonen aan dat de reactiesnelheidstaken en de ADAS-cog het meest accuraat en betrouwbaar zijn in het discrimineren tussen patiënten met ZvA en $\mathrm{MCl}$ en gezonde controle personen. Van de EEG parameters waren de P300, gamma band tijdens muziek luisteren en delta band tijdens rust het meest sensitief, specifiek en betrouwbaar in het discrimineren tussen $\mathrm{ZvA}, \mathrm{MCl}$ en gezonde ouderen. Gebaseerd op de bevindingen in dit hoofdstuk en de voorgaande hoofdstukken kan geconcludeerd worden dat EEG geen aanvullende waarde heeft in de diagnostiek van de ziekte van Alzheimer. Daarnaast toont dit onderzoek aan dat EEG een biomarker is die mogelijk kan bijdragen aan het monitoren van het ziekte beloop en van waarde kan zijn in het meten van therapeutische effecten in de ziekte van Alzheimer.

Aan de onderzoeken die beschreven worden in hoofdstuk 7 en 8 participeerden 37 patiënten met de ziekte van Alzheimer. Deze patiënten hadden een gemiddelde leeftijd van 72.6 (SD 9.1) een gemiddelde MMSE score van 20.4 (SD 4.1) en een gemiddelde Global Deterioration Scale (GDS) van 3.5 (SD 0.9).

In hoofdstuk 7 werd de associatie tussen neuropsychiatrische stoornissen en corticale hersenvolumen onderzocht in de ZvA. Neuropsychiatrische 
stoornissen zijn een veel voorkomende complicatie van de ZvA. Deze stoornissen hebben een ernstige negatieve invloed op de kwaliteit van leven van de patiënten, hun familie en verzorgers. De pathogenese van neuropsychiatrische stoornissen in ZvA is echter nog niet duidelijk. Beeldvormende onderzoeken hebben een verbinding gelegd tussen veranderingen in verschillende hersengebieden en de aanwezigheid van individuele neuropsychiatrische symptomen. Recente onderzoeken wijzen erop dat neuropsychiatrische stoornissen zelden solitair voorkomen maar juist vaak in clusters van symptomen, de zogenaamde syndromen. De samenhang van symptomen in syndromen wijst op een overeenkomstige pathogenese. Het doel van dit onderzoek was de samenhang tussen de aanwezigheid en de ernst van neuropsychiatrische syndromen en veranderingen van het corticale hersenvolume te onderzoeken met behulp van voxel-based morphometry (VBM). De Neuropsychiatric Inventory (NPI) werd gebruikt om de aanwezigheid en de ernst van neuropsychiatrische symptomen te meten. Deze symptomen werden geclusterd in de volgende vier syndromen: hyperactiviteit, psychose, affectieve stoornissen en apathie. De resultaten tonen een associatie tussen hyperactiviteit en een verminderd grijze stof volume in de bilaterale parahippocampale cortex, de linker mediale gyrus temporalis, de linker mediale gyrus frontalis en de anterior cingulate cortex. Het syndroom psychose is geassocieerd met een verminderd grijze stof volume in de linker mediale gyrus frontalis, de linker gyrus temporalis, de rechter anterior cingulate cortex en de rechter nucleus caudatus. Dit onderzoek was het eerste dat het volumetrisch correlaat van neuropsychiatrische syndromen in de ZvA onderzocht. Toekomstige beeldvormende onderzoeken zouden moeten kunnen uitsluiten of het onderzoeken van neuropsychiatrische syndromen meer consistente resultaten oplevert dan het gebruik van individuele neuropsychiatrische symptomen.

In hoofdstuk 8 werd de associatie tussen apathie en corticale hersenvolume veranderingen onderzocht in de ZvA. Apathie is een van de meest voorkomende en meest ernstige neuropsychiatrische complicatie van de ZvA. De pathogenese van apathie en de sub-domeinen van apathie is tot op heden onbekend. Het doel van dit onderzoek was meer inzicht te verkrijgen in het neuraal correlaat van apathie en de volgende specifieke sub-domeinen: emotionele afvlakking, gebrek aan initiatiefname en gebrek aan interesse. Veranderingen van grijze stof volumen werden gemeten met behulp van VBM. De aanwezigheid en de ernst van apathie werd gemeten met de volgende meetinstrumenten: Neuropsychiatric Inventory (NPI), Apathie Evaluation Scale (AES) en de Apathie Inventory (AI). De resultaten tonen dat emotionele afvlakking geassocieerd is met een verminderd grijze stof volume in de anterior 
cingulate cortex, linker nucleus caudatus, linker gyrus temporalis superior, de rechter mediale gyrus temporalis, de linker insula en de linker gyrus lingualis. Daarnaast was de ernst van apathie gemeten met de AES geassocieerd met een verminderd grijze stof volume in de linker gyrus frontalis superior. Deze resultaten ondersteunen de hypothese dat verschillende hersengebieden die ook subcorticale prefrontaal gebieden omvatten, betrokken zijn in de pathogenese van apathie. De huidige bevindingen bevestigen de rol van neurale circuits in de verschillende aspecten van apathie en meer specifiek in de emotionele sub-domeinen. Toekomstig onderzoek zou de rol van specifieke neurale circuits in het ontstaan van apathie moeten bevestigen en valideren.

In hoofdstuk 9 werden de belangrijkste bevindingen van dit proefschrift ter discussie gesteld en in een breder perspectief geplaatst. Daarnaast werden verschillende methodologische aspecten in overweging genomen. Deze discussie wijst op het belang van toekomstig multidimensioneel onderzoek. Een multidimensionele benadering zou het gat tussen verschillende onderzoeksdomeinen binnen de ziekte van Alzheimer moeten overbruggen om deze ziekte beter te leren begrijpen en meten. 



$$
\text { References }
$$



Consensus report of the Working Group on: "Molecular and Biochemical Marker of Alzheimer's Disease". The Ronald and Nancy Reagan Research Institute of the Alzheimer's Association and the National Institute on Aging Working Group. Neurobiol Aging 1998; 19: 109-16.

Aalten P, de Vugt ME, Jaspers N, Jolles J, Verhey FR. The course of neuropsychiatric symptoms in dementia. Part I: findings from the two-year longitudinal Maasbed study. Int J Geriatr Psychiatry 2005a; 20: 523-30.

Aalten P, de Vugt ME, Jaspers N, Jolles J, Verhey FR. The course of neuropsychiatric symptoms in dementia. Part II: relationships among behavioural sub-syndromes and the influence of clinical variables. Int J Geriatr Psychiatry 2005b; 20: 531-6.

Aalten P, Jolles J, de Vugt ME, Verhey FR. The influence of neuropsychological functioning on neuropsychiatric problems in dementia. J Neuropsychiatry Clin Neurosci 2007a; 19: 50-6.

Aalten P, Verhey FR, Boziki M, Brugnolo A, Bullock R, Byrne EJ, et al. Consistency of neuropsychiatric syndromes across dementias: results from the European Alzheimer Disease Consortium. Part II. Dement Geriatr Cogn Disord 2008; 25: 1-8.

Aalten P, Verhey FR, Boziki M, Bullock R, Byrne EJ, Camus V, et al. Neuropsychiatric syndromes in dementia. Results from the European Alzheimer Disease Consortium: part I. Dement Geriatr Cogn Disord 2007b; 24: 457-63.

Adler G, Brassen S, Chwalek K, Dieter B, Teufel M. Prediction of treatment response to rivastigmine in Alzheimer's dementia. J Neurol Neurosurg Psychiatry 2004; 75: 292-4.

Ahveninen J, Jaaskelainen IP, Kaakkola S, Tiitinen H, Pekkonen E. Aging and cholinergic modulation of the transient magnetic $40-\mathrm{Hz}$ auditory response. Neuroimage 2002; 15: 153-8.

Ahveninen J, Tiitinen H, Hirvonen J, Pekkonen E, Huttunen J, Kaakkola S, et al. Scopolamine augments transient auditory $40-\mathrm{hz}$ magnetic response in humans. Neurosci Lett 1999; 277: 115-8.

American Psychiatric Association. Diagnostic and Statistical Manual of Mental Disorders (DSM-4). Washington, DC, 1994.

Andreasen N, Blennow K. CSF biomarkers for mild cognitive impairment and early Alzheimer's disease. Clin Neurol Neurosurg 2005; 107: 165-73.

Andreasen N, Minthon L, Clarberg A, Davidsson P, Gottfries J, Vanmechelen E, et al. Sensitivity, specificity, and stability of CSF-tau in AD in a community-based patient sample. Neurology 1999; 53: 1488-94.

Apostolova LG, Akopyan GG, Partiali N, Steiner CA, Dutton RA, Hayashi KM, et al. Structural correlates of apathy in Alzheimer's disease. Dement Geriatr Cogn Disord 2007; 24: 91-7.

Ashburner J, Friston KJ. Voxel-based morphometry--the methods. Neuroimage 2000; 11: 805-21. 
Atiya M, Hyman BT, Albert MS, Killiany R. Structural magnetic resonance imaging in established and prodromal Alzheimer disease: a review. Alzheimer Dis Assoc Disord 2003; 17: 177-95.

Babiloni C, Cassetta E, Dal Forno G, Del Percio C, Ferreri F, Ferri R, et al. Donepezil effects on sources of cortical rhythms in mild Alzheimer's disease: Responders vs. Non-Responders. Neuroimage 2006; 31: 1650-65.

Babiloni C, Ferri R, Moretti DV, Strambi A, Binetti G, Dal Forno G, et al. Abnormal fronto-parietal coupling of brain rhythms in mild Alzheimer's disease: a multicentric EEG study. Eur J Neurosci 2004; 19: 2583-90.

Backman L, Jones S, Berger AK, Laukka EJ, Small BJ. Multiple cognitive deficits during the transition to Alzheimer's disease. J Intern Med 2004; 256: 195-204.

Ballard CG, Margallo-Lana M, Fossey J, Reichelt K, Myint P, Potkins D, et al. A 1-year follow-up study of behavioral and psychological symptoms in dementia among people in care environments. J Clin Psychiatry 2001; 62: 631-6.

Barnes J, Scahill RI, Boyes RG, Frost C, Lewis EB, Rossor CL, et al. Differentiating AD from aging using semiautomated measurement of hippocampal atrophy rates. Neuroimage 2004; 23: 574-81.

Basar E, Basar-Eroglu C, Karakas S, Schurmann M. Gamma, alpha, delta, and theta oscillations govern cognitive processes. Int J Psychophysiol 2001; 39: 241-8.

Bashore TR, Osman A, Heffley EF, 3rd. Mental slowing in elderly persons: a cognitive psychophysiological analysis. Psychol Aging 1989; 4: 235-44.

Bellgrove MA, Phillips JG, Bradshaw JL, Hall KA, Presnell I, Hecht H. Response programming in dementia of the Alzheimer type: a kinematic analysis. Neuropsychologia 1997; 35: 229-40.

Bennys K, Portet F, Touchon J, Rondouin G. Diagnostic value of event-related evoked potentials N200 and P300 subcomponents in early diagnosis of Alzheimer's disease and mild cognitive impairment. J Clin Neurophysiol 2007; 24: 405-12.

Benoit M, Clairet S, Koulibaly PM, Darcourt J, Robert PH. Brain perfusion correlates of the apathy inventory dimensions of Alzheimer's disease. Int J Geriatr Psychiatry 2004; 19: 864-9.

Benoit M, Dygai, I., Migneco, O., Robert, P. H., Bertogliati, C., Darcourt, J., Benoliel, J., Aubin-Brunet, V., Pringuey, D. Behavioral and psychological symptoms in Alzheimer's disease. Relation between apathy and regional cerebral perfusion. Dement Geriatr Cogn Disord 1999; 10: 511-7.

Benoit M, Koulibaly PM, Migneco O, Darcourt J, Pringuey DJ, Robert PH. Brain perfusion in Alzheimer's disease with and without apathy: a SPECT study with statistical parametric mapping analysis. Psychiatry Res 2002; 114: 103-11. 
Berendse HW, Verbunt JP, Scheltens P, van Dijk BW, Jonkman EJ. Magnetoencephalographic analysis of cortical activity in Alzheimer's disease: a pilot study. Clin Neurophysiol 2000; 111: 604-12.

Blennow K, Hampel H. CSF markers for incipient Alzheimer's disease. Lancet Neurol 2003; 2: 605-13.

Blennow K, Wallin A, Agren H, Spenger C, Siegfried J, Vanmechelen E. Tau protein in cerebrospinal fluid: a biochemical marker for axonal degeneration in Alzheimer disease? Mol Chem Neuropathol 1995; 26: 231-45.

Blessed $\mathrm{G}$, Tomlinson BE, Roth $\mathrm{M}$. The association between quantitative measures of dementia and of senile change in the cerebral grey matter of elderly subjects. $\mathrm{Br} \mathrm{J}$ Psychiatry 1968; 114: 797-811.

Bookstein FL. "Voxel-based morphometry" should not be used with imperfectly registered images. Neuroimage 2001; 14: 1454-62.

Bottino CM, Castro CC, Gomes RL, Buchpiguel CA, Marchetti RL, Neto MR. Volumetric MRI measurements can differentiate Alzheimer's disease, mild cognitive impairment, and normal aging. Int Psychogeriatr 2002; 14: 59-72.

Boutros N, Torello MW, Burns EM, Wu SS, Nasrallah HA. Evoked potentials in subjects at risk for Alzheimer's disease. Psychiatry Res 1995; 57: 57-63.

Boyle PA, Malloy PF. Treating apathy in Alzheimer's disease. Dement Geriatr Cogn Disord 2004; 17: 91-9.

Bozzola FG, Gorelick, P.B., Freels, S. Personality changes in Alzheimer's Disease. Archives of Neurology 1992; 49: 297-300.

Braak H, Braak A. Staging Of Alzheimer's Disease-Related Neurofibrillary Changes. Neurobiology of Aging 1995; 16: 271-284.

Braak H, Braak E. Neuropathological stageing of Alzheimer-related changes. Acta Neuropathol (Berl) 1991; 82: 239-59.

Brand N, Jolles J. Learning and retrieval rate of words presented auditorily and visually. J Gen Psychol 1985; 112: 201-10.

Bruen PD, McGeown WJ, Shanks MF, Venneri A. Neuroanatomical correlates of neuropsychiatric symptoms in Alzheimer's disease. Brain 2008a.

Bruen PD, McGeown WJ, Shanks MF, Venneri A. Neuroanatomical correlates of neuropsychiatric symptoms in Alzheimer's disease. Brain 2008b; 131: 2455-63.

Brunia $\mathrm{CH}$, Vingerhoets AJ. CNV and EMG preceding a plantar flexion of the foot. Biol Psychol 1980; 11: 181-91.

Brunia $\mathrm{CH}$, Vingerhoets AJ. Opposite hemisphere differences in movement related potentials preceding foot and finger flexions. Biol Psychol 1981; 13: 261-9.

Buerger K, Teipel SJ, Zinkowski R, Blennow K, Arai H, Engel R, et al. CSF tau protein phosphorylated at threonine 231 correlates with cognitive decline in $\mathrm{MCl}$ subjects. Neurology 2002; 59: 627-9. 
Bullock R, Dengiz A. Cognitive performance in patients with Alzheimer's disease receiving cholinesterase inhibitors for up to 5 years. Int J Clin Pract 2005; 59: 817-22.

Busatto GF, Garrido GE, Almeida OP, Castro CC, Camargo CH, Cid CG, et al. A voxelbased morphometry study of temporal lobe gray matter reductions in Alzheimer's disease. Neurobiol Aging 2003; 24: 221-31.

Cabranes JA, De Juan R, Encinas M, Marcos A, Gil P, Fernandez C, et al. Relevance of functional neuroimaging in the progression of mild cognitive impairment. Neurol Res 2004; 26: 496-501.

Camarda R, Camarda C, Monastero R, Grimaldi S, Camarda LK, Pipia C, et al. Movements execution in amnestic mild cognitive impairment and Alzheimer's disease. Behav Neurol 2007; 18: 135-42.

Cancelli I, Cadore IP, Merlino G, Valentinis L, Moratti U, Bergonzi P, et al. Sensory gating deficit assessed by $\mathrm{P} 50 / \mathrm{Pb}$ middle latency event related potential in Alzheimer's disease. J Clin Neurophysiol 2006; 23: 421-5.

Caramelli P, Chaves ML, Engelhardt E, Machado JC, Schultz RR, Vale FA, et al. Effects of galantamine on attention and memory in Alzheimer's disease measured by computerized neuropsychological tests: results of the Brazilian Multi-Center Galantamine Study (GAL-BRA-01). Arq Neuropsiquiatr 2004; 62: 379-84.

Chen P, Ratcliff G, Belle SH, Cauley JA, DeKosky ST, Ganguli M. Patterns of cognitive decline in presymptomatic Alzheimer disease: a prospective community study. Arch Gen Psychiatry 2001; 58: 853-8.

Chetelat G, Landeau B, Eustache F, Mezenge F, Viader F, de la Sayette V, et al. Using voxel-based morphometry to map the structural changes associated with rapid conversion in MCl: a longitudinal MRI study. Neuroimage 2005; 27: 934-46.

Clarke DE, Van Reekum R, Patel J, Simard M, Gomez E, Streiner DL. An appraisal of the psychometric properties of the Clinician version of the Apathy Evaluation Scale (AES-C). Int J Methods Psychiatr Res 2007; 16: 97-110.

Craig AH, Cummings, J.L., Fairbanks, L., Itti, L., Miller, B.L., Li, J., Mena, I. Cerebral blood flow correlates of apathy in Alzheimer Disease. Archives of Neurology 1996; 53: 1116-1120.

Csernansky JG, Hamstra J, Wang L, McKeel D, Price JL, Gado M, et al. Correlations between antemortem hippocampal volume and postmortem neuropathology in $A D$ subjects. Alzheimer Dis Assoc Disord 2004; 18: 190-5.

Cummings JL. The Neuropsychiatric Inventory: assessing psychopathology in dementia patients. Neurology 1997; 48: S10-6.

Cummings JL. Use of cholinesterase inhibitors in clinical practice: evidence-based recommendations. Am J Geriatr Psychiatry 2003; 11: 131-45.

Cummings JL, McPherson S. Neuropsychiatric assessment of Alzheimer's disease and related dementias. Aging (Milano) 2001; 13: 240-6. 
Cummings JL, Mega M, Gray K, Rosenberg-Thompson S, Carusi DA, Gornbein J. The Neuropsychiatric Inventory: comprehensive assessment of psychopathology in dementia. Neurology 1994; 44: 2308-14.

Cummings JL, Mega, M., Gray, K., Rosenberg-Thompson, S., Carusi, D.A., Gornbein, J. The Neuropsychiatric Inventory: Comprehensive assessment of psychopathology in dementia. Neurology 1994; 44: 2308-2314.

D'Esposito M, Deouell LY, Gazzaley A. Alterations in the BOLD fMRI signal with ageing and disease: a challenge for neuroimaging. Nat Rev Neurosci 2003; 4: 863-72.

David R, Koulibaly M, Benoit M, Garcia R, Caci H, Darcourt J, et al. Striatal dopamine transporter levels correlate with apathy in neurodegenerative diseases A SPECT study with partial volume effect correction. Clin Neurol Neurosurg 2008; 110: 19-24.

De Gruttola VG, Clax P, DeMets DL, Downing GJ, Ellenberg SS, Friedman L, et al. Considerations in the evaluation of surrogate endpoints in clinical trials. summary of a National Institutes of Health workshop. Control Clin Trials 2001; 22: 485-502.

De Jager CA, Hogervorst E, Combrinck M, Budge MM. Sensitivity and specificity of neuropsychological tests for mild cognitive impairment, vascular cognitive impairment and Alzheimer's disease. Psychol Med 2003; 33: 1039-50.

de Vugt ME, Riedijk SR, Aalten P, Tibben A, van Swieten JC, Verhey FR. Impact of behavioural problems on spousal caregivers: a comparison between Alzheimer's disease and frontotemporal dementia. Dement Geriatr Cogn Disord 2006; 22: 35-41.

Delbeuck X, Van der Linden M, Collette F. Alzheimer's disease as a disconnection syndrome? Neuropsychol Rev 2003; 13: 79-92.

Derix MM, Hofstede AB, Teunisse S, Hijdra A, Walstra GJ, Weinstein HC, et al. [CAMDEX-N: the Dutch version of the Cambridge Examination for Mental Disorders of the Elderly with automatic data processing]. Tijdschr Gerontol Geriatr 1991; 22: 143-50.

Derix MM, Hofstede, A. B., Teunisse, S., Hijdra, A., Walstra, G. J., Weinstein, H. C., van Gool, W. A. [CAMDEX-N: the Dutch version of the Cambridge Examination for Mental Disorders of the Elderly with automatic data processing]. Tijdschrift voor Gerontologie en Geriatrie 1991; 22: 143-50.

Di Lazzaro V, Oliviero A, Pilato F, Saturno E, Dileone M, Marra C, et al. Motor cortex hyperexcitability to transcranial magnetic stimulation in Alzheimer's disease. J Neurol Neurosurg Psychiatry 2004; 75: 555-9.

Dimoska A, Johnstone SJ, Barry RJ. The auditory-evoked N2 and P3 components in the stop-signal task: indices of inhibition, response-conflict or error-detection? Brain Cogn 2006; 62: 98-112. 
Doody RS, Stevens JC, Beck C, Dubinsky RM, Kaye JA, Gwyther L, et al. Practice parameter: management of dementia (an evidence-based review). Report of the Quality Standards Subcommittee of the American Academy of Neurology. Neurology 2001; 56: 1154-66.

Dougall NJ, Bruggink S, Ebmeier KP. Systematic review of the diagnostic accuracy of 99mTc-HMPAO-SPECT in dementia. Am J Geriatr Psychiatry 2004; 12: 554-70.

Driver JE, Racca C, Cunningham MO, Towers SK, Davies $\mathrm{CH}$, Whittington MA, et al. Impairment of hippocampal gamma-frequency oscillations in vitro in mice overexpressing human amyloid precursor protein (APP). Eur J Neurosci 2007; 26: 1280-8.

Drzezga A, Grimmer T, Riemenschneider M, Lautenschlager N, Siebner H, Alexopoulus P, et al. Prediction of individual clinical outcome in $\mathrm{MCl}$ by means of genetic assessment and (18)F-FDG PET. J Nucl Med 2005; 46: 1625-32.

Duffy FH, Albert MS, McAnulty G. Brain electrical activity in patients with presenile and senile dementia of the Alzheimer type. Ann Neurol 1984; 16: 439-48.

Duke LM, Kaszniak AW. Executive control functions in degenerative dementias: a comparative review. Neuropsychol Rev 2000; 10: 75-99.

Ebert U, Kirch W. Scopolamine model of dementia: electroencephalogram findings and cognitive performance. Eur J Clin Invest 1998; 28: 944-9.

Encinas M, De Juan R, Marcos A, Gil P, Barabash A, Fernandez C, et al. Regional cerebral blood flow assessed with 99mTc-ECD SPET as a marker of progression of mild cognitive impairment to Alzheimer's disease. Eur J Nucl Med Mol Imaging 2003; 30: 1473-80.

Engel AK, Fries P, Singer W. Dynamic predictions: oscillations and synchrony in top-down processing. Nat Rev Neurosci 2001; 2: 704-16.

Engel AK, Singer W. Temporal binding and the neural correlates of sensory awareness. Trends Cogn Sci 2001; 5: 16-25.

Farber NB, Rubin EH, Newcomer JW, Kinscherf DA, Miller JP, Morris JC, et al. Increased neocortical neurofibrillary tangle density in subjects with Alzheimer disease and psychosis. Arch Gen Psychiatry 2000; 57: 1165-73.

Fitzgibbon SP, Pope KJ, Mackenzie L, Clark CR, Willoughby JO. Cognitive tasks augment gamma EEG power. Clin Neurophysiol 2004; 115: 1802-9.

Folstein MF, Folstein SE, McHugh PR. "Mini-mental state" : A practical method for grading the cognitive state of patients for the clinician. Journal of Psychiatric Research 1975a; 12: 189-198.

Folstein MF, Folstein SE, McHugh PR. "Mini-mental state". A practical method for grading the cognitive state of patients for the clinician. J Psychiatr Res 1975b; 12: 189-98.

Folstein N, Folstein S, McHugh P. Mini-mental state: a practical method for grading the cognitive state of patients for clinician. Journal of Psychiatry Research 1975c; 12: 189-198. 
Fox NC, Schott JM. Imaging cerebral atrophy: normal ageing to Alzheimer's disease. Lancet 2004; 363: 392-4.

Fox NC, Warrington EK, Rossor MN. Serial magnetic resonance imaging of cerebral atrophy in preclinical Alzheimer's disease. Lancet 1999; 353: 2125.

Francis PT. Glutamatergic systems in Alzheimer's disease. Int J Geriatr Psychiatry 2003; 18: S15-21.

Francis PT. The interplay of neurotransmitters in Alzheimer's disease. CNS Spectr 2005; 10: 6-9.

Frisoni GB, Rozzini L, Gozzetti A, Binetti G, Zanetti O, Bianchetti A, et al. Behavioral syndromes in Alzheimer's disease: description and correlates. Dement Geriatr Cogn Disord 1999; 10: 130-8.

Frodl T, Hampel H, Juckel G, Burger K, Padberg F, Engel RR, et al. Value of eventrelated $\mathrm{P} 300$ subcomponents in the clinical diagnosis of mild cognitive impairment and Alzheimer's Disease. Psychophysiology 2002; 39: 175-81.

Frund I, Schadow J, Busch NA, Korner U, Herrmann CS. Evoked gamma oscillations in human scalp EEG are test-retest reliable. Clin Neurophysiol 2007; 118: 221-7.

Gaeta H, Friedman D, Ritter W, Cheng J. Changes in sensitivity to stimulus deviance in Alzheimer's disease: an ERP perspective. Neuroreport 1999; 10: 281-7.

Galambos R, Makeig S, Talmachoff PJ. A 40-Hz auditory potential recorded from the human scalp. Proc Natl Acad Sci U S A 1981; 78: 2643-7.

Garcia-Alloza M, Tsang SW, Gil-Bea FJ, Francis PT, Lai MK, Marcos B, et al. Involvement of the GABAergic system in depressive symptoms of Alzheimer's disease. Neurobiol Aging 2006; 27: 1110-7.

Gauthier S, Wirth Y, Mobius HJ. Effects of memantine on behavioural symptoms in Alzheimer's disease patients: an analysis of the Neuropsychiatric Inventory (NPI) data of two randomised, controlled studies. Int J Geriatr Psychiatry 2005; 20: 459-64.

Gee J, Ding L, Xie Z, Lin M, DeVita C, Grossman M. Alzheimer's disease and frontotemporal dementia exhibit distinct atrophy-behavior correlates: a computer-assisted imaging study. Acad Radiol 2003; 10: 1392-401.

Geuze E, Vermetten E, Bremner JD. MR-based in vivo hippocampal volumetrics: 2. Findings in neuropsychiatric disorders. Mol Psychiatry 2005; 10: 160-84.

Gironell A, Garcia-Sanchez C, Estevez-Gonzalez A, Boltes A, Kulisevsky J. Usefulness of p300 in subjective memory complaints: a prospective study. J Clin Neurophysiol 2005; 22: 279-84

Golob EJ, Irimajiri R, Starr A. Auditory cortical activity in amnestic mild cognitive impairment: relationship to subtype and conversion to dementia. Brain 2007; 130: 740-52.

Golob EJ, Johnson JK, Starr A. Auditory event-related potentials during target detection are abnormal in mild cognitive impairment. Clin Neurophysiol 2002; 113: 151-61. 
Golob EJ, Miranda GG, Johnson JK, Starr A. Sensory cortical interactions in aging, mild cognitive impairment, and Alzheimer's disease. Neurobiol Aging 2001; 22: 755-63.

Golob EJ, Starr A. Effects of stimulus sequence on event-related potentials and reaction time during target detection in Alzheimer's disease. Clin Neurophysiol 2000; 111: 1438-49.

Goncharova, II, Dickerson BC, Stoub TR, deToledo-Morrell L. MRI of human entorhinal cortex: a reliable protocol for volumetric measurement. Neurobiol Aging 2001; 22: 737-45.

Good CD, Johnsrude IS, Ashburner J, Henson RN, Friston KJ, Frackowiak RS. A voxelbased morphometric study of ageing in 465 normal adult human brains. Neuroimage 2001; 14: 21-36.

Goodin DS, Aminoff MJ. Evaluation of dementia by event-related potentials. J Clin Neurophysiol 1992; 9: 521-5.

Gordon B, Carson K. The basis for choice reaction time slowing in Alzheimer's disease. Brain Cogn 1990; 13: 148-66.

Gosche KM, Mortimer JA, Smith CD, Markesbery WR, Snowdon DA. Hippocampal volume as an index of Alzheimer neuropathology: findings from the Nun Study. Neurology 2002; 58: 1476-82.

Gutschalk A, Mase R, Roth R, Ille N, Rupp A, Hahnel S, et al. Deconvolution of $40 \mathrm{~Hz}$ steady-state fields reveals two overlapping source activities of the human auditory cortex. Clin Neurophysiol 1999; 110: 856-68.

Haagh SA, Brunia CH. Anticipatory response-relevant muscle activity, CNV amplitude and simple reaction time. Electroencephalogr Clin Neurophysiol 1985; 61: 30-9.

Haass C, Schlossmacher MG, Hung AY, Vigo-Pelfrey C, Mellon A, Ostaszewski BL, et al. Amyloid beta-peptide is produced by cultured cells during normal metabolism. Nature 1992; 359: 322-5.

Hachinski VC, Lassen NA, Marshall J. Multi-infarct dementia. A cause of mental deterioration in the elderly. Lancet 1974; 2: 207-10.

Hamilton M. A rating scale for depression. J Neurol Neurosurg Psychiatry 1960; 23: $56-62$.

Hampel H, Buerger K, Kohnken R, Teipel SJ, Zinkowski R, Moeller HJ, et al. Tracking of Alzheimer's disease progression with cerebrospinal fluid tau protein phosphorylated at threonine 231. Ann Neurol 2001; 49: 545-6.

Hampel H, Mitchell A, Blennow K, Frank RA, Brettschneider S, Weller L, et al. Core biological marker candidates of Alzheimer's disease - perspectives for diagnosis, prediction of outcome and reflection of biological activity. J Neural Transm 2004; 111: 247-72. 
Hasselmo ME, Fehlau BP. Differences in time course of ACh and GABA modulation of excitatory synaptic potentials in slices of rat hippocampus. J Neurophysiol 2001; 86: $1792-802$.

Herrmann CS, Demiralp T. Human EEG gamma oscillations in neuropsychiatric disorders. Clin Neurophysiol 2005; 116: 2719-33.

Herrmann CS, Munk MH, Engel AK. Cognitive functions of gamma-band activity: memory match and utilization. Trends Cogn Sci 2004; 8: 347-55.

Herrmann N, Rabheru K, Wang J, Binder C. Galantamine treatment of problematic behavior in Alzheimer disease: post-hoc analysis of pooled data from three large trials. Am J Geriatr Psychiatry 2005; 13: 527-34.

Hesse C, Rosengren L, Andreasen N, Davidsson P, Vanderstichele H, Vanmechelen E, et al. Transient increase in total tau but not phospho-tau in human cerebrospinal fluid after acute stroke. Neurosci Lett 2001; 297: 187-90.

Hirata Y, Matsuda H, Nemoto K, Ohnishi T, Hirao K, Yamashita F, et al. Voxel-based morphometry to discriminate early Alzheimer's disease from controls. Neurosci Lett 2005; 382: 269-74.

Hogervorst E, Bandelow S, Combrinck M, Irani SR, Smith AD. The validity and reliability of 6 sets of clinical criteria to classify Alzheimer's disease and vascular dementia in cases confirmed post-mortem: added value of a decision tree approach. Dement Geriatr Cogn Disord 2003; 16: 170-80.

Holm A, Ranta-aho PO, Sallinen M, Karjalainen PA, Muller K. Relationship of P300 single-trial responses with reaction time and preceding stimulus sequence. Int J Psychophysiol 2006; 61: 244-52.

Holschneider DP, Waite JJ, Leuchter AF, Walton NY, Scremin OU. Changes in electrocortical power and coherence in response to the selective cholinergic immunotoxin 192 IgG-saporin. Exp Brain Res 1999; 126: 270-80.

Holthoff VA, Beuthien-Baumann B, Kalbe E, Ludecke S, Lenz O, Zundorf G, et al. Regional cerebral metabolism in early Alzheimer's disease with clinically significant apathy or depression. Biol Psychiatry 2005; 57: 412-21.

Houx PJ, Jolles J, Vreeling FW. Stroop interference: aging effects assessed with the Stroop Color-Word Test. Exp Aging Res 1993; 19: 209-24.

Huang C, Wahlund LO, Dierks T, Julin P, Winblad B, Jelic B. Discrimination of Alzheimer's disease and mild cognitive impairment by equivalent EEG sources: a cross-sectional and longitudinal study. Clinical Neurophysiology 2000; 111: 1961-1967.

Huang C, Wahlund LO, Svensson L, Winblad B, Julin P. Cingulate cortex hypoperfusion predicts Alzheimer's disease in mild cognitive impairment. BMC Neurol 2002; 2: 9.

Ito J, Yamao S, Fukuda H, Mimori Y, Nakamura S. The P300 event-related potentials in dementia of the Alzheimer type. Correlations between P300 and monoamine metabolites. Electroencephalogr Clin Neurophysiol 1990; 77: 174-8. 
Jaaskelainen IP, Hirvonen J, Saher M, Pekkonen E, Sillanaukee P, Naatanen R, et al. Benzodiazepine temazepam suppresses the transient auditory $40-\mathrm{Hz}$ response amplitude in humans. Neurosci Lett 1999; 268: 105-7.

Jack CR, Jr., Lowe VJ, Senjem ML, Weigand SD, Kemp BJ, Shiung MM, et al. 11C PiB and structural MRI provide complementary information in imaging of Alzheimer's disease and amnestic mild cognitive impairment. Brain 2008; 131: 665-80.

Jacob TC, Moss SJ, Jurd R. GABA(A) receptor trafficking and its role in the dynamic modulation of neuronal inhibition. Nat Rev Neurosci 2008; 9: 331-43.

Jagust W, Reed B, Mungas D, Ellis W, Decarli C. What does fluorodeoxyglucose PET imaging add to a clinical diagnosis of dementia? Neurology 2007; 69: 871-7.

Jelic V, Johansson SE, Almkvist O, Shigeta M, Julin P, Nordberg A, et al. Quantitative electroencephalography in mild cognitive impairment: longitudinal changes and possible prediction of Alzheimer's disease. Neurobiol Aging 2000; 21: 533-40.

Jelic V, Julin P, Shigeta M, Nordberg A, Lannfelt L, Winblad B, et al. Apolipoprotein E epsilon4 allele decreases functional connectivity in Alzheimer's disease as measured by EEG coherence. J Neurol Neurosurg Psychiatry 1997; 63: 59-65.

Jelic V, Shigeta M, Julin P, Almkvist O, Winblad B, Wahlund LO. Quantitative electroencephalography power and coherence in Alzheimer's disease and mild cognitive impairment. Dementia 1996; 7: 314-23.

Jeong J. EEG dynamics in patients with Alzheimer's disease. Clin Neurophysiol 2004; 115: 1490-505.

Jessen F, Kucharski C, Fries T, Papassotiropoulos A, Hoenig K, Maier W, et al. Sensory gating deficit expressed by a disturbed suppression of the P50 event-related potential in patients with Alzheimer's disease. Am J Psychiatry 2001; 158: 1319-21.

Joliot M, Ribary U, Llinas R. Human oscillatory brain activity near $40 \mathrm{~Hz}$ coexists with cognitive temporal binding. Proc Natl Acad Sci U S A 1994; 91: 11748-51.

Jonsson L, Eriksdotter Jonhagen M, Kilander L, Soininen H, Hallikainen M, Waldemar G, et al. Determinants of costs of care for patients with Alzheimer's disease. Int J Geriatr Psychiatry 2006; 21: 449-59.

Kaiser J, Lutzenberger W. Induced gamma-band activity and human brain function. Neuroscientist 2003; 9: 475-84.

Kaiser J, Lutzenberger W. Human gamma-band activity: a window to cognitive processing. Neuroreport 2005; 16: 207-11.

Kantarci K, Jack CR, Jr. Quantitative magnetic resonance techniques as surrogate markers of Alzheimer's disease. NeuroRx 2004; 1: 196-205.

Kapaki E, Paraskevas GP, Zalonis I, Zournas C. CSF tau protein and beta-amyloid (142) in Alzheimer's disease diagnosis: discrimination from normal ageing and other dementias in the Greek population. Eur J Neurol 2003; 10: 119-28. 
Karakas S, Basar E. Early gamma response is sensory in origin: a conclusion based on cross-comparison of results from multiple experimental paradigms. Int J Psychophysiol 1998; 31: 13-31.

Karas G, Sluimer J, Goekoop R, van der Flier W, Rombouts S, Vrenken H, et al. Amnestic Mild Cognitive Impairment: Structural MR Imaging Findings Predictive of Conversion to Alzheimer Disease. AJNR Am J Neuroradiol 2008a.

Karas G, Sluimer J, Goekoop R, van der Flier W, Rombouts SA, Vrenken H, et al. Amnestic mild cognitive impairment: structural MR imaging findings predictive of conversion to Alzheimer disease. AJNR Am J Neuroradiol 2008b; 29: 944-9.

Karas GB, Burton EJ, Rombouts SA, van Schijndel RA, O'Brien JT, Scheltens P, et al. A comprehensive study of gray matter loss in patients with Alzheimer's disease using optimized voxel-based morphometry. Neuroimage 2003; 18: 895-907.

Karas GB, Scheltens P, Rombouts SA, Visser PJ, van Schijndel RA, Fox NC, et al. Global and local gray matter loss in mild cognitive impairment and Alzheimer's disease. Neuroimage 2004; 23: 708-16.

Kat MG, de Jonghe JF, Aalten P, Kalisvaart CJ, Droes RM, Verhey FR. [Neuropsychiatric symptoms of dementia: psychometric aspects of the Dutch Neuropsychiatric Inventory (NPI)]. Tijdschr Gerontol Geriatr 2002a; 33: 150-5.

Kat MG, de Jonghe JF, Aalten P, Kalisvaart CJ, Droes RM, Verhey FR. [Neuropsychiatric symptoms of dementia: psychometric aspects of the Dutch Neuropsychiatric Inventory (NPI)]. Tijdschr Gerontol Geriatr 2002b; 33: 150-5.

Katada E, Sato K, Sawaki A, Dohi Y, Ueda R, Ojika K. Long-term effects of donepezil on P300 auditory event-related potentials in patients with Alzheimer's disease. J Geriatr Psychiatry Neurol 2003; 16: 39-43.

Kazmerski VA, Friedman D, Ritter W. Mismatch negativity during attend and ignore conditions in Alzheimer's disease. Biol Psychiatry 1997; 42: 382-402.

Kemppainen NM, Aalto S, Wilson IA, Nagren K, Helin S, Bruck A, et al. PET amyloid ligand [11C]PIB uptake is increased in mild cognitive impairment. Neurology 2007; 68: 1603-6.

Kikuchi M, Wada Y, Koshino Y, Nanbu Y, Hashimoto T. Effects of scopolamine on interhemispheric EEG coherence in healthy subjects: analysis during rest and photic stimulation. Clin Electroencephalogr 2000; 31: 109-15.

Kikuchi M, Wada Y, Nanbu Y, Nakajima A, Tachibana H, Takeda T, et al. EEG changes following scopolamine administration in healthy subjects. Quantitative analysis during rest and photic stimulation. Neuropsychobiology 1999; 39: 219-26.

Klunk WE, Engler H, Nordberg A, Wang Y, Blomqvist G, Holt DP, et al. Imaging brain amyloid in Alzheimer's disease with Pittsburgh Compound-B. Ann Neurol 2004; 55: 306-19. 
Knopman DS, DeKosky ST, Cummings JL, Chui H, Corey-Bloom J, Relkin N, et al. Practice parameter: diagnosis of dementia (an evidence-based review). Report of the Quality Standards Subcommittee of the American Academy of Neurology. Neurology 2001; 56: 1143-53.

Koenig T, Prichep L, Dierks T, Hubl D, Wahlund LO, John ER, et al. Decreased EEG synchronization in Alzheimer's disease and mild cognitive impairment. Neurobiol Aging 2005; 26: 165-71.

Kogan EA, Korczyn AD, Virchovsky RG, Klimovizky S, Treves TA, Neufeld MY. EEG changes during long-term treatment with donepezil in Alzheimer's disease patients. J Neural Transm 2001; 108: 1167-73.

Kowalski JW, Gawel M, Pfeffer A, Barcikowska M. The diagnostic value of EEG in Alzheimer disease: correlation with the severity of mental impairment. J Clin Neurophysiol 2001; 18: 570-5.

Lanctot KL, Herrmann N, Mazzotta P, Khan LR, Ingber N. GABAergic function in Alzheimer's disease: evidence for dysfunction and potential as a therapeutic target for the treatment of behavioural and psychological symptoms of dementia. Can J Psychiatry 2004; 49: 439-53.

Lanctot KL, Moosa S, Herrmann N, Leibovitch FS, Rothenburg L, Cotter A, et al. A SPECT study of apathy in Alzheimer's disease. Dement Geriatr Cogn Disord 2007; 24: 65-72.

Landau AN, Esterman M, Robertson LC, Bentin S, Prinzmetal W. Different effects of voluntary and involuntary attention on EEG activity in the gamma band. J Neurosci 2007; 27: 11986-90.

Landes AM, Sperry SD, Strauss ME, Geldmacher DS. Apathy in Alzheimer's disease. J Am Geriatr Soc 2001; 49: 1700-7.

Larrieu S, Letenneur L, Orgogozo JM, Fabrigoule C, Amieva H, Le Carret N, et al. Incidence and outcome of mild cognitive impairment in a population-based prospective cohort. Neurology 2002; 59: 1594-9.

Lawrence AD, Evans AH, Lees AJ. Compulsive use of dopamine replacement therapy in Parkinson's disease: reward systems gone awry? Lancet Neurol 2003; 2: 595-604.

Levinoff EJ, Saumier D, Chertkow H. Focused attention deficits in patients with Alzheimer's disease and mild cognitive impairment. Brain Cogn 2005; 57: 127-30.

Levy R, Czernecki V. Apathy and the basal ganglia. J Neurol 2006; 253 Suppl 7: VII54-61. Levy $R$, Dubois B. Apathy and the functional anatomy of the prefrontal cortex-basal ganglia circuits. Cereb Cortex 2006; 16: 916-28.

Levy-Cooperman N, Burhan AM, Rafi-Tari S, Kusano M, Ramirez J, Caldwell C, et al. Frontal lobe hypoperfusion and depressive symptoms in Alzheimer disease. J Psychiatry Neurosci 2008; 33: 218-26. 
Lezak. Neuropsychological Assessment. New York Oxford: Oxford University Press, 1995a.

Lezak M. Neuropsychological Assessment. New York: NY: Oxford University Press, 1995b.

Locatelli T, Cursi M, Liberati D, Franceschi M, Comi G. EEG coherence in Alzheimer's disease. Electroencephalogr Clin Neurophysiol 1998; 106: 229-37.

Lopez OL, Smith G, Becker JT, Meltzer CC, DeKosky ST. The psychotic phenomenon in probable Alzheimer's disease: a positron emission tomography study. J Neuropsychiatry Clin Neurosci 2001a; 13: 50-5.

Lopez OL, Zivkovic S, Smith G, Becker JT, Meltzer CC, DeKosky ST. Psychiatric symptoms associated with cortical-subcortical dysfunction in Alzheimer's disease. J Neuropsychiatry Clin Neurosci 2001b; 13: 56-60.

Lowe SL, Francis PT, Procter AW, Palmer AM, Davison AN, Bowen DM. Gamma-aminobutyric acid concentration in brain tissue at two stages of Alzheimer's disease. Brain 1988; 111 ( Pt 4): 785-99.

Lustig C, Snyder AZ, Bhakta M, O'Brien KC, McAvoy M, Raichle ME, et al. Functional deactivations: change with age and dementia of the Alzheimer type. Proc Natl Acad Sci U S A 2003; 100: 14504-9.

Lyketsos CG, Lopez O, Jones B, Fitzpatrick AL, Breitner J, DeKosky S. Prevalence of neuropsychiatric symptoms in dementia and mild cognitive impairment: results from the cardiovascular health study. Jama 2002; 288: 1475-83.

Lyketsos CG, Sheppard JM, Steinberg M, Tschanz JA, Norton MC, Steffens DC, et al. Neuropsychiatric disturbance in Alzheimer's disease clusters into three groups: the Cache County study. Int J Geriatr Psychiatry 2001; 16: 1043-53.

Marin RS. Apathy: a neuropsychiatric syndrome. J Neuropsychiatry Clin Neurosci 1991; 3: 243-54.

Marin RS, Biedrzycki RC, Firinciogullari S. Reliability and validity of the Apathy Evaluation Scale. Psychiatry Res 1991; 38: 143-62.

Marshall GA, Fairbanks LA, Tekin S, Vinters HV, Cummings JL. Neuropathologic correlates of apathy in Alzheimer's disease. Dement Geriatr Cogn Disord 2006; 21: 144-7.

Marshall GA, Monserratt L, Harwood D, Mandelkern M, Cummings JL, Sultzer DL. Positron emission tomography metabolic correlates of apathy in Alzheimer disease. Arch Neurol 2007; 64: 1015-20.

Masdeu JC, Zubieta JL, Arbizu J. Neuroimaging as a marker of the onset and progression of Alzheimer's disease. J Neurol Sci 2005; 236: 55-64. 
McKhann G, Drachman D, Folstein M, Katzman R, Price D, Stadlan EM. Clinical diagnosis of Alzheimer's disease: report of the NINCDS-ADRDA Work Group under the auspices of Department of Health and Human Services Task Force on Alzheimer's Disease. Neurology 1984; 34: 939-44.

Mendez MF, Cherrier MM, Perryman KM. Differences between Alzheimer's disease and vascular dementia on information processing measures. Brain Cogn 1997; 34: 301-10.

Migneco O, Benoit M, Koulibaly PM, Dygai I, Bertogliati C, Desvignes P, et al. Perfusion brain SPECT and statistical parametric mapping analysis indicate that apathy is a cingulate syndrome: a study in Alzheimer's disease and nondemented patients. Neuroimage 2001; 13: 896-902.

Morris JC. The Clinical Dementia Rating (CDR): current version and scoring rules. Neurology 1993; 43: 2412-4.

Mosconi L, Tsui WH, Herholz K, Pupi A, Drzezga A, Lucignani G, et al. Multicenter standardized 18F-FDG PET diagnosis of mild cognitive impairment, Alzheimer's disease, and other dementias. J Nucl Med 2008; 49: 390-8.

Moss MB, Albert MS, Butters N, Payne M. Differential patterns of memory loss among patients with Alzheimer's disease, Huntington's disease, and alcoholic Korsakoff's syndrome. Arch Neurol 1986; 43: 239-46.

Naatanen R, Paavilainen P, Alho K, Reinikainen K, Sams M. The mismatch negativity to intensity changes in an auditory stimulus sequence. Electroencephalogr Clin Neurophysiol Suppl 1987; 40: 125-31.

Nakano S, Yamashita F, Matsuda H, Kodama C, Yamada T. Relationship between delusions and regional cerebral blood flow in Alzheimer's disease. Dement Geriatr Cogn Disord 2006; 21: 16-21.

Nardone R, Bratti A, Tezzon F. Motor cortex inhibitory circuits in dementia with Lewy bodies and in Alzheimer's disease. J Neural Transm 2006a; 113: 1679-84.

Nardone R, Marth R, Ausserer H, Bratti A, Tezzon F. Reduced short latency afferent inhibition in patients with Down syndrome and Alzheimer-type dementia. Clin Neurophysiol 2006b; 117: 2204-10.

Neshige R, Barrett G, Shibasaki H. Auditory long latency event-related potentials in Alzheimer's disease and multi-infarct dementia. J Neurol Neurosurg Psychiatry 1988; 51: 1120-5.

Nestor PJ, Scheltens P, Hodges JR. Advances in the early detection of Alzheimer's disease. Nat Med 2004; 10 Suppl: S34-41. 
Neuropathology Group of the Medical Research Council Cognitive Function and Ageing Study (MRC CFAS). Pathological correlates of late-onset dementia in a multicentre, community-based population in England and Wales. Neuropathology Group of the Medical Research Council Cognitive Function and Ageing Study (MRC CFAS). Lancet 2001; 357: 169-75.

O'Conner KP. Slow potential correlates of attention dysfunction in senile dementia: I. Biol Psychol 1980; 11: 193-202.

Onofrj M, Thomas A, Luciano AL, lacono D, Di Rollo A, D'Andreamatteo G, et al. Donepezil versus vitamin $\mathrm{E}$ in Alzheimer's disease: Part 2: mild versus moderatesevere Alzheimer's disease. Clin Neuropharmacol 2002; 25: 207-15.

Osipova D, Pekkonen E, Ahveninen J. Enhanced magnetic auditory steady-state response in early Alzheimer's disease. Clin Neurophysiol 2006; 117: 1990-5.

Ott BR, Tate CA, Gordon NM, Heindel WC. Gender differences in the behavioral manifestations of Alzheimer's disease. J Am Geriatr Soc 1996; 44: 583-7.

Patterson JV, Michalewski HJ, Starr A. Latency variability of the components of auditory event-related potentials to infrequent stimuli in aging, Alzheimer-type dementia, and depression. Electroencephalogr Clin Neurophysiol 1988; 71: 450-60.

Pekkonen E, Hirvonen J, Jaaskelainen IP, Kaakkola S, Huttunen J. Auditory sensory memory and the cholinergic system: implications for Alzheimer's disease. Neuroimage 2001; 14: 376-82.

Pekkonen E, Jaaskelainen IP, Kaakkola S, Ahveninen J. Cholinergic modulation of preattentive auditory processing in aging. Neuroimage 2005; 27: 387-92.

Pereda E, Quiroga RQ, Bhattacharya J. Nonlinear multivariate analysis of neurophysiological signals. Prog Neurobiol 2005; 77: 1-37.

Perry RJ, Hodges JR. Attention and executive deficits in Alzheimer's disease. A critical review. Brain 1999; 122 ( Pt 3): 383-404.

Petersen RC. Mild cognitive impairment as a diagnostic entity. J Intern Med 2004; 256 : 183-94.

Petersen RC, Doody R, Kurz A, Mohs RC, Morris JC, Rabins PV, et al. Current concepts in mild cognitive impairment. Arch Neurol 2001; 58: 1985-92.

Petersen RC, Smith GE, Waring SC, Ivnik RJ, Tangalos EG, Kokmen E. Mild cognitive impairment: clinical characterization and outcome. Arch Neurol 1999; 56: 303-8.

Phan KL, Wager T, Taylor SF, Liberzon I. Functional neuroanatomy of emotion: a metaanalysis of emotion activation studies in PET and fMRI. Neuroimage 2002; 16 : 331-48.

Pokryszko-Dragan A, Slotwinski K, Podemski R. Modality-specific changes in P300 parameters in patients with dementia of the Alzheimer type. Med Sci Monit 2003; 9: CR130-4. 
Polich J. Updating P300: an integrative theory of P3a and P3b. Clin Neurophysiol 2007; 118: 2128-48.

Polich J, Corey-Bloom J. Alzheimer's disease and P300: review and evaluation of task and modality. Curr Alzheimer Res 2005; 2: 515-25.

Polich J, Herbst KL. P300 as a clinical assay: rationale, evaluation, and findings. Int J Psychophysiol 2000; 38: 3-19.

Polich J, Ladish C, Bloom FE. P300 assessment of early Alzheimer's disease. Electroencephalogr Clin Neurophysiol 1990; 77: 179-89.

Polich J, Pitzer A. P300 and Alzheimer's disease: oddball task difficulty and modality effects. Electroencephalogr Clin Neurophysiol Suppl 1999; 50: 281-7.

Pruessner JC, Li LM, Serles W, Pruessner M, Collins DL, Kabani N, et al. Volumetry of hippocampus and amygdala with high-resolution MRI and three-dimensional analysis software: minimizing the discrepancies between laboratories. Cereb Cortex 2000; 10: 433-42.

Prvulovic D, Van de Ven V, Sack AT, Maurer K, Linden DE. Functional activation imaging in aging and dementia. Psychiatry Res 2005; 140: 97-113.

Reisberg B, Ferris SH, de Leon MJ, Crook T. The Global Deterioration Scale for assessment of primary degenerative dementia. Am J Psychiatry 1982; 139: 1136-9.

Reisberg B, Ferris, S. H., de Leon, M. J., Crook, T. The Global Deterioration Scale for assessment of primary degenerative dementia. American Journal of Psychiatry 1982; 139: $1136-9$.

Ribary U, loannides AA, Singh KD, Hasson R, Bolton JP, Lado F, et al. Magnetic field tomography of coherent thalamocortical $40-\mathrm{Hz}$ oscillations in humans. Proc Natl Acad Sci U S A 1991; 88: 11037-41.

Robert PH, Berr C, Volteau M, Bertogliati C, Benoit M, Sarazin M, et al. Apathy in patients with mild cognitive impairment and the risk of developing dementia of Alzheimer's disease: a one-year follow-up study. Clin Neurol Neurosurg 2006a; 108: 733-6.

Robert PH, Clairet S, Benoit M, Koutaich J, Bertogliati C, Tible O, et al. The apathy inventory: assessment of apathy and awareness in Alzheimer's disease, Parkinson's disease and mild cognitive impairment. Int J Geriatr Psychiatry 2002; 17: 1099-105.

Robert PH, Darcourt G, Koulibaly MP, Clairet S, Benoit M, Garcia R, et al. Lack of initiative and interest in Alzheimer's disease: a single photon emission computed tomography study. Eur J Neurol 2006b; 13: 729-35.

Rodriguez G, Copello F, Vitali P, Perego G, Nobili F. EEG spectral profile to stage Alzheimer's disease. Clin Neurophysiol 1999; 110: 1831-7.

Rodriguez G, Vitali P, De Leo C, De Carli F, Girtler N, Nobili F. Quantitative EEG changes in Alzheimer patients during long-term donepezil therapy. Neuropsychobiology 2002; 46: 49-56. 
Rodriguez R, Kallenbach U, Singer W, Munk MH. Short- and long-term effects of cholinergic modulation on gamma oscillations and response synchronization in the visual cortex. J Neurosci 2004; 24: 10369-78.

Rosen HJ, Allison SC, Schauer GF, Gorno-Tempini ML, Weiner MW, Miller BL. Neuroanatomical correlates of behavioural disorders in dementia. Brain 2005; 128: 2612-25.

Rosen WG, Mohs RC, Davis KL. A new rating scale for Alzheimer's disease. Am J Psychiatry 1984; 141: 1356-64.

Rosen WG, Terry RD, Fuld PA, Katzman R, Peck A. Pathological verification of ischemic score in differentiation of dementias. Ann Neurol 1980; 7: 486-8.

Roth M, Tym E, Mountjoy CQ, Huppert FA, Hendrie H, Verma S, et al. CAMDEX. A standardised instrument for the diagnosis of mental disorder in the elderly with special reference to the early detection of dementia. Br J Psychiatry 1986; 149: 698-709.

Salthouse TA. The processing-speed theory of adult age differences in cognition. Psychol Rev 1996; 103: 403-28.

Salthouse TA. Aging and measures of processing speed. Biol Psychol 2000; 54: 35-54.

Sanders A. Elements of Human Performance. Energetics, Stress and Sustained Attention. New Jersey: Lawrence Erlbaum Associates, 1998: 394-451.

Scheltens P, Launer LJ, Barkhof F, Weinstein HC, Jonker C. The diagnostic value of magnetic resonance imaging and technetium 99m-HMPAO single-photon-emission computed tomography for the diagnosis of Alzheimer disease in a communitydwelling elderly population. Alzheimer Dis Assoc Disord 1997; 11: 63-70.

Schmand B, Bakker D, Saan R, Louman J. [The Dutch Reading Test for Adults: a measure of premorbid intelligence level]. Tijdschr Gerontol Geriatr 1991; 22: 15-9.

Schmand B, Geerlings MI, Jonker C, Lindeboom J. Reading ability as an estimator of premorbid intelligence: does it remain stable in emergent dementia? J Clin Exp Neuropsychol 1998; 20: 42-51.

Schnitzler A, Gross J. Normal and pathological oscillatory communication in the brain. Nat Rev Neurosci 2005; 6: 285-96.

Shin IS, Carter M, Masterman D, Fairbanks L, Cummings JL. Neuropsychiatric symptoms and quality of life in Alzheimer disease. Am J Geriatr Psychiatry 2005; 13: 469-74.

Shoghi-Jadid K, Small GW, Agdeppa ED, Kepe V, Ercoli LM, Siddarth P, et al. Localization of neurofibrillary tangles and beta-amyloid plaques in the brains of living patients with Alzheimer disease. Am J Geriatr Psychiatry 2002; 10: 24-35.

Simpson PMS, D. J. Wesnes, K. A. Wilcock, G. K. The cognitive drug research computerized assessment system for demented patients: A validation study. International Journal of Geriatric Psychiatry 1991; 6: 95-102.

Singer W, Gray CM. Visual feature integration and the temporal correlation hypothesis. Annu Rev Neurosci 1995; 18: 555-86. 
Sjogren M, Vanderstichele $H$, Agren $H$, Zachrisson O, Edsbagge M, Wikkelso C, et al. Tau and Abeta42 in cerebrospinal fluid from healthy adults 21-93 years of age: establishment of reference values. Clin Chem 2001; 47: 1776-81.

Sneddon R, Shankle WR, Hara J, Rodriquez A, Hoffman D, Saha U. QEEG monitoring of Alzheimer's disease treatment: a preliminary report of three case studies. Clin EEG Neurosci 2006; 37: 54-9.

Staff RT, Shanks MF, Macintosh L, Pestell SJ, Gemmell HG, Venneri A. Delusions in Alzheimer's disease: spet evidence of right hemispheric dysfunction. Cortex 1999; 35: 549-60.

Stam CJ, Jones BF, Manshanden I, van Cappellen van Walsum AM, Montez T, Verbunt $J P$, et al. Magnetoencephalographic evaluation of resting-state functional connectivity in Alzheimer's disease. Neuroimage 2006; 32: 1335-44.

Stam CJ, van Cappellen van Walsum AM, Pijnenburg YA, Berendse HW, de Munck JC, Scheltens $P$, et al. Generalized synchronization of MEG recordings in Alzheimer's Disease: evidence for involvement of the gamma band. J Clin Neurophysiol 2002; 19: 562-74.

Stam CJ, van der Made Y, Pijnenburg YA, Scheltens P. EEG synchronization in mild cognitive impairment and Alzheimer's disease. Acta Neurol Scand 2003; 108: 90-6.

Steinberg M, Corcoran C, Tschanz JT, Huber C, Welsh-Bohmer K, Norton MC, et al. Risk factors for neuropsychiatric symptoms in dementia: the Cache County Study. Int J Geriatr Psychiatry 2006; 21: 824-30.

Steinberg M, Shao H, Zandi P, Lyketsos CG, Welsh-Bohmer KA, Norton MC, et al. Point and 5-year period prevalence of neuropsychiatric symptoms in dementia: the Cache County Study. Int J Geriatr Psychiatry 2008; 23: 170-7.

Steinberg M, Sheppard JM, Tschanz JT, Norton MC, Steffens DC, Breitner JC, et al. The incidence of mental and behavioral disturbances in dementia: the cache county study. J Neuropsychiatry Clin Neurosci 2003; 15: 340-5.

Steinberg M, Tschanz JT, Corcoran C, Steffens DC, Norton MC, Lyketsos CG, et al. The persistence of neuropsychiatric symptoms in dementia: the Cache County Study. Int J Geriatr Psychiatry 2004; 19: 19-26.

Stopfer M, Bhagavan S, Smith BH, Laurent G. Impaired odour discrimination on desynchronization of odour-encoding neural assemblies. Nature 1997; 390: 70-4.

Storandt M, Beaudreau S. Do reaction time measures enhance diagnosis of early-stage dementia of the Alzheimer type. Arch Clin Neuropsychol 2004; 19: 119-24.

Storandt M, Hill RD. Very mild senile dementia of the Alzheimer type. II. Psychometric test performance. Arch Neurol 1989; 46: 383-6.

Strozyk D, Blennow K, White LR, Launer LJ. CSF Abeta 42 levels correlate with amyloidneuropathology in a population-based autopsy study. Neurology 2003; 60: 652-6. 
Szelies B, Mielke R, Grond M, Heiss WD. P300 in Alzheimer's disease: relationships to dementia severity and glucose metabolism. J Neurol Sci 1995; 130: 77-81.

Tallon-Baudry C, Bertrand O. Oscillatory gamma activity in humans and its role in object representation. Trends Cogn Sci 1999; 3: 151-162.

Tallon-Baudry C, Bertrand O, Henaff MA, Isnard J, Fischer C. Attention modulates gamma-band oscillations differently in the human lateral occipital cortex and fusiform gyrus. Cereb Cortex 2005; 15: 654-62.

Tallon-Baudry C, Bertrand O, Peronnet F, Pernier J. Induced gamma-band activity during the delay of a visual short-term memory task in humans. J Neurosci 1998; 18: 4244-54.

Tallon-Baudry C, Mandon S, Freiwald WA, Kreiter AK. Oscillatory synchrony in the monkey temporal lobe correlates with performance in a visual short-term memory task. Cereb Cortex 2004; 14: 713-20.

Tekin S, Cummings JL. Frontal-subcortical neuronal circuits and clinical neuropsychiatry: an update. J Psychosom Res 2002; 53: 647-54.

Tekin S, Mega MS, Masterman DM, Chow T, Garakian J, Vinters HV, et al. Orbitofrontal and anterior cingulate cortex neurofibrillary tangle burden is associated with agitation in Alzheimer disease. Ann Neurol 2001; 49: 355-61.

The Ronald and Nancy Reagan Research Institute of the Alzheimer's Association and the National Institute on Aging Working Group. Consensus report of the Working Group on: "Molecular and Biochemical Markers of Alzheimer's Disease". The Ronald and Nancy Reagan Research Institute of the Alzheimer's Association and the National Institute on Aging Working Group. Neurobiol Aging 1998; 19: 109-16.

Thomas A, lacono D, Bonanni L, D'Andreamatteo G, Onofrj M. Donepezil, rivastigmine, and vitamin E in Alzheimer disease: a combined P300 event-related potentials/ neuropsychologic evaluation over 6 months. Clin Neuropharmacol 2001; 24: 31-42.

Tiitinen H, Sinkkonen J, Reinikainen K, Alho K, Lavikainen J, Naatanen R. Selective attention enhances the auditory $40-\mathrm{Hz}$ transient response in humans. Nature 1993; 364: 59-60.

Traub RD, Whittington MA, Stanford IM, Jefferys JG. A mechanism for generation of long-range synchronous fast oscillations in the cortex. Nature 1996; 383: 621-4.

Tun SM, Murman DL, Long HL, Colenda CC, von Eye A. Predictive validity of neuropsychiatric subgroups on nursing home placement and survival in patients with Alzheimer disease. Am J Geriatr Psychiatry 2007; 15: 314-27.

van Boxtel MP, Buntinx F, Houx PJ, Metsemakers JF, Knottnerus A, Jolles J. The relation between morbidity and cognitive performance in a normal aging population. J Gerontol A Biol Sci Med Sci 1998; 53: M147-54. 
Van der Lubbe RH, Los SA, Jaskowski P, Verleger R. Being prepared on time: on the importance of the previous foreperiod to current preparation, as reflected in speed, force and preparation-related brain potentials. Acta Psychol (Amst) 2004; 116: 245-62. van Deursen JA, Vuurman EF, Verhey FR, van Kranen-Mastenbroek VH, Riedel WJ. Increased EEG gamma band activity in Alzheimer's disease and mild cognitive impairment. J Neural Transm 2008; 115: 1301-11.

van Deursen J.A., Vuurman E.F.P.M., van Kranen-Mastenbroek V.H., Verhey F.R.J., Riedel W.J. (2009). 40-Hz steady state response in Alzheimer's disease and mild cognitive impairment. Neurobiology of Aging, In press.

van Harten B, Laman DM, van Duijn H, Knol DL, Stam CJ, Scheltens P, et al. The auditory oddball paradigm in patients with vascular cognitive impairment: a prolonged latency of the N2 complex. Dement Geriatr Cogn Disord 2006; 21: 322-7.

Verhaeghen P, De Meersman L. Aging and the Stroop effect: a meta-analysis. Psychol Aging 1998; 13: 120-6.

Verhey FR, Houx P, Van Lang N, Huppert F, Stoppe G, Saerens J, et al. Cross-national comparison and validation of the Alzheimer's Disease Assessment Scale: results from the European Harmonization Project for Instruments in Dementia (EURO-HARPID). Int J Geriatr Psychiatry 2004; 19: 41-50.

Verhey FR, Jolles J, Ponds RW, Rozendaal N, Plugge LA, de Vet RC, et al. Diagnosing dementia: a comparison between a monodisciplinary and a multidisciplinary approach. J Neuropsychiatry Clin Neurosci 1993; 5: 78-85.

Verleger R, Kompf D, Neukater W. Event-related EEG potentials in mild dementia of the Alzheimer type. Electroencephalogr Clin Neurophysiol 1992; 84: 332-43.

Vink $M$, Jolles J. A new version of the Trail Making Test as an information processing task. J Clin Neuropsychol 1985a; 7: 162.

Vink M, Jolles J. A new version of the Trial Making Test as an information processing task. J Clin Neuropsychol 1985b: 162.

Visser PJ, Brodaty H. MCl is not a clinically useful concept. Int Psychogeriatr 2006; 18 : 402-9; discussion 409-14.

Visser PJ, Scheltens P, Pelgrim E, Verhey FR. Medial temporal lobe atrophy and APOE genotype do not predict cognitive improvement upon treatment with rivastigmine in Alzheimer's disease patients. Dement Geriatr Cogn Disord 2005; 19: 126-33.

Visser PJ, Verhey FR, Hofman PA, Scheltens P, Jolles J. Medial temporal lobe atrophy predicts Alzheimer's disease in patients with minor cognitive impairment. J Neurol Neurosurg Psychiatry 2002; 72: 491-7.

Walter WG, Cooper R, Aldridge VJ, McCallum WC, Winter AL. Contingent Negative Variation: an Electric Sign of Sensorimotor Association and Expectancy in the Human Brain. Nature 1964; 203: 380-4. 
Wang L, Swank JS, Glick IE, Gado MH, Miller MI, Morris JC, et al. Changes in hippocampal volume and shape across time distinguish dementia of the Alzheimer type from healthy aging. Neuroimage 2003; 20: 667-82.

Welsh K, Butters N, Hughes J, Mohs R, Heyman A. Detection of abnormal memory decline in mild cases of Alzheimer's disease using CERAD neuropsychological measures. Arch Neurol 1991; 48: 278-81.

Welsh KA, Butters N, Hughes JP, Mohs RC, Heyman A. Detection and staging of dementia in Alzheimer's disease. Use of the neuropsychological measures developed for the Consortium to Establish a Registry for Alzheimer's Disease. Arch Neurol 1992; 49: 448-52.

Werber AE, Klein C, Rabey JM. Evaluation of cholinergic treatment in demented patients by P300 evoked related potentials. Neurol Neurochir Pol 2001; 35 Suppl 3: 37-43.

Werber EA, Gandelman-Marton R, Klein C, Rabey JM. The clinical use of P300 event related potentials for the evaluation of cholinesterase inhibitors treatment in demented patients. J Neural Transm 2003; 110: 659-69.

Wesnes KA. Assessing change in cognitive function in dementia: the relative utilities of the Alzheimer's Disease Assessment Scale-Cognitive Subscale and the Cognitive Drug Research system. Neurodegener Dis 2008; 5: 261-3.

Whitham EM, Pope KJ, Fitzgibbon SP, Lewis T, Clark CR, Loveless S, et al. Scalp electrical recording during paralysis: quantitative evidence that EEG frequencies above $20 \mathrm{~Hz}$ are contaminated by EMG. Clin Neurophysiol 2007; 118: 1877-88.

Whittington MA, Faulkner HJ, Doheny HC, Traub RD. Neuronal fast oscillations as a target site for psychoactive drugs. Pharmacol Ther 2000; 86: 171-90.

Whittington MA, Traub RD, Jefferys JG. Synchronized oscillations in interneuron networks driven by metabotropic glutamate receptor activation. Nature 1995; 373: 612-5.

Whitwell JL, Josephs KA, Murray ME, Kantarci K, Przybelski SA, Weigand SD, et al. MRI correlates of neurofibrillary tangle pathology at autopsy: a voxel-based morphometry study. Neurology 2008; 71: 743-9.

Williams LM, Simms E, Clark CR, Paul RH, Rowe D, Gordon E. The test-retest reliability of a standardized neurocognitive and neurophysiological test battery: "neuromarker". Int J Neurosci 2005; 115: 1605-30.

Williams PA, Jones GH, Briscoe M, Thomas R, Cronin P. P300 and reaction-time measures in senile dementia of the Alzheimer type. Br J Psychiatry 1991; 159: 410-4.

Wimo A, Winblad B, Aguero-Torres $H$, von Strauss E. The magnitude of dementia occurrence in the world. Alzheimer Dis Assoc Disord 2003; 17: 63-7.

Woods RP. Characterizing volume and surface deformations in an atlas framework: theory, applications, and implementation. Neuroimage 2003; 18: 769-88. 
Wright $\mathrm{Cl}$, Dickerson BC, Feczko E, Negeira A, Williams D. A functional magnetic resonance imaging study of amygdala responses to human faces in aging and mild Alzheimer's disease. Biol Psychiatry 2007; 62: 1388-95.

Ylikoski R, Jokinen H, Andersen P, Salonen O, Madureira S, Ferro J, et al. Comparison of the Alzheimer's Disease Assessment Scale Cognitive Subscale and the Vascular Dementia Assessment Scale in differentiating elderly individuals with different degrees of white matter changes. The LADIS Study. Dement Geriatr Cogn Disord 2007; 24: 73-81.

Zappoli R, Arnetoli G, Paganini M, Versari A, Battaglia A, Grignani A, et al. Contingent negative variation and reaction time in patients with presenile idiopathic cognitive decline and presenile Alzheimer-type dementia. Preliminary report on long-term nicergoline treatment. Neuropsychobiology 1987; 18: 149-54.

Zappoli R, Versari A, Arnetoli G, Paganini M, Muscas GC, Arneodo MG, et al. Topographic CNV activity mapping, presenile mild primary cognitive decline and Alzheimertype dementia. Neurophysiol Clin 1991; 21: 473-83.

Zemlan FP, Rosenberg WS, Luebbe PA, Campbell TA, Dean GE, Weiner NE, et al. Quantification of axonal damage in traumatic brain injury: affinity purification and characterization of cerebrospinal fluid tau proteins. J Neurochem 1999; 72: 741-50. 


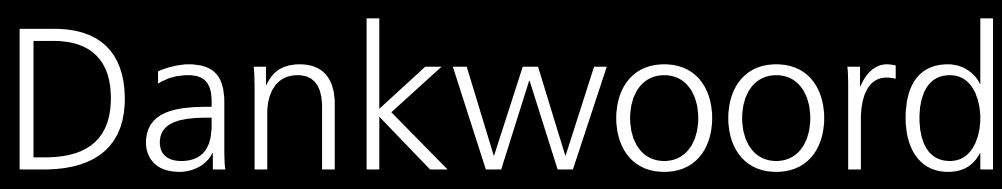



$\mathrm{Nu}$ het wetenschappelijk werk erop zit is het tijd om het waarschijnlijk meest populaire hoofdstuk van dit proefschrift te schrijven. Ondanks het feit dat het schrijven van een proefschrift soms een eenzame missie is, zou dit proefschrift niet tot stand zijn gekomen zonder de intellectuele, praktische en emotionele steun van een heel aantal mensen.

Ten eerste de patiënten, proefpersonen en hun naasten; zonder hun deelname was dit klinisch wetenschappelijk onderzoek onmogelijk geweest. Ik wil u danken voor de tijd en het vertrouwen dat u gesteld heeft in dit onderzoek.

Mijn eerste promotor Prof. Wim Riedel: beste Wim, ik wil je danken voor de vele en gedetailleerde commentaren die je leverde op mijn manuscripten. Onze vele telefonische overleggen over manuscripten, artikelen en al wat met onderzoek te maken heeft is een enorme leerschool gebleken. Je nauwgezette werkwijze en theoretische kennis heeft me vaak verbaasd.

Mijn tweede promotor Prof. Frans Verhey: beste Frans, je hebt me geleerd om mijn soms warrige ideeën en teksten te structuren tot een coherent geheel. Ik wil je danken voor het vertrouwen dat je in me had en voor de vrijheid die je me hebt gegeven om me te ontwikkelen tot een zelfstandig wetenschapper. Mijn co-promotor Dr. Eric Vuurman: beste Eric, jouw manier van begeleiden laat zich het best kenmerken door jouw coachend vermogen en enthousiasme over het onderzoek. Jouw ontspannen en vriendschappelijke manier van begeleiden heeft me vaak gemotiveerd en is van enorme waarde geweest in dit project! Ik ben nog steeds dankbaar voor het feit dat ik een jaar of zes geleden bij jou binnenstapte voor een stageplaats.

Co-auteur van twee van voorafgaande hoofdstukken, Dr. Pauline Aalten. Pauline ik wil je danken voor onze voortreffelijke samenwerking tijdens het VBM onderzoek. Jouw kennis van neuropsychiatrie en mijn meer technische achtergrond vulden elkaar prima aan. Daarnaast waren onze discussies over het nut van sneeuw een aangename bijkomstigheid.

Co-auteur op drie van de EEG hoofdstukken, Dr. Vivianne van Kranen-Mastenbroek. Beste Vivianne, ik wil je danken voor je technische en wetenschappelijke bijdrage aan de EEG analysen. Jouw adviezen over het efficiënt oplossen van een aantal technische EEG problemen hebben het onderzoek enorm vooruit geholpen.

Daarnaast wil ik Dr. Paul Hofman danken voor zijn nuttige aanvullingen bij het schrijven van de laatste hoofdstukken. Beste Paul, mijn interesse voor wetenschappelijk onderzoek werd gewekt toen ik een heel aantal jaar geleden mijn 
afstudeerstage van de MBRT bij je deed. Ik heb er sindsdien nooit spijt van gehad dat ik de wetenschap in gegaan ben.

Beste Rosa, ik wil je danken voor de vele NPO's die je hebt afgenomen tijdens de huisbezoeken aan de patiënten. Deze bezoeken werden met name opgeleukt door jouw ongelimiteerde goede zin en gezellige gebabbel.

Dan de twee stagiaires die een deel van de data acquisitie en data verwerking hebben gedaan, Lieke Smits en Laura Schellekens, veel dank voor jullie assistentie en enthousiasme.

Voor de assistentie in computertechnische zin ben ik Marc Geerlings, Nico Rozendaal en Ron Mengelers erg dankbaar. Beste Marc, zonder jouw scriptjes was ik nu nog MRI data aan het analyseren. Ron en Nico, veel dank voor het oplossen van alle problemen die voor kunnen komen wanneer de werelden van Macintosh en Microsoft elkaar ontmoeten.

Zonder een goede werksfeer wordt elk promotie traject een zware opgave. Als eerst wil mijn kamergenoot Elsbeth danken voor haar gezelligheid maar met name voor haar geduld. Immers, het delen van een kamer met twee aio's die hun proefschrift aan het afronden zijn was vast niet altijd een pretje. Voor alle gezelligheid, aio-uitjes en vaak levendige discussies ben ik Saartje, Floortje, Ed, Jennifer, Inez, Heidi, Lia, Rosa's, Claire, Lyzel, Helen, Richel, Geert, Sebastian, Margriet, Esthers, Nikkie, Sanne, Ellissa, Martin, Caroline, Olga, Jelle, Aukje, Renate, Astrid, Elsa, Inez, Nettie, Laurant, Marco, Ankie, Els, Wim, Lisbeth, Annemarie, Bart, Dymphie, Celeste, Deliane, (Tequila) Tim, Kim, Eef, Katja, Anita, Marleen, Jan, Arjan, Pascal, Annemiek, Petra, Peter, Silke en Anke heel erg dankbaar.

Jacky Rinket en Tess Vuurman, ik wil jullie enorm bedanken voor jullie creativiteit en snelheid bij het verzorgen van de layout en het ontwerpen van de omslag. Het is erg mooi geworden!

Vanzelfsprekend ben ik de paranimfen Sharon Bouwens en Peter van Ruitenbeek erg dankbaar voor jullie steun en vriendschap. Ik ben ontzettend blij dat jullie achter mij staan op deze promotie. Beste Sharon, wij zijn ongeveer gelijktijdig begonnen met ons promotietraject en wij werden dan ook samen in een kamertje gezet. Dat bleek erg goed uit te pakken want onze vele discussies en met name de slappe 'typisch Eindhovense' humor (dekentonoit) zorgde voor veel plezier tijdens het werk. Ik vind het wel jammer dat het record agendatennis nooit boven de 19 is uitgekomen. Beste Peter, dankzij jou weet ik nu alles van 
de geschiedenis van BMW en een piepklein beetje meer over hoe een auto werkt. Jouw enthousiasme over wetenschap is aanstekelijk. Ik ben je ook erg dankvoor de gezellige avondjes tijdens de bezoeken aan congressen.

Tot bijna slot dank ik mijn vrienden en familie voor hun steun, de prettige afleiding bij het schrijven en hun interesse in mijn 'boekje'. In het bijzonder, Pap, Mam, Judith en Geofrey; veel dank voor jullie onvoorwaardelijke steun, liefde en vertrouwen. Pap en Mam, jullie hebben me geleerd altijd mijn hart te volgen en dat er zonder hard werken niets bereikt kan worden.

Het allerbeste komt als laatst. Lieve Maartje, ik ben je dankbaar voor eigenlijk alles. Het woord dank is een understatement. Zonder jouw steun, relativeringsvermogen, geduld, en onbeperkte liefde was dit proefschrift er waarschijnlijk nooit gekomen. 



$$
\begin{array}{r}
\text { Curriculum } \\
\text { vitae }
\end{array}
$$



Jeroen van Deursen werd op 17 december 1979 geboren te Helmond. Hij deed eindexamen HAVO aan het Dr. Knippenbergcollege in Helmond. De studie Medisch Beeldvormende en Radiotherapeutische Technieken werd afgerond in 2001. De studie Gezondheidswetenschappen met als afstudeerrichting Biologische Gezondheidskunde werd afgesloten in 2004. In januari 2005 begon hij met zijn promotieonderzoek aan de Maastricht School for Mental Health and Neuroscience. Daar heeft hij onderzoek verricht naar structurele en functionele hersen-veranderingen in de ziekte van Alzheimer onder leiding van Prof. dr. Wim Riedel, Prof. dr. Frans Verhey en dr. Eric Vuurman. Dit onderzoek heeft geresulteerd in het huidige proefschrift. Momenteel is hij werkzaam bij het Medtronic Bakken Research Center te Maastricht. 

Publications 

- Van der Veen F.M., Evers E.A.T., van Deursen J.A., Deutz N.E.P., Backes W.H., Schmitt J.A.J. (2006).

Acute tryptophan depletion reduces activation in the right hippocampus during encoding in an episodic memory task. Neuroimage 31(6) 1188-96

- Theunissen E.L., van Kroonenburg M.J., van Deursen J.A., Blom-Coenjaerts C., Ramaekers G. (2006).

Stimulating effects of the antihistamine fexofenadine: testing the dopamine transporter hypothesis. Psychopharmacology 187(1) 95-102

- Evers E.A., Van der Veen F.M., van Deursen J.A., Schmitt J.A., Deutz N.E., Jolles J. (2006).

The effect of acute tryptophan depletion on the BOLD response during performance monitoring and response inhibition in healthy male volunteers. Psychopharmacology $187(2) 200-8$

- Kempen G.I., Meier A.J., Bouwens S.F., van Deursen J.A., Verhey, F.R. (2007).

The psychometric properties of the Dutch version of the Telephone Interview Cognitive Status (TICS). (article in Dutch) Tijdschrift voor Gerontologie en Geriatrie 38(1) 38-45

- van Deursen J.A., Vuurman E.F., Verhey, F.R., van Kranen-Mastenbroek V.H., Riedel W.J. (2008).

Induced EEG gamma band power is increased in Alzheimer's Disease and MCl. Journal of Neural Transmission 115(9) 1301-11

- van Deursen J.A., Vuurman E.F.P.M., Smits L.L., Verhey F.R.J., Riedel W.J. (2009). Response Speed, Contingent Negative Variation and P300 in Alzheimer's Disease and $\mathrm{MCl}$. Brain and Cognition, in press

- van Deursen J.A., Vuurman E.F.P.M., van Kranen-Mastenbroek V.H., Verhey F.R.J., Riedel W.J. (2009).

40-Hz steady state response in Alzheimer's disease and mild cognitive impairment. Neurobiology of Aging, in press

- van Deursen J.A., Vuurman E.F.P.M., Verhey F.R.J., Riedel W.J. (2009). Comparison of EEG paradigms in the diagnosis of $A D$ and $M C l$. Submitted

- van Deursen J.A., Verhey F.R., Hofman P.A., Barkof F., Aalten P. (2009). Gray Matter Correlates of Neuropsychiatric Syndromes in Alzheimer's Disease. Submitted

- Aalten P., van Deursen J.A., Hofman P.A., Barkof F., Verhey F.R. (2009). Volumetric Correlates of Apathy in Alzheimer's Disease. Submitted

- van Deursen J.A., Schmitt J.A.J, Evers E.A.T, Backes W.H., Van der Veen F.M. Effects of acute tryptophan depletion on brain activity in an episodic memory task; an fMRI study. Third Dutch Endo-Neuro-Psycho Meeting, Doorwerth. June 2004. (poster) 
Functional and structural brain markers of Alzheimer's disease | Publications

- van Deursen J.A., Vuurman E.F., Riedel W.J., Verhey F.R. Induced EEG gamma band power is increased in Alzheimer's Disease and MCl. Conference for Applied Neuroscience of Healthy Brain Function, Nijmegen. May 2007. (oral presentation)

- van Deursen J.A., Vuurman E.F., Riedel W.J., Verhey F.R. Induced EEG gamma band power is increased in Alzheimer's Disease and MCI. Sixth Ditch Endo-Neuro-Psycho Meeting, Doorwerth. June 2007. (oral presentation) 